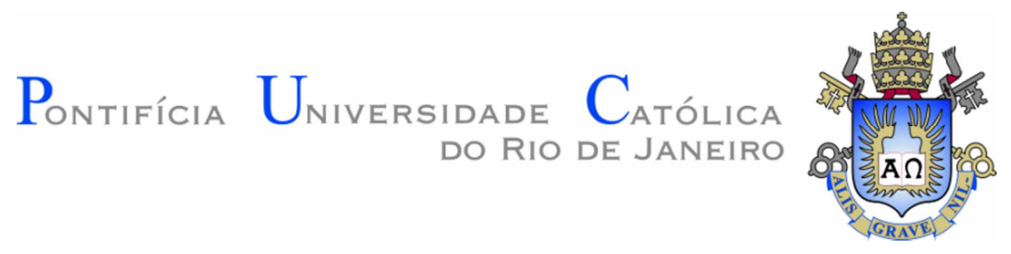

Manoel Feliciano da Silva Junior

\title{
Intelligent Well Transient Temperature \\ Signal Reconstruction
}

TESE DE DOUTORADO

Thesis presented to the Programa de Pos-Graduação em Engenharia Mecânica of the Departamento de Engenharia Mecânica, PUC-Rio as partial fulfillment of the requirements for the degree of Doutor em Engenharia Mecânica.

Advisor: Prof. Marcio da Silveira Carvalho Co-Advisor: Prof. Arthur Martins Barbosa Braga 


\title{
Manoel Feliciano da Silva Junior
}

\section{Intelligent Well Transient Temperature Signal \\ Reconstruction}

Thesis presented to the Programa de Pos-Grauduação em Engenharia Mecânica of the Departamento de Engenharia Mecânica do Centro Técnico Científico da PUC-Rio, as partial fulfillment of the requirements for the degree of Doutor.

\author{
Prof. Marcio da Silveira Carvalho \\ Advisor \\ Mechanical Engineering Department - PUC-Rio \\ Prof. Arthur Martins Barbosa Braga \\ Co-Advisor \\ Mechanical Engineering Department - PUC-Rio \\ Dr. Regis Kruel Romeu \\ Centro de Pesquisas e Desenvolvimento Leopoldo Miguez - Petrobras \\ Prof. Ubirajara Franco Moreno \\ Department of Automation and Systems Engineering- UFSC \\ Prof. Ram Rajagopal \\ Civil and Environmental Engineering - Stanford \\ Prof. Luis Fernando Alzuguir de Azevedo \\ Mechanical Engineering Department - PUC-Rio \\ Prof. José Eugênio Leal \\ Head of the Science and Engineering Center - PUC-Rio
}


All Rights Reserved.

\section{Manoel Feliciano da Silva Junior}

Graduated in Electrical Engineering from Universidade Federal de Pernambuco in 1988, Master in Mechanical Engineering from Pontifícia Universidade Católica do Rio de Janeiro in 2004 and Equipment Engineer at Petrobras.

Bibliographic Data

Silva Junior, Manoel Feliciano da

Intelligent well transient temperature signal reconstruction / Manoel Feliciano da Silva Junior ; advisor: Marcio da Silveira Carvalho ; co-advisor: Arthur Martins Barbosa Braga. - 2012.

162 f. : il. (color.) ; $30 \mathrm{~cm}$

Tese (doutorado) - Pontifícia Universidade Católica do Rio de Janeiro, Departamento de Engenharia Mecânica, 2012.

Inclui bibliografia

1. Engenharia Mecânica - Teses. 2. Intelligent well. 3. Numeric flow simulation. 4. Finite elements. 5. Data analysis. 6. Robust principal component analysis. 7. Diffusion maps. I. Carvalho, Marcio da Silveira. II. Braga, Arthur Martins Barbosa. III. Pontifícia Universidade Católica do Rio de Janeiro. Departamento de Informática. III. Título. 


\section{Acknowledgements}

It has been a very long journey since the work on this thesis started. Finally, after all the efforts it became true.

First of all, I would like to thank all my family for backing me up along the way. My wife Luciane and my two sons Manoel and Gustavo were of big importance and created a great environment for me along these years. Without them I would not have the strength to peacefully follow my path in order to make this achievement.

To my advisors Marcio Carvalho and Arthur Braga from PUC-Rio and David Davies from Heriot-Watt I would like to express all my gratitude for the everyday consideration and encouragement for the realization of this work. Their contribution and patience were immense.

I also want to give special thanks to Dr. Khafiz M Muradov who has also provided an important support and contribution for this thesis.

Further, I would like to send thanks out to all my colleagues from PUC-RIO, in particular to André T. Machado, for providing a great environment and productive discussions.

Last but certainly not least, I am very thankful for all the support given from Petrobras which made everything possible. 


\section{Abstract}

Da Silva Junior, Manoel Feliciano; advisor: Carvalho, Marcio da Silveira; co-advisor: Braga, Arthur Martins Barbosa. Intelligent Well Transient Temperature Signal Reconstruction. Rio de Janeiro, 2012. 162p. D.Sc. Thesis - Departamento de Engenharia Mecânica, Pontifícia Universidade Católica do Rio de Janeiro.

Intelligent Well (IW) technology has built-up several years' production experience. Numerous publications have described how remote flow control and monitoring capabilities can lead to fewer interventions, a reduced well count and improved reservoir management. Despite the maturity of IW equipment, the concept of the integrated IW as a key element in the "Digital Oil Field" still not fully developed. Permanent monitoring systems in this framework play an important role as source of the necessary information about actual production system aiming model calibration and uncertainty minimization. However, each extra permanently installed sensor increases the well's installation complexity and operational risk. A well-founded understanding of what data is actually needed and what analysis techniques are available to extract the required information are key factors for the success of the IW project. This work proposes a new framework to real-time data analysis through centralizing pre-processing. A numeric IW transient temperature model is developed, tested and validated to generate synthetic data. It was chosen without loss off generality as a representative application to test and validate the "cleansing" and feature extraction algorithms developed. The results achieved are compared with the state of the art ones showing advantages regarding efficiency and potential to capture mutual influence among processes.

\section{Keywords}

Intelligent Well; Numeric Flow Simulation; Finite Elements; Data Analysis; Robust Principal Component Analysis; Diffusion Maps. 


\section{Resumo}

Da Silva Junior, Manoel Feliciano; orientador: Carvalho, Marcio da Silveira; co-orientador: Braga, Arthur Martins Barbosa. Reconstrução de sinais transientes de temperatura em poços inteligentes. Rio de Janeiro, 2012. 162p. Tese de Doutorado - Departamento de Engenharia Mecânica, Pontifícia Universidade Católica do Rio de Janeiro.

A tecnologia de poços inteligentes já possui muitos anos de experiência de campo. Inúmeras publicações tem descrito como o controle de fluxo remoto e os sistemas de monitoração podem diminuir o número de intervenções, o número de poços e aumentar a eficiência do gerenciamento de reservatórios. Apesar da maturidade dos equipamentos de completação o conceito de poço inteligente integrado como um elemento chave do "Digital Oil Field" ainda não está completmente desenvolvido. Sistemas permanentes de monitoração nesse contexto tem um papel fundamental como fonte da informação a respeito do sistema de produção real visando calibração de modelos e minimização de incerteza. Entretanto, cada sensor adicional representa aumento de complexidade e de risco operacional. Um entendimento fundamentado do que realmente é necessário, dos tipos de sensores aplicáveis e quais técnicas de análises estão disponíveis para extrair as informações necessárias são pontos chave para o sucesso do projeto de um poço inteligente. Este trabalho propõe uma nova forma de tratar os dados em tempo real de poços inteligentes através da centralização do pré-processamento dos dados. Um modelo poço inteligente numérico para temperatura em regime transiente foi desenvolvido, testado e validado com a intenção de gerar dados sintéticos. A aplicação foi escolhida sem perda de generalidade como um exemplo representativo para validação dos algorítmos de "limpeza" e extração de características desenvolvidos. Os resultados mostraram aumento da eficiência quando comparados com o estado da arte e um potencial para capturar a influência mútua entre os processos de produção.

\section{Palavras Chave}

Poço Inteligente; Simulação de escoamento; Elementos Finitos; Análise de Dados; Análise de Componentes Principais Robusta; Mapas de Difusão. 


\section{Contents}

1. Introduction 20

1.1. Scope 21

1.2. Contributions 22

1.3. Outline 22

2. Intelligent Wells 23

2.1. Permanent Monitoring Systems 25

2.2. Packers 25

2.3. Inflow Control Valve 26

2.4. Lines, connectors, feedthrough connectors and clamps 27

2.5. Wet Disconnection Tool 29

2.6. Multi-point Chemical Injection $\quad 29$

2.7. Intelligent Well Control System 30

2.8. Intelligent Well Surface Control System 31

3. IW Monitoring Systems 33

3.1. Electronic Sensors $\quad 34$

3.1.1. Pressure and Temperature $\quad 34$

3.1.2. Quasi-Distributed Temperature 34

3.1.3. Single Phase and Two-Phase Flowmeter 35

3.1.4. Streaming Potential 35

3.1.5. Permanent 3D Resistivity 36

3.1.6. Permanent Downhole Seismic 36

3.2. Fiber Optic Sensors $\quad 37$

3.2.1. Pressure and Temperature, Quasi-Distributed Temperature, and Quasi-Distributed Strain Sensors 38

3.2.2. Singles Phase and Two-Phase Flowmeter 39

3.2.3. Permanent Downhole Seismic Sensors 42 
3.2.4. Distributed Temperature and Distributed Temperature and Strain Sensors

3.2.5. Distributed Acoustic/Vibration Sensors 44

3.3. Metrology and Realiability 46

3.3.1. Necessary Measurement Uncertainty and Placement Optimization

3.3.2. Reliability 50

3.4. Basic Integrated IW Monitoring System Design Framework 52

3.5. Application Areas 54

3.6. IW Monitoring System Design Example 55

4. Numeric Non-Isothermal Dynamic Well-Reservoir Model 63

4.1. Well-Reservoir Model 68

4.1.1. Reservoir Layer Model (derived in appendix D) 69

4.1.2. Well Segment Model 71

4.1.2.1. Mass Balance 71

4.1.2.2. Momentum balance 72

4.1.2.3. Temperature model 73

4.1.3. Initial and Boundary Conditions $\quad 74$

4.2. Heat transfer coefficients 75

4.2.1. Heat transfer coefficient between annulus and tubing 76

4.2.2. Heat transfer coefficient between annulus to formation 76

4.3. Inflow control valve 76

4.4. Numerical Solution 79

4.4.1. Variational Formulation 80

4.4.2. Space and time discretization 82

5. Validation and Case Studies 87

5.1. Segment Validation 87

5.1.1. Vertical Well 88

5.1.2. Cylindrical shaped reservoir 89

5.1.3. Case Studies 91

5.1.3.1. Base Case 92

5.1.3.2. Two-Phase at Middle Zone 94 
5.1.3.3. Different Permeability at Middle Zone 96

5.1.3.4. Zonal Transient 98

6. Data Analysis 102

7. Pre-processing Theory Background 108

7.1. Corrupted Measurement Data 108

7.2. Denoising and Outlier Removal 112

7.2.1. Conventional Filters 112

7.2.2. Robust PCA via Principal Component Pursuit 117

7.3. Feature Extraction 123

7.3.1. Diffusion Maps 124

7.3.1.1. Diffusion Maps Algorithm 124

7.3.1.2. Toy Example $\quad 125$

7.3.2. Feature Extraction Framework $\quad 127$

7.4. Pre-processing Framework 128

8. Pre-processing Framework Validation and Case Studies $\quad 131$

8.1. Noise, Outlier, and Signals 131

8.2. Denoising/Outlier Removal and Transient Identification 134

8.2.1. Denoising/Outlier Removal 134

8.2.2. Transient Detection 138

8.3. Validation Using Only Pressure Signal 138

8.4. Case Studies 140

8.4.1. Denoising and Outlier Removal of Transient Temperature Signals

8.4.2. Transient Detection through Pressure and Temperature signals

9. Conclusions and Future Works 143

9.1. IW Monitoring Systems Design 143

9.2. Fully Coupled Non-Isothermal Dynamic IW-Reservoir Model 143

9.3. Data Analysis Pre-Processing 144 
Bibliography

Appendix A

151

Appendix B

153

Appendix C

155

Appendix D

159

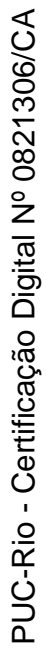




\section{Symbols}

\begin{tabular}{|c|c|c|c|}
\hline$S$ & Saturation & $R_{s}$ & Solubility ratio of phase $\mathrm{j}$ ' in phase $\mathrm{j}$ \\
\hline$T$ & Temperature & $K_{J T}$ & Joule-Thomson coefficient \\
\hline $\boldsymbol{u}$ & Velocity vector & $C_{p}$ & Mass heat capacity at constant pressure \\
\hline $\boldsymbol{u}_{g m}$ & Gas drift velocity & $A$ & Area \\
\hline $\boldsymbol{k}$ & Permeability tensor & $P$ & Potential \\
\hline$k_{r}$ & Relative permeability & $\phi$ & Porosity \\
\hline$p$ & Pressure & $c$ & Compressibility \\
\hline$q$ & Volumetric flow & $U$ & Internal energy \\
\hline$q_{T}$ & Thermal flow & $H$ & Enthalpy \\
\hline$\rho$ & Density & $K_{T}$ & Thermal conductivity \\
\hline$\mu$ & Viscosity (dynamic) & $\tau$ & Stress tensor \\
\hline$\lambda$ & Relative mobility & $\beta$ & Volumetric thermal expansivity \\
\hline$g$ & Standard gravity & $U_{w b}$ & Wellbore heat transfer coefficient \\
\hline$f_{F}$ & Fanning friction factor & $R$ & Radius \\
\hline$\theta$ & Angle with the vertical & $y_{p}$ & Volumetric fraction of the liquid phase $p$ \\
\hline$T_{e}$ & Reservoir initial temperature & $x_{p}$ & Volumetric fraction of the gas phase $p$ \\
\hline$g_{T}$ & Geothermal gradient & $\gamma$ & $\begin{array}{l}\text { Opened surface area and total surface } \\
\text { area ratio }\end{array}$ \\
\hline$\tau_{\text {wall }}$ & Shear stress at the pipe wall & $C_{o}$ & Distribution coefficient \\
\hline$D$ & Diameter & $\sigma$ & Surface tension \\
\hline$D_{h}$ & Hydraulic diameter & $F_{p}$ & Flow pattern factor \\
\hline$\varepsilon$ & Pipe roughness & $I^{*}$ & Inclination factor \\
\hline$R_{m}$ & Reynolds number of the mixture & $\operatorname{Pr}$ & Prandt number \\
\hline$R e$ & $\begin{array}{l}\text { Reynolds number per unit length of radial } \\
\text { reservoir flow }\end{array}$ & $G r$ & Grashof number \\
\hline$U_{t a}$ & $\begin{array}{l}\text { Heat transfer coefficient between tubing to } \\
\text { annulus }\end{array}$ & $R a$ & Raleigh number \\
\hline$U_{a f}$ & $\begin{array}{l}\text { Heat transfer coefficient between annulus to } \\
\text { formation }\end{array}$ & $C_{d}$ & Discharge coefficient \\
\hline $\mathrm{h}_{\text {lat,jj }}$ & Latent heat of phase j' in phase $\mathrm{j}$ & $h$ & Heat transfer coefficient \\
\hline
\end{tabular}

\section{Subscript}

\begin{tabular}{|ll|ll|}
\hline$w$ & Water component/phase & $m$ & Mixture \\
$o$ & Oil phase component/phase & $I$ & Inflow \\
$s$ & Rock & $f$ & Fluid \\
$z$ & z direction & $t$ & Tubing \\
$r$ & r direction & $a$ & Annulus \\
$p$ & Phase & & \\
\hline
\end{tabular}




\section{Acronyms and Abbreviations}

CT Christmas Tree

DAS Distributed Acoustic Sensing

DM Diffusion Maps

DTS Distributed Temperature Sensing System

DTSS Distributed Temperature and Strain Sensing System

DVS Distributed Vibration Sensing

EM Electromagnetic Method

ESP Electric Submersible Pump

FBG Fiber Bragg Grating

GLV Gas Lift Valve

GOR Gas-Oil Ratio

GVF Gas Void Fraction

ICV Inflow Control Valve

IPR Inflow Performance Relationship

IW Intelligent Well

IWC Intelligent Well Completion

IWCS Intelligent Well Control System

IWIS Intelligent Well Interface Standardization

IWSCS Intelligent Well Surface Control System

JIP Joint Industry Project

MAD Median Absolute Deviation

MD Measured Depth

MEMS Micro Electro-Mechanical Systems

MIMOSA Non-profit trade association dedicated to operations and maintenance standards

MPFM Multi-Phase Flowmeter

MTTF Mean Time To Failure

NIST National Institute of Standards and Technology

NP Non-Polynomial Time

NTP Network Time Protocol

OBC Ocean Bottom Cable

OLEDB Object Linking and Embedding Database 
OPC OLE for Process Control Connectivity

PCP Principal Component Pursuit

PDG Permanent Downhole Gauge

PES Petroleum Engineering Services

PRODML Production Markup Language

PVT Pressure-Volume-Temperature Analysis

RED Regression Estimated Decline

RESQML Reservoir Characterization Markup Language

RMAD Robust Median Absolute Deviation

RMSE Root Mean Squared Error

ROV Remote Operated Vehicle

RPCA Robust Principal Component Analysis

RTD Resistance Temperature Detectors

SCAD Smoothly Clipped Absolute Deviation

SCADA Supervisory Control and Data Acquisition System

SCM Subsea Control Module

SCRAMS Surface-Controlled Reservoir Analysis \& Management System

SHD Symmetric Hausdorff Distance

SOI Silicon on Insulator

SONAR Sound Navigation and Ranging

SoS Speed of Sound

SVD Singular Value Decomposition

TCP/IP Transmission Control Protocol/Internet Protocol

TDM Time Division Multiplex

TH Tubing Hanger

TSR Tubing Separation Tool

TTA Temperature Transient Analysis

TVD True Vertical Depth

VSP Vertical Seismic Profile

WC Water Cut

WCT Wet Christmas Tree

WDM Wavelength Division Multiplex

WH Wellhead

WITSML Wellsite information Transfer Standard Markup Language 


\section{Figures}

Figure 2.1 - IW completion example. 24

$\begin{array}{ll}\text { Figure 2.2 - ICV choke profile } & 27\end{array}$

Figure 2.3 - Control line and flatpack. 27

Figure 2.4 - Control line and flatpack. 28

Figure 2.5 - TH/WCT wet-mate feedthrough connector system for a vertical and horizontal WCT. 28

Figure 2.6 - PRODML framework. 32

Figure 3.1 - FBG sensor basic principles. 38

Figure 3.2 - Convection velocity and SoS (Speed of Sound) in k- $\omega$ plane $\quad 40$

Figure 3.3 - Backscattering based sensors. 43

Figure 3.4 - Performance chart 44

Figure 3.5 - Packer setting confirmation. 46

Figure 3.6 - Flow allocation analysis. 46

Figure 3.7 - Hydraulic fracturing performance monitoring. 46

Figure 3.8 - Metrological parameters definition and relationship. $\quad 48$

Figure 3.9 - Quantifiable source of model uncertainties and their impact. 49

Figure 3.10 - IW monitoring system design basis 53

Figure 3.11 - Structure (left), well locations (middle) and permeability distribution (right) for the PUNQS3 model. 56

Figure 3.12 - Horizontal IW completion for PUNQ S-3 production well. 56

Figure 3.13 - WC simulation results.

Figure 3.14 - Temperature in lower completion showing two and all valves open.

Figure 4.1 - Well/Reservoir mutual (Alberts, Belfroid et al. 2007)

Figure 4.2 - Sensors positions and their response in a producing horizontal well (Valiullin, Ramazanov et al. 2009) 
Figure 4.3 - Zonal intelligent well transient pressure response (Muradov and Davies 2012).

Figure 4.4 - Zonal intelligent well transient temperature response (Muradov and Davies 2012).

Figure 4.5 - Pressure and temperature transient sandface pure response in a horizontal IW.

Figure 4.6 - Well-reservoir model geometry. 68

Figure 4.7 - Cylindrical-shaped reservoir layer. 69

Figure 4.8 - Annulus and tubing in a well segment. 71

Figure 4.9 - Well-reservoir segment and its radial layers 75

Figure 4.10 - ICV 77

Figure 4.11 - Geometry used in the valve model $\left(A_{2}=A_{3}\right)$. 77

Figure 4.12 - Nonuniform quadrangular mesh. 83

Figure 5.1 - One well-reservoir layer/segment with an ICV. 87

Figure 5.2 - Temperature and pressure in a vertical well with $500 \mathrm{bpd}$ of oil production in the tubing and in the annulus. 88

Figure 5.3 - Reservoir pressure and temperature (oil production) $\quad 90$

Figure 5.4 - Reservoir pressure and temperature (oil/water production) 90

Figure 5.5 - Pressure and temperature transients for 2000 [bpd] and 1000 [bpd]. $\quad 91$

Figure 5.6 - Well-reservoir schematic for case study. 92

Figure 5.7 - Well temperature: $\mathrm{T}(\mathrm{z}, \mathrm{t}=0)$. 93

Figure 5.8 - Well temperature: $\mathrm{T}(\mathrm{z}, \mathrm{t}=3 \mathrm{~h})$. 93

Figure 5.9 - Well temperature: $\mathrm{T}(\mathrm{z}, \mathrm{t}=24 \mathrm{~h})$. 94

Figure 5.10 - Well temperature: $T(z=$ half segment, $t)$. 94

Figure 5.11 - Well temperature: $\mathrm{T}(\mathrm{z}, \mathrm{t}=0)$. 95

Figure 5.12 - Well temperature: $T(z, t=3 h)$. 95

Figure 5.13 - Well temperature: $\mathrm{T}(\mathrm{z}, \mathrm{t}=24 \mathrm{~h})$. 96

Figure 5.14 - Well temperature: $T(z=$ half segment, $t)$.

Figure 5.15 - Well temperature: $T(z, t=0)$. 97

Figure- 5.16 - Well temperature:T $(z, t=3 h) . \quad 97$

Figure 5.17 - Well temperature:T(z, $\mathrm{t}=24 \mathrm{~h})$. 98

Figure 5.18 - Well temperature:T( $\mathrm{z}=$ half segment, $\mathrm{t})$. 
Figure 5.19 - Well temperature: $\mathrm{T}(\mathrm{z}, \mathrm{t}=0 \mathrm{~h})$.

Figure 5.20 - Well temperature: $T(z, t=3 h)$.

Figure 6.1 - Integrated modeling and optimization framework. 102

Figure 6.2 - Value chain pyramid chart.

103

Figure 6.3 - Data analysis framework with centralized pre-processing. 104

Figure 6.4 - Integrated IW architecture. 105

Figure 6.5 - Integrated IW application with centralized pre-processing. 107

Figure 7.1 - Random noise.

108

Figure 7.2 - Systematic errors.

Figure 7.3 - Noise and drift of a real PDG (the green signal is the "clean" one).

Figure 7.4 - Temperature gradient profile error due to reading unit noise model in a real temperature measurement (blue line without correction).

Figure 7.5 - Mean filter performance (red is the clean signal).

Figure 7.6 - Median filter performance (red is the clean signal).

Figure 7.7 - Maximum-minimum filter performance (red is the clean signal).

Figure 7.8 - Moving-average filter performance (red is the clean signal).

Figure 7.9 - Hard and soft shrinkage operator.

Figure 7.10 - Wavelet filter performance for the hard threshold (red is the clean signal).

Figure 7.11 - Wavelet filter performance for the soft threshold (red is the clean signal).

Figure 7.12 - Outliers problem.

Figure 7.13 - PCP as a denoising technique.

Figure 7.14 - Synthetic example of the inexact augmented Lagrange multiplier RPCA algorithm.

Figure 7.15 - $\operatorname{Rank}(\mathrm{L})$ and $\operatorname{Card}(\mathrm{O})$ as function of the iteration. 
Figure 7.17 - Denoising procedure in the pre-processing framework 123

Figure 7.18 - Trefoil knot with Gaussian noise. 126

Figure 7.19 - Trefoil knot with Gaussian noise. 127

Figure 7.20 - Trefoil knot with Gaussian noise. 128

Figure 7.21 - Centralized pre-processing framework. 129

Figure 8.1 - Injector-producer pair pressure signal (dark blue and light blue are lower and upper zones respectively; black producer well).

Figure 8.2 - Injector-producer pair temperature signal (red and green are lower and upper zones respectively; pink producer well). 132 Figure 8.3 - Pressure and temperature signals 1,2 and 3. Figure 8.4 - Outlier removal and denoising in Olsen's Work. 134 Figure 8.5 - Bi-orthogonal Spline39 wavelet functions. 136 Figure 8.6 - Transient detection approach based on mask-matching pattern recognition.

Figure A.1 - Shear stress at the pipe wall.

Figure A.2 - Chen (1979) - Fanning friction factor.

Figure C.1 - Heat transfer within wellbore.

Figure D.1 - Cylindrical-shaped reservoir.

Figure D.2 - Control volume of the reservoir mass balance and flow equations.

Figure D.3 - Volume control of the reservoir energy balance. 


\section{Tables}

Table 3.1 - Seismic sensors comparison by technology. 42

Table 3.2 - Typical metrological data for downhole sensors 61

Table 3.3 - Measurement types and application areas 62

Table 4.1 - IC and BC for layer 1

Table 4.2 - IC and BC for layer j 75

Table 8.1 - Bi-orthogonal Spline39 scaling filters. 136

Table 8.2 - Non-orthogonal Spline4246 scaling and wavelet filters. 137

Table 8.3 - Denoising/outlier removal and transient identification performance results.

Table 8.4 - Denoising/outlier removal and transient identification performance results using transient temperature signal.

Table 8.5 - Denoising/outlier removal and transient identification performance results using pressure and temperature signals. 
"The dictionary is the only place where success comes before work." Vince Lombardi 


\section{1}

\section{Introduction}

IW (Intelligent Well) technology has been built-up several years of production experience. Numerous publications have described how remote flow control and monitoring capabilities can lead to fewer interventions, a reduced well count and improved reservoir management. Despite the maturity of IW equipment, the concept of the integrated IW as a key element in the "Digital Oil Field" is still not fully developed. Today's practice consists of evaluating the IW value chain in a "fit-for-purpose" manner rather than using an integrated modeling and optimization framework.

Design considerations of an IW project requires a standard framework to evaluate, select, define, execute and operate the project and systematically integrate the contributions from all disciplines involved (Lau 2008). This means that all connected physical components such as reservoir, wells and production facilities have to be taken into account. Additionally, over simplification in the integrated modeling assessments should be avoided and consistent treatment of all important parameters of the production system should be provided (Hudson, Alves et al. 2011).

Permanent monitoring systems in this framework play an important role as source of the necessary information about actual production system aiming at model calibration and uncertainty minimization. However, each extra permanently installed sensor increases the well's installation complexity and operational risk. A well-founded understanding of what data is actually needed and what data analysis techniques should be available to extract the required information are key factors for the success of the IW project.

There are a variety of robust, commercial and in-house data analysis tools available to the operator (IW applications included). The challenge is to compare and integrate the results from these tools in a way that a reliable decision can be made. The integrated approach calls for the operator's chosen models to be fully integrated (from reservoir, wells/flowlines/risers to topside facilities). There is no off-the-shelf solution which efficiently mimics all the physical flow systems described above. Neither there is a set of applications which honors the needs of 
the different disciplines without important simplifications that result in losing the incremental value of the integrated modeling and optimization framework (Hudson, Alves et al. 2011). A new data analysis approach with a centralized preprocessing is proposed aiming to design a robust permanent monitoring architecture to deliver the full "Added Value" of the IW technology. It also allows feature extraction that can potentially improve the quality of the post-processing and interpretation predictions. This new approach has not yet been fully explored, even though a successful application can be found in offshore production and maintenance operations (Friedemann, Varma et al. 2008).

A numeric non-isothermal dynamic well-reservoir model is developed and validated as an IW representative application to generate synthetic data and illustrate the applicability of this new data analysis approach. It combines the reservoir temperature model from (Sui, Zhu et al. 2008) and the well temperature model from (Muradov and Davies 2008) including the necessary modifications for the two phase flow formulation and multilayer reservoir.

The signal reconstruction algorithms can be used with single point, multipoint, and distributed/quasi-distributed temperature monitoring systems. The idea is identifying the impact of the temporal and spatial resolution as well as noise and outlier in the new data analysis framework proposed. Two algorithms are used: robust principal component analysis (Candès, Li et al. 2011) for denoising and outlier removal and DM (Diffusion Maps) (Coifman and Lafon 2006) for feature extraction. The results are compared with the ones from (Olsen 2011) showing in which cases there were improvements. Compression and time synchronization are discussed but they are suggested as future work.

\section{1}

Scope

The goal of this thesis is to develop and study a new data analysis framework with centralized pre-processing. The study was limited to a temperature transient application as a representative example without loss of generality. 


\section{2}

\section{Contributions}

The contributions of this thesis can be summarized as:

- In-well monitoring system design framework with integrated modeling and optimization in mind;

- Numerical non-isothermal dynamic well-reservoir model for multi-layer reservoir and vertical well equipped with inflow control valves;

- Data analysis pre-processing techniques for denoising, outlier removal and feature extraction for in-well measurements based on robust principal component analysis and DM respectively.

\section{3 \\ Outline}

The organization of this thesis can be summarized as follows:

Chapter 2 reviews intelligent completion technology and introduces the concept of integrated intelligent completion.

Chapter 3 reviews permanent monitoring systems and introduces a new IW monitoring system design framework. The benefit of dealing with data quality control is presented.

Chapter 4 describes the numerical non-isothermal dynamic well-reservoir model for multi-layer reservoir and vertical wells equipped with inflow control valves and its practical implementation.

Chapter 5 validates the model described in chapter 4 and analyses temperature transients caused by step-like change of downhole flow control devices.

Chapter 6 reviews data analysis and interpretation concepts and proposes a new data analysis framework with centralized pre-processing.

Chapter 7 reviews theoretical background for denoising, outlier removal and feature extraction based on robust principal component analysis and DM.

Chapter 8 validates and compares the denoising and feature extraction algorithms with the state of the art ones.

We conclude our research with a summary of our findings and a discussion for future work in Chapter 9. 


\section{2 \\ Intelligent Wells}

A new completion technology named SCRAMS (Surface-Controlled Reservoir Analysis \& Management System) was developed during a JIP (Joint Industry Project) in the mid-nineties by Shell and PES (Petroleum Engineering Services)/Halliburton for saving cost associated with routine production logging and planned operations to manage commingle reservoirs production. In 1997 SCRAMS had its first installation in North Sea and it was claimed to be the world's first "smart" well completion system. Right afterwards Halliburton replaced the technology name from SCRAMS to SmartWell ${ }^{\circledR}$. IW or IWC (Intelligent Well Completion) has been adopted by the market since then to avoid trademark issues. Despite the name used a well equipped with intelligent elements which adds value to the well project during its lifecycle is the most accepted definition of IW today.

The main objective of production optimization at field scale using "Digital Oil Field" concepts is to maximize production and recovery focusing on safety and environment. In this scenario, reservoir management becomes a multidisciplinary effort where IW as a key element of the "Digital Oil Field" is still under development. Today's practice consists of evaluating IW value chain in a "fit-for-purpose" manner rather than using an integrated modeling and optimization framework to select, define, execute and operate the IW project and systematically integrate the contributions from all disciplines involved (Lau 2008). Additionally, an integrated production modeling which takes into account all connected physical components such as reservoir, wells and production facilities have to be used. The workflow should avoid over simplification in the integrated modeling assessments and provide a consistent treatment of all important parameters of the production system (Hudson, Alves et al. 2011).

Well completion design as consequence of this new framework is a challenging task since today's technology has resulted in a wide variety of hardware and software being employed to manage the current well stock. Relatively cheaper new hybrid (electric/hydraulic) IW systems are becoming available, enabling the flow control system and the sensor to share the same 
system architecture. This represents a reduced number of control lines and allows interchangeable modules for actuators and sensors, increasing the installation flexibility while simplifying the installation procedure. The end result is less risky operations with equipment suitable for a broader range of applications.

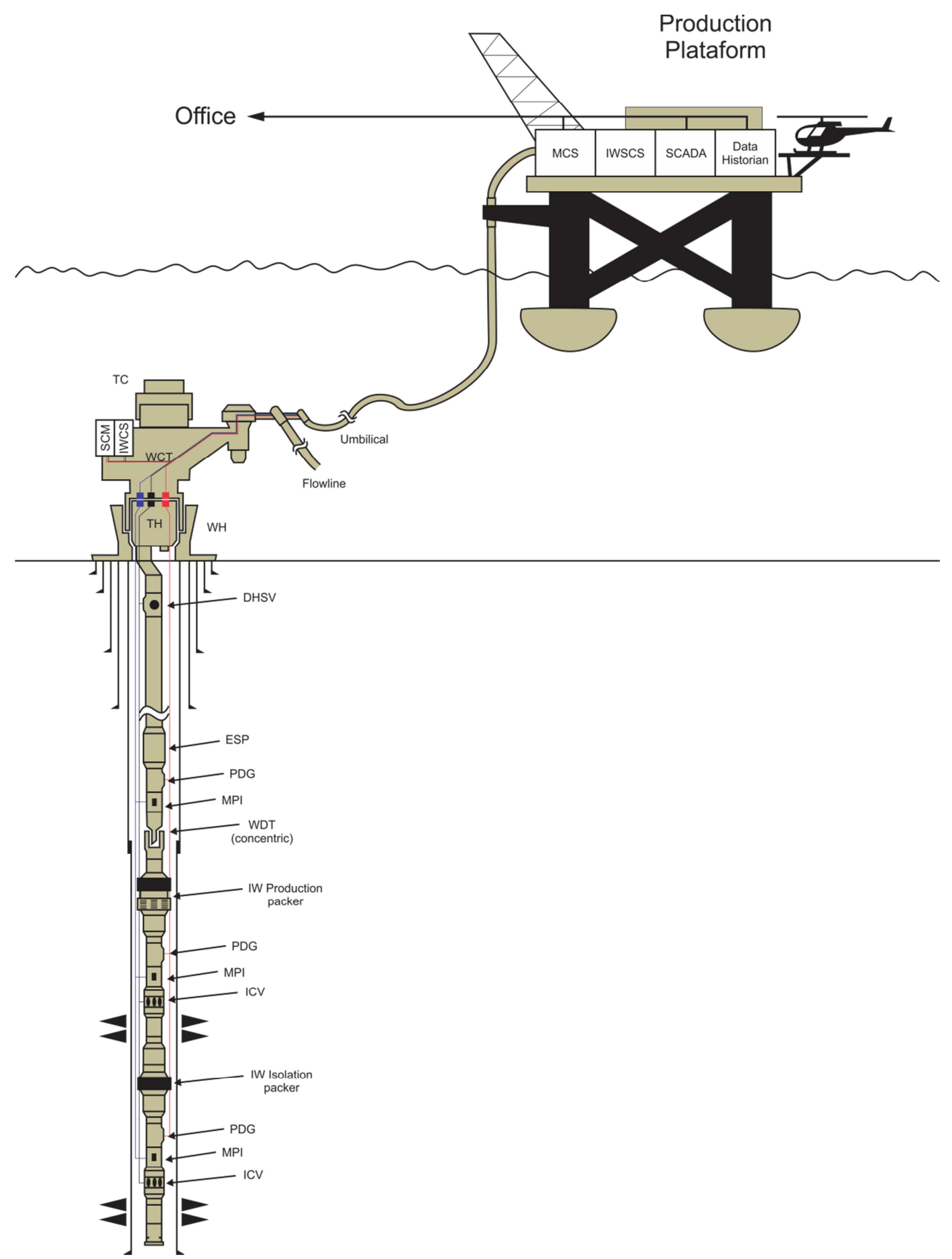

Figure 2.1 - System view of the IW in a subsea environment. 
This chapter has been written as an overview of the IW completion elements, design considerations and installation requirements according to an integrated modeling and optimization framework in mind. An IW completion in this context can be seen as actuators and sensors of a control system where the reservoir is the process to be controlled. Figure 2.1 illustrates this idea in subsea environment.

\section{1}

\section{Permanent Monitoring Systems}

IW monitoring systems continuously evolve. They started with the basic measurements of the conventional PDG (Permanent Downhole Gauge) that was only able to react to changes within the wellbore. Nowadays they can be equipped with a wide range of sensors which enable them to look deep into the reservoir and/or identify more subtle effects. This brings additional value to the IWs, being able to control zonal production/injection proactively, reduce the production uncertainty and provide data for improving the reservoir models.

Next chapter will review IW monitoring systems, their availability, applicability and limitations. It will discuss data acquisition issues in-depth; e.g. resolution, data processing and reliability. In addition, an example of an integrated IW application is given.

\section{2 \\ Packers}

IW packers are used to provide hydraulic isolation of zones which can be different reservoirs or different layers of the same reservoir allowing selective control; the uppermost completion packer is also responsible for anchoring the tubing and providing the first safety barrier for the annulus, the same basic functions of the conventional production packer. There are also packers for isolation purposes that have only isolating material with no anchoring which reduces the force needed for unsetting them and allows a large number of isolation intervals. The IW packers also have feedthroughs to bypass control lines; typically an IW packer presents from four to nine feedthroughs. During the setting procedures, the IW packer must not have any relative movement of its components so it does not transmit any tension to the control lines. 


\section{3 \\ Inflow Control Valve}

Inflow control valves are responsible for allowing selective control of production or injection. They can be actuated hydraulically, electrically or through a combination of both (multiplexed). Even though the control of ICVs (Inflow Control Valves) has been changed towards only hydraulic systems due to its reliability and cost, nowadays there is a trend back to electrical and downhole multiplexed systems. Mainly due to reliability improvement in downhole electronics and number of feedthroughs restrictions in tubing hanger and WCT (Wet Christmas Tree) for subsea production. The most common control type is still hydraulically actuated, which uses one opening control line for each valve and a common close control line for the system to reduce the number of hydraulic control lines installed. In electrically actuated valves, an electrical motor is responsible for the sleeve movement. The motors are actuated using one single electrical control line for all motors that supplies also the addressing information, which is decoded in each valve. The electric/hydraulic systems use an electric control line for multiplexing and one or more hydraulic control lines to provide power to move the sleeve. The valves can also be classified according to the flow control they provide as on-off, multiposition and infinitely variable. The on-off valves only provide the selectivity by allowing or not the flow. The multiposition valves provide several steps of choking, and are designed accordingly to the flow rate expected on the well. They can use an index system to restrict the course and provide the choking or an external device that provides a very controlled volume of hydraulic fluid in each shifting. The infinitely variable valves are more complex and require feedback on their position to adjust the correct choking. There are also different geometries for the valve orifices, but the most common are circular and variable area slots, which are used in on-off/multiposition and infinitely variable respectively. It is worth to mention that inflow control valves not only control flow from annulus to tubing but also control flow from tubing to tubing using its shrouded version. The main parameters on specifying IW valves are: number of choking positions, choking profile, flow range, maximum pressure and maximum differential pressure. 


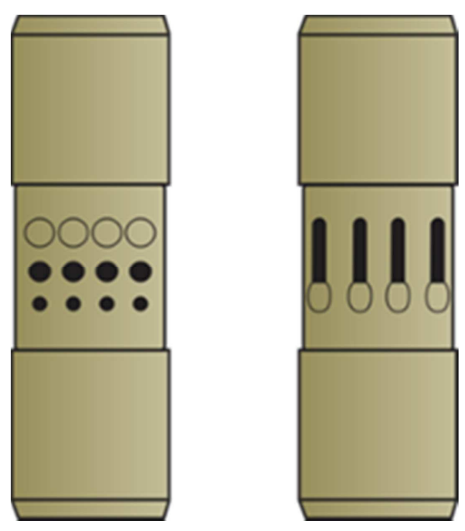

Figure 2.2 - ICV choke profile

\section{4}

\section{Lines, connectors, feedthrough connectors and clamps}

IW requires control lines to remotely control its components and receive data from the sensors. These lines have to be protected against shocks and chemical attacks during intervention procedures. Industry standard 1/4" lines are generally used to offer mechanical resistance and a polymeric material to provide chemical protection. To improve the support resistance, armor cables can also be used alongside the lines, forming a flatpack. In subsea IW applications the electrical control lines are limited to single wire or twisted pair due to tubing hanger wet-mate feedthrough connector system. It is recommended to use the twisted pair electrical control line due to its noise characteristics and improvements in telemetry to handle multiple sensors at faster data rate. Fiber optic applications have at least one fiber available (typically three) and there are mechanical restrictions in the TH/WCT connector system. Subsea fiber optic applications up to the writing of this thesis are not available due to TH/WCT wetmate connector system and downhole wet-mate connector.

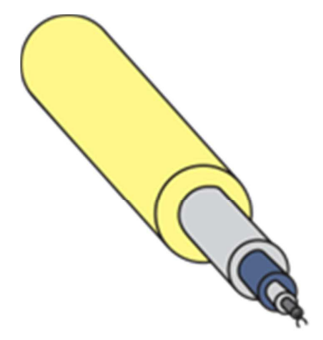

single control line

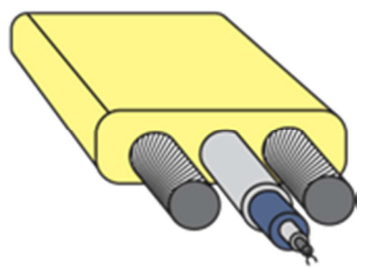

flatpack

Figure 2.3 - Control line and flatpack.

Control lines protectors - clamps - are used to hold the lines to the production tubing and to offer extra protection to it near the tubing couplings, where the diameter of the tubing is increased. 

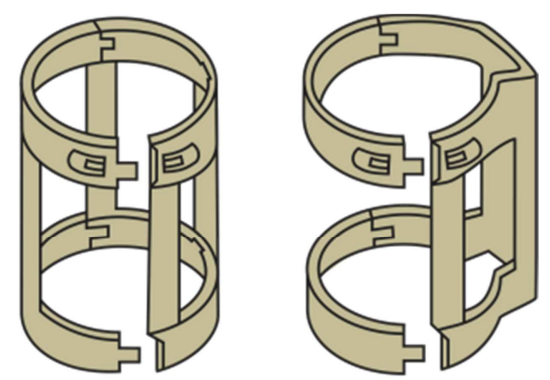

single control line flatpack

Figure 2.4 - Control line protectors (Clamps).

Dry-mate connectors are used to connect a control line to a sensor or another control line. To pass the signal through the $\mathrm{TH}$ and $\mathrm{WH}$, wet-mate feedthroughs connectors are used to ensure that there is no pressure communication. In subsea trees or downhole, wet-mate connectors are used in seawater environments or in the presence of production fluids. There is a range of electrical connectors, both dry and wet-mate, and feedthroughs qualified for vertical and horizontal WCT commercially available. It is recommended the use of single pin two contacts $\mathrm{TH}$ wet-mate feedthrough system due to the reasons explained earlier in this section. This is not the case for the fiber optic counterpart where there is only an advanced stage of development to date for the TH/WCT wet-mate feedthrough connector system and still no reliable solution for the downhole wet-mate connector. Even though, dry mate and ROV (Remote Operated Vehicle) wet-mate connectors are qualified and commercially available.
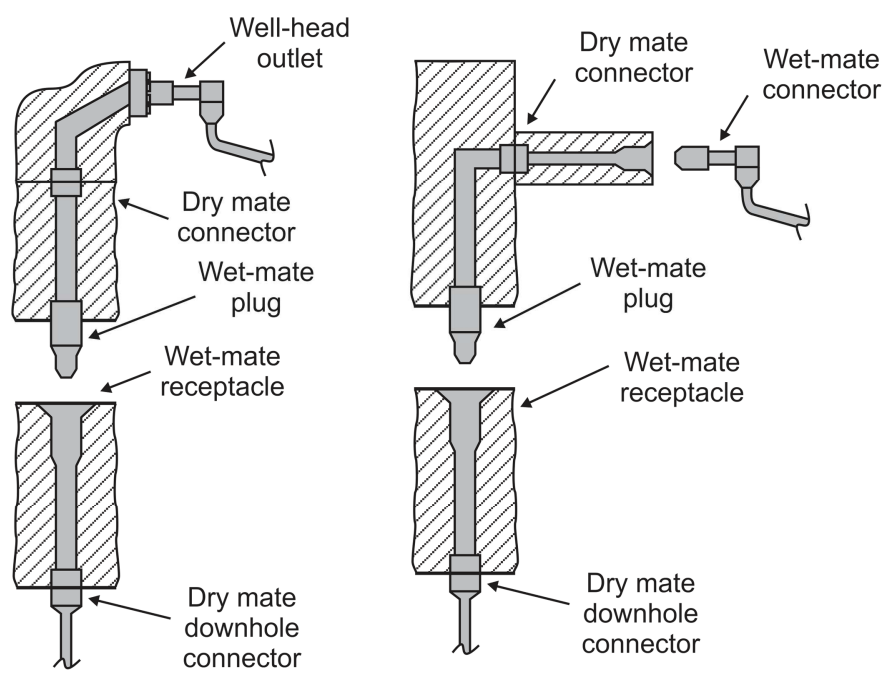

Figure 2.5 - TH/WCT wet-mate feedthrough connector system for vertical and horizontal WCTs. 


\section{5 \\ Wet Disconnection Tool}

IW is used integrated with other completion tools such as artificial lift and sand control. Typically when there are reliability issues between upper and lower completion a specific tool named WDT (Wet Disconnection Tool) is necessary. It enables upper completion to be disconnected from lower completion and reconnected afterwards for maintenance purposes. An artificial lift using a high performance ESP (Electric Submersible Pump) which has lower MTBF (Mean Time Between Failure) is a representative example of the reliability issues mentioned. Basically it is a TSR (Tubing Separation Tool) equipped with control lines wet-mate connectors available to hydraulic lines and electric lines but still in development to fiber optic lines. Advances, however, have been made do avoid orientation requirement and expand the number of channels being available. Based on the connection principle it can be classified as discrete or concentric as can be seen in Figure 2.6. The former uses conventional wet-mate connectors and the latter and a manufacturer specific design.

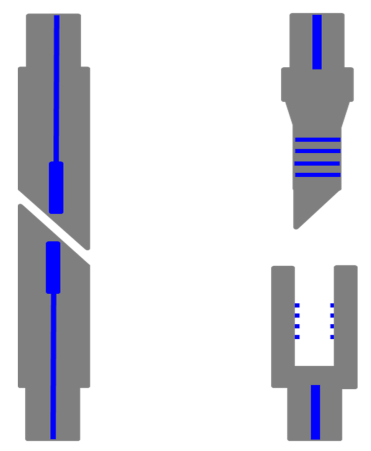

Figure 2.6 - Discrete and concentric WDTs respectively.

\section{6 \\ Multi-point Chemical Injection}

A typical downhole chemical injection system consists of a chemical control line and a chemical injection mandrel with a valve installed as part of the production tubing string. Usually a check valve is also installed at the point of injection to prevent flow from the production tubing to the injection flow path. A high-pressure pump, capable of overcoming the tubing pressure, is installed on the surface to pump fluid into the downhole point of injection. They are used for scaling control as water cut or condensate water increases in oil wells and gas 
wells respectively. Other chemicals injected downhole may be corrosion inhibitors and hydrate inhibitors for gas wells.

IW completion is sensitive to scaling for proper operation due to its moveable parts. Installing a chemical injection system in this case should be multi-point to guarantee continuous chemicals flow in the annulus of each open zone and no chemicals flow in the closed zones. In this scenario it is important the complete integration between the IW and chemical injection system reinforcing the idea of common architecture IW systems mentioned in the beginning of this chapter.

\section{7 \\ Intelligent Well Control System}

Subsea IW is controlled and monitored by a production SCM (Subsea Control Module) installed on a WCT or a manifold. The SCM provides one or more hydraulic control lines to operate the downhole safety valves and inflow control valves as well as electrical/optical control lines to the downhole sensors in the completion. Umbilical and in-field jumpers supply normally low/high hydraulic pressure, electrical power and communications to SCM which come from topside. This configuration is presented in the IWIS (Intelligent Well Interface Standardization) addendum of the ISO 132628-6 as option 1 configuration 1. The first impression of this is a fully integrated system, less complex with a reduced cost. To date this is difficult to accomplish due to the lack of a full compatible set of interfaces (mechanical, electric, protocols, hydraulic power, etc.) leading to time consuming and costly custom solutions with impacted reliability. In mature fields, upgrades to install IW in this way are even worse. In addition, IW systems are becoming more complex and adding new sensors and functions to face the integrated framework challenges, which mean continuous changes. The recommended solution for subsea IWCS (Intelligent Well Control System) to achieve reliable solutions is the use of IWIS option 3 configuration 2 as detailed in IWIS Recommended Practice A2. This kind of solution allows production control and IWCS tests to be done independently and faster introduction of new technologies with reduced risk. At the operation phase, it also makes the management of responsibilities clear. Besides IW interfaces standardization there are two more efforts in this direction (standardization) that is 
worth to mention: SIIS (Subsea Instrument Interface Standardization) and SEAFOM (Subsea Fiber Optic Monitoring). Improvements in subsea instrumentation reliability and promoting fiber optic instrumentation in subsea applications are the goals of them respectively.

\section{8 \\ Intelligent Well Surface Control System}

IW surface control system is generally integrated to the field process network through an application layer instead of a data layer. This means that a proprietary SCADA (Supervisory Control and Data Acquisition System) is being used by the IW supplier and might be a problem to integrate with the existing one in the production unit. Due to the variety of suppliers and equipment involved, the connectivity between them should be addressed at the project phase. Open protocol specification should be adopted to simplify the project by reducing the need of additional protocol translation. Among open protocols that have been used in IWSCS (Intelligent Well Surface Control System) we should highlight OPC (OLE for Process Control Connectivity) and TCP/IP(Transmission Control Protocol/Internet Protocol) running over industrial Ethernet as the most accepted and aligned with the standardization efforts mentioned earlier. Therefore, the improved interoperability can provide the same information and services even when a change of supplier is needed. In the integrated framework scenario high levels of instrumentation led to demand for improvements in the timing and format of the provided data which is important for all optimization processes. The result was an effort of several companies for creating a high level protocol called PRODML (Production Markup Language) that comes to try solving the data exchange problem through standardization. Other solutions based on OLEDB (Object Linking and Embedding Database) and historian servers are also available but PRODML framework seems to be stronger and supported by almost all major operators and service companies. In Figure 2.7, is possible to see its scope which includes: drilling, completion and interventions (WITSML - Wellsite information Transfer Standard Markup Language); maintenance (MIMOSA - Non-profit trade association dedicated to operations and maintenance standards), SCADA and reservoir characterization (RESQML - Reservoir Characterization Markup Language). 


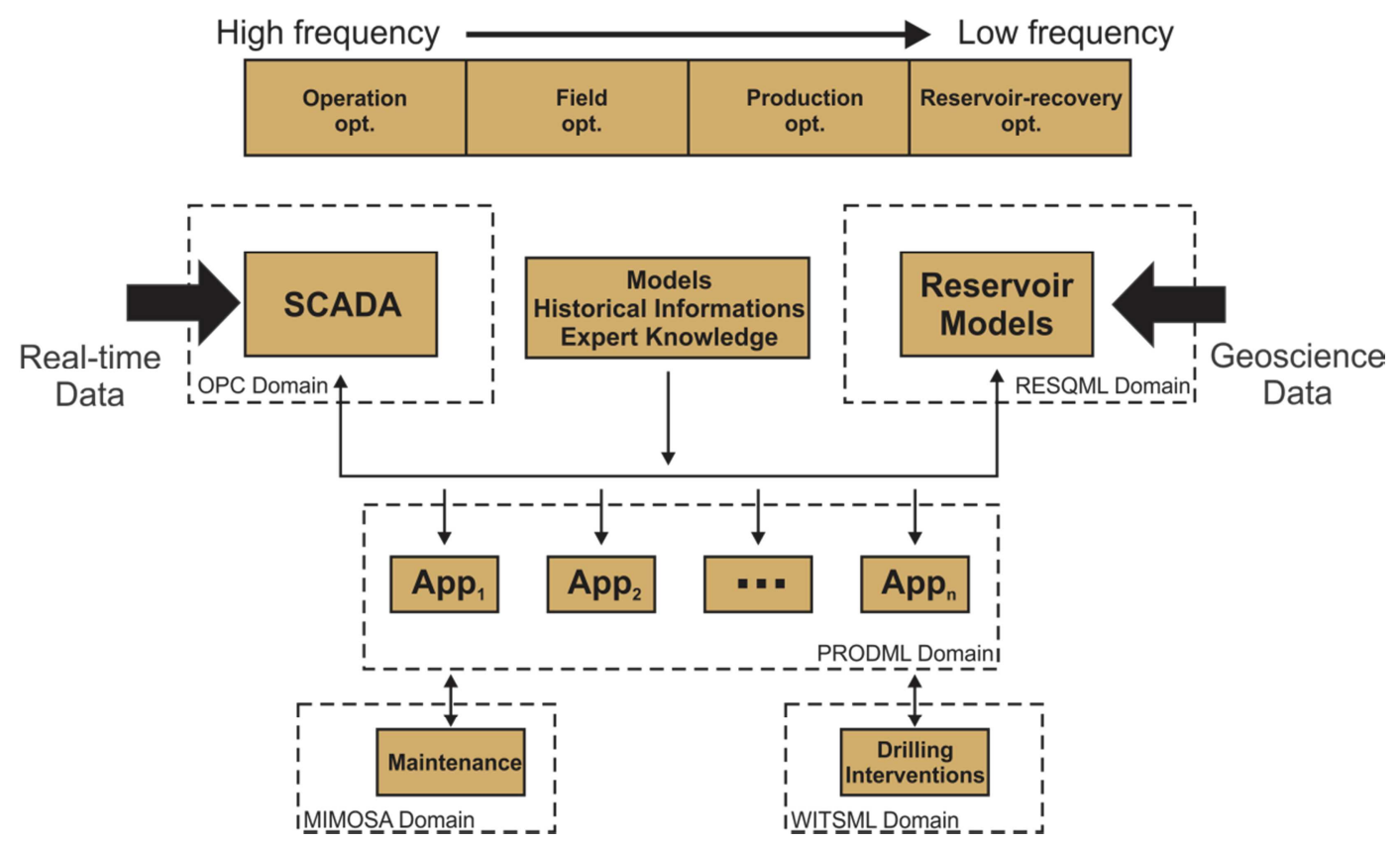

Figure 2.7 - PRODML framework.

The real-time data comes from the IW monitoring system through the IWSCS becoming available to the applications or to the centralized pre-processing proposed in this thesis and discussed in the chapter 6. The following chapter reviews the IW monitoring system introducing a new design framework. 


\section{3 \\ IW Monitoring Systems}

The physical quantities actually measured by downhole permanent monitoring systems and IW monitoring systems are almost the same. The differences and limitations between them are mostly related to the equipment and its installation requirements. They are divided in two classes: deep reservoir and near wellbore monitoring systems. Deep reservoir sensing is related to 4D seismic (time-lapse seismic), dynamic 3D resistivity and streaming potential (electrokinetic), or, in other words, monitoring techniques which capture the dynamics of the entire reservoir. Near wellbore sensing includes the classical downhole measurements such as pressure and temperature.

Permanent downhole sensors are usually classified by technology (electronic or optical) and the number of monitored points (single point, quasidistributed and distributed). Single point sensors read the physical quantity to be monitored at one point. For example, pressure is often monitored close to the reservoir depth or at the top of the interval of interest. The PDG is an example of such sensor. Quasi-distributed sensors allow the monitoring of the physical quantities at a distinct number of positions across the reservoir or interval of interest. Note that a three-zone completion with one pressure and one temperature sensor installed at each reservoir interval does not represent a quasi-distributed system; this requires at least three sensors measuring the same physical quantity to be installed at various points across each interval. Distributed sensors take this approach further by monitoring the same physical quantity at a spatial resolution as small as $0.5 \mathrm{~m}$. The DTS (Distributed Temperature Sensor) is an example of such sensor.

Permanent monitoring systems availability, applicability and limitations are reviewed in the following sections. Metrology and reliability are also addressed. An example of an application using the Integrated IW concept completes this chapter. 


\section{1}

\section{Electronic Sensors}

\subsection{1}

\section{Pressure and Temperature}

Electronic sensors have been used for more than 20 years in PDGs for measuring pressure and temperature. Quartz Resonator technology completely dominates the single point sensor market. It has been responsible for increasing pressure and temperature sensor reliability since the early nineties (Eck 1999/2000). MEMS (Micro Electro-Mechanical Systems) and the associated SOI (Silicon on Insulator) technology has been responsible for the recent advances in sensor reliability when installed in a High Pressure and High Temperature (HPHT) environment. MEMS also have shown promise in the development of sensors for measuring new physical quantities. Typical pressure and temperature sensor data is shown in Table 3.2.

The mechanical protection, the type of telemetry employed and the available number of transducers for each sensor depend on the manufacturer and the price. Commercial sensors are typically capable of having up to 4 sensors on the same electric control line (1/4 inch tubing with one twisted pair).

\subsection{2 \\ Quasi-Distributed Temperature}

Increasing the number of sensor systems makes the installation more complex and time consuming, hence less suited for downhole installation due to the increasing level of operational risk. New multiplex techniques have allowed the installation of a larger number of smaller temperature sensors on the same cable without the need for multiple connectors and mandrels. For example, up to 48 temperature measurement sensors on a single cable using high resolution, RTD (Resistance Temperature Detectors) are now commercially available (Gambhir, Shrivastav et al. 2008).

Typical sensor data is shown in Table 3.2. 


\subsection{3}

\section{Single Phase and Two-Phase Flowmeter}

Differential pressure flowmeters based on the Venturi principle are commercially available for IW applications. They depend on the differential pressure, a quantity easily measured by two pressure sensors. Flowmeters based on the Venturi principle are best suited to single-phase measurements (e.g. water injection). Their measurement uncertainty is $< \pm 1.5 \%$ (of the average) with a rangeability of 4 to 1 (ratio of the maximum and minimum flow rates that can be accurately measured). Their application to production wells is more restricted since the fluid's flow regime and the erosion velocity in the Venturi's throat can both compromise the measurement.

By contrast, an inverted Venturi is suited to high flowrates and has been commercialized as a fullbore flowmeter (Ong, Aymond et al. 2007). It provides the volumetric flow rate in the tubing together with temperature and pressure measurements in the annulus and tubing. Some manufacturers provide calculated measurements, such as WC and density, by including a densitometer or a gradiometer. They can achieve an uncertainty of $< \pm 5 \%$, depending on the PVT (Pressure-Volume-Temperature Analysis) data uncertainty (Kiryushkina, Sikandar et al. 2011). An insertable Venturi has an operational advantage if low cost interventions can be carried out by wireline, making it only a practical option for conventional wells with dry trees.

\subsection{4 Streaming Potential}

A common logging tool used to characterize reservoir properties is the spontaneous potential log. The streaming potential (one of the component of the spontaneous potential) is strongly influenced by the resistivity of the fluids and the differential pressure resulting from fluid flow through the porous media towards the well. Laboratory results and numerical experiments have shown that one can measure strong signals (up to $100 \mathrm{mV}$ ) in production wells when low salinity formation water is present (Jackson, Vinogradov et al. 2011). It should be possible to interpret those measurements to detect reservoir fluid saturation changes, e.g. due to encroaching water, at distances of tens or even hundreds of meters from the well (Jaafar, Jackson et al. 2009). Closed-loop reservoir 
management using a proactive reservoir control strategy instead of today's simulation model strategy would become practical if this promising development bears fruit.

\subsection{5}

\section{Permanent 3D Resistivity}

The EM (Electromagnetic Method) provides a signal which is proportional to the conductivity/resistivity of the formation and the surroundings. It is obtained through the induction of electrical eddy currents by an alternating magnetic field, a phenomena that responds to fluid in the formation pore space. This behavior is complementary to seismic measurements (see below) which mainly respond to impedance changes in layer boundaries. It is significantly easier to identify such changes in a time-lapse manner than the detection of the actual fluid itself. This monitoring system has a range of $0.3 \mathrm{~km}$ for fluid fronts showing a large resistivity contrast (Zhou, Julander et al. 2002). No long-term case histories have been reported only feasibility studies and field trials. These studies have trialed surface-to-borehole and borehole-to-borehole time-lapse EM sensitivity, and single well measurements. The latter case requires the ability to measure pico volts downhole which is currently a technological gap (Strack 2010).

\subsection{6 \\ Permanent Downhole Seismic}

Improvements in time-lapse 3D seismic technology have remarkably improved the quality of reservoir images. Borehole and surface seismic can define geometric boundaries with reasonable accuracy, but not the fluid content, at long distances (Strack 2010). The latter is best measured by the electromagnetic technique. The downhole seismic measurements are performed by time-lapse VSP (Vertical Seismic Profile) for borehole-to-borehole seismic imaging and by surface and OBC (Ocean Bottom Cable) seismic calibration for permanent or temporary deployment. During the production phase it can be used to passively monitor rock fracturing from both injection and production activity. A typical multilevel, downhole seismic monitoring systems has up to 30 nodes with 4 channels/node (3c geophones +1 hydrophone for multiples correction) operating 
with a sampling rate varying from 4 to $0.25 \mathrm{~ms}$. Correct coupling with the formation is essential for this type of system (Wilson, Floch et al. 2008).

MEMS accelerometers are now replacing the older electromagnetic geophones due to their higher dynamic range, broadband linear phase/amplitude response, small size and weight; though they still need improvement to minimize noise at low frequencies (Mougenot, Cherepovskiy et al. 2011). The specification of a downhole seismic accelerometer should consider the noise floor, linearity, dynamic range and (multi-component) cross-axis sensitivity. Resonant frequency should also be included since the sensor's upper useful frequency is a fraction of its resonant frequency. It thus determines the sensitivity and displacement per $g$ of acceleration.

Typical 3c geophone data and the equation required to relate the geophone's electrical and mechanical data are given in Table 3.2.

Permanent downhole seismic has not been installed in an IW completion todate. The extra cost and complexity of equipping an IW with permanent downhole seismic needs to be justified by the "Added Value" of, for example, enabling proactive control of the reservoir. Current technical developments in the combination of seismic and EM methods may make this feasible.

\section{2 \\ Fiber Optic Sensors}

Fiber optic sensors have been applied to permanent downhole monitoring systems since the mid-nineties (Zisk 2005). Initially only DTS was adopted by operators for temporary well temperature profile logging. It was only at the end of the nineties with the development of FBG (Fiber Bragg Grating) technology that fiber optic sensing started to be used in permanent downhole monitoring systems because FBG gave greater reliability and had the potential to measure a variety of physical quantities in the same cable. Single point pressure and temperature sensors, flowmeters and 3C geophones are now all commercially available at a similar level of operational complexity and cost as their electronic counterpart, but with a claimed higher level of reliability. Fiber optic's great advantage for sensing is its distributed capability, even though electronic quasi-distributed sensors can now incorporate a larger number of sensors on the same cable $(>45)$ which raise questions about comparisons of those systems. 
The technological gap for fiber optic sensing remains a reliable tubing hanger wet-mate connector for subsea deepwater applications and a downhole wet-mate connector for completions with multi trip installation or upper completion equipment that needs to be changed regularly. For example, high rate electric submersible pumps which have to be replaced at a frequency greater than the remainder of the completion.

The next sections describe the fiber optic sensors available, their metrological specifications and the basic principles of the technology that has been exploited for permanent downhole monitoring: FBG, Raman and Brillouin backscattering, coherent Rayleigh backscattering and distributed polarimetry.

\subsection{1}

Pressure and Temperature, Quasi-Distributed Temperature, and Quasi-Distributed Strain Sensors

The fiber optic alternative to electronic single point and quasi-distributed sensors is based on FBG technology. An FBG is produced by forming a periodic structure with a permanent change in the refraction index along the propagation axis of the fiber optic (Culshaw and Kersey 2008). The frequency reflected by this periodic structure depends on the optical period of the grating, which is itself a function of temperature and strain (Figure 3.1). The change in the optical path length is of the order of $1 \mathrm{ppm} /{ }^{\circ} \mathrm{C}$ (CT) and $10 \mathrm{ppm} / \mu \varepsilon$ (Cs). Downhole application of this technology faces the normal challenge of packaging and long term stability.

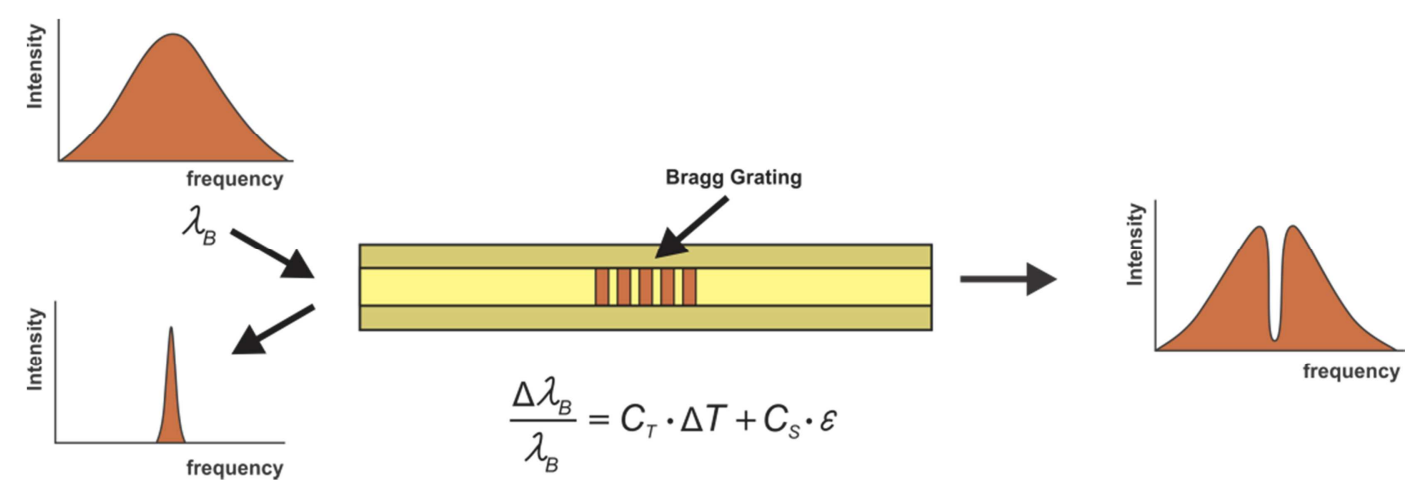

Figure 3.1 - FBG sensor basic principles.

Commercially available installations for dry trees can have up to four pressure and temperature sensors per fiber with three optical fibers per control line. Such completions have been successful in high rate gas wells due to 
reliability issues with their electronic counterpart in this harsh application with levels of vibration under producing conditions.

Typical fiber optic pressure and temperature sensor data can be seen in Table 3.2.

Quasi-distributed sensors based on FBG technology are also available for temperature and strain measurements. The FBG sensors can be easily multiplexed by WDM (Wavelength Division Multiplex). TDM (Time Division Multiplex) may be included when the number of sensors increases up to a hundred, but with a loss of accuracy and resolution. Current solutions include both approaches: WDM for temperature measurement and WDM + TDM for strain measurement. The main limitation regarding the number of sensors is the number of control lines, connectors and fibers that can be handled simultaneously without making the installation too complex and risky.

Permanent monitoring of deformations experienced by well tubular, casing and screens identifies the loads due to reservoir compaction or changes in the geological conditions, e.g. due to a squeezing salt layer as experienced by wells completed in pre-salt reservoirs. Strain measurements allow early prediction of catastrophic failure situations. Corrective action may then be taken to avoid the complete loss of the well. The axial strain, the radius of curvature of bending and crushing may be measured and, after signal processing, identification of the deformation mode: axial compressing, bending, ovalization, shearing, pressuring and/or thermal expansion can be computed (Rambow, Dria et al. 2010). This system is currently in the field trial stage.

Data for both types of quasi-distributed sensors (temperature and strain plus temperature) are shown in Table 3.2.

\section{2 .2}

\section{Single Phase and Two-Phase Flowmeter}

A fullbore, permanent, downhole flowmeter must operate over a wide range of pressures, temperatures, mixtures of fluids and flow regimes. Traditional multiphase flowmeters are not suited for this environment. Only one downhole flowmeter, with limitations related to minimum velocity and maximum acoustic noise, is currently being marketed. This flowmeter simultaneously measures the volumetric flow rate and the fluid's speed of sound using passive SONAR (Sound 
Navigation and Ranging) technology (Gysling and Loose 2003). The time averaged flow profile in turbulent flow is measured by each element of an array of sensors. This value is then used to identify and track spatially coherent structures (eddies) that are travelling with the flow. The signals from each element of the array are processed to deconvolve its frequency (f) and the wavelength (or length scale component, $\lambda$ ). The convection velocity can be then determined from the signal's spectrum by Equation 3.1:

$$
U=\frac{\omega}{k}
$$

Where

$$
\begin{array}{cl}
U & \text { - Convection velocity of the eddies; } \\
k=\frac{2 \pi}{\lambda} & \text { - Length scale of the structures (wavenumber); } \\
f=\frac{\omega}{2 \pi} & \text { - Frequency of the signal. }
\end{array}
$$

The volumetric flow rate is calculated from this velocity after a calibration procedure. Figure 3.2 shows a plot of radian frequency $(\omega)$ against wavenumber $(\mathrm{K})$. The dotted line in this plot corresponds to the convection (or bulk) velocity of the mixture.

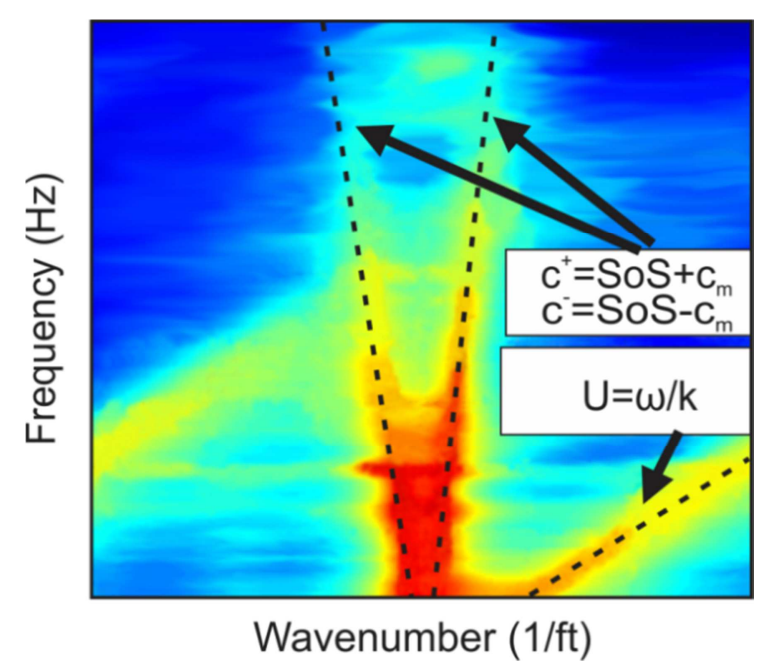

Figure 3.2 - Convection velocity and SoS (Speed of Sound) in k- $\omega$ plane.

The acoustic waves travelling in the fluid are also measured. They propagate both with and against the flow direction as a one dimensional acoustic wave (Figure 3.2), representing the same coherent structures convecting along the axis 
of the tubing. The velocity of the mixture and the SoS (Speed of Sound) can be determined from those velocities, see Eq. 2 and Eq. 3 (Unalmis, Johansen et al. 2010):

$$
\begin{gathered}
c_{m}=\frac{c^{+}-c^{-}}{2} \\
S o S=\frac{c^{+}-c^{-}}{2}
\end{gathered}
$$

It should be noted that these waves only propagate as a one dimensional wave if the frequency associated with the flow is beneath of a cut-off value: $f=\frac{1.84 S o S}{\pi D}$.

The volumetric fractions for single phase or liquid mixture are obtained from Eq. 4:

$$
c_{m}=y c_{l 1}+(1-y) c_{l 2}
$$

Where

$y$ - water fraction or water cut;

$c_{l 1}, c_{l 2}$ - constants obtained from the calibration procedure.

The speed of sound is proportional to the square root of the ratio of the compressibility and the density for multiphase flow. The mixture compressibility and density follow a similar relationship in which they are equal to the volumetrically averaged properties of the individual components (Eq. 5 and Eq. $6)$ :

$$
\begin{aligned}
& \rho_{m}=x \rho_{g}+(1-x) \rho_{l} \\
& \frac{1}{p_{m} c_{m}}=\frac{x}{p_{g} c_{g}^{2}}-\frac{1-x}{p_{l} c_{l}^{2}}
\end{aligned}
$$

Where

$\boldsymbol{x}$ - represents the gas volume fraction;

$\mathrm{c}_{l}, c_{g}$ - constants obtained from the calibration procedure.

This technology actually measures the dynamic strain in the tubing caused by pressure fluctuation due to the turbulent flow. This passive technique is only suitable for relatively high rate wells. 
The acoustic noise generated by the IW valve can also be used to confirm the valve's opening positions. The majority of multi-position valves currently used do not have position sensors with the valve position being derived from previous information on valve movements stored in the control unit. Operational experience has shown that it is not unusual for knowledge of the valve position to be uncertain. It can only be confirmed by closing the valve completely, with consequent loss of production.

Other diagnostics could be added to improve the condition monitoring of downhole equipment. As far as we are aware this is not even being trialed.

Typical sensor data is shown in Table 3.2.

\subsection{3}

\section{Permanent Downhole Seismic Sensors}

The first fiber optic seismic sensors for permanent downhole measurements were installed in southwestern France for a walkway VSP in 2002 (Knudsen, Havsgard et al. 2003). It was a six level, tri-component (3C) accelerometer system used for monitoring a gas storage field. Neither electronic nor fiber optic permanent downhole seismic sensors have been trialed for installation in an IW to-date. The advantage of the fiber optic accelerometers is their improved metrological data (dynamic range, distortion, noise floor and bandwidth). In addition, fiber optic accelerometers bring operational advantages for OBC seismic (no batteries or heavy copper cables). The result is high resolution seismic imaging and better dynamic reservoir characterization (4D) when one adds up seismic acquisition reproducibility, acquisition frequency flexibility and improved metrological parameters of the sensors. Typical data for downhole seismic sensors are shown in Table 3.2. Table 3.1 is a simple comparison of the three seismic sensor technologies available. This table illustrates the important differences regarding noise and bandwidth between the two new technologies which result in improvements in noise of seismic deeper traces and in trace resolution.

\begin{tabular}{llll} 
& \multicolumn{1}{c}{ Electromagnetic } & \multicolumn{1}{c}{ MEMS } & \multicolumn{1}{c}{ Fiber Optic } \\
\hline Noise floor & $<1000 \mathrm{ng} / \sqrt{\mathrm{Hz}}$ & $<700 \mathrm{ng} / \sqrt{\mathrm{Hz}}$ & $<100 \mathrm{ng} / \sqrt{\mathrm{Hz}}$ \\
Bandwith & $10-240 \mathrm{~Hz}$ & $0-800 \mathrm{~Hz}$ & $1-1.4 \mathrm{kHz}$ \\
Dynamic range & $90 \mathrm{~dB}$ & $120 \mathrm{~dB}$ & $130 \mathrm{~dB}$ \\
Distortion & $-70 \mathrm{~dB}$ & $<-90 \mathrm{~dB}$ & $<-90 \mathrm{~dB}$
\end{tabular}

Table 3.1 - Seismic sensors comparison by technology. 


\subsection{4 \\ Distributed Temperature and Distributed Temperature and Strain Sensors}

Distributed downhole sensors gave the missing spatial component to the reservoir and production engineers. The first distributed sensor for downhole applications was based on Raman backscattering, the DTS. It is also available today DTSS (Distributed Temperature and Strain Sensing) based on Brillouin backscattering. Both methods are based on changes in the spectral content as a result of nonlinear interactions of the light propagating through the fiber optic. It uses the optical domain reflectometry technique which sends a series of optical pulses into the fiber and extracts the scattered light from the same end of the fiber (Figure 3.3). Raman backscattering measures the intensities of the Raman signal at equal energy differences in opposite shifted directions, producing a ratio which depends only on temperature (Culshaw and Kersey 2008). The backscattered light is coupled, due to stimulated Brillouin backscattering, with an acoustic wave which has half of the wavelength of the incoming light. The measured frequency shift is the frequency of the acoustic wave which depends on the density ratio and, therefore, on the temperature and strain (Culshaw and Kersey 2008).

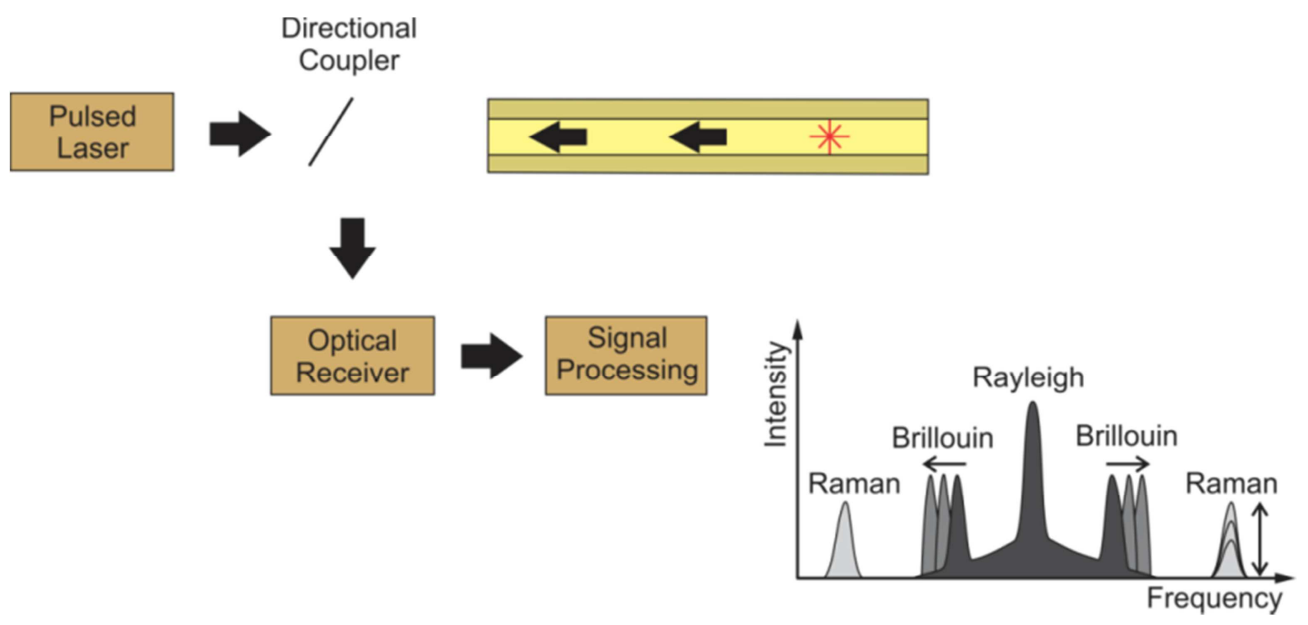

Figure 3.3 - Backscattering based sensors.

DTS is widely used for steam injection/production profile monitoring and for flow allocation in wells with dry CT. Metrological and operational restrictions have resulted in it not being fully adopted by operators for deepwater, subsea installations. This is partly because the metrological parameters for a distributed fiber optic measurement are not straightforward. The specification of distributed systems depends on all the parameters which affect the measurement, e.g. total length (distance), acquisition rate, spatial resolution and fiber attenuation. Hence, 
one has to adjust the spatial and time resolution to meet the specifications of the system being designed using a chart which plots the data as a function of the above parameters. Figure 3.4 is such a performance chart for the hypothetical system. It illustrates that a distributed measurement system based on optical domain reflectometry needs more than one parameter, the distance and sample rate for this system, to define its metrology performance. Note that the system resolution is limited by its repeatability.

DTS has been used with IWs, but not DTSS. The value-added by first recognizing and then identifying the cause of well integrity issues may prove to be a justification for this type of monitoring system. They should be used when there is a high risk of reservoir compaction, formation movement or high corrosive environment.

Typical DTS and DTSS data are shown in Table 3.2.

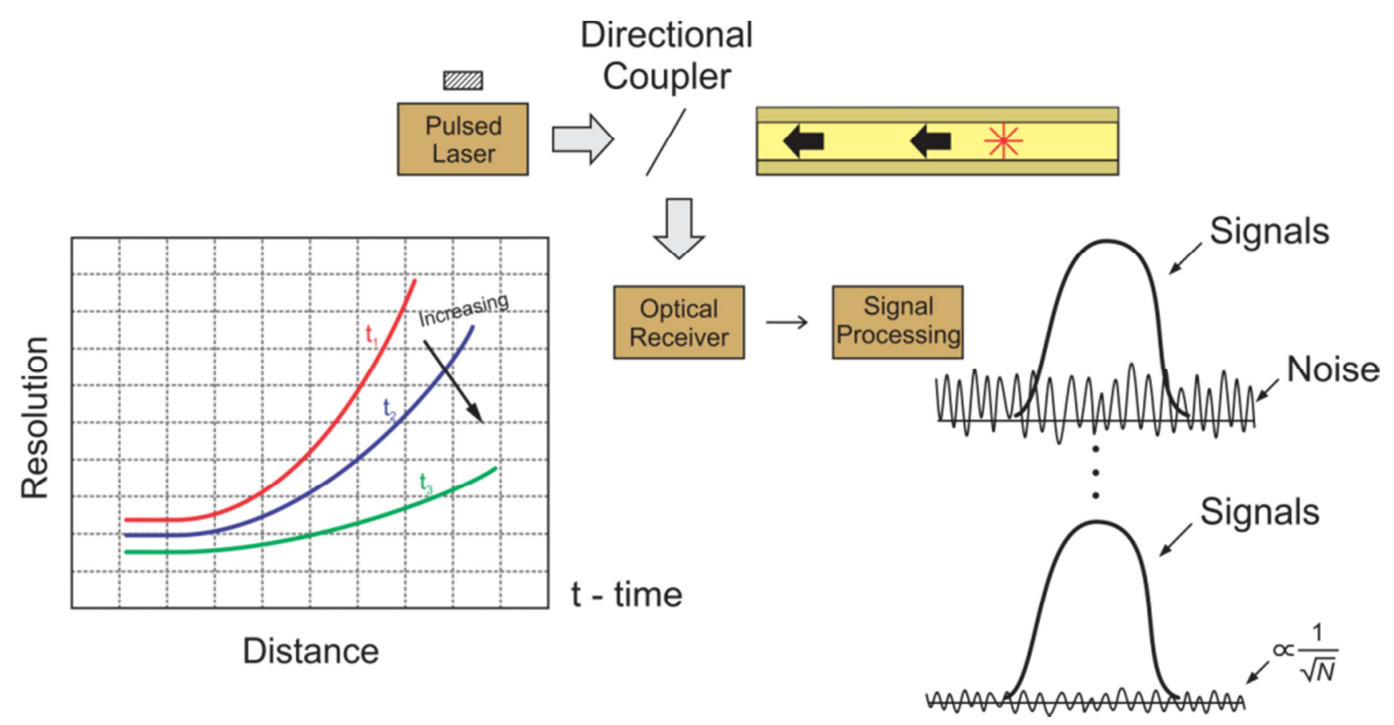

Figure 3.4 - Performance chart

\subsection{5}

\section{Distributed Acoustic/Vibration Sensors}

Acoustic signals are widely measured \& interpreted in upstream Oil and Gas production activities. They are used for condition monitoring, failure prediction and diagnosis of the cause of failure in rotating machinery. They have become an important component of the predictive maintenance strategy. In addition, acoustic signals can be correlated to flow rate, the position of a downhole valve, the correct functioning of a gas lift valve, etc. 
The combination of a DAS/DVS (Distributed Acoustic Sensing/Distributed Vibration Sensing) used in conjunction with distributed temperature and single point pressure and temperature sensors could provide a wealth of information about the downhole flow conditions as well as the performance of the equipment and production process. Such combinations of downhole measurements have only recently become available at the same operational complexity as DTS where the ability to share the same (preferable single mode) optical fiber is an advantage. This makes DAS/DVS a real option for deepwater environment where only one pin fiber optic tubing hanger connector is in the advanced stage of development.

This technology was first trialed in a tight gas well to optimize the design and execution of a hydraulic fracturing operation (Molenaar, Hill et al. 2011). Figures. 3.5 and 3.7 illustrates two DAS/DVS results from this paper. Figure 3.5 is the trace generated by a bridge plug being set while Figure 3.7 shows measurements during a hydraulic fracturing treatment. The most effective treatment, as indicated by an intense red signal, is at the toe of the well. IW applications have not been reported to date but quantitative flow through the valve and confirmation of the choke position are potential ones.

In addition, development of distributed flow metering based on SONAR principles might be possible. Figure 3.6 shows another possible application: flow allocation. Although there are qualitative techniques available, this type of sensor has a high potential. This technique might also be useful for geophysical monitoring applications. A reduced risk level in addition to a simpler operation was observed during VSP field trials. A low signal to noise ratio was observed though (Mestayer, Cox et al. 2011).

The technology is based on coherent Rayleigh backscattering using a highly coherent laser and a time gated interferometer to measure the local acoustic and vibrational disturbances. These disturbances induce a small strain on the fiber which causes a change in the path of the Rayleigh scattering. Exciting the fiber with pulses of highly coherent laser light causes optical interference between light scattered by different paths within the length of the pulse. The backscattered signal is then phase demodulated at a specific time delay to recover the acoustic and vibrational disturbances acting at discrete points along the fiber. 


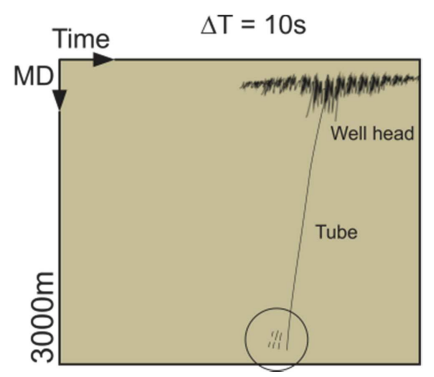

Figure 3.5 - Packer setting confirmation.

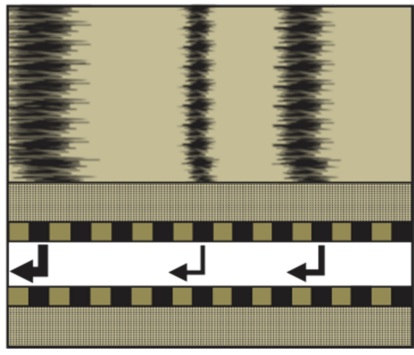

Figure 3.6 - Flow allocation analysis.

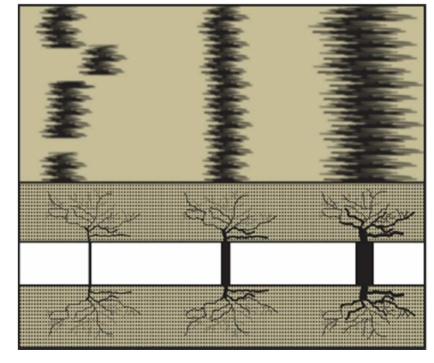

Figure 3.7 - Hydraulic fracturing performance monitoring.

Techniques based on polarimetry are also used in distributed sensing. Changes in local birefringence are measured in the context of polarimetry through optical time domain reflectometry forming the basic principle of the distributed pressure sensing system which is in the early stage of development for downhole applications.

\section{3 \\ Metrology and Reliability}

When making a comparative analysis of sensors for measuring physical quantities such as pressure, temperature and flow it is important that we use a universal language. These are defined by the competent bodies such as the NIST (National Institute of Standards and Technology) in the U.S.A. Such language eases communication between purchasers, suppliers and manufacturers by preventing (unfortunately common) specification errors. The harsh subsurface environment coupled with the high cost of maintenance adds an additional importance to understanding the metrological parameters and their reliability. Some important definitions from the International Vocabulary of Basic and General Terms in Metrology - VIM (2007) (ISO/IEC 2007) are listed below and in Figure 3.8:

- Accuracy - Close agreement between a measurement result and the measurand true value. Indicates the distance between the absolute value of a physical quantity and its standard controlled by the competent bodies. It is related to the systematic errors of the instrument. 
- Repeatability - Close agreement between the results of successive measurements of the same measurand made by the same measurement conditions. It is expressed as multiples of standard deviation.

- Reproducibility - Close agreement between the results of successive measurements of the same measurand made by variable measurement conditions. The reproducibility indicates the repeatability in all points of the calibration range.

- Resolution - The smallest difference that can be seen between the instrument indications. It is limited bellow by the repeatability.

- Stability - Ability of an instrument to maintain its metrological characteristics over a specified period of time. It is usually evaluated over both short and long periods of time. The stability measurement over a short period of time is also known as repeatability. The slow variation in the metrological characteristics of a measurement instrument known is called drift. The drift is one of the components of the instrument longterm stability.

- Measurement uncertainty - Indicates the measurement dispersion around its true value of the instrument's reliable operational range. The factors that affect the measurement uncertainty are classified as either type A or type B. Components factors that are evaluated from a statistical distribution of observations, and are hence characterized by a standard deviation, are defined as being of the type A. Type B factors are evaluated from probability density functions based on experience or other means not associated to observations.

The measurement uncertainty can be evaluated as standard, combined or extended. The standard measurement uncertainty is estimated by a standard deviation (or a multiple of it). The combined uncertainty reflects the influence of the individual components that make up the standard uncertainty. The extended uncertainty is the combined uncertainty multiplied by a confidence interval. Measurement uncertainty should be evaluated following the ISO-Guide98-3: Guide to the Expression of Uncertainty in Measurement (ISO/IEC 2008). 


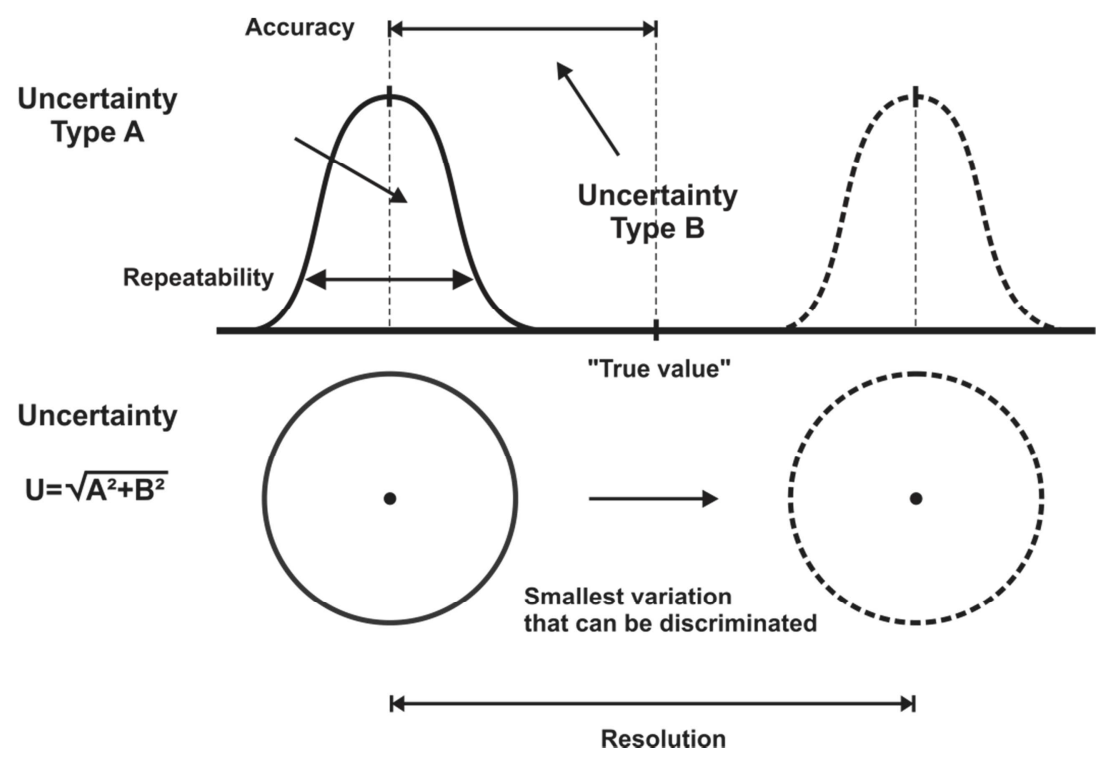

Figure 3.8 - Metrological parameters definition and relationship.

The comparison between downhole sensors is normally a difficult task. Military standards are available for comparing sensors with respect to reliability. Unfortunately such standards are not available for metrology. Hence both operators and suppliers have been forced to create their own standards for metrological aspects based on experience and field proven parameters. The situation is even worse for new technologies, such as fiber optic sensing. The lack of suitable standards, qualification tests and quantitative performance standards hindered the introduction of the technology into the field. In fact, it was recognized that their development was essential for the technology adoption.

The biggest metrological problem associated with downhole sensors is inability to periodic calibration. This disability indicates the need for equipment with a high level of long-term stability together with the use of measurement uncertainty propagation algorithms in the models and optimization tools used to control the field production.

\subsection{1}

\section{Necessary Measurement Uncertainty and Placement Optimization}

Total model uncertainty is typically studied by the quantifiable uncertainties present in the studied system. The real uncertainty may, on the other hand, be larger due to the lack of knowledge. Sources of quantifiable uncertainties can be summarized as input, parameter and structure uncertainties and we restrict our analysis to them. Monitoring systems impacts directly input and parameters 
uncertainties through direct measurement and model calibration. Structure uncertainty normally is forgotten when it comes to the metrological specification of monitoring systems. The total model uncertainty is changed by the structure uncertainty which can be seen as an independent source of uncertainty due to model equations, boundary conditions, simplifications, resolution, time and space extrapolation, numerical errors, etc. (Figure 3.9). Therefore it should be taken into consideration for an appropriate assessment of the input and parameters uncertainty.
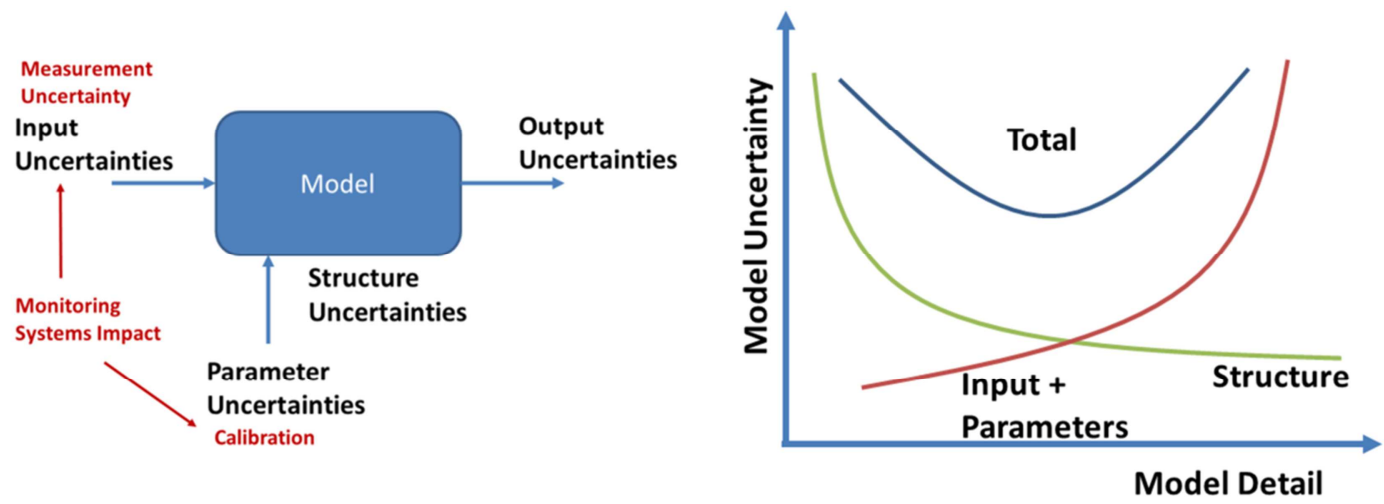

Figure 3.9 - Quantifiable source of model uncertainties and their impact.

A monitoring system must be designed to measure important events with a given uncertainty $\left(\delta_{E}\right)$. Estimation of the necessary measurement uncertainty $\left(\delta_{M}\right)$ depends on the data interpretation method employed and its relative uncertainty $\left(\delta_{I}\right)$. Generally speaking, if a measurement is related to an event and additional measurements $M^{*}\left(M=f\left(E, M^{*}\right)\right)$; then the total measurement uncertainty or minimum resolution can be calculated as (Silva, Muradov et al. 2012):

$$
\delta_{M} \leq \sqrt{f_{I}\left(E, M^{*}\right)^{2} \delta_{I}^{2}+\sum_{E, M^{*}}\left[\left(\frac{\partial f}{\partial\left(E, M^{*}\right)}\right)^{2} \delta_{E, M^{*}}^{2}\right]}
$$

Where

$f_{I}\left(E, M^{*}\right)^{2} \delta_{I}^{2}$ is the event interpretation model uncertainty;

$$
\sum_{E, M^{*}}\left[\left(\frac{\partial f}{\partial\left(E, M^{*}\right)}\right)^{2} \delta_{E, M^{*}}^{2}\right] \text { is the uncertainty from the measurements and }
$$

event propagated throughout the model or from a sensitivity analysis; 
$M^{*}$ is the additional measurement;

$E$ is the event.

There are numerous examples of such measurement-to-event relationships in the literature. For example the distributed temperature can be related to the single-phase flow rate via the classical equation (Ramey Jr. 1962; Elshahawi, Osman et al. 1999):

$$
\frac{T_{e}-T_{w f}}{d T_{w f} / d z} \approx q \frac{\rho C_{p}\left(K+f(t) R_{w b} U\right)}{2 \pi K R_{w b} U}
$$

Another example is the relation between the measured acoustic noise level (>600 Hz) $\mathrm{N}^{*} 600$ and the single-phase flowrate $q$ and cross-sectional area $A$ (McKinley and Bower 1979):

$$
N_{600}^{*}=C \frac{\rho q^{3}}{A^{2}}
$$

Note that the relative uncertainty of this method is relatively low $\left(\delta_{I} \sim 0.2\right)$.

The time resolution required to reliably identify the desired event depends on the event characteristic time scale. A good selection of characteristic time scales is provided by (Nyhavn, Vassenden et al. 2000).

Optimizing the placement of a single point sensor can be done by the use of Experimental Design. The wellbore and reservoir are modeled for different flow conditions to provide (noisy) input data for back-calculation of phase flow rates with the gauges placed at a number of locations. Multiple completion designs may be evaluated and ranked according to their confidence level. Examples are available from (Naevdal, Vefring et al. 2001).

\subsection{2}

\section{Reliability}

The reliability of a system or component is defined as its ability to perform a required task under the design conditions over a specified period of time (Rausand and Høyland 2004). It is generally presented as either a "probability of failure" or a "probability of availability". Long-term reliability was the first barrier to IW technology adoption. Even today, the design engineer must include the supplier's quality assurance and reliability management program when 
specifying equipment. This program should incorporate continuous design improvement, manufacturing and testing based on the observed reliability performance.

Accelerated life time testing as well as risk assessment evaluation for installation, operation and maintenance are also required (Aggrey 2007). For example, a three zone IW based on electric/hydraulic technology has a survival probability or reliability of better than $85 \%$ after 5 years.

API-17N deals with general project risk management in subsea operations while ISO 13628-6 provides a similar level of guidance for production control system and interface reliability.

A work from (Mathieson, Rogers et al. 2003) divided IW reliability into mission reliability (survival of the IW equipment) and system reliability (full completion survival). IW system reliability is further divided in four components: monitoring, flow control, subsea and integration. Reliability analysis of these components can be used to quantify the impact of reliability on the "Value Added" by the availability of monitoring information, zonal flow control and its right-time optimization. Initiatives regarding quantification of the IW added value taking into account reliability can be found in the literature (Ajayi, Mathieson et al. 2005; Aggrey, Davies et al. 2006; Aggrey 2007). These papers mainly focus on the valve failure due to the impact of being unable to choke (for multi-position applications). They also included the cost of an unplanned intervention (which may not happen due to cost). Note that the IW equipment from all major suppliers has a valve failure mode "as is". A change of its position to fully open or fully closed is possible via a coiled tubing intervention though. The quantification of reliability on the value of an IW monitoring system has been explored but not yet published.

The data from the IW monitoring system in an integrated framework can be shared by all production processes, minimizing costs and increasing the monitoring system attractiveness. The most important factors that lead to the selection of IW completions are the reduction in the number of (i) wells and (ii) risky interventions (due to reservoir control) and (iii) accessing marginal reserves (Hudson, Alves et al. 2011).

A typical IW monitoring system consists of more than one sensor and its mandrel, a dry connector (sensor to control line and control line to control line), 
an electrical control line, TH (Tubing Hanger)/WCT wet-mate feedthrough connector system, wellhead outlet, jumper, IWCS, umbilical and IWSCS. An extrapolated five year reliability of better than $90 \%$ is typical of the newer generation of electronic pressure and temperature sensors in a three zone IW application.

Oreda database is a good starting point for equipment reliability data for topside and subsea equipment. The availability of a reliability database which automatically updates from day-to-day operational events and the resulting, field validated, equipment performance statistics coupled to production applications will yield great value for operators. Note that the operations and maintenance information open standard (MIMOSA) should be used to exchange data among production applications (as has been proposed by PRODML).

\section{4 \\ Basic Integrated IW Monitoring System Design Framework}

Well completion design within an integrated modeling and optimization framework is a challenging task since today's technology has resulted in a wide variety of hardware and software being employed to manage the current well stock. A well completed with sensors and downhole flow control to achieve specified objective needs downhole information to allow well management. The required downhole information in most cases cannot be directly measured. It normally has to be derived from the parameters which can be measured with today's sensors. This can be illustrated by considering how a zonal flowrate might be measured by a downhole flowmeter together with all the auxiliary measurements and interpretation methods that are needed. This includes differential pressure measurement across a Venturi, distributed temperature, distributed acoustic, etc.

Downhole monitoring system is aimed to maximize the IW value with minimum cost. It might be considered as a main driver for the completion design in a very general sense. Figure 3.10 shows permanent downhole monitoring design basis for the integrated modeling and optimization framework. 


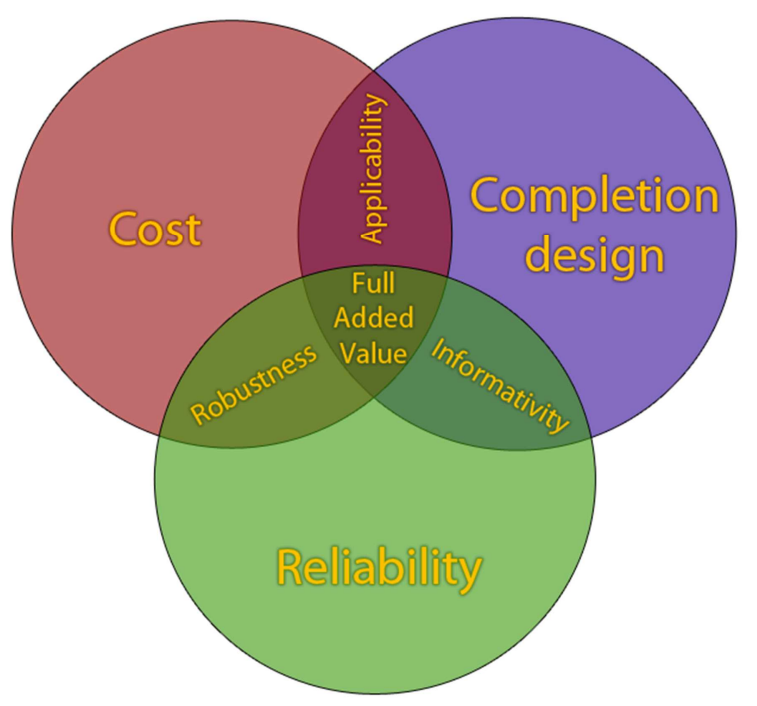

Figure 3.10 - IW monitoring system design basis

The following workflow illustrates an IW monitoring system design process:

1. Identify where an IW is expected to add value and what information is needed for an effective decision making.

2. Identify the major IW risks expected throughout its lifetime. The risks might include well completion conditions, zonal performance changes, installation complexity, equipment failure, etc. Subsequent well treatment/workover operations needed to these effects should be also evaluated.

3. Identify sets of available sensors that fit the above objectives, its reliability, cost and available interpretation methods.

4. Screen and rank these sets in the terms of robustness, applicability and informativity.

This basic workflow will help find an optimal downhole monitoring system aimed at achieving the full added value. Note that the use of the word "informativity" above refers not only to the values of the parameters being measured, but also to their spatial and temporal resolution.

This is a very broad area that is impossible to cover in detail. We will attempt to do a brief description of the sensor application areas followed by a conceptual design example. 


\section{5}

\section{Application Areas}

A summary including measurement relevance to different application areas is presented in Table 3.3. Note that instead of sensors there are measurements to the application areas relation due to the fact that a combination of different sensors can provide similar sets of measurements. As always, some of the standalone measurements are impossible to detect certain events, measurement combinations however help. The application areas have been grouped into:

- Conditional monitoring;

- Well performance;

- Well stimulation;

- Flow assurance;

- Advanced completions;

- Reservoir characterization.

Published studies which have a similar intent of this chapter are commented below:

- (Nyhavn, Vassenden et al. 2000), who provided a very good insight on what information is needed for effective IW control, ranked the available measurements and sensors according to their availability (at that time), reliability, and simplicity to the application area. They have analyzed the major problems of IW management - complex and computationally demanding model based IW control, associated information needed, measurement and event time scales and subsequently large data storage, and processing and analysis power needed. Introduction of the advanced data processing methods - multivariate analysis, time-lapse processing and softsensing - were viewed to be of particular importance in the years to come. Recognition that much IW value derives from the ability to "handle surprises" and promptly take action based on the high quality information from the downhole monitoring systems has been justified over the last decade.

- Discussion on the available monitoring systems, the missing monitoring areas and the monitoring equipment evolution from the point of view of IW management was presented by (Kluth, Varnham et al. 2000). They have also 
provided an example of how the (monetary) value of information can be estimated.

- An attempt to overview major monitoring system design approaches based on the above publications, including uncertainty and reliability considerations, along with a list of available sensors and their applications was provided by (Pari, Kabir et al. 2009).

\section{6 \\ IW Monitoring System Design Example}

Let us consider a synthetic abstract example based on the PUNQS3 reservoir model; a simplified version of a real reservoir that is publically available. It has previously been shown by (Birchenko, Demyanov et al. 2008; Grebenkin and Davies 2010) to be a suitable candidate for IW. We will use the optimal production strategy as developed by these authors. It consists of one, horizontal, 4 zone IW producer and two vertical injection wells to support reservoir pressure and dispose the produced gas. Figure 3.11 (middle) shows the reservoir top view, the horizontal IW production well and the two injectors. The production limits are liquid rate $\leq 600 \mathrm{sm}^{3} / \mathrm{d}$ and bottom hole pressure $>120$ bar.

A reactive strategy (after breakthrough) is planned for controlling the IW, the ICVs are operated based on zonal WC (Water Cut) and GOR (Gas-Oil Ratio) (Grebenkin and Davies 2010):

$$
a^{i}=\frac{Q_{l i q}^{i}}{Q_{o i l}^{i}}=\frac{B_{o}+\left(G O R^{i}-R s\right) B_{g}}{1-B_{w} W C^{i}}
$$

The optimization strategy uses the normalized value $a^{i}$ as the choke criterion for each ICV trying to improve oil production while keeping the same liquid rate. The ICV of the zone $i$ is closed (or choked) iteratively starting always from the zone with highest WC. The control decision made is confirmed if an oil production improvement is achieved at each time step. A commercial reservoir simulator (Eclipse 100) with multi-segment capability was used in the simulations resulting an additional oil production of $0.17 \mathrm{MMsm3}$ (i.e. about $\$ 64$ million assuming oil price of $\$ 60 / \mathrm{bbl}$.) for the worst case scenario or reservoir uncertainty 
(Grebenkin and Davies 2010). All associated IW completion and operation costs should be compared against this additional revenue.
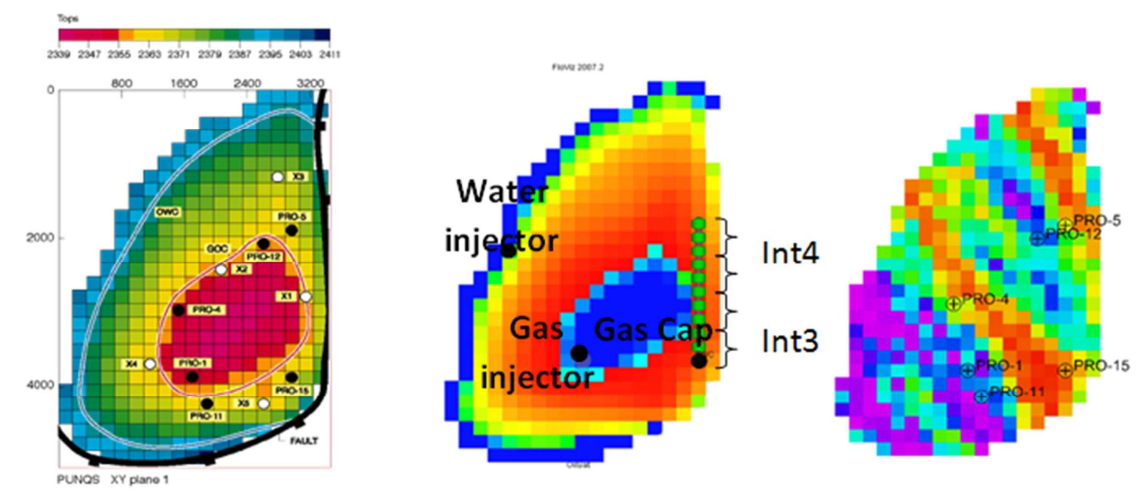

Figure 3.11 - Structure (left), well locations (middle) and permeability distribution (right) for the PUNQS3 model (Grebenkin and Davies 2010).

The horizontal IW producer contacted four reservoir intervals over its completion length of $1800 \mathrm{~m}$. It had to deal with uncertainty in the distribution of both the static and dynamic reservoir properties as well as in the vertical or lateral connectivity. Effective control of the IW requires information at the zonal level to optimize the production process. A constant liquid production rate with changing reservoir and production conditions requires an artificial lift system which reacts to changes in the BHP and phase cut. Gas lift was chosen as the artificial lift method because of these conditions and the gas cap. Additional simulations suggest that the ICVs will require changing once per month to get full additional production.

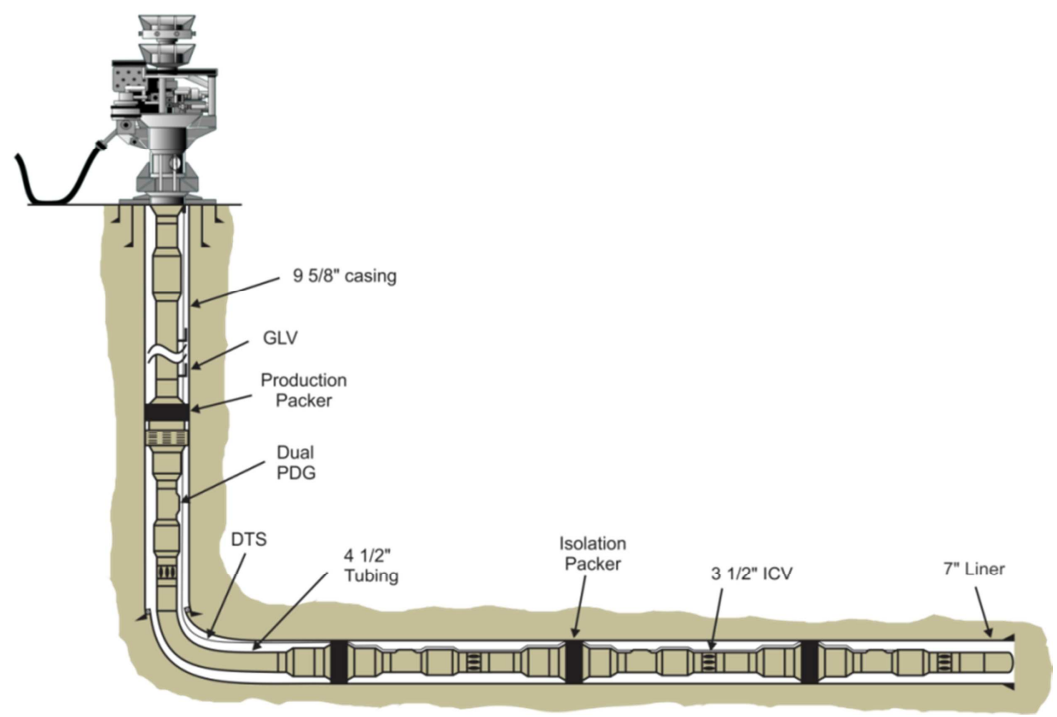

Figure 3.12 - Horizontal IW completion for PUNQ S-3 production well. 
The well design consisted of a $95 / 8$ " casing above the reservoir with a 7" liner installed across the $8 / 12$ " openhole reservoir section with $4 \frac{1 / 2}{2}$ " production tubing, isolation packers, 3 1/2" multi-position inflow control valves and sensors (Figure 3.12). The total depth of the well is $4500 \mathrm{~m}$ with GLV (Gas Lift Valve) located at $1560 \mathrm{~m}$ (all measured depths). This is not an optimal design for high rate wells or long horizontal wells due to maximum rate restriction but this is not the case.

The reservoir data and well design suggest that the monitoring system should be able to monitor zonal WC, zonal pressure and the performance of the gas lift system. The required resolution of the zonal WC measurement was identified as being the smallest change in WC that would lead to a control decision being made by the equation 3.10. The optimal oil production for the 4 zones are: $80.6,141.1,15$ and $100 \mathrm{sm}^{3} / \mathrm{d}$ after valve 1 and 4 being choked which corresponds to water cut changes of: $-2,5,10$ and $-10 \%$ respectively and $4.6 \%$ of improvement in oil production. They were found using the WC optimization strategy on commercial reservoir flow simulation (Eclipse $\left.{ }^{\mathrm{TM}} 100\right)$. Figure 3.13 shows the simulation results (marked lines) and the zonal WC values (solid lines). The smallest change in WC between two control actions occurred for Zone 1 in 1976. It corresponds to $2 \%$ of the absolute value which would need to be measured at zonal liquid rates ranging from 50 to $250 \mathrm{sm}^{3} / \mathrm{d}$.

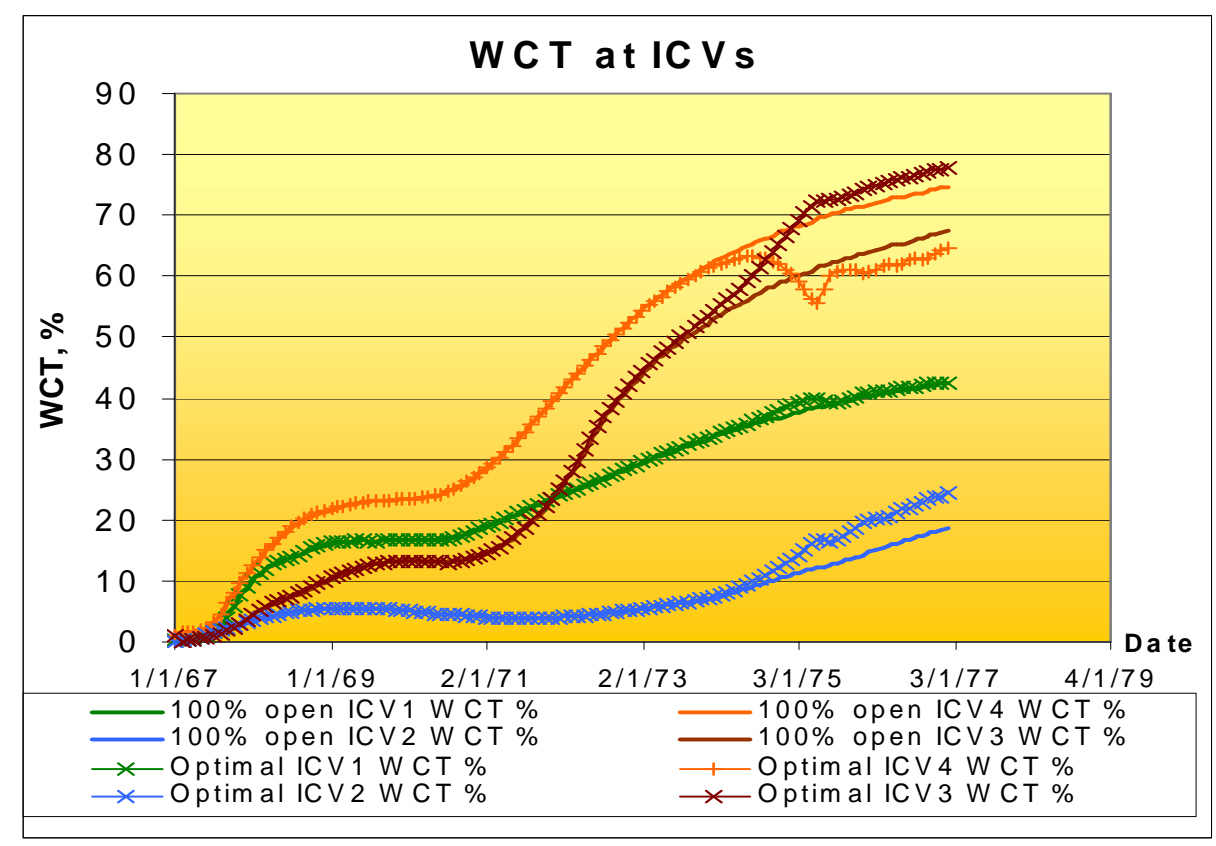

Figure 3.13 - WC simulation results. 
Measurement of WC might be available by:

- Soft-sensing using single-point pressure and temperature sensors of each zone (Leskens, Smeulers et al. 2008);

- Single-point pressure and temperature of each zone, a differential pressure based flowmeter, and a densitometer;

- $\quad$ Surface MPFM (Multi-Phase Flowmeter).

The first alternative uses a soft-sensing algorithm which depends on an integrated production model (reservoir, well, chokes, and pipeline models) and an error minimization procedure between calculated and measured data. It has at least $10 \%$ of model uncertainty (depending on the commercial software used) plus less than $1 \%$ of measurement uncertainty which using equation 3.7 results in $10.05 \%$ of total uncertainty. Analyzing the second alternative, the most important decision is the appropriate choice of the flowmeter. The low zonal flow rate implies that SONAR or Inverted Venturi based flowmeter is not an option due to their minimum flow rate limits. An insertable Venturi based flowmeter could be used but it has a diameter restriction as a drawback which leads to a time consuming and risky operation every time an intervention is needed below the flowmeter. The uncertainty in this case is $2.5 \%$ due only to the measurement uncertainty. The third alternative is a surface MPFM which has typically $5 \%$ of WC measurement uncertainty for GVF (Gas Void Fraction) lower than 60\% which is the case for this example.

The alternatives for the zonal pressure and gas lift performance are:

- PDG for each zone plus DTS or

- PDG for each zone plus quasi-distributed temperature.

The PDG should have a dual-gauge configuration (annulus and tubing measurement) allowing formation and production test to be carried out in addition to average reservoir pressure. Measurement uncertainty of quartz based PDG is enough for both applications. Continuous gas lift control is done by changing the average oil density above the gas lift valve (gas injection point) increasing the drawdown $\left(P_{r}-P_{w f}\right)$. The objective is maximize $f\left(q_{i}, q_{p}\right)$ constrained by $q_{i m a x}$ and $q_{p \min } \leq q_{p} \leq q_{\text {pmax }}$ where $q_{p}=q_{p}\left(q_{i}\right)$. The gas lift performance is impacted by the reactive control strategy adopted which changes the WC. Neglecting friction and kinetic energy $P_{w f}$ can be written as: 


$$
p_{w f}=p_{w h}+h_{i n j}\left(\frac{\partial p}{\partial z}\right)_{1}+\left(h-h_{i n j}\right)\left(\frac{\partial p}{\partial z}\right)_{2}
$$

Where

$$
\left(\frac{\partial p}{\partial z}\right)_{i}=\rho_{i}(p, T) g \quad, i=1,2 .
$$

Pressure and temperature measurement should be used for tuning the operational point when WC changes due to the ICV management. A temperature simulation using a commercial dynamic simulator $\left(\mathrm{Olga}^{\mathrm{TM}}\right)$ for the base case (all ICVs producing) and the worst case (only two ICVs producing) is shown in Figure 3.14 after a stabilization time of one hour. It is possible to see qualitative results of the reactive control strategy and an appropriate identification of the GLV injection point. The temperature resolution should be no less than $1.5 \mathrm{C}$ according to this sensitivity study (note the temperature changes at GLV depth of 1560 m MD - Measured Depth). In addition, when only two zones are producing, the temperature is lower than the one when all zones are producing for the same total flowrate, meaning that more oil is being produced as oil specific heat at constant pressure is lower than the water one. The use of DTS requires $8 \mathrm{~m}$ of spatial resolution to allow recognition of the temperature decline at a working GLV. Note that a typical DTS system achieves this resolution in about 30 minutes of temporal averaging while having a nominal spatial resolution of $2 \mathrm{~m}$ assuming a total length of $6 \mathrm{~km}$.

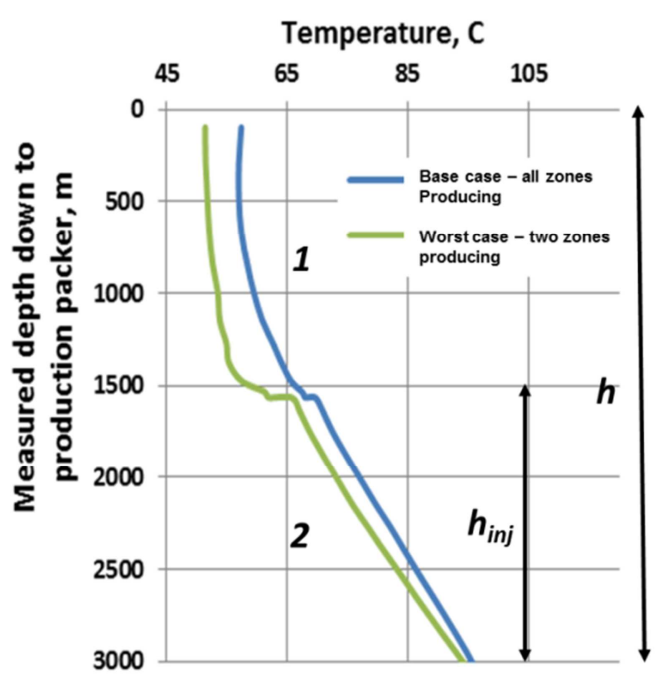

Figure 3.14 - Temperature in lower completion showing two and all valves open. 
Analyzing the resulting monitoring system alternatives, there is no monitoring system alternative that matches the target $2 \%$ of WC uncertainty required. A study of the impact on the control strategy should be done to identify if there is a loss in the incremental IW benefit if a higher uncertainty is used. Zonal pressure and gas lift performance monitoring can be done using PDG and DTS (or quasi-distributed temperature sensing in the case of fiber optic cannot be installed) without special requirements as previously discussed.

The Integrated IW monitoring system for this example which minimizes equation 3.7 is a dual configuration PDG installed in each zone, a single mode fiber for DTS, and a surface MPFM. There are no major issues with the reliability of the IW monitoring system chosen and there are enough data available to perform reliability assessments studies. Additionally, if a DAS is installed will bring more reliable results with new diagnostic possibilities (ICV position confirmation, sand production monitoring, etc.). 


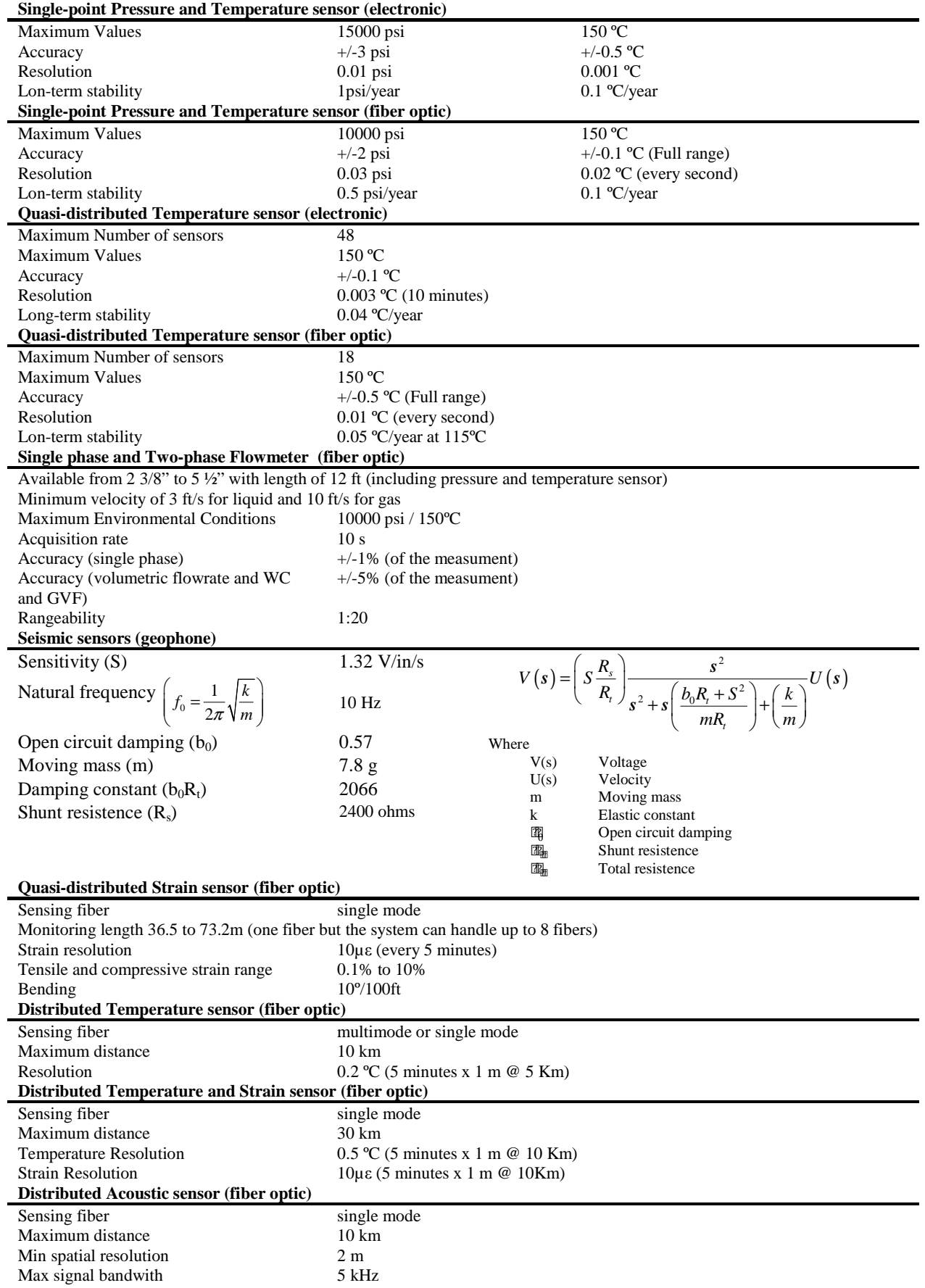

Table 3.2 - Typical metrological data for downhole sensors 


\begin{tabular}{|c|c|c|c|c|c|c|c|c|c|c|c|c|}
\hline \multirow{2}{*}{ Type } & \multirow{2}{*}{ Application } & \multicolumn{4}{|c|}{ Distributed } & \multicolumn{7}{|c|}{$\begin{array}{c}\text { Quasi-distributed/Discrete (limitations might be } \\
\text { applied due to spatial resolution) }\end{array}$} \\
\hline & & $\mathbf{T}$ & A & v & $\varepsilon$ & $\mathbf{T}$ & $\mathbf{P}$ & A & $\mathrm{s}$ & $\mathbf{Q}$ & $\varepsilon$ & EM \\
\hline \multirow{3}{*}{ 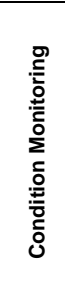 } & $\begin{array}{l}\text { Artificial lift } \\
\text { (operating GLV, ESP } \\
\text { performance, etc.) }\end{array}$ & $\sqrt{ }$ & $\sqrt{ }$ & $\sqrt{ }$ & & $\sqrt{ }$ & $\sqrt{ }$ & & & & & \\
\hline & $\begin{array}{l}\text { Well/Pipeline Integrity } \\
\text { (casing leak, flow behind } \\
\text { casing, packer isolation, } \\
\text { etc.) }\end{array}$ & $\sqrt{ }$ & $\sqrt{ }$ & $\sqrt{ }$ & $\sqrt{ }$ & & & & & & $\sqrt{ }$ & \\
\hline & $\begin{array}{l}\text { Well/Pipeline Structural } \\
\text { integrity (reservoir } \\
\text { compaction, formation } \\
\text { movement, corrosion, } \\
\text { etc) }\end{array}$ & & & & $\sqrt{ }$ & & & & & & $\sqrt{ }$ & \\
\hline \multirow{6}{*}{ 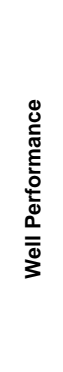 } & $\begin{array}{l}\text { Injection or production } \\
\text { flow rate profiling }\end{array}$ & $\sqrt{ }$ & $\sqrt{ }$ & & & $\sqrt{ }$ & $\sqrt{ }$ & & & $\sqrt{ }$ & & $\sqrt{ }$ \\
\hline & Influx identification & $\sqrt{ }$ & $\sqrt{ }$ & & & $\sqrt{ }$ & $\sqrt{ }$ & $\sqrt{ }$ & & $\sqrt{ }$ & & $\sqrt{ }$ \\
\hline & Sand production & & $\sqrt{ }$ & $\sqrt{ }$ & & & & $\sqrt{ }$ & & & & \\
\hline & Gas/water/oil cut profiling & $\sqrt{ }$ & $\sqrt{ }$ & & & $\sqrt{ }$ & $\sqrt{ }$ & $\sqrt{ }$ & & $\sqrt{ }$ & & $\sqrt{ }$ \\
\hline & $\begin{array}{l}\text { Cross-flow between } \\
\text { zones/layers }\end{array}$ & $\sqrt{ }$ & $\sqrt{ }$ & & & & & & & & & \\
\hline & $\begin{array}{l}\text { Producing zone/layer } \\
\text { identification }\end{array}$ & $\sqrt{ }$ & $\sqrt{ }$ & $\sqrt{ }$ & & $\sqrt{ }$ & & $\sqrt{ }$ & & & & \\
\hline \multirow{4}{*}{ 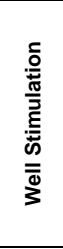 } & $\begin{array}{l}\text { Perforated intervals } \\
\text { identification }\end{array}$ & $\sqrt{ }$ & $\sqrt{ }$ & $\sqrt{ }$ & & & & & & & & \\
\hline & $\begin{array}{l}\text { Acidized intervals } \\
\text { identification }\end{array}$ & $\sqrt{ }$ & & & & & & & & & & \\
\hline & Well cleanup & $\sqrt{ }$ & & & & $\sqrt{ }$ & $\sqrt{ }$ & & & & & \\
\hline & $\begin{array}{l}\text { Hydraulic fracture } \\
\text { (height/length/location } \\
\text { identification) }\end{array}$ & $\sqrt{ }$ & $\sqrt{ }$ & & & & & & $\sqrt{ }$ & & & \\
\hline \multirow{2}{*}{ 文 } & Slug flow monitoring & $\sqrt{ }$ & $\sqrt{ }$ & $\sqrt{ }$ & & & & & & & & \\
\hline & Hydrates formation & & $\sqrt{ }$ & & & & & & & & & \\
\hline \multirow{2}{*}{ 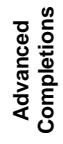 } & $\begin{array}{l}\mathrm{ICD} / \mathrm{ICV} / \mathrm{AICD} \\
\text { performance monitoring }\end{array}$ & $\sqrt{ }$ & $\sqrt{ }$ & $\sqrt{ }$ & & $\sqrt{ }$ & $\sqrt{ }$ & $\sqrt{ }$ & & $\sqrt{ }$ & & \\
\hline & ICV position & & $\sqrt{ }$ & & & & $\sqrt{ }$ & $\sqrt{ }$ & & $\sqrt{ }$ & & \\
\hline \multirow{4}{*}{ 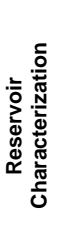 } & $\begin{array}{l}\text { Structural features } \\
\text { (faults, folds, etc) }\end{array}$ & & $\sqrt{ }$ & & & & & & $\sqrt{ }$ & & & \\
\hline & Boundaries & & $\sqrt{ }$ & & & & & & $\sqrt{ }$ & & & \\
\hline & Saturation Profiles & & & & & & & & & & & $\sqrt{ }$ \\
\hline & Well test & $\sqrt{ }$ & & & & $\sqrt{ }$ & $\sqrt{ }$ & & & & & \\
\hline
\end{tabular}

Table 3.3 - Measurement types and application areas

T- temperature, P- pressure, A- acoustic, V- vibration, S- seismic, $\varepsilon$ - strain, Q- flow and EM- electromagnetic (resistivity). 


\section{4 Numeric Non-Isothermal Dynamic Well-Reservoir Model}

Several researchers have published results showing the need of wellreservoir coupled simulation. (Vicente, Sarica et al. 2001) showed in his isothermal model that the traditional approach of decoupling wellbore flow from reservoir flow in horizontal wells do not capture the interaction between them at early times. (Grubert, Wan et al. 2009) showed that the impact of completion design in the long-term well performance is not correctly achieved without two tier coupling between well and reservoir. (Alberts, Belfroid et al. 2007) analyzed the dynamic behavior in the well and reservoir identifying the time and space scales at which the well-reservoir coupling becomes important. In Figure 4.1, a time-space map from that work is reproduced in order to show the spatial and temporal relationship among common production processes. It is possible, for example, to see that well clean-up and reservoir transients have strong coupling and then should not be analyzed separately (coincide completely). This simple idea can illustrate when one should consider well-reservoir coupling.

Multilayer reservoir well testing models have been developed and used successfully during the past two decades. The advances in downhole monitoring systems in recent years have motivated new testing and analysis techniques. Using transient temperature and pressure data (Sui, Zhu et al. 2008) developed a numerical simulator for qualitative analysis of changes in permeability and skinfactor in a multilayered vertical well. They indicated that trasient temperature can be more informative than pressure due to its sensitivity to damage radius and permeability. (Duru and Horne 2010) have found out that properties such as porosity and saturation could be estimated in an inverse problem from pressure and temperature transient analysis which are not available from conventional pressure transient analysis tools. (Valiullin, Ramazanov et al. 2009) highlighted the importance of TTA (Temperature Transient Analysis) especially when the barothermal effect (transient fluid compression/expansion at early times) takes place for well test analysis. 


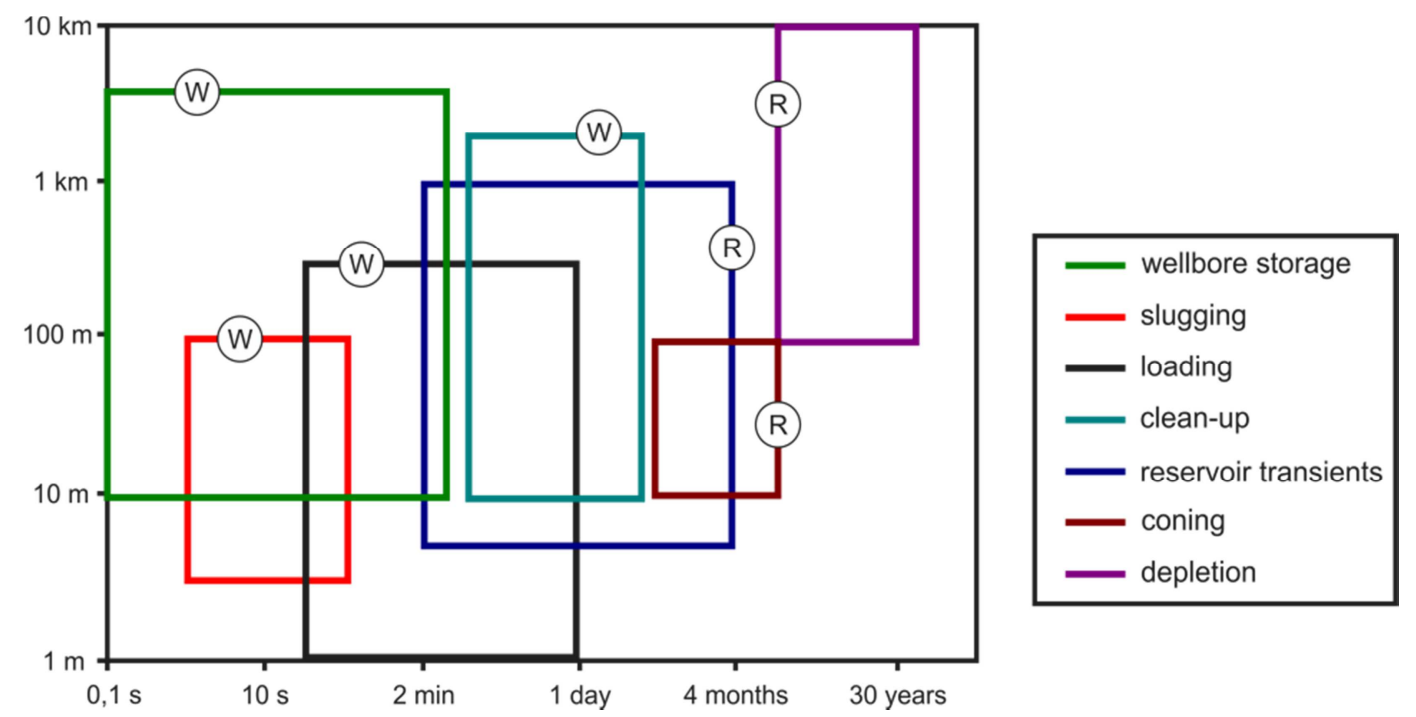

Figure 4.1 - Well/Reservoir mutual (Alberts, Belfroid et al. 2007)

TTA is not a mature technique and all possibilities have not been explored yet. A basic analysis workflow is available for sandface temperature of a single layer for a horizontal well (Muradov and Davies 2011). It assumes that downhole pressure and temperature measurements and PVT data are available. Its applicability depends on the observation of the initial temperature change period. In addition, when the formation and fluid thermal properties are known then TTA is sufficient to estimate fluid and reservoir flow properties. Otherwise, the multilayer problem is more complex due to every downstream temperature response being affected by the upstream ones. Two major issues should be overcome to solve this problem: transient heat losses and transient flow rates. Transient heat losses depend on the rate of the fluid heat loss due to the difference between the temperature of this fluid and the formation. Assuming the zonal temperatures are stable before and shortly after the transients than a HTC (Heat Transfer Coefficient) between annulus and formation can be defined and used. Transient zonal flow rates can be assumed constant during the transient period because their fluctuations have only a second order impact on the temperature signals (Muradov and Davies 2012).

Figure 4.2 shows pressure and temperature behavior of a producing horizontal well through six sensors placed in different positions. It shows clearly distinct temperature change and practically no change in pressure. In addition, temperature transients cannot be seen from all the sensors which indicate that they are affected by upstream flowing zones. 

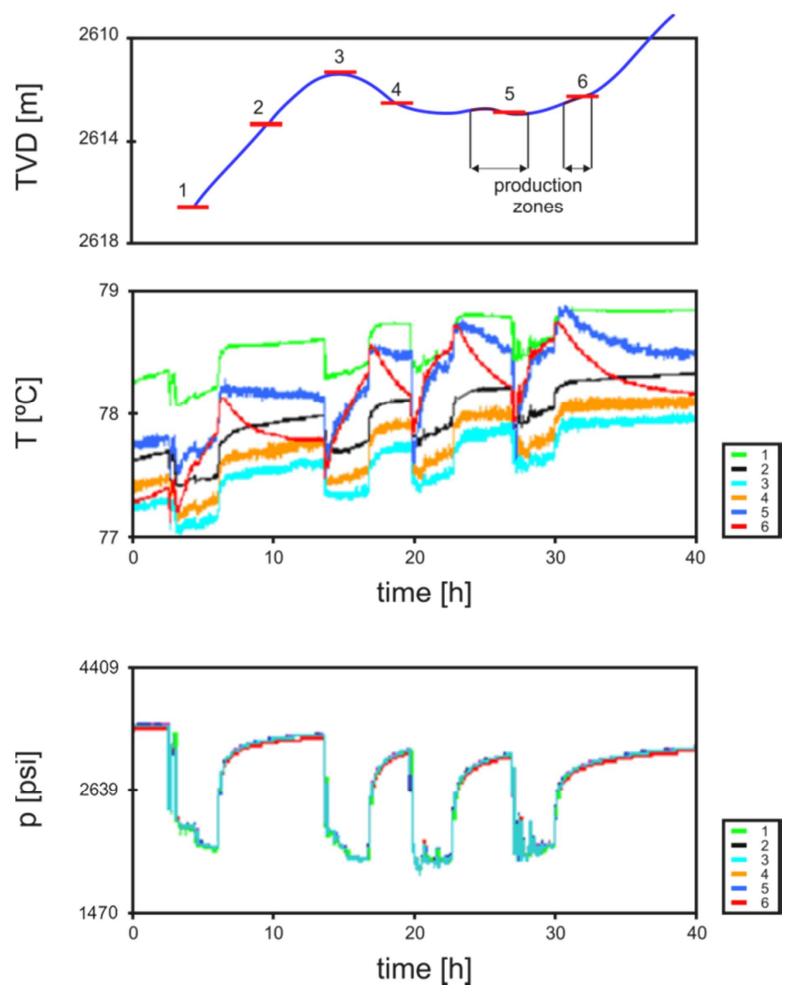

Figure 4.2 - Sensors positions and their response in a producing horizontal well (Valiullin, Ramazanov et al. 2009)

A real four zone horizontal IW pressure and temperature transient responses are shown in Figure 4.3 and Figure 4.4. The transient was created by a cycle of the wellhead choke keeping all four ICVs open. Oil, water, and gas were being produced at that time and zones 1 and 4 correspond to heel and toe respectively. The zonal pressure response for all PDGs is about the same and the differences observed happen due to friction losses. The result is in fact an average value for the well instead of the impact in each zone due to the pressure. The zonal temperature response does not have the same behavior and a relevant zone can be differentiated. It should be noted that the toe zone is the only one that is not affected by the others, representing the sandface pure response as can also be verified in Figure 4.2. A method for IW temperature distribution analysis requires that multiphase flow effects and reservoir-well thermal interaction effects to be accounted for (Muradov and Davies 2012). 


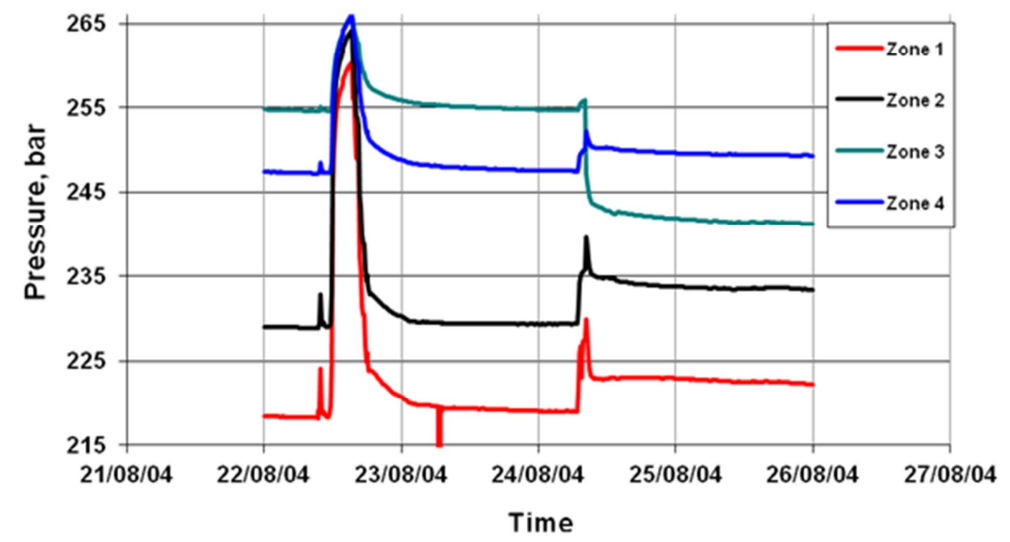

Figure 4.3 - Zonal IW transient pressure response (Muradov and Davies 2012).

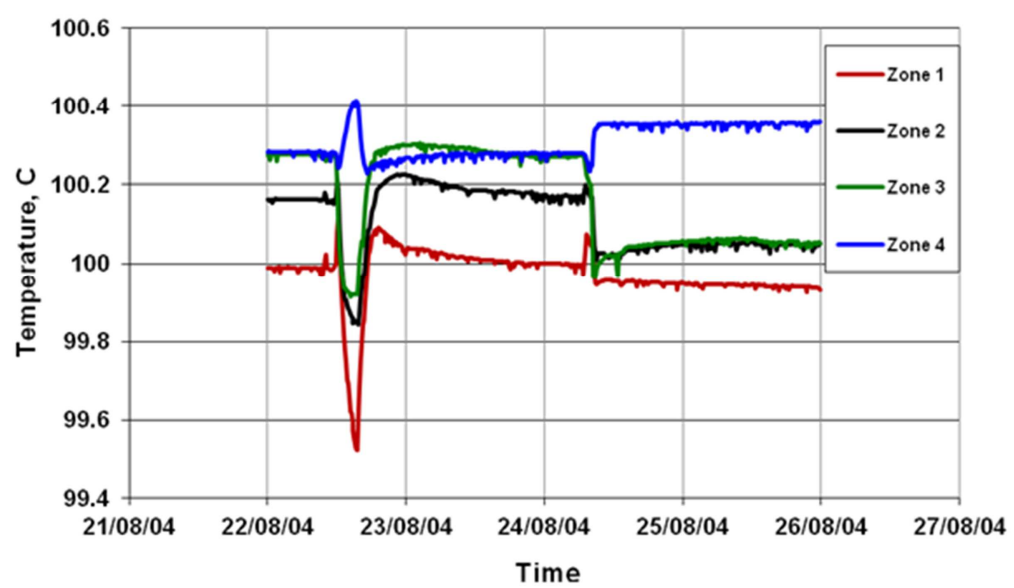

Figure 4.4 - Zonal IW transient temperature response (Muradov and Davies 2012).

The pressure and temperature responses of the toe zone as shown in Figure 4.3 and Figure 4.4 is presented in Figure 4.5 for verification purposes only. They are the sandface pure response as stated and discussed previously.

Analytical and numerical studies towards a thermal multiphase flow model in pipes and wellbore have been published in the literature for a long time. (Ramey Jr. 1962) presented the first single phase model which provides temperature as a function of time and depth. (Alves, Alhanati et al. 1992) added the two phase flow and well inclination in the model. (Hasan and Kabir 2002) have generalized the model to gas, oil and two phase flow taking into account Joule-Thomson effect and kinetic energy for the wellbore/formation system. (Yoshioka, Zhu et al. 2005) presented a multiphase steady state temperature and 
pressure model along the wellbore in horizontal wells. (Muradov and Davies 2009) proposed a general multiphase steady state well temperature model which includes simultaneous fluid flow and heat transfer not only in the annulus and tubing but also in the formation through more rigorous varying heat transfer coefficients. It is at least to date the most complete model for IWs. The model was derived from the total energy and mass balance equations assuming known pressure distribution.

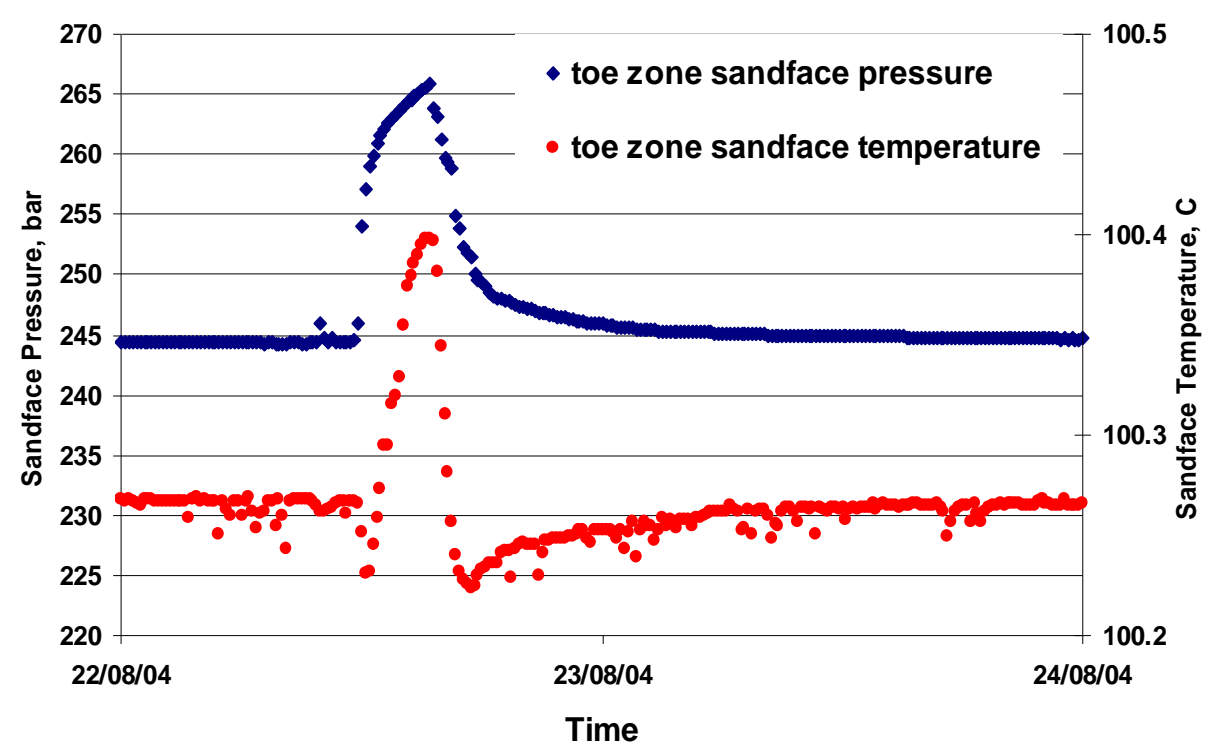

Figure 4.5 - Pressure and temperature transient sandface pure response in a horizontal IW.

We propose a numerical model for a multilayered reservoir and vertical well equipped with intelligent completion (Silva, Muradov et al. 2012). It combines the reservoir temperature model by (Sui, Zhu et al. 2008) including the necessary modifications for the two phase formulation (Appendix D), and the well temperature model by (Muradov and Davies 2008). The classic diffusivity equation by (Al-Khalifah, Aziz et al. 1987) is used for the reservoir pressure model. Pressure drop model including inflow effect by (Ouyang and Aziz 1998) is used for the well pressure model despite its small impact in vertical wells. Finally, the pressure and temperature loss due to the presence of an inflow control valve is modeled for slighted compressible fluids. The reservoir and well equations are discretized in time by implicit Euler and in space through finite element method 
over a nonuniform structured mesh. The resulting system of nonlinear algebraic equations is solved fully coupled by Newton's method.

\section{1}

\section{Well-Reservoir Model}

The non-isothermal dynamic well-reservoir model is divided in layers. Each layer is modeled as an adiabatic cylindrical-shaped reservoir experiencing transient flow and a well segment experiencing transient flow through annulus and tubing. Well segment transient is faster than reservoir thus it was considered quasi-steady-state allowing the use of heat transfer coefficients (Appendix C). The former is equipped with or without an ICV and two isolation packers with the first layer being the only exception, as shown in Figure 4.6.

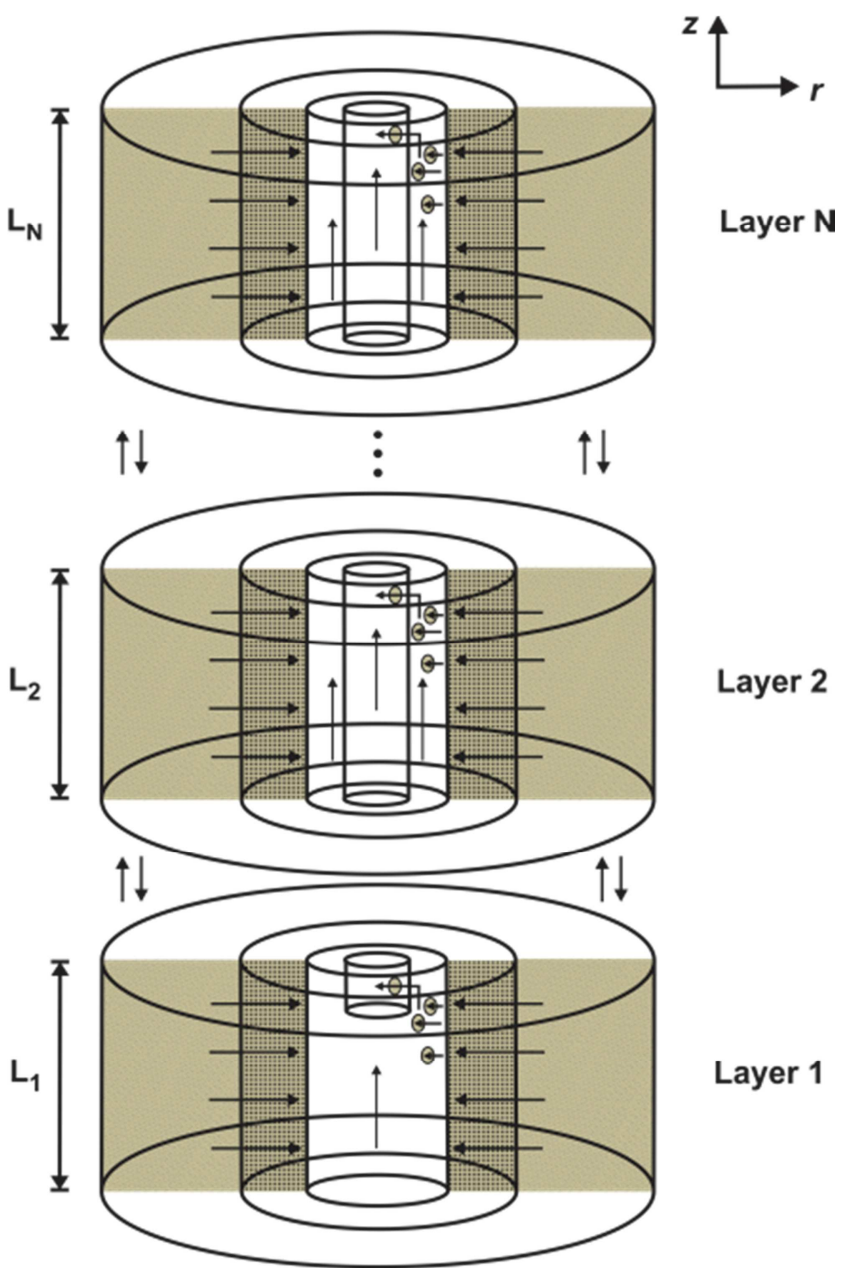

Figure 4.6 - Well-reservoir model geometry.

Mutual influences among each layer such as crossflow and thermal conduction are also taken into account. Pressure equilibrium and constant total 
flow is assumed at the well head but without storage coefficients or skin effect being considered (the model is limited to the reservoir region).

The model studied is two dimensional for the reservoir and one dimensional for the well. Reservoir is considered homogeneous, anisotropic $\left(k_{h} \neq k_{v}\right)$ and twophase (oil/water or gas/liquid) saturated with the initial pressure above the bubble point. The well is vertical and drift-flux multiphase model (Appendix B) is applied through correlations for the gas/liquid case (Ghajar and Tang 2010) where the initial rates are given. In liquid/liquid case it simplifies to homogenous multiphase model. Mass balance equations are derived using weighted properties over volume fractions for the annulus and tubing. Pressure drop model is applied with corrections due to inflow (Ouyang and Aziz 1998). Energy balance neglects kinetic energy and viscous shear energy terms (Yoshioka, Zhu et al. 2005) but heat transfer for annulus, tubing and near wellbore region are taken into account (Muradov and Davies 2008).

\subsection{1}

\section{Reservoir Layer Model (derived in appendix D)}

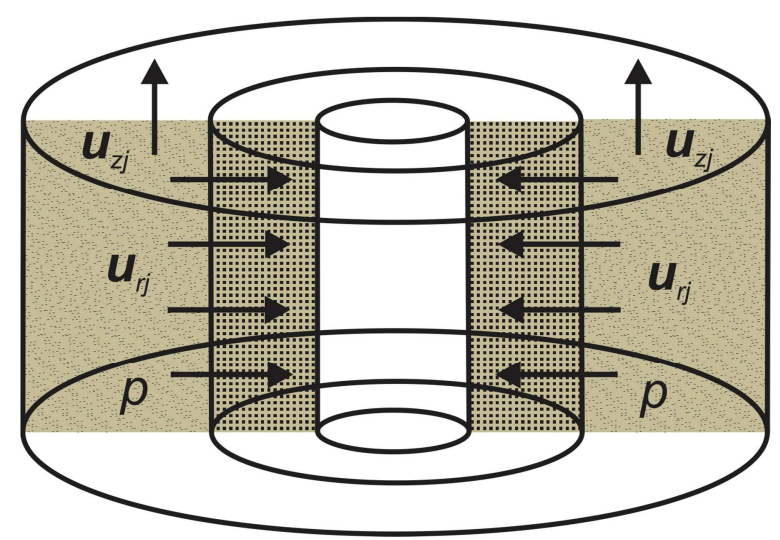

Figure 4.7 - Cylindrical-shaped reservoir layer.

Assumptions made:

- Rock and fluids are slightly compressible;

- Porous media is considered anisotropic $\left(k_{v} \neq k_{h}\right)$;

- Porous media is considered homogeneous;

- Capillarity pressure can be neglected $\left(p_{w}=p_{o}=p_{g}=p\right)$;

- Fluid saturations are assumed constant during the simulation period;

- Total compressibility times pressure is much smaller than one $\left(c_{t} p\right.$ $<<1)$; 
- Immiscible fluids;

- Well segment length is small when compared with the total length of the well;

- Total well flow rate is constant;

- Control volume is in local thermal equilibrium ;

- Rock density is constant $\left(d U_{f} \approx d H_{f}=C p_{f} d T_{f}\right)$;

- The following approximation is valid:

$$
d H=C_{p} d T+\frac{1}{\rho}(1-\beta T) d p
$$

Mass balance and Darcy's equations yields:

$$
\phi c_{t} \frac{\partial p}{\partial t}=\nabla \cdot\left(\lambda_{t} \boldsymbol{k} \cdot\left(\nabla p-\rho_{t} \mathrm{~g} \nabla z\right)\right)
$$

Where

$$
\begin{aligned}
& c_{t}=c_{f}+\sum_{j} c_{j} S_{j} \\
& \rho_{t}=\sum_{j} \rho_{j} \frac{\lambda_{j}}{\left(\sum_{j} \lambda_{j}\right)} \\
& \lambda_{t}=\sum_{j} \lambda_{j}
\end{aligned}
$$

General energy balance equation yields:

$$
\begin{aligned}
\left(\rho C_{p}\right)_{t} & \frac{\partial T}{\partial t}-\phi(\beta)_{t} T \frac{\partial p}{\partial t}-\phi c_{f}\left(p+\rho_{f} C_{p f} T\right) \frac{\partial p}{\partial t}= \\
& -\left(\rho C_{p}\right)\left(\lambda_{t} \boldsymbol{k} \cdot\left(\nabla p-\rho_{t} g \nabla z\right)\right) \cdot \nabla \mathrm{T}- \\
& -(1-(\beta) T)\left(\lambda_{t} \boldsymbol{k} \cdot\left(\nabla p-\rho_{t} g \nabla z\right)\right) \cdot \nabla p+\nabla \cdot\left(\boldsymbol{k}_{T} \boldsymbol{\nabla T}\right)
\end{aligned}
$$

Where

$$
\begin{aligned}
& \boldsymbol{k}_{T}=\phi \sum_{j} S_{j} \boldsymbol{k}_{j}+(1-\phi) \boldsymbol{k}_{f} \\
& \left(\rho C_{p}\right)_{t}=\phi \sum_{j} \rho_{j} S_{j} C_{p j}+(1-\phi) \rho_{f} C_{p f} \\
& \left(\rho C_{p}\right)=\sum_{j} \rho_{j} S_{j} C_{p j} \\
& (\beta)_{t}=\sum_{j} S_{j} \beta_{j} \\
& (\beta)=\sum_{j} \beta_{j}
\end{aligned}
$$




\subsection{2}

\section{Well Segment Model}

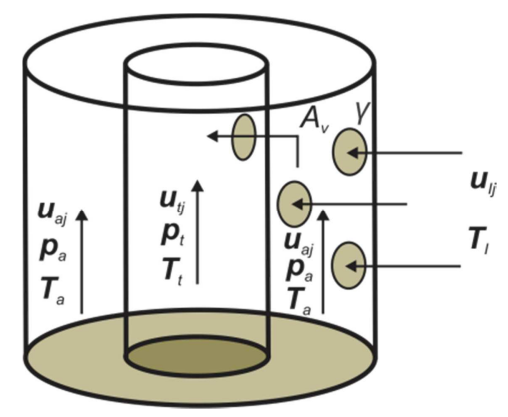

Figure 4.8 - Annulus and tubing in a well segment.

Assumptions made:

- Phase fractions $\left(y_{l}\right)$ are given for liquids and comes from (Ghajar and Tang 2010) correlations for gas/liquids $\left(x_{g}\right)$ for given rates;

- Pressure drop model with correction due to inflow is applied (Ouyang and Aziz 1998);

- The total energy in the well segment does not change;

- The well segment transient is fast when compared to the reservoir;

- There is only one phase $j$ ' dissolved in other phase $j$;

- The solubility change due to temperature is much smaller than the one due to pressure.

\subsubsection{1}

\section{Mass balance}

Annulus:

$\frac{\partial(w)_{a}}{\partial z}=\left(w^{\prime}\right)_{I}$

Where

$$
\begin{aligned}
& \left(w^{\prime}\right)_{I}=2 \pi R_{c i} \sum_{j} \gamma \rho_{I j} y_{I j} \boldsymbol{u}_{I j} \\
& (w)_{a}=\pi\left(R_{c i}^{2}-R_{t o}^{2}\right) \sum_{j} \rho_{a j} y_{a j} \boldsymbol{u}_{a j}
\end{aligned}
$$

$\gamma$ - Open radial flow area over total radial flow area.

\section{Tubing:}


$\frac{\partial(w)_{t}}{\partial z}=0$

Where

$(w)_{t}=\pi R_{t i}^{2} \sum_{j} \rho_{t j} y_{t j} u_{t j}$

\subsubsection{2}

\section{Momentum balance}

Annulus:

$$
\frac{\partial p_{a}}{\partial z}=\frac{\partial\left(\rho_{m a} u_{m a}^{2}\right)}{\partial z}+\frac{2 f_{F a} \boldsymbol{u}_{m a}^{2} \rho_{m a}}{D_{h a}}+\rho_{m a} g \sin \theta
$$

Tubing:

$$
\frac{\partial p_{t}}{\partial z}=\frac{\partial\left(\rho_{m t} u_{m t}^{2}\right)}{\partial z}+\frac{2 f_{F t} \boldsymbol{u}_{m t}^{2} \rho_{m t}}{D_{t}}+\rho_{m t} g \sin \theta
$$

Where the average properties (for the annulus and tubing) are:

$$
\begin{aligned}
& \rho_{m}=\sum_{j} \rho_{j} y_{j} \\
& u_{m}=\frac{\sum_{j} \rho_{j} y_{j} u_{j}}{\sum_{j} \rho_{j} y_{j}}
\end{aligned}
$$

The frictional force per unit of volume between a single phase fluid and the wall is evaluated through the friction factor $\left(f_{F t}\right.$ and $\left.f_{F a}\right)$ which is calculated by using classic friction factor correlations for pipe flow (Appendix A). Chen correlation is used for this purposes as recommended in (Hasan and Kabir 2002). It is not a strong effect in vertical wells but should be considered for high rate wells.

For a gas-liquid flow in a vertical pipe the superficial gas velocity (cross sectional area averaged velocity) is expressed as a sum of the distribution coefficient $\left(C_{o}\right)$ and the gas drift velocity $\left(u_{g m}\right)$. It captures not only the mechanisms of higher concentration of gas near the center of the pipe $\left(C_{o} u_{m}\right)$ but also the tendency of buoyancy $\left(u_{g m}\right)$. This explains why gas inside a vertical pipe moves faster than liquid.

$$
u_{g}=C_{o} u_{m}+u_{g m}
$$


The average mixture velocity is defined as the sum of the superficial velocities:

$$
u_{m}=u_{s g}+u_{s l}=\left(1-y_{l}\right) u_{g}+y_{l} u_{l}
$$

Drift-flux model (Appendix B) is used through correlations from (Ghajar and Tang 2010) to obtain $C_{o}, u_{g m}$ and $y_{l}$ from given rates.

\subsubsection{3}

\section{Temperature model}

The temperature model (Muradov and Davies 2008) is derived from total energy balance and mass balance equations. It was simplified here to fit the assumptions made. Continuous inflow from the formation, heat transfer tubingannulus and annulus-formation, and one phase change is accounted for in the model presented in (4.7) and (4.8). The third RHS term indicated by index $I$ in (4.7) represents the radial mass inflow per unit of length ( $\left.w^{\prime}\right)$. The last RHS terms in (4.7) and (4.8) accounts for phase change from $j$ ' to $j$ and Joule-Thomson effect where $H_{\text {lat,jj' }}$ is the latent heat to change from the phase $\mathrm{j}$ ' to $\mathrm{j}$ and $k_{J T}$ is the JouleThomson coefficient which is: $k_{J T}=\frac{1}{\rho C_{p}}(\beta T-1)$ for liquid and $k_{J T}=\frac{T}{\rho C_{p} z}\left(\frac{\partial z}{\partial T}\right)_{p}$ for gas. It should be noted that there is only one phase dissolved in other and $R_{s}$ is the gas-oil solubility ratio from black oil model. All coefficients in brackets are average coefficients for annulus and tubing. Heat transfer mechanisms among tubing, annulus and formation are accounted for using heat transfer coefficients $\left(U_{t a}\right.$ and $\left.U_{a f}\right)$.

Annulus:

$$
\begin{aligned}
& \left(w C_{p}\right)_{a} \frac{\partial T_{a}}{\partial z}=2 \pi R_{c i}(1-\gamma) U_{a f}\left(T_{f}-T_{a}\right)-2 \pi R_{t i} U_{t a}\left(T_{a}-T_{t}\right)+\left(w^{\prime} C_{p}\left(T_{I}-T_{a}\right)\right)_{I}+ \\
& +(w)_{a} g \sin \theta+\left[\left(w C_{p} k_{J T}\right)_{a}+(w)_{a, j^{\prime}} H_{l a t, j j^{\prime}}\left(\left.\frac{\rho_{a j}}{\rho_{a j^{\prime}}} \frac{\partial R_{s}}{\partial p_{a}}\right|_{T_{a}}\right)\right] \frac{\partial p_{a}}{\partial z}
\end{aligned}
$$

Tubing: 


$$
\begin{aligned}
& \left(w C_{p}\right)_{t} \frac{\partial T_{t}}{\partial z}=2 \pi R_{t i} U_{t a}\left(T_{a}-T_{t}\right)+(w)_{t} g \sin \theta+ \\
& +\left[\left(w C_{p} k_{J T}\right)_{t}+(w)_{t, j^{\prime}} H_{l a t, j j^{\prime}}\left(\left.\frac{\rho_{t j}}{\rho_{t j^{\prime}}} \frac{\partial R_{s}}{\partial p_{t}}\right|_{T_{t}}\right)\right] \frac{\partial p_{t}}{\partial z}
\end{aligned}
$$

\subsection{3}

\section{Initial and Boundary Conditions}

Two sets of initial and boundary conditions are defined. One set is used for the first layer and another for any intermediary layer. The first layer is assumed to be the deepest one experiencing uniform flow without tubing but with or without a valve. The intermediary one represents a conventional IW segment. The presence of a valve is taken into account as a different boundary condition for the next layer (Table 4.1 and 4.2). Crossflow and heat transfer between layers are also considered exceptions for the first and last layers (Figure 4.6). Transients can be created by the wellhead choke or by the ICVs. Total flow is kept constant (the sum of all layer flows) if the transient is created by the ICVs. Equation 4.14 or 4.15 are used to account for the flowrate change. In this case there will be flowrate

\begin{tabular}{|c|c|c|}
\hline \begin{tabular}{|l|} 
Layer 1 \\
\end{tabular} & Initial Conditions & Boundary Conditions \\
\hline \multirow[b]{2}{*}{ Reservoir } & $\left.T_{1}^{(t=0)}\right|_{(r, z)}=T_{e_{1}}-g_{T} z$ & $\begin{array}{l}\left.T_{1}^{(t)}\right|_{\left(r=R_{e} z\right)}=T_{e_{1}}-g_{T} z \\
-k_{T_{1}} \frac{\partial T_{1}^{(t)}}{\partial r} l_{\left.r=R_{c^{2}}\right)}=U_{w b f}^{(t)}\left(T_{1}^{(t)}-T_{w b}^{(t)}\right) \\
\left.k_{T_{2}} \frac{\partial T_{2}^{(t)}}{\partial z}\right|_{(r, z=0)}=k_{f_{1}} \frac{\partial T_{1}^{(t)}}{\partial z} l_{r z=L)} \\
\left.T_{1}^{(t)}\right|_{r, z=0)}=T_{e_{1}}\end{array}$ \\
\hline & $\left.p_{1}^{(t=0)}\right|_{(r, z)}=p_{e_{1}}-\rho_{t_{1}} g z$ & $\begin{array}{l}\left.p_{1}^{(t)}\right|_{\left(r=R_{e}, z\right)}=p_{q}-\rho_{t_{1}} g z \\
-\left.\frac{k_{h}}{\mu} \frac{\partial p_{1}^{(t)}}{\partial r}\right|_{\left(r=R_{i, 2}\right)}=\frac{q_{1}}{2 \pi R_{i c} L_{1}} \\
\left.\left(\frac{k_{v}}{\mu}\right)_{2} \frac{\partial p_{2}^{(t)}}{\partial z}\right|_{(r, z=0)}=\left.\left(\frac{k_{v}}{\mu}\right)_{1} \frac{\partial p_{1}^{(t)}}{\partial z}\right|_{\left(r, z=L_{1}\right)} \\
\left.p_{1}^{(t)}\right|_{(r, z=0)}=p_{e_{1}}\end{array}$ \\
\hline \multirow{3}{*}{ Well } & $T_{w b}^{(1=0)} k_{z 2}=T_{e_{1}}-g_{T} z$ & $\left.T_{w b}^{(t)}\right|_{(z=0)}=T_{e_{1}}$ \\
\hline & $\left.p_{w b}^{(t=0)}\right|_{(z)}=p_{e_{1}}-\rho_{m} g z$ & $p_{w b}{ }^{(t)} l_{z=0)}=p_{e_{1}}$ \\
\hline & $\left.u_{w b}^{(t=0)}\right|_{(z)}=0$ & $\left.u_{w b}{ }^{(t)}\right|_{(z=0)}=0$ \\
\hline
\end{tabular}
changes in the other zones to keep the total flowrate constant.

Table 4.1 - IC and BC for layer 1 


\begin{tabular}{|c|c|c|c|c|}
\hline Layer i & \multicolumn{2}{|c|}{ Initial Con ditions } & \multicolumn{2}{|r|}{ Boundary Conditions } \\
\hline \multirow{2}{*}{ Reservoir } & \multicolumn{2}{|l|}{$\left.T_{j}^{(t=0)}\right|_{r, z)}=T_{e_{j}}-g_{T} z$} & \multicolumn{2}{|c|}{$\begin{array}{l}\left.T_{j}^{(t)}\right|_{\left.r=R_{e} z\right)}=T_{e_{j}}-g_{T} z \\
-\left.k_{T_{j}} \frac{\partial T_{j}^{(t)}}{\partial r}\right|_{\left(r=R_{\left.c_{i}, z\right)}\right.}=U_{w b f_{j}}^{(t)}\left(T_{j}^{(t)}-T_{a_{j}}^{(t)}\right) \\
\left.k_{T_{j / j}} \frac{\partial T_{j+1}^{(t)}}{\partial z}\right|_{r, z=0)}=\left.k_{T_{j}} \frac{\partial T_{j}^{(t)}}{\partial z}\right|_{\left.r, z=L_{j}\right)} \\
\left.T_{N}^{(t)}\right|_{\left(r, z=L_{N}\right)}=T_{e_{N}}-g_{T} L_{N} \\
\end{array}$} \\
\hline & \multicolumn{2}{|l|}{$\left.p_{j}^{(t=0)}\right|_{(r, z)}=p_{e_{j}}-\rho_{t_{j}} g z$} & $\begin{array}{l}\left.p_{j}^{(t)}\right|_{\left(r=R_{e}, z\right)}=p_{e_{j}}-\rho_{t_{j}} g z \\
-\left.\frac{k_{h}}{\mu} \frac{\partial p_{j}^{(t)}}{\partial r}\right|_{\left(r=R_{c i z}\right)}=\frac{q_{j}}{2 \pi R_{c} L_{j}} \\
\left.\left(\frac{k_{v}}{\mu}\right)_{j+1} \frac{\partial p_{j+1}^{(t)}}{\partial z}\right|_{(r, z=0)}=\left(\frac{k_{v}}{\mu}\right) \\
\left.p_{N}^{(t)}\right|_{\left.r, z=L_{N}\right)}=p_{e_{N}}-\rho_{t_{N}} g L_{N}\end{array}$ & $\left.\int_{j} \frac{\partial p_{j}^{(t)}}{\partial z}\right|_{\left(r, z=L_{j}\right)}$ \\
\hline \multirow{3}{*}{ Well } & $\left.T_{a_{j}}^{(t=0)}\right|_{(z)}=T_{e_{j}}-g_{T} z$ & $\left.T_{t_{j}}^{(t=0)}\right|_{(z)}=T_{e_{j}}-g_{T} z$ & $\left.T_{a_{j}}^{(t)}\right|_{\left.z_{j}=0\right)}=\left.T_{j}^{(t)}\right|_{\left.r=R_{c i c}, z_{j}=0\right)}$ & 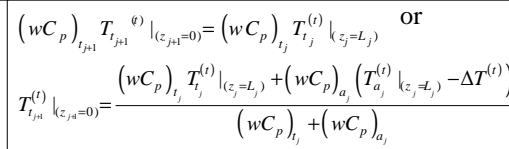 \\
\hline & $\left.p_{a_{j}}^{(l=0)}\right|_{(z)}=p_{e_{j}}-\rho_{m_{j}} g z$ & $\left.p_{t_{j}}^{(t=0)}\right|_{(z)}=p_{e_{j}}-\rho_{m_{j}} g z$ & $\left.p_{a_{j}}^{(t)}\right|_{\left(z_{j}=0\right)}=\left.p_{j}^{(t)}\right|_{\left(r=R_{i j}, z_{j}=0\right)}$ & 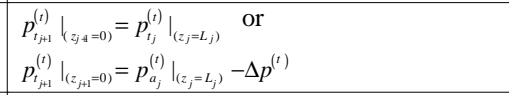 \\
\hline & $\left.u_{a_{j}}{ }^{(t=0)}\right|_{(z)}=0$ & $\left.u_{t_{j}}^{(t=0)}\right|_{z z}=0$ & $\left.u_{a_{j}}^{(t)}\right|_{\left(z_{j}=0\right)}=0$ & 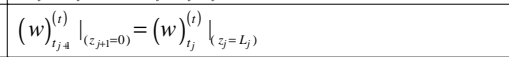 \\
\hline
\end{tabular}

Table 4.2 - IC and BC for layer $j$.

\section{2}

\section{Heat transfer coefficients}

A well-reservoir segment is shown in Figure 4.9 with its radial layers: tubing, annulus, cement and formation. The heat transfer mechanisms among them can be described by conduction, convection and radiation. The overall heat transfer coefficients for the radial geometry are based on the ones proposed by Muradov (Muradov and Davies 2008). They are divided in tubing-annulus and annulus formation heat transfer coefficients (Appendix C).

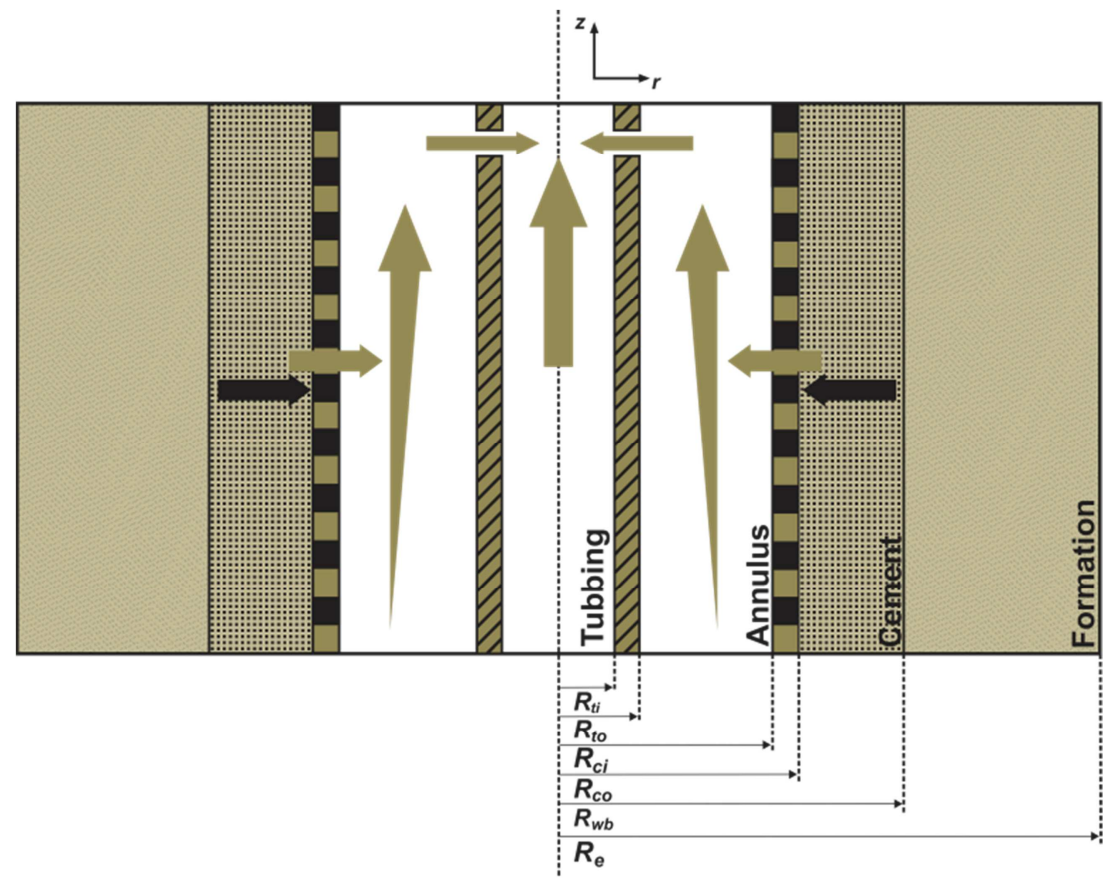

Figure 4.9 - Well-reservoir segment and its radial layers 


\subsection{1 Heat transfer coefficient between annulus and tubing}

Convection, conduction and radiation due to the fluids in the annulus (the latter was neglected here) and tubing as well as the convection and conduction through the tubing wall are taken into account:

$$
U_{t a}=\frac{1}{\frac{1}{h_{t}}+\frac{R_{t i}}{K_{t u b i n g}} \ln \left(\frac{R_{t o}}{R_{t i}}\right)+\frac{R_{t i}}{R_{t o} h_{a}}}
$$

The convection coefficients $h_{t}$ and $h_{a}$ are based on correlations (Sieder and Tate 1936; Churchill and Chu 1975; Churchill and Chu 1975). Tubing and annulus can be experiencing stagnant, laminar, and transitional or turbulent flow. The appropriate coefficient is chosen based on Reynolds number and Grashof number. We use the fact that superposition is valid for laminar flow with constant heat flux and simultaneously developing velocity (Heaton, Reynolds et al. 1964).

\subsection{2}

\section{Heat transfer coefficient between annulus to formation}

Convection in the annulus (same as above), conduction through the casing wall and cement as well as conduction to the formation is calculated by:

$$
U_{a f}=\frac{1}{\frac{1}{h_{a}}+\frac{R_{c i}}{K_{c a s i n g}} \ln \left(\frac{R_{c o}}{R_{c i}}\right)+\frac{R_{c i}}{K_{c e m e n t}} \ln \left(\frac{R_{w b}}{R_{c o}}\right)}
$$

\section{3}

\section{Inflow control valve}

An inflow control valve is a downhole valve which the basic mechanical project is similar to a sliding sleeve valve but with remote control. It reminds a cage style trim control valve which provides a balanced plug and a sliding ringtype seal. There are two kinds of flow area profiles available: oblong (continuous flow area) or circular (discrete flow area) as shown in Figure 4.10. The flow area profiles can be exposed to the flow completely (closed-open) or partially (multiposition or continuous). In the multi-position case, there are typically 2 to 6 intermediary positions with different areas. Both uses equal percentage as flow control strategy. Due to an industry standard the max valve area (full open) is equal to an equivalent tubing area, e. g. a 3 1/2" valve has its full open area equals 
to a $3 \frac{1 / 2}{}$ " tubing area (for same tubing weight). It should be noted that the valve flow area profile always depends on the application, in other words, on the fluids involved, pressure, temperature and expected flow rate. A simplified approach for ICV flow area design based on nodal analysis and IPR (Inflow Performance Relationship) can found in (Konopczynski and Ajayi 2004).
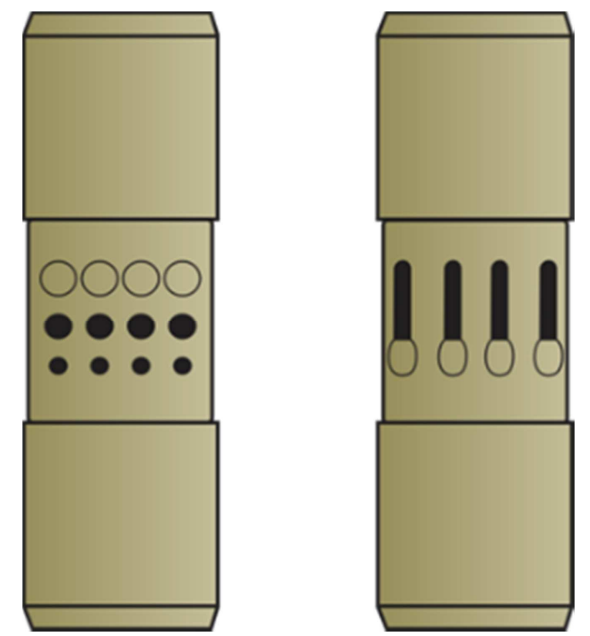

Figure 4.10 - ICV.

The flow control valve model used in this development is achieved using Bernoulli and mass balance equations for an ideal incompressible fluid using the geometry shown in Figure 4.11:

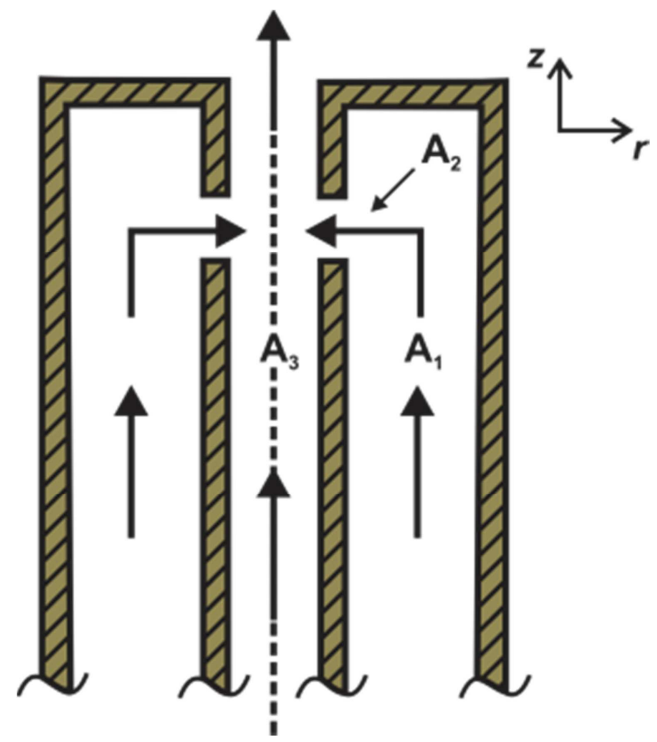

Figure 4.11 - Geometry used in the valve model $(\mathrm{A} 2=\mathrm{A} 3)$. 


$$
(w)_{\text {ideal }}=\frac{A_{2}}{\sqrt{1-\left(\frac{A_{2}}{A_{1}}\right)^{2}}} \sqrt{2 \Delta p \rho}
$$

We obtain the approximation equation for the real incompressible fluid flow correcting for viscous losses and vena contracta through the valve discharge coefficient $\left(C_{d}\right)$ :

$$
C_{d} \equiv \frac{(w)_{\text {real }}}{(w)_{\text {ideal }}}
$$

Finally to compensate compression $(Y)$ and thermal $\left(F_{a}\right)$ expansion effects additional coefficients can be added:

$$
(w)_{\text {real }}=\frac{C_{d} Y F_{a}}{\sqrt{1-\left(\frac{A_{2}}{A_{1}}\right)^{2}}} A_{2} \sqrt{2 \Delta p \rho}
$$

When the geometric factor is included $C_{d}$ (4.13) becomes flow coefficient $(C)$ :

$$
(w)_{\text {real }}=C Y F_{a} A_{2} \sqrt{2 \Delta p \rho}
$$

All factors are obtained experimentally and it is assumed $R e>10000$ and defined at each valve trim position.

For flow mixtures of gas/liquid we have chosen (Zhang, Yue et al. 2005) model assuming $\rho_{\text {liquid }} \gg \rho_{\text {gas }}$ and gas quality small:

$$
(w)_{\text {real }}=C A_{2} Y F_{a} \sqrt{\frac{2 \Delta p \rho_{l}}{0.5\left(\frac{1-y}{y}\right)^{0.95}\left(\frac{\rho_{l}}{\rho_{g}}\right)^{0.02}+1}}
$$

Both equations (4.14 and 4.15) are presented in (Oliveira, Passos et al. 2009) and they are based on ISO-5167 (Measurement of fluid flow by means of pressure differential devices inserted in circular cross-section conduits running full).

Hydraulic connection between the annulus and tubing is made by an ICV. The flow area of the valve is the same for the tubing when it is in a fully opened position as a standard industry design. The pressure in the tubing at the valve position can be derived from (4.14) neglecting compression and thermal expansion effects: 


$$
\begin{aligned}
& p_{t, d}-p_{a, u}=-\frac{\rho_{a} A_{1}^{2} u_{a}^{2}}{2 C_{d}^{2} A_{2}^{2}} \\
& \Delta p=-\frac{\rho_{a} A_{1}^{2} u_{a}^{2}}{2 C_{d}^{2} A_{2}^{2}}
\end{aligned}
$$

The annulus density and velocity used in this equation can be modeled as single phase or by averaging over volume fraction for two-phase (liquid/liquid). Two phase (gas/liquid) can be derived from (4.15) using phase fraction from (Ghajar and Tang 2010) but not neglecting compression and thermal expansion effects.

The temperature change in the ICV can be modeled through the isenthalpic model:

$$
\begin{aligned}
& d H=\left(\frac{\partial H}{\partial T}\right)_{p} d T+\left(\frac{\partial H}{\partial p}\right)_{T} d p \approx C_{p} d T+\frac{(1-\beta T)}{\rho} d p=0 \\
& d T=\frac{(\beta T-1)}{\rho C_{p}} d p=K_{J T} d p
\end{aligned}
$$

From (4.18) the average two-phase temperature change in the valve can be calculated as:

$$
\begin{aligned}
& \left(T_{t, d}-T_{a, u}\right)=\frac{\left(w C_{p} K_{J T}\right)}{\left(w C_{p}\right)}\left(p_{t, d}-p_{a, u}\right)=\overline{K_{J T}}\left(p_{t, d}-p_{a, u}\right) \\
& \Delta T=\overline{K_{J T}} \Delta \mathrm{p}
\end{aligned}
$$

Therefore, the fluid mixture temperature downstream the valve is:

$$
T_{t, d}=\frac{\left(w C_{p}\right)_{t, u} T_{t, u}+\left(w C_{p}\right)_{a, u}\left(T_{a, u}-\Delta T\right)}{\left(w C_{p}\right)_{t, u}+\left(w C_{p}\right)_{a, u}}
$$

The equation (4.19) is used as boundary condition for layer $j$ when layer $j-1$ is equipped with an ICV. It is assumed that there is no phase change within the valve trim and only liquids or slighted compressible fluids are flowing through at below critical velocities.

\section{4}

\section{Numerical Solution}

Variational equations are derived from the differential equations of the wellreservoir coupled model and then discretized in time by implicit Euler and in 
space through finite element method on a nonuniform structured mesh. The resulting system of nonlinear algebraic equations is solved simultaneously by Newton's method using a preconditioned GMRES iterative algorithm as solver.

\subsection{1}

\section{Variational Formulation}

The well-reservoir equations are solved for $p$ and $T$ in the reservoir and for $p_{a}, p_{t}, u_{a}, u_{t}$ and $T_{a}, T_{t}$ (annulus and tubing of the well). In order to assure the mathematical consistence of the formulation, we need to state the regularity properties of these variables. Let us say that the pressure and temperature fields of the reservoir and the velocity, pressure and temperature ones of the well are solution to the variational problem in the domain $\Omega=\Omega_{\mathrm{r}} \times \Omega_{\mathrm{w}}$ which we define belonging to the following solution subspace:

$\mathbf{S}:=\underset{P r}{\operatorname{Pr}} \mathbf{T}_{r} \times \mathbf{V}_{w} \times \mathbf{P}_{w} \times \mathbf{T}_{w}:=$ $\left\{\left(p, T, \boldsymbol{u}_{a}, \boldsymbol{u}_{t}, p_{a}, p_{t}, T_{a}, T_{t}\right) \mid p \in \mathbf{P}_{r} ; T \in \mathbf{T}_{r} ; \boldsymbol{u}_{a}, \boldsymbol{u}_{t} \in \mathbf{V}_{w} ; p_{a}, p_{t} \in \mathbf{P}_{w} ; T_{a}, T_{t} \in \mathbf{T}_{w}\right\}$

Where:

$\mathbf{P r}:=\left\{p \in \mathbf{H}^{1}\left(\Omega_{r}\right)|p|_{\partial \Omega r}=p_{\partial \Omega r}\right\}$

$\mathbf{T r}:=\left\{T \in \mathbf{H}^{0}\left(\Omega_{r}\right)\right\}$

$\mathbf{V} \mathbf{r}:=\left\{\boldsymbol{u}_{w} \in \mathbf{H}^{1}\left(\Omega_{w}\right)\left|\boldsymbol{u}_{w}\right|_{\partial \Omega w}=\boldsymbol{u}_{\partial \Omega w}\right\}$

$\mathbf{P} r:=\left\{p_{w} \in \mathbf{H}^{0}\left(\Omega_{w}\right)\right\}$

$\mathbf{T} \mathbf{r}:=\left\{T_{w} \in \mathbf{H}^{0}\left(\Omega_{w}\right)\right\}$

Where $\mathbf{H}^{k}(\Omega)$ is the Sobolev space of order $k$ which is defined as:

$$
\mathbf{H}^{k}(\Omega)=\left\{f \mid f \in L_{2}(\Omega), \frac{\partial f}{\partial x} \in L_{2}(\Omega) ; \ldots ; \frac{\partial^{k} f}{\partial x} \in L_{2}(\Omega)\right\}
$$

This definition states that if a function $f$ belongs to $\mathbf{H}^{k}(\Omega)$ then $\mathrm{f}$ and its derivatives up to order $\mathrm{k}$ have a finite $L_{2}(\Omega)$ norm:

$$
L_{2}(\Omega)=\left\{f: \int_{\Omega}|f|^{2} d \Omega<+\infty\right\}
$$

It should be noted that in order to ensure the uniqueness of the solution we need to prescribe Dirichlet boundary conditions on $\partial \Omega$. 
The variational formulation is equivalent to the differential formulation of the coupled well-reservoir problem where $\hat{p}$ and $\hat{T}$ are arbitrarily chosen functions for the reservoir and $\hat{\boldsymbol{u}}_{a}, \hat{p}_{a}, \hat{T}_{a}, \hat{\boldsymbol{u}}_{t}, \hat{p}_{t}$ and $\hat{T}_{t}$ are arbitrarily chosen functions for the annulus and tubing of the well. Hence, the well-reservoir problem can be stated as: find $p \in \operatorname{Pr}, T \in \mathbf{T}_{r},\left(\boldsymbol{u}_{a}, \boldsymbol{u}_{t}\right) \in \mathbf{V}_{w},\left(p_{a}, p_{t}\right) \in \mathbf{P}_{w}$ and $\left(T_{a}, T_{t}\right) \in \mathbf{T}_{w}$ such that $\hat{p} \in \mathbf{P r}, \hat{T} \in \mathbf{T w},\left(\hat{\boldsymbol{u}}_{a}, \hat{\boldsymbol{u}}_{t}\right) \in \mathbf{V}_{w},\left(\hat{p}_{\mathrm{a}}, \hat{p}_{\mathrm{t}}\right) \in \mathbf{P}_{w}$, and $\quad\left(\hat{T}_{a}, \hat{T}_{t}\right) \in \mathbf{T}_{w}$ are subjected to:

In reservoir:

$$
\begin{aligned}
& \phi c_{t} \int_{\Omega_{r}} \hat{p} \frac{\partial p}{\partial t} d v-\int_{\Omega_{r}} \nabla \hat{p} \cdot\left(\lambda_{t} \boldsymbol{k} \cdot\left(\nabla p-\rho_{t} g \nabla z\right)\right) d v+ \\
& \quad+\int_{\partial \Omega_{r}} \hat{p}\left(\lambda_{t} \boldsymbol{k} \cdot\left(\nabla p-\rho_{t} g \nabla z\right)\right) \cdot \boldsymbol{n} d a=0 \\
& \left(\rho C_{p}\right)_{t} \int_{\Omega_{r}} \hat{T} \frac{\partial T}{\partial t} d v-\phi(\beta)_{t} \int_{\Omega_{r}} \hat{T} T \frac{\partial p}{\partial t} d v-\phi c_{f} \int_{\Omega_{r}} \hat{T}\left(p+p_{f} C_{p f} T\right) \frac{\partial p}{\partial t} d v+ \\
& \quad+\left(\rho C_{p}\right) \int_{\Omega_{r}} \hat{T} \nabla T \cdot\left(\lambda_{t} \boldsymbol{k} \cdot\left(\nabla p-\rho_{t} g \nabla z\right)\right) d v+ \\
& \quad+\int_{\Omega_{r}}(1-(\beta)) T \nabla p \cdot\left(\lambda_{t} \boldsymbol{k} \cdot\left(\nabla p-\rho_{t} g \nabla z\right)\right) \hat{T} d v+\int_{\Omega_{r}} \nabla \hat{T} \cdot\left(\boldsymbol{k}_{T} \cdot \nabla T\right) d v- \\
& \quad-\int_{\partial \Omega_{r}} \hat{T}\left(\boldsymbol{k}_{T} \cdot \nabla T\right) \cdot \boldsymbol{n} d a=0
\end{aligned}
$$

In well annulus:

$$
\begin{aligned}
& A_{a} \int_{\Omega_{w}} \hat{\boldsymbol{u}}_{a} \frac{\partial(\rho y \boldsymbol{u})_{a}}{\partial z} d z-2 \pi R_{c i} \int_{\Omega_{w}} \hat{\boldsymbol{u}}_{a}(\gamma \rho y \boldsymbol{u})_{I} d z=0 \\
& \int_{\Omega_{w}} \hat{p}_{a} \frac{\partial p_{a}}{\partial z} d z-\int_{\Omega_{w}} \hat{p}_{a}\left(\frac{\partial \rho_{a} u_{a}^{2}}{\partial z}+\frac{2 f_{F a} \boldsymbol{u}_{a}^{2} \rho_{a}}{D_{h a}}+\rho_{a} g \sin \theta\right) d z=0 \\
& A_{a} \int_{\Omega_{w}}\left(\rho y \boldsymbol{u} C_{p}\right)_{a} \hat{T}_{a} \frac{\partial T_{a}}{\partial z} d z-\int_{\Omega_{w}} \hat{T}_{a}\left(2 \pi R_{c i}(1-\gamma) U_{a f}\left(T_{f}-T_{a}\right)-2 \pi R_{t i} U_{t a}\left(T_{a}-T_{t}\right)\right) d z- \\
& \quad-\int_{\Omega_{w}} \hat{T}_{a}\left(2 \pi R_{c i}\left(\gamma \rho y \boldsymbol{u} C_{p}\left(T_{I}-T_{a}\right)\right)_{I}-A_{a}(\rho y \boldsymbol{u})_{a} g \sin \theta\right) d z- \\
& \quad-A_{a} \int_{\Omega_{w}} \hat{T}_{a}\left[\left(\rho y \boldsymbol{u} C_{p} k_{J T}\right)_{a}+\left(\rho y \boldsymbol{u} C_{p}\right)_{a} H_{l a t, g o}\left(\left.\frac{\rho_{a g}}{\rho_{a o}} \frac{\partial R_{s}}{\partial p_{a}}\right|_{T_{a}}\right)\right] \frac{\partial p_{a}}{\partial z} d z=0
\end{aligned}
$$

In well tubing: 


$$
\begin{aligned}
& A_{t} \int_{\Omega_{w}} \hat{u}_{t} \frac{\partial(\rho y \boldsymbol{u})_{t}}{\partial z} d z=0 \\
& \int_{\Omega_{w}} \hat{p}_{t} \frac{\partial p_{t}}{\partial z} d z-\int_{\Omega_{w}} \hat{p}_{t}\left(\frac{\partial \rho_{t} u_{t}^{2}}{\partial z}+\frac{2 f_{F a} \boldsymbol{u}_{t}^{2} \rho_{t}}{D_{t}}+\rho_{t} g \sin \theta\right) d z=0 \\
& A_{t} \int_{\Omega_{w}}\left(\rho y \boldsymbol{u} C_{p}\right)_{t} \hat{T}_{t} \frac{\partial T_{t}}{\partial z} d z-\int_{\Omega_{w}}\left(2 \pi R_{t i} U_{t a}\left(T_{a}-T_{t}\right)+A_{t}(\rho y \boldsymbol{u})_{t} g \sin \theta\right) d z- \\
& \quad-A_{t} \int_{\Omega_{w}} \hat{T}_{t}\left[\left(\rho y \boldsymbol{u} C_{p} k_{J T}\right)_{t}+\left(\rho y \boldsymbol{u} C_{p}\right)_{t} H_{l a t, g o}\left(\left.\frac{\rho_{t g}}{\rho_{t o}} \frac{\partial R_{s}}{\partial p_{t}}\right|_{T_{t}}\right)\right] \frac{\partial p_{t}}{\partial z} d z=0
\end{aligned}
$$

Limited to the domain $\Omega$.

\subsection{2}

\section{Space and time discretization}

The space discretization process in the finite element method is essentially the choice of how to divide the domain into elements and basis functions used to describe the unknowns fields in each element. This choice should take into account influences of the variational formulation, regularity properties of the exact solution, accuracy requirements, computational effort, etc. The original infinite solution subspace is replaced by the discretized one and therefore it becomes a finite dimensional problem. Solution subspace of the new finite dimensional problem are variables or degrees of freedom in each element described by a set of points which are uniquely determined by a piecewise polynomial subspace spanned by a Lagrange basis. In addition, mixed formulation is used and therefore Babuska-Brezzi condition must be verified to ensure the existence and uniqueness of this approximated solution (Brezzi and Fortin 1991). The resulting nonlinear system of algebraic equations is expected to be a sufficient approximation of the original problem.

A nonuniform quadrangular mesh is considered as the spatial discretization for reservoir and a uniform linear mesh is considered as the spatial discretization for well annulus and tubing as shown in Figure 4.12. 

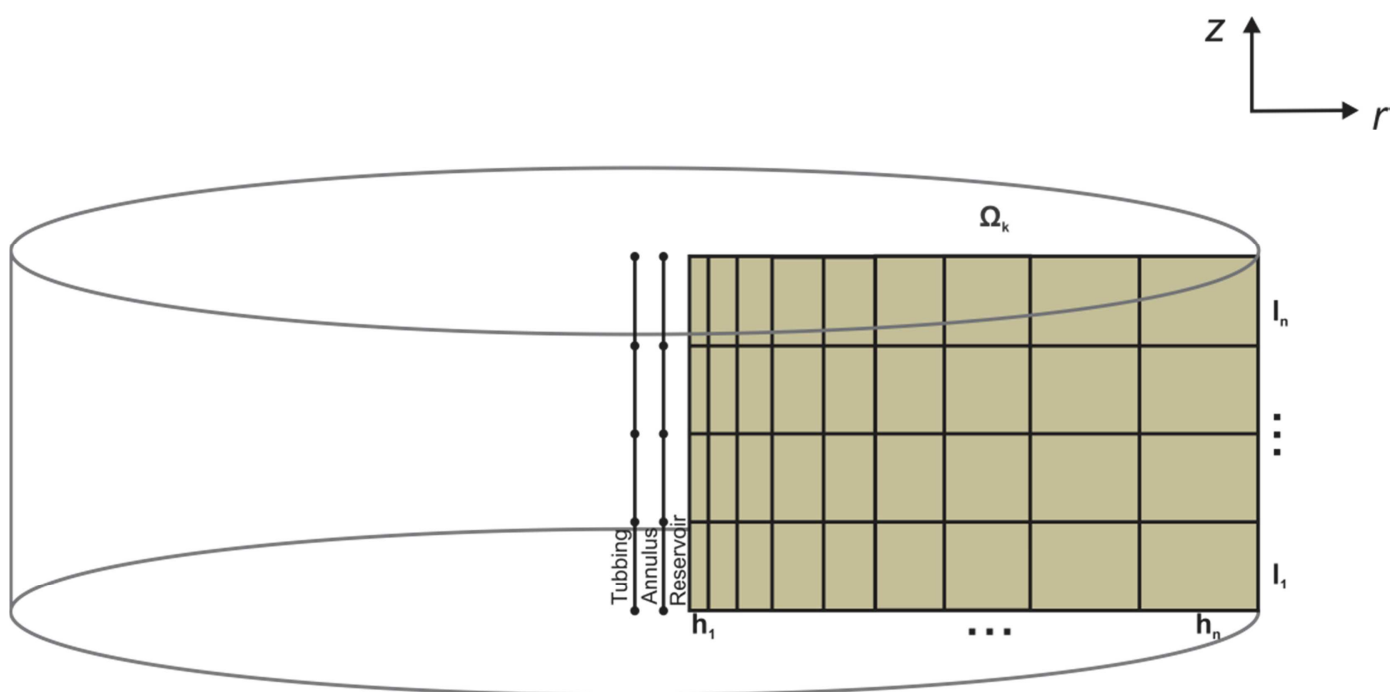

Figure 4.12 - Nonuniform quadrangular mesh.

An exponential element size function (4.21) was used to minimize numerical error in $r$ direction due to expected instability close to the well-reservoir interface where two dimensional and one dimensional element are coupled.

$$
h_{i}=h_{1}\left(\left(\frac{h_{N}}{h_{1}}\right)^{\frac{1}{(N-1)}}\right)^{i},
$$

Where

$h_{1}$ and $h_{N}$ are the first and the last element size respectively.

The nonlinear system of equations can be explicitly rewritten in residual form as:

In the reservoir:

$$
\begin{aligned}
R_{r}{ }^{p}= & \phi c_{t} \int_{\Omega_{h}} \hat{p} \frac{\partial p}{\partial t} d v_{h}-\lambda_{t} \int_{\Omega_{h}}\left(\mathrm{k}_{r} \cdot \frac{\partial \hat{p}}{\partial \mathrm{r}} \frac{\partial p}{\partial r}+\mathrm{k}_{z} \cdot \frac{\partial \hat{p}}{\partial z}\left(\frac{\partial p}{\partial z}-\rho_{t} g \sin \theta\right)\right) d v_{h}+ \\
& +\lambda_{t} \int_{\partial \Omega_{h}}\left(k_{r} \cdot \hat{p} \frac{\partial p}{\partial r}+k_{z} \cdot \hat{p}\left(\frac{\partial p}{\partial z}-\rho_{t} g \sin \theta\right)\right) d a_{h} \\
R_{r}^{T}= & \left(\rho C_{p}\right)_{t} \int_{\Omega_{h}} T \frac{\partial T}{\partial t} d v_{h}-\phi(\beta)_{t} \int_{\Omega_{h}} T T \frac{\partial p}{\partial t} d v_{h}- \\
& -\phi c_{f} \int_{\Omega_{h}} T\left(p+\rho_{f} C_{p f} T\right) \frac{\partial p}{\partial t} d v_{h}+ \\
& +\left(\rho C_{p}\right) \int_{\Omega_{h}} \hat{T} \lambda_{t}\left(k_{r} \frac{\partial T}{\partial r} \frac{\partial p}{\partial r}+k_{z} \frac{\partial T}{\partial z}\left(\frac{\partial p}{\partial z}-\rho_{t} g \sin \theta\right)\right) d v_{h}+
\end{aligned}
$$




$$
\begin{aligned}
& +\int_{\Omega_{h}}(1-(\beta) T) \hat{T} \lambda_{t}\left(k_{r}\left(\frac{\partial p}{\partial r}\right)^{2}+k_{z} \frac{\partial p}{\partial z}\left(\frac{\partial p}{\partial z}-\rho_{t} g \sin \theta\right)\right) d v_{h}- \\
& -\int_{\Omega_{h}}\left(k_{T r} \frac{\partial T}{\partial r} \frac{\partial T}{\partial r}+k_{T z} \frac{\partial T}{\partial r} \frac{\partial T}{\partial z}\right) d v_{h}+\int_{\partial \Omega_{h}}\left(k_{T r} T \frac{\partial T}{\partial r}+k_{T z} T \frac{\partial T}{\partial z}\right) d a_{h}
\end{aligned}
$$

In the well annulus:

$$
\begin{aligned}
R_{w}{ }^{u_{a}}= & A_{a} \int_{\Omega_{h}} \hat{u}_{a} \frac{\partial(\rho y u)_{a}}{\partial z} d z_{h}-2 \pi R_{c i} \int_{\Omega_{h}} \hat{u}_{a}(\gamma \rho y u)_{I} d z_{h} \\
R_{w}{ }^{p_{a}}= & \int_{\Omega_{h}} \hat{p}_{a} \frac{\partial p_{a}}{\partial z} d z_{h}-\int_{\Omega_{h}} \hat{p}_{a}\left(\frac{\partial \rho_{a} u_{a}^{2}}{\partial z}+\frac{2 f_{F a} u_{a}^{2} \rho_{a}}{D_{h a}}+\rho_{a} g \sin \theta\right) d z_{h} \\
R_{w}{ }^{T_{a}}= & A_{a} \int_{\Omega_{h}}\left(\rho y u C_{p}\right)_{a} \hat{T}_{a} \frac{\partial T_{a}}{\partial z} d z_{h}- \\
& -\int_{\Omega_{h}} \hat{T}_{a}\left(2 \pi R_{c i}(1-\gamma) U_{a f}\left(T_{f}-T_{a}\right)-2 \pi R_{t i} U_{t a}\left(T_{a}-T_{t}\right)\right) d z_{h}- \\
& -\int_{\Omega_{h}} \hat{T}_{a}\left(2 \pi R_{c i}\left(\gamma \rho y u C_{p}\left(T_{I}-T_{a}\right)\right)_{I}+A_{a}(\rho y u)_{a} g \sin \theta\right) d z_{h}- \\
& -A_{a} \int_{\Omega_{h}} \hat{T}_{a}\left(\left(\rho y u C_{p} k_{J T}\right)_{a} \frac{\partial p_{a}}{\partial z}+\left(\rho y u C_{p}\right)_{a} h_{l a t, g o}\left(\left.\frac{\rho_{a g}}{\rho_{a o}} \frac{\partial R_{s}}{\partial p_{a}}\right|_{T_{a}}\right) \frac{\partial p_{a}}{\partial z}\right) d z_{h}
\end{aligned}
$$

In the well tubing:

$$
\begin{aligned}
R_{w}^{u_{t}}= & A_{t} \int_{\Omega_{h}} \hat{u}_{t} \frac{\partial(\rho y u)_{t}}{\partial z} d z_{h} \\
R_{w}{ }^{p_{t}}= & \int_{\Omega_{h}} \hat{p}_{t} \frac{\partial p_{t}}{\partial z} d z_{h}-\int_{\Omega_{h}} \hat{p}_{t}\left(\frac{\partial \rho_{t} u_{t}^{2}}{\partial z}+\frac{2 f_{F a} u_{t}^{2} \rho_{t}}{D_{t}}+\rho_{t} g \sin \theta\right) d z_{h} \\
R_{w}^{T_{t}}= & A_{t} \int_{\Omega_{h}}\left(\rho y u C_{p}\right)_{t} \hat{T}_{t} \frac{\partial T_{t}}{\partial z} d z_{h}- \\
& -\int_{\Omega_{h}} \hat{T}_{t}\left(2 \pi R_{t i} U_{t a}\left(T_{a}-T_{t}\right)+A_{t}(\rho y u)_{a} g \sin \theta\right) d z_{h}- \\
& -A_{t} \int_{\Omega_{h}} \hat{T}_{t}\left(\left(\rho y u C_{p} k_{J T}\right)_{t} \frac{\partial p_{t}}{\partial z}+\left(\rho y u C_{p}\right)_{t} h_{l a t, g o}\left(\left.\frac{\rho_{t g}}{\rho_{t o}} \frac{\partial R_{s}}{\partial p_{t}}\right|_{T_{t}}\right) \frac{\partial p_{t}}{\partial z}\right) d z_{h}
\end{aligned}
$$

Limited to the domain $\Omega_{\mathrm{h}}$. 
The resulting system remains time dependent. Implicit time integration methods find the solution using both current solution and later time unknowns. Hence, implicit Euler method was chosen to perform time integration:

Let $f^{k}$ be the current unknowns and $f^{k+1}$ the unknowns in a later time so for the implicit Euler we can write:

$$
\frac{\partial f}{\partial t}=g(k, f) \rightarrow \frac{f^{k+1}-f^{k}}{\delta t}=g\left(t, f^{k+1}\right) \rightarrow f^{k}=f^{k+1}-g\left(t, f^{k+1}\right) \delta t
$$

This equation shows that the system of equations must be solved in order to get the values of the unknowns in the later time $f^{k+1}$. The implicit time fully coupled system from the well-reservoir equations is solved using Newton's method (Kelley 2003). It is a powerful iterative technique despite of its small radius of convergence which requires a good initial guess. A simple linear extrapolation was chosen to achieve this requirement:

$$
c^{k+1}=2 c^{k}-c^{k-1}
$$

The well-reservoir nonlinear system of equations (4.22):

$$
R(c)=0,
$$

is solved from its associated Jacobian matrix and a residue vector through a sequence of iterations of the linear system:

$$
\begin{aligned}
& J\left(\boldsymbol{c}^{k}\right) \delta \boldsymbol{c}^{k}=-R\left(\boldsymbol{c}^{k}\right) \\
& \boldsymbol{c}^{k+1}=\boldsymbol{c}^{k}+\delta \boldsymbol{c}^{k}
\end{aligned}
$$

this is continued until $R\left(\boldsymbol{c}^{k}\right)<\operatorname{tol}_{\text {res }} R\left(\boldsymbol{c}^{0}\right)$ or $\delta \boldsymbol{c}^{k}<\operatorname{tol}_{\text {update }} \boldsymbol{c}^{k}$.

Where

$$
J_{i, j}\left(c^{k}\right)=\frac{\partial R_{i}}{\partial c^{k}},
$$

is the Jacobian matrix elements and $t o l$ is the required tolerance for the chosen stopping method. Due to its flexibility a numerical Jacobian matrix was used instead of an analytic one even at the expense of performance and the possibility of a small numerical error. The numerical Jacobian is computed using the following steps: 


$$
\boldsymbol{R}\left(\boldsymbol{c}^{1}\right)
$$

for $j$ from 1 to \# columnsdo

$$
\begin{aligned}
& \boldsymbol{c}_{p}{ }^{j} \leftarrow \boldsymbol{c}^{j}+\delta \\
& J_{\# \text { rows }, j}\left(\boldsymbol{c}^{j}\right) \leftarrow \frac{\boldsymbol{R}\left(\boldsymbol{c}_{p}{ }^{j}\right)-\boldsymbol{R}\left(\boldsymbol{c}^{j}\right)}{\delta} \\
& \boldsymbol{c}_{\boldsymbol{p}}{ }^{j} \leftarrow \boldsymbol{c}^{j}
\end{aligned}
$$

end for

The resulting sparse linear system is solved using a preconditioned GMRES iterative algorithm. Approximate minimum degree reordering was chosen as the preconditioner. 


\section{5}

\section{Validation and Case Studies}

\section{1}

\section{Segment Validation}

The models for one reservoir layer and one well segment were validated against a commercial multiphysics modeling and simulation tool $\left(\mathrm{Comsol}^{\mathrm{TM}}\right)$ and a dynamic multiphase flow simulator $\left(\mathrm{Olga}^{\mathrm{TM}}\right)$ independently and without valves. Afterwards a fully coupled simulation for the well-reservoir layer/segment is compared with Figure 4.5. The configuration used in the model for one layer/segment is shown in Figure 5.1. It is used in the validation procedure (without ICVs) and case studies (with ICVs).

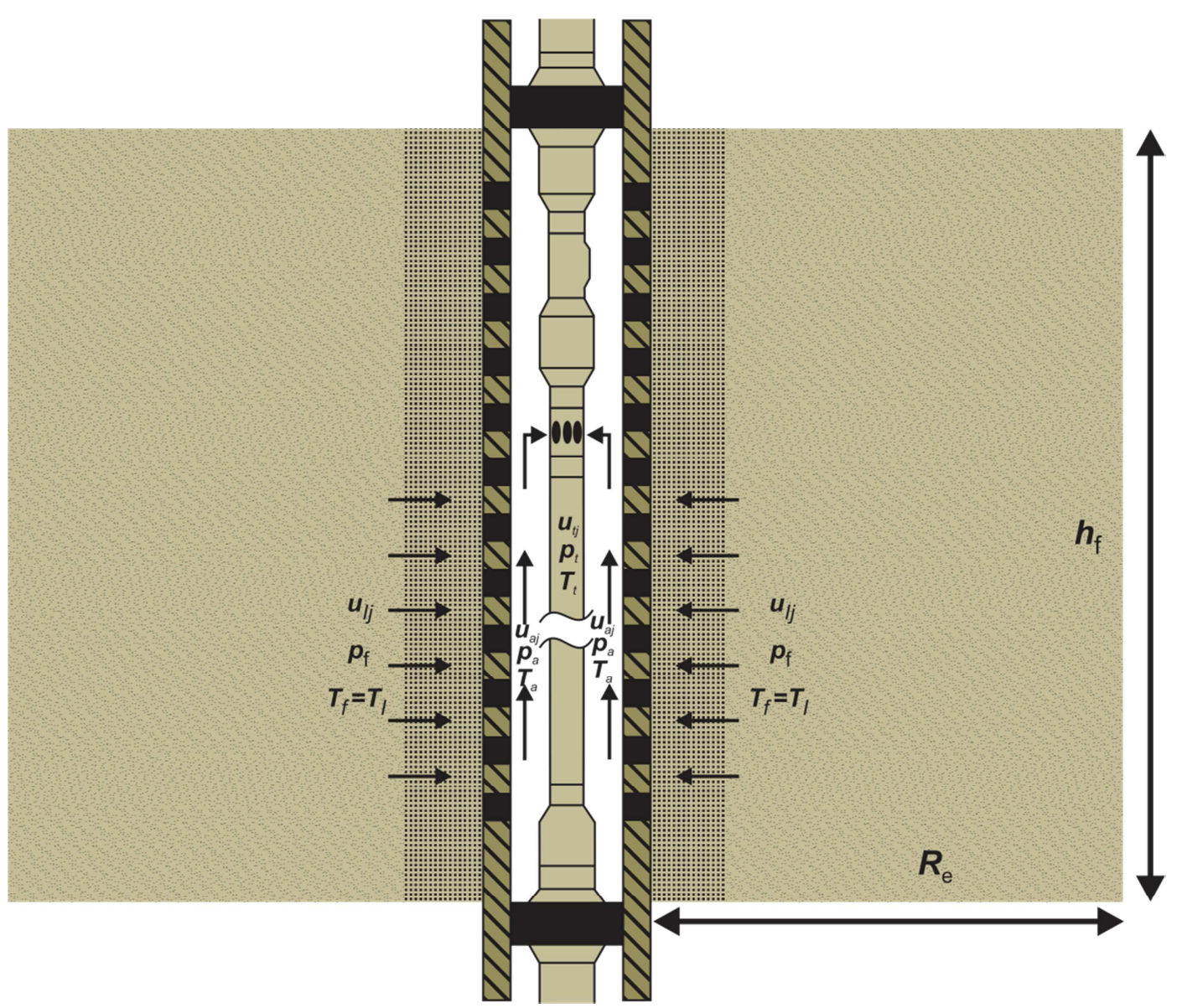

Figure 5.1 - One well-reservoir layer/segment with an ICV. 


\subsection{1 Vertical Well}

The well segment experiencing steady state flow of 500 bpd of oil production in the tubing and in the annulus at the top of the segment is simulated for pressure and temperature. The simulation results are presented as: $p_{a}, p_{t}, T_{a}$ and $T_{t}$ profiles which are validated against Olga $^{\mathrm{TM}}$ using data reported in (Muradov 2010) and reproduced as square dots in the Figure 5.2. As expected, pressure profiles are dominated by hydraulic head and a uniform influx from the annulus. Temperature profiles clearly show differences for tubing and annulus and a good agreement with the reference data. The quality measures is reported as the Euclidean Norm of the relative error which is $8.910^{-4}$ for $T_{a}$ and $1.910^{-3}$ for $T_{t}$.
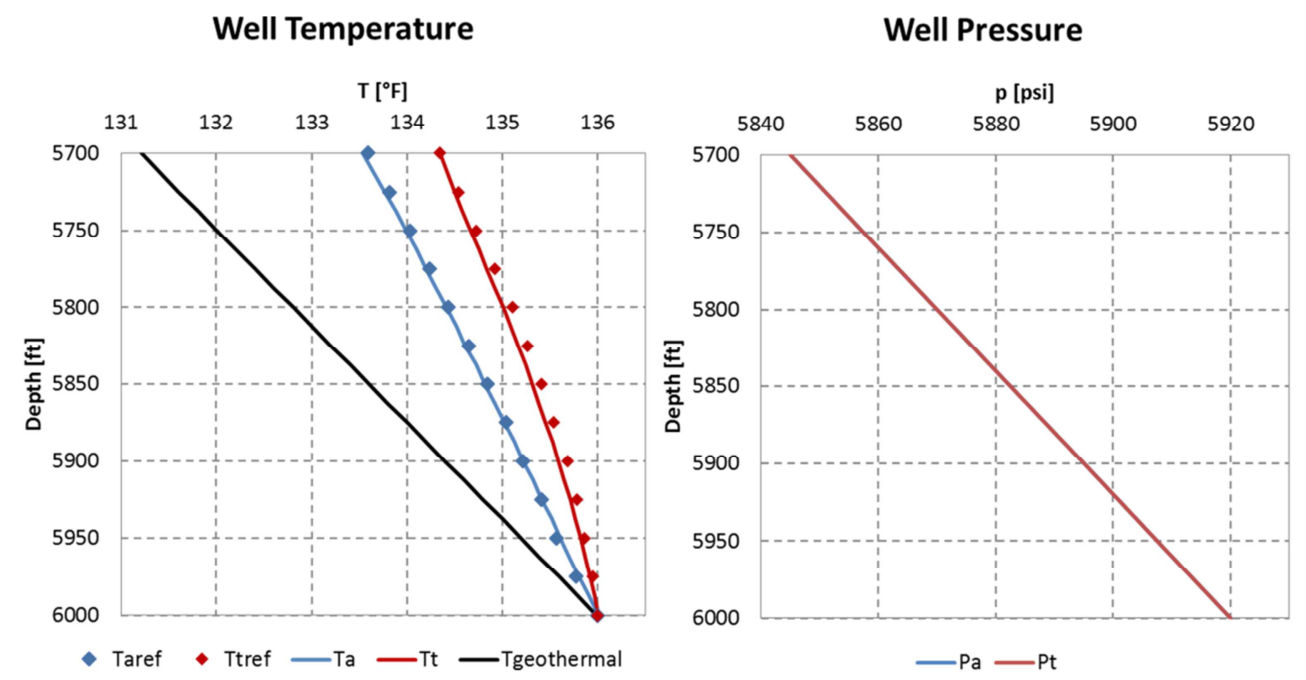

Figure 5.2 - Temperature and pressure in a vertical well with 500 bpd of oil production in the tubing and in the annulus.

The same simulation is repeated for $500 \mathrm{bpd}$ of oil/water in the tubing and in the annulus at the top of the segment. The results are validated against $\mathrm{Olga}^{\mathrm{TM}}$ giving the Euclidean Norm of the relative error $1.610^{-4}$ for $T_{a}$ and $6.210^{-3}$ for $T_{t}$ for this case. 

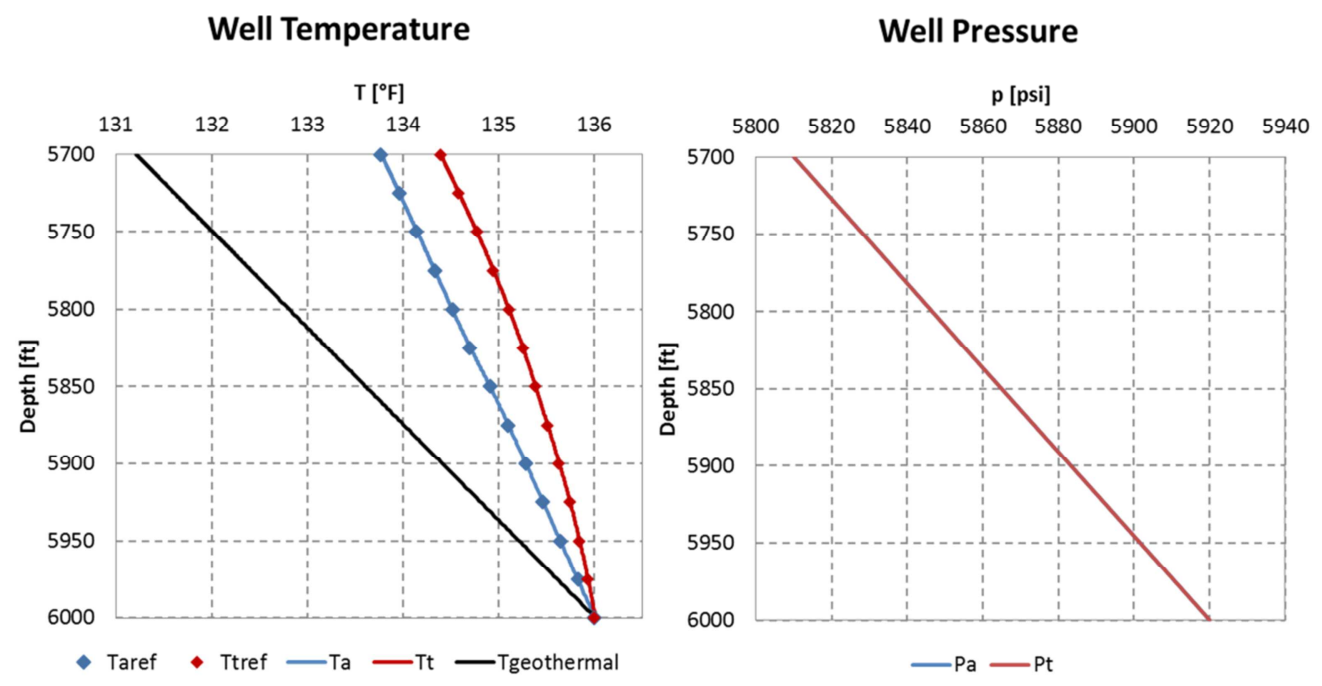

Figure 5.3 - Temperature and pressure in a vertical well with $500 \mathrm{bpd}$ of oil/water production in the tubing and in the annulus.

\subsection{2}

\section{Cylindrical shaped reservoir}

The cylindrical shaped reservoir is simulated for oil and oil/water up to 48 hours of production time after a step rate change of 2000 [bpd] of the total flow done at the surface. During the simulations $10 \%$ of leak is allowed in $\mathrm{z}$ direction and gravitational effect and geothermal gradient are considered. The parameters used for the simulation are presented in Table 5.1 except by reservoir thickness: $150[\mathrm{ft}]$, reference temperature: $154.8\left[{ }^{\circ} \mathrm{F}\right]$, equivalent radius: $1000[\mathrm{ft}]$, and saturation: $S_{o}=1$ and $S_{o}=0.5$ respectively to run the simulations. A commercial multiphysics modeling and simulation tool $\left(\mathrm{Comsol}^{\mathrm{TM}}\right)$ is used as a reference tool for: mesh size and time step tuning. The best match after the tuning procedure is shown in Figures 5.3 and 5.4 for both cases. It was achieved using a minimum time step of 6 minutes and mesh size 45/45 with density variation over $r$ direction calculated by equation 4.21 using $h_{45} / h_{l}=1 / 15$. The quality measure was again based on the Euclidean norm of the relative error which were for oil production: $5.610^{-3}$ for $p\left(r=R_{w b}, t\right), 5.310^{-5}$ for $p(r, t=48 h), 1.110^{-3}$ for $T\left(r=R_{w b}, t\right)$, and $1.510^{-5}$ for $T(r, t=48 h)$. 

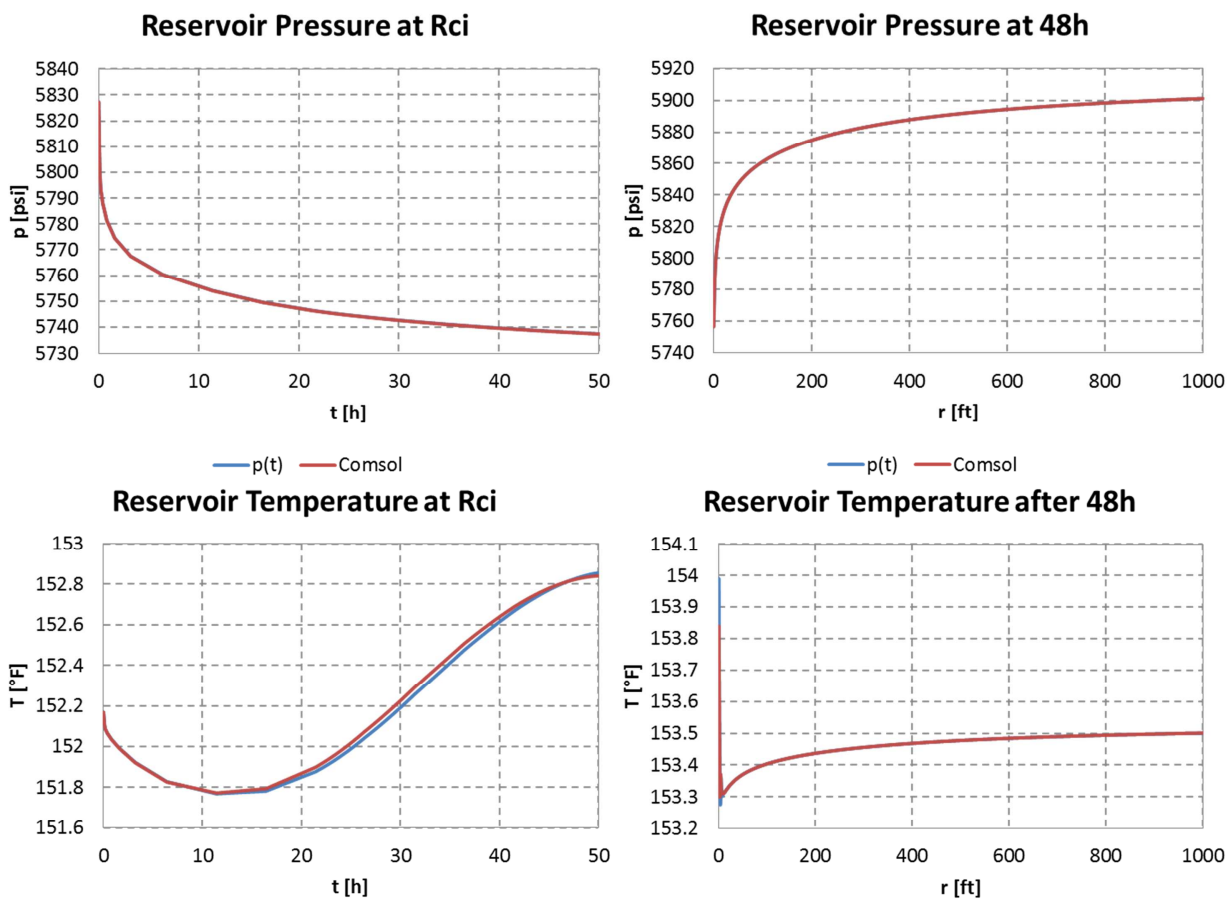

- $T(\mathrm{t})$ - Comsol

$-T(r)-$ Comsol

Figure 5.3 - Reservoir pressure and temperature (oil production)

In the case of oil/water production the Euclidean norm of the relative error were: $3.410^{-4}$ for $p\left(r=R_{w b}, t\right), 7.510^{-3}$ for $p(r, t=24 h), 4.210^{-4}$ for $T\left(r=R_{w b}, t\right)$, and $5.910^{-4}$ for $T(r, t=24 h)$.
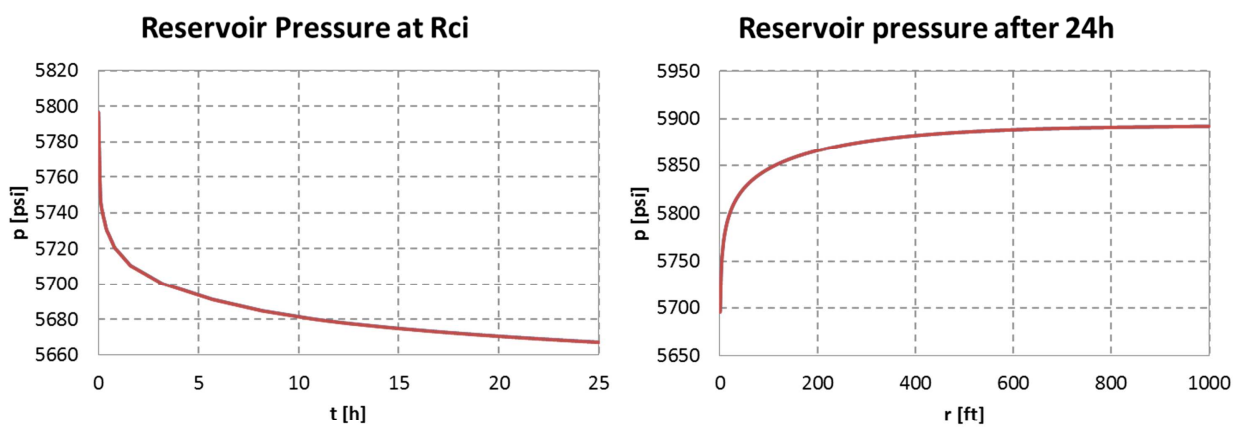

- $p(t)$ Comsol

- $p(r)$ Comsol
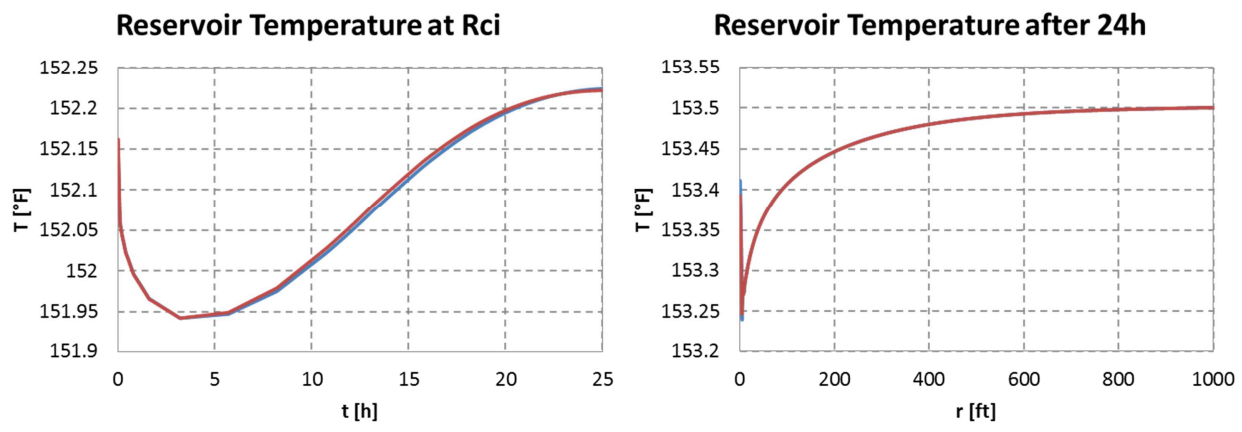

- $T(\mathrm{t})$ - Comsol

- $T(r)$ Comsol

Figure 5.4 - Reservoir pressure and temperature (oil/water production) 
The validation procedure is completed through the fully coupled simulation of the segment for oil/water production experiencing two transients: first one 2000 [bdp] and second one 1000 [bpd] both are choke openings. A comparison of the pressure and temperature behavior illustrated in Figure 4.5 indicates good agreement with the real data, as shown in Figure 5.5.

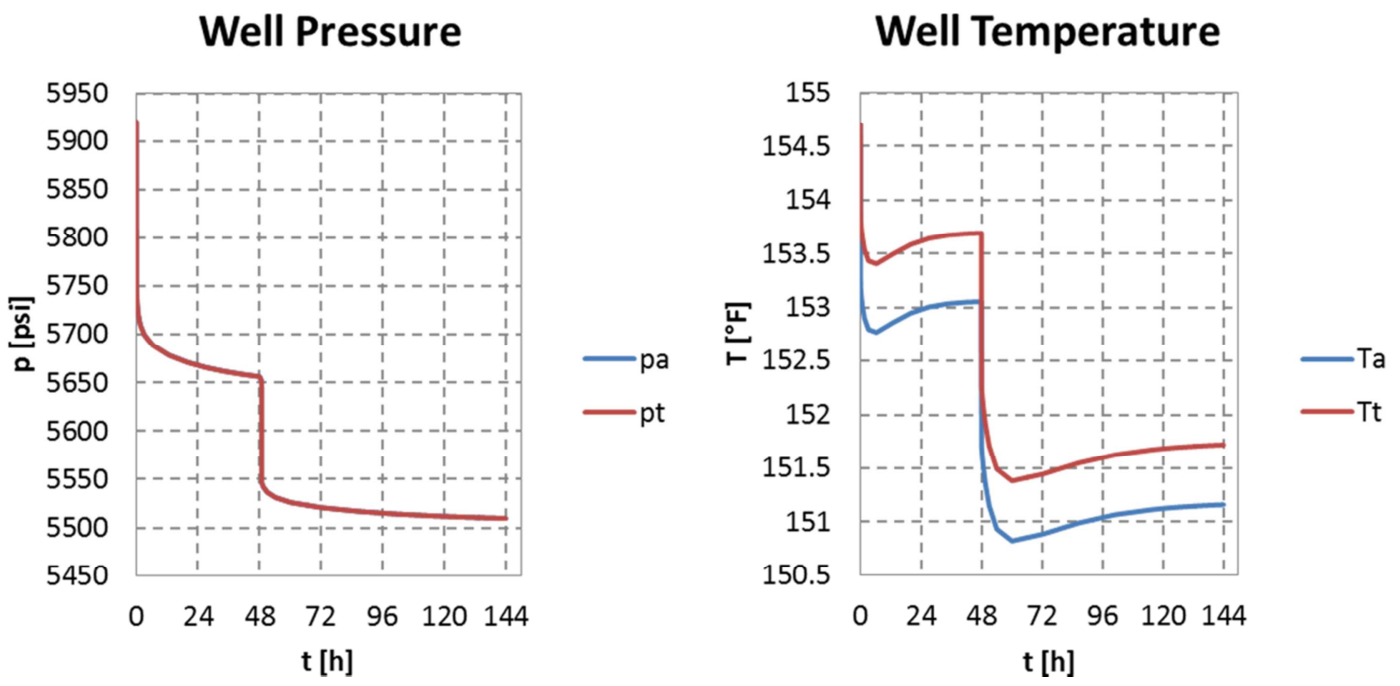

Figure 5.5 - Pressure and temperature transients for 2000 [bpd] and 1000 [bpd].

\section{1 .3 \\ Case Studies}

The model derived captures transient behavior in a multilayer vertical well equipped with intelligent completion. Basically, it is a reasonable example of an IW application. In an intelligent field scenario, real-time analysis tools are used for reservoir management leading to production optimization, better reservoir characterization and flow allocation. Through the use of appropriate tools it is possible, in theory, to do pressure and temperature transient analysis without intervention or well shut-in. Automatic well tests were applied in a valid set of continuously measured pressure during unplanned shut-in periods proving that it is possible (Olsen and Nordtvedt 2006).

Well-testing uses generally pressure transient responses due to production rate changes to gather information about reservoir properties. Temperature transients can also be used for this purposes as indicated by (Sui, EhligEconomides et al. 2010) for conventional wells and by (Muradov and Davies 2010) for horizontal IWs. Therefore, it is possible in principle to have the same 
result when changing flowrate downhole by a multiposition ICV without zonal shut-in. The developed model is used to show qualitatively this possibility pushing a little bit further what have been developed which are only for zonal shut-in.

A three zone vertical IW equipped with multiposition ICVs is chosen as the study case. The choke profile is selected from a $3 \frac{1 / 2}{2}$ ICV of a commercial product line and it is the same in all the simulations. It is assumed constant properties per layer. Data used to perform the simulations is presented in Table 5.1 and a simplified well schematic in Figure 5.6. The pressure is assumed to be above the bubble point.

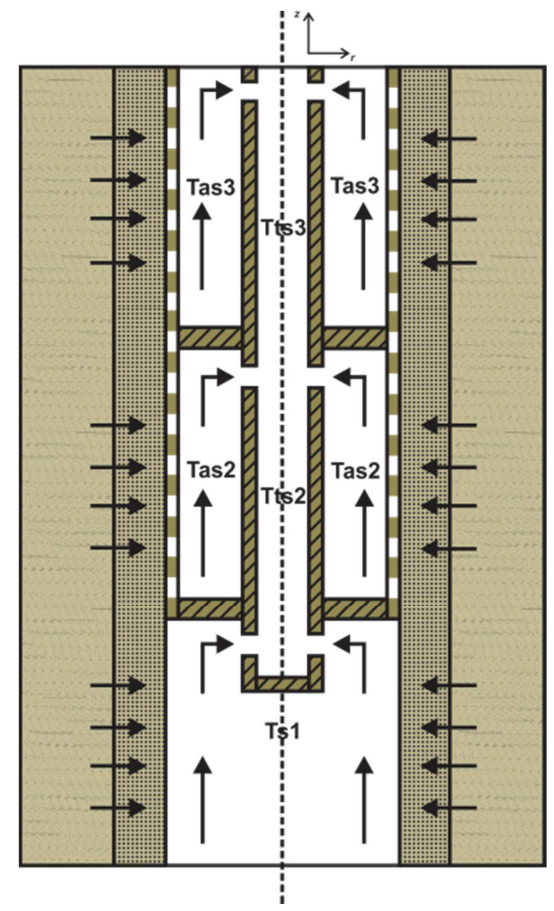

Figure 5.6 - Well-reservoir schematic for case study.

\subsubsection{1}

\section{Base Case}

The three zones are producing 1000 bpd of oil each with uniform flow distribution and the well choked. A transient of 3000 bpd is then created by opening the surface choke and all layers have to adapt to this new total rate of 6000 bpd. In addition, as all reservoir properties are equivalent at the different layers, the same behavior is expected towards to the new constant total flow rate. All ICVs are kept fully open during the whole numerical experiment. The result is 
shown in Figures 5.7 to 5.11. The temperature shifts show the expansion and Joule-Thomson effects in all zones. The valves and fluid mixture in the upper layers are also present. It should be noted that the presence of annulus and tubing in the model cannot be neglected for appropriate understanding of the temperature behavior.

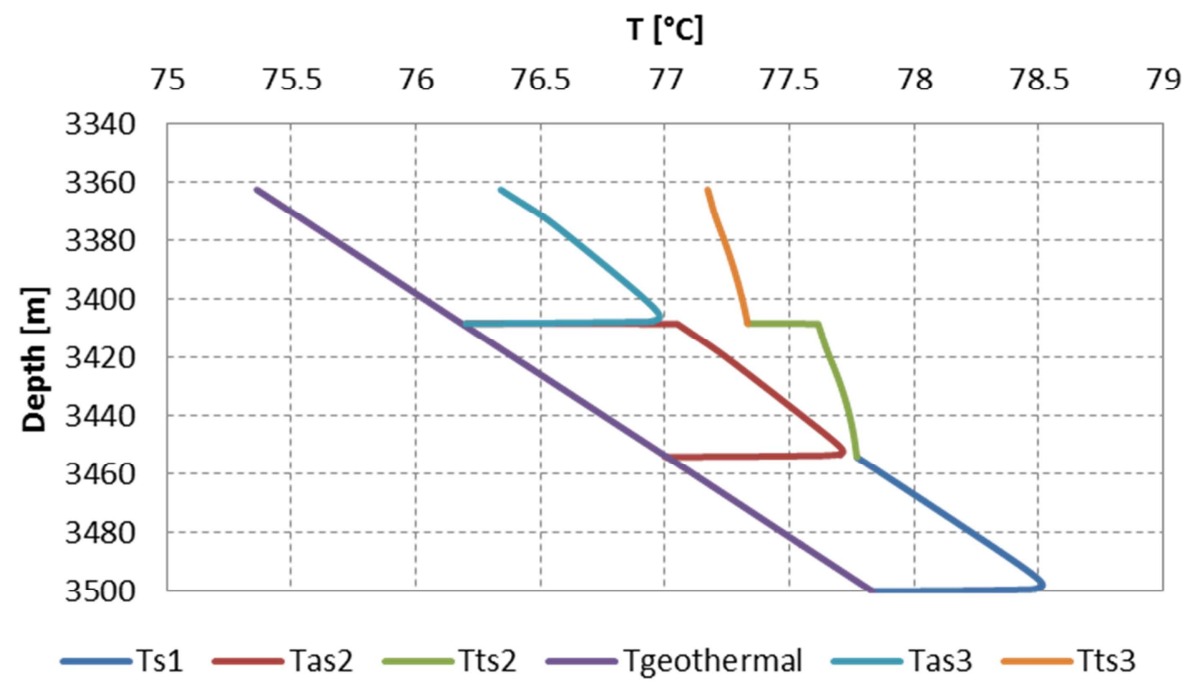

Figure 5.7 - Well temperature:T( $\mathrm{z}, \mathrm{t}=0)$.

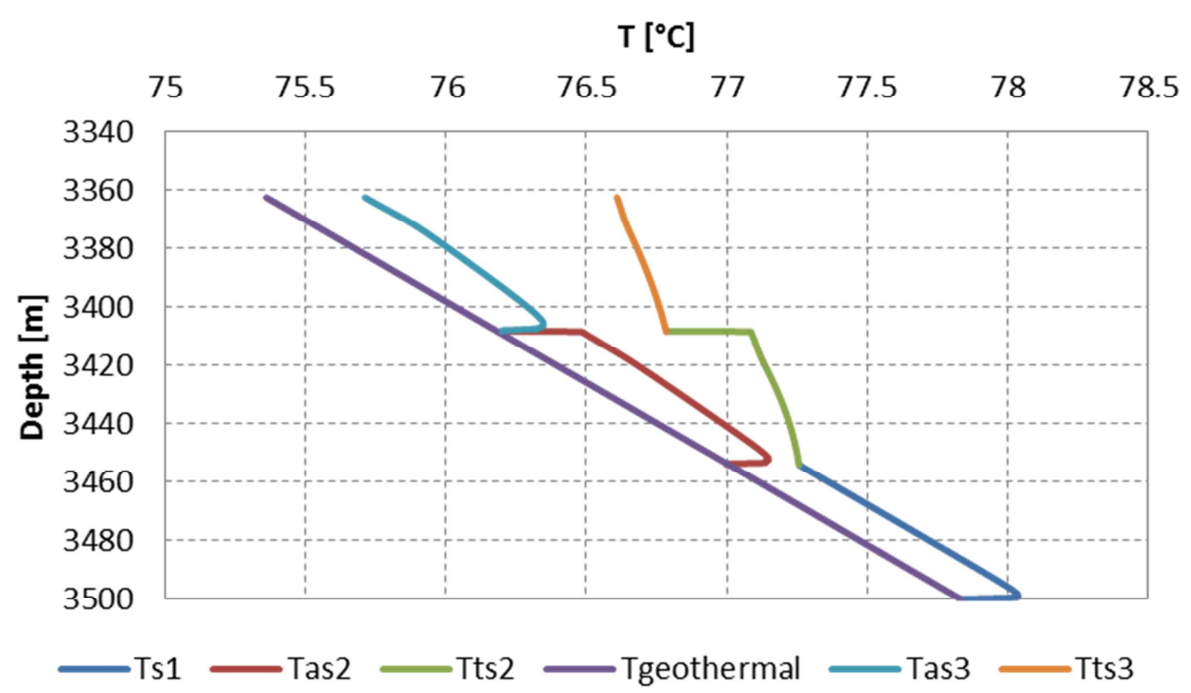

Figure 5.8 - Well temperature: $\mathrm{T}(\mathrm{z}, \mathrm{t}=3 \mathrm{~h})$. 


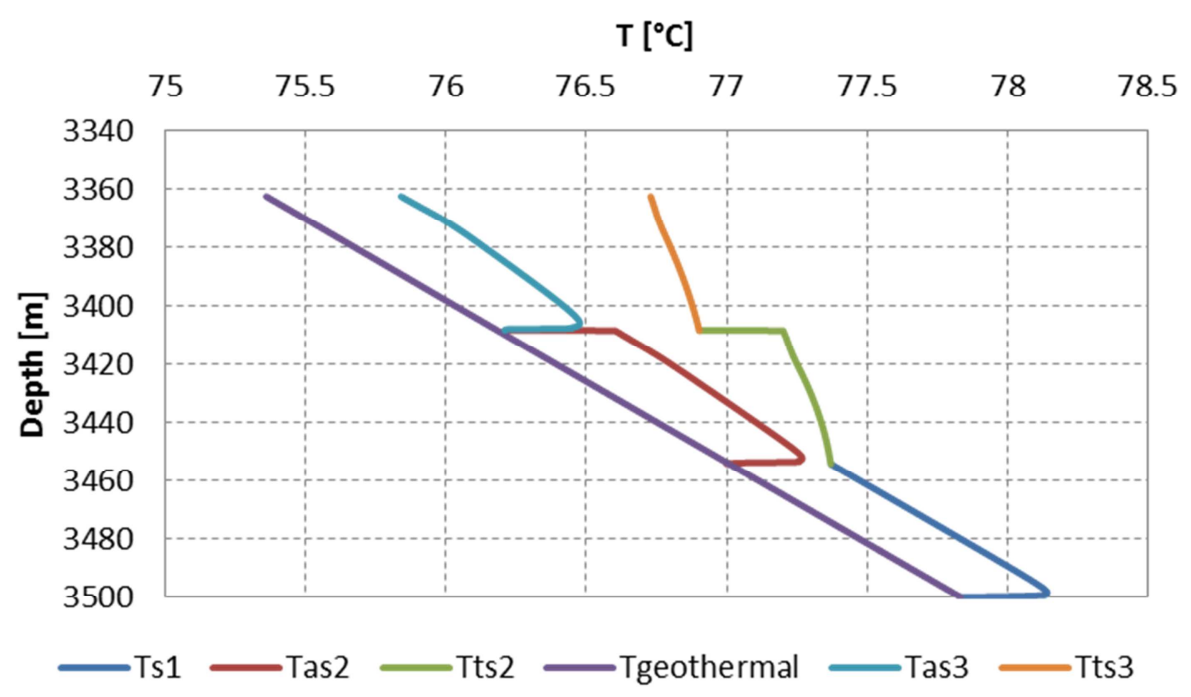

Figure 5.9 - Well temperature: $\mathrm{T}(\mathrm{z}, \mathrm{t}=24 \mathrm{~h})$.

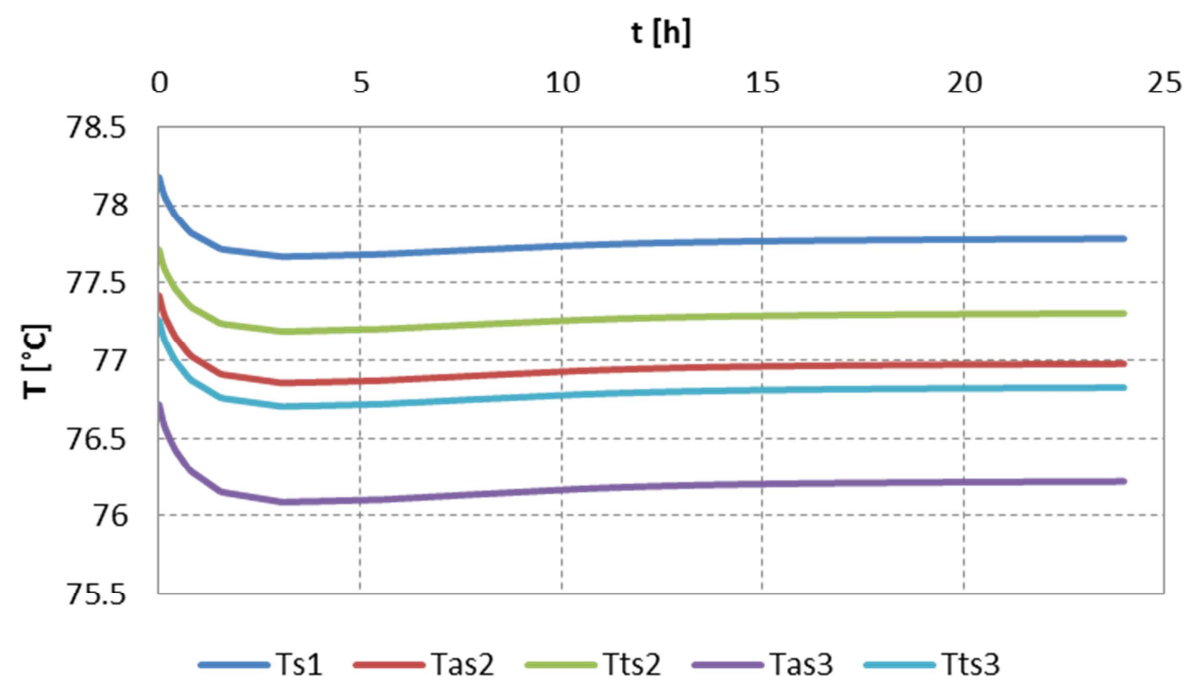

Figure 5.10 - Well temperature: $\mathrm{T}(\mathrm{z}=$ half segment, $\mathrm{t})$.

\subsubsection{2}

\section{Two-Phase at Middle Zone}

The second case study has the upper and lower zones producing $1000 \mathrm{bpd}$ of oil and the middle zone producing $50 \%$ of water and $50 \%$ of oil which is also producing $1000 \mathrm{bpd}$. A transient of $3000 \mathrm{bpd}$ is created by opening the surface choke and the total flowrate is kept constant as in the base case. The new fluid in the annulus of the middle layer has a stronger heat capacity with a weaker JouleThomson effect when compared with the fluid in the tubing. The heat cannot be 
transferred fast enough resulting in the different behavior of the temperature profiles and temperature transient presented in Figures 5.12 to 5.15.

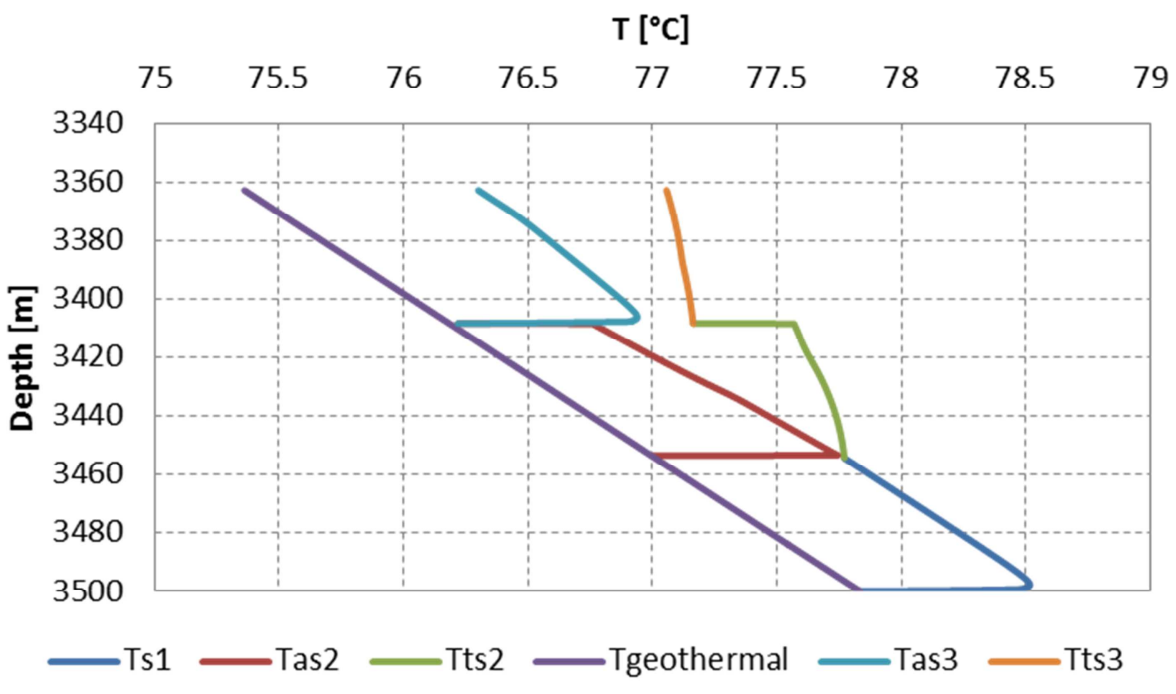

Figure 5.11 - Well temperature: $\mathrm{T}(\mathrm{z}, \mathrm{t}=0)$.

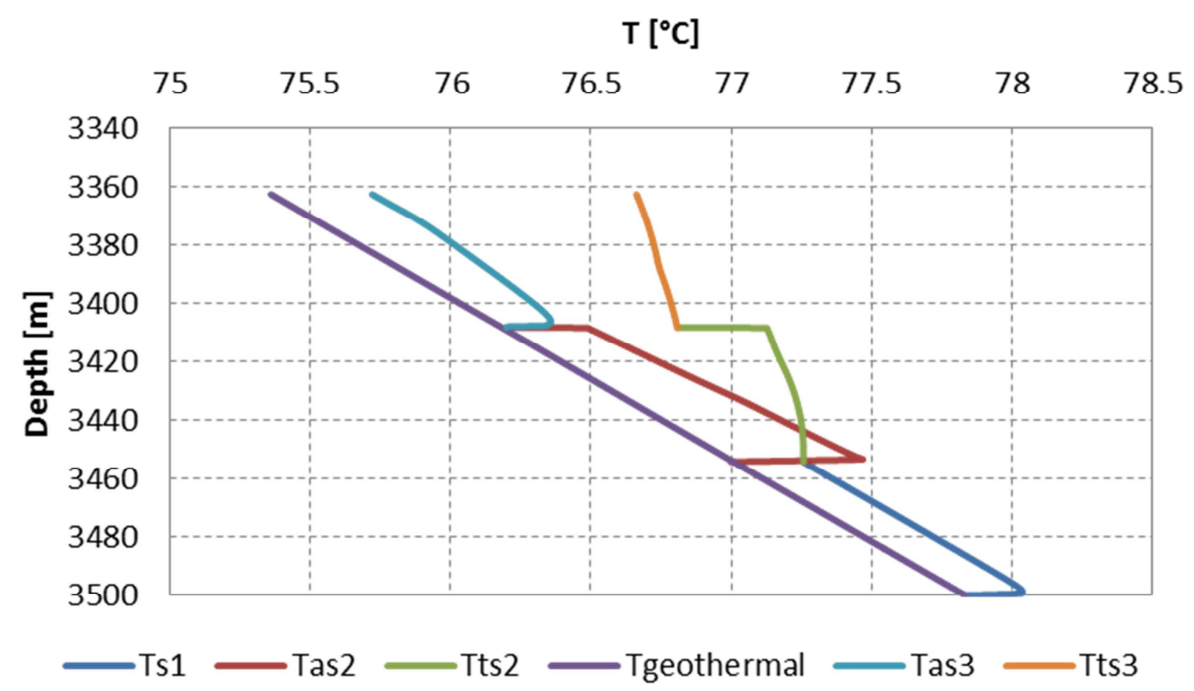

Figure 5.12 - Well temperature: $\mathrm{T}(\mathrm{z}, \mathrm{t}=3 \mathrm{~h})$. 


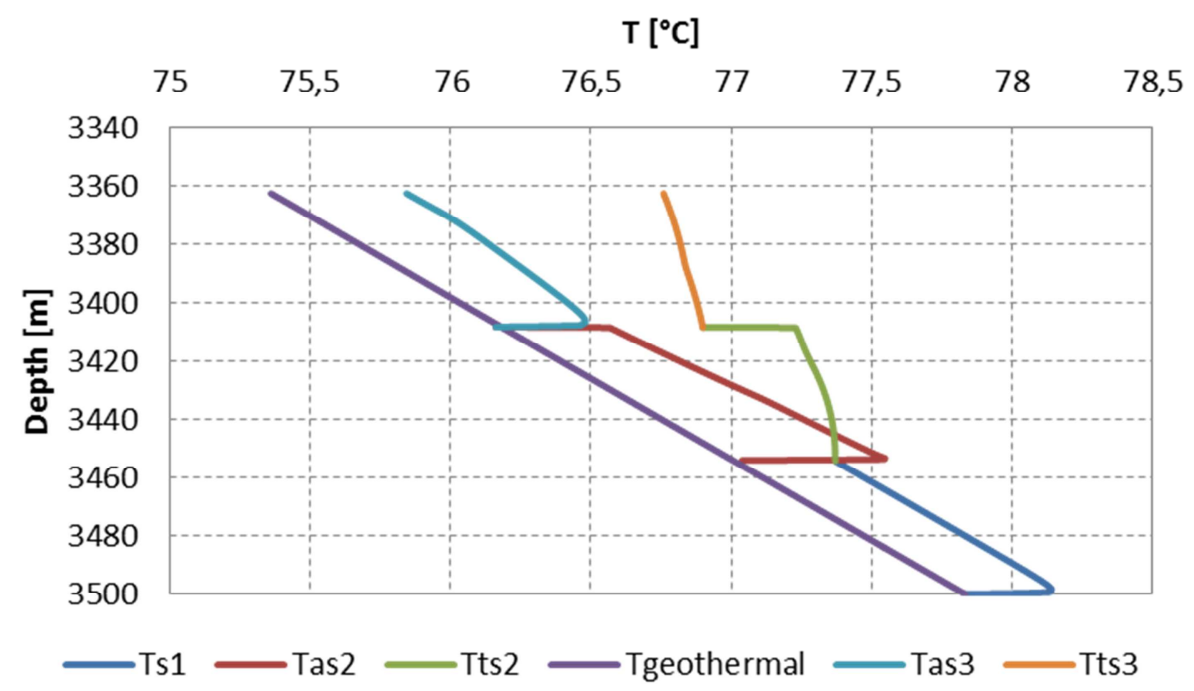

Figure 5.13 - Well temperature: $\mathrm{T}(\mathrm{z}, \mathrm{t}=24 \mathrm{~h})$.

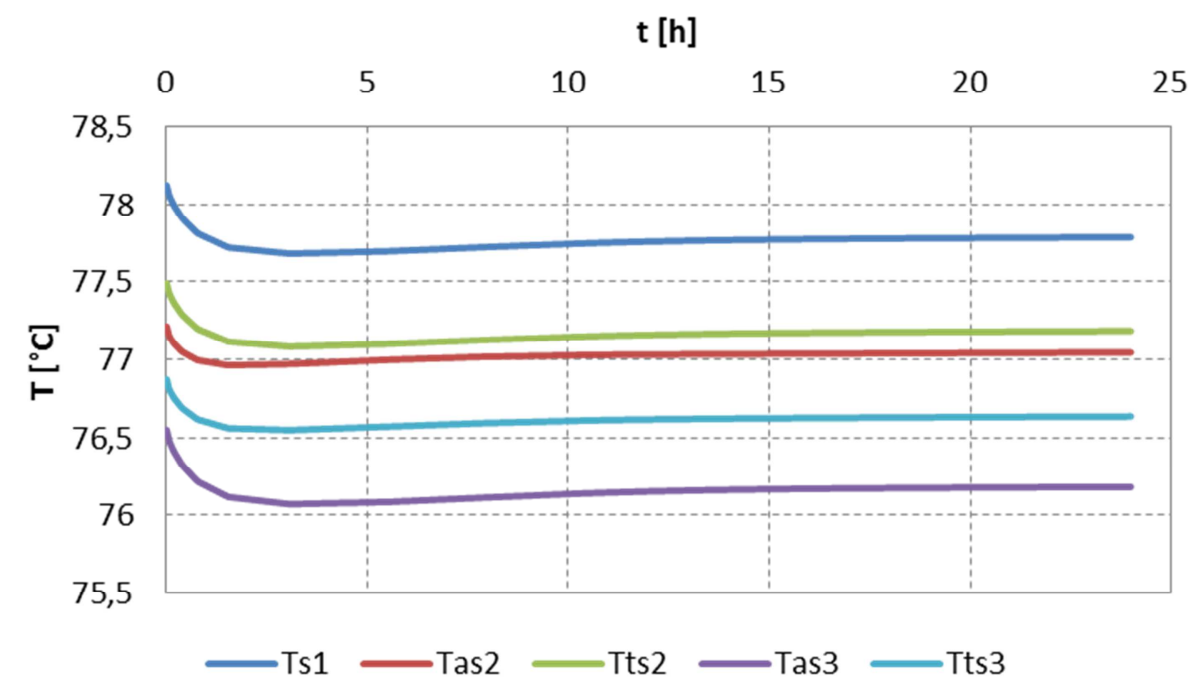

Figure 5.14 - Well temperature: $\mathrm{T}(\mathrm{z}=$ half segment, $\mathrm{t})$.

\subsubsection{3}

\section{Different Permeability at Middle Zone}

The third case study has a total flowrate of 3000 bpd but now the middle zone has a higher permeability. All zones are producing oil when a 3000 bpd transient is created by opening the surface choke. As in the base case the total flowrate of $6000 \mathrm{bpd}$ is kept constant. The temperature profiles and transient presented in Figures 5.16 to 5.18 show the effect of a higher flowrate at the middle layer resulting in a similar behavior of the previous case but weaker this 
time. It is also possible to note a stronger Joule-Thomson effect and a faster transient.

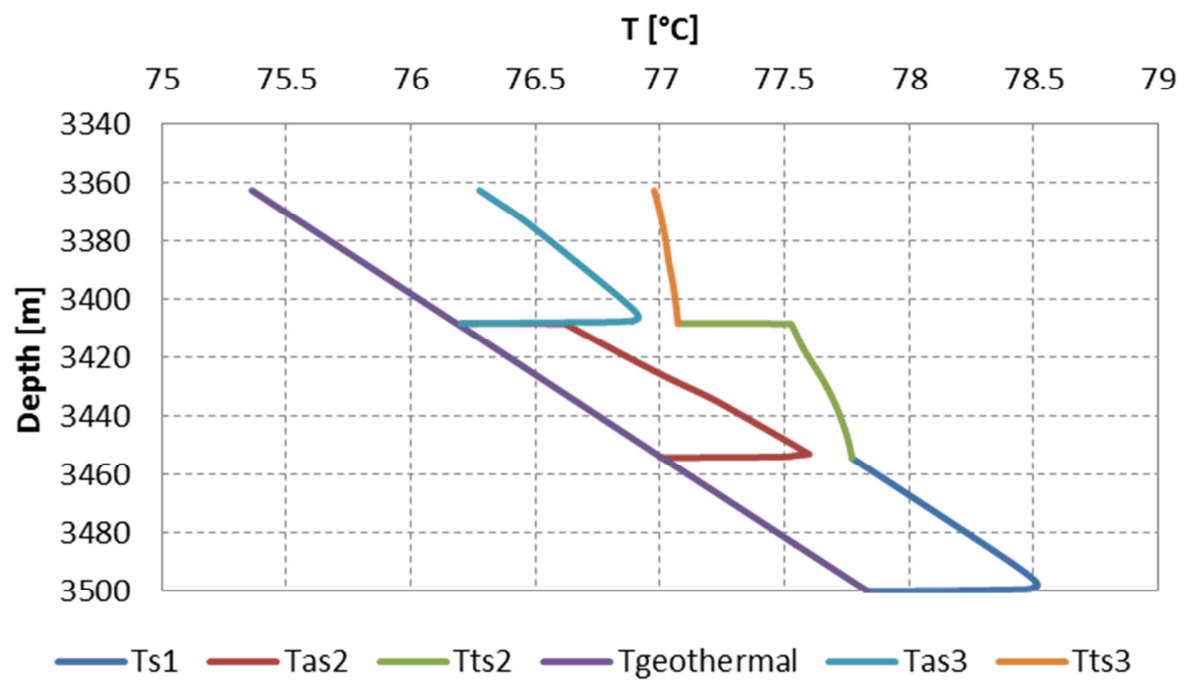

Figure 5.15 - Well temperature: $\mathrm{T}(\mathrm{z}, \mathrm{t}=0)$.

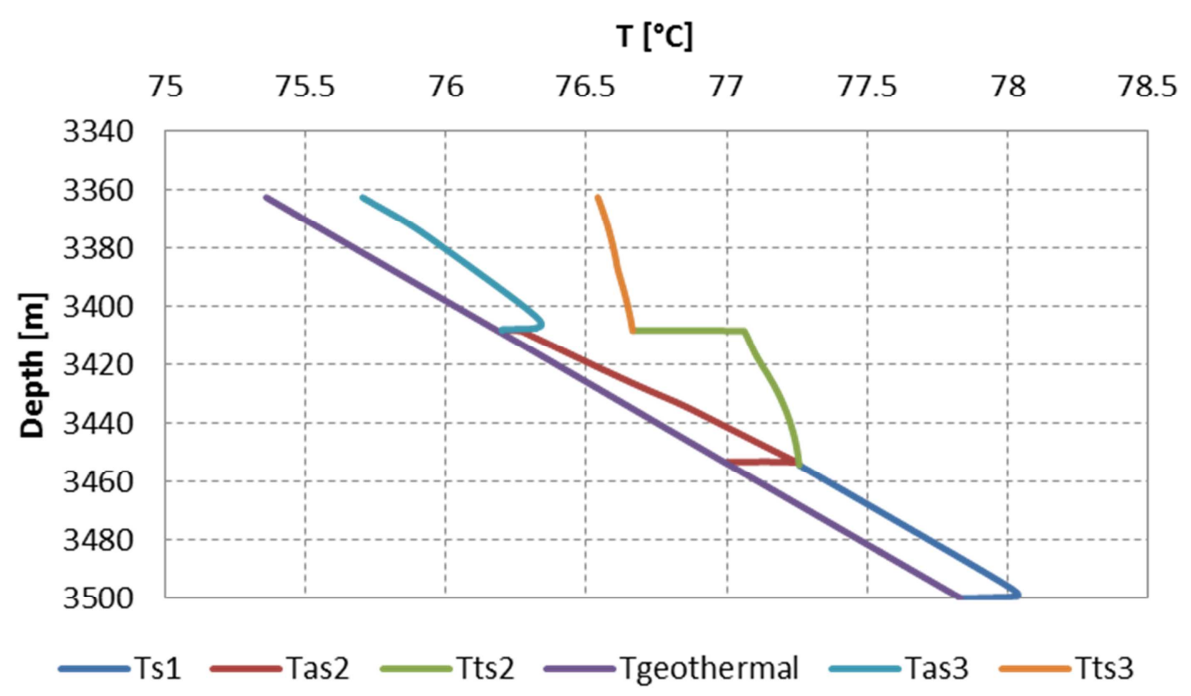

Figure- 5.16 - Well temperature:T(z, $t=3 h)$. 


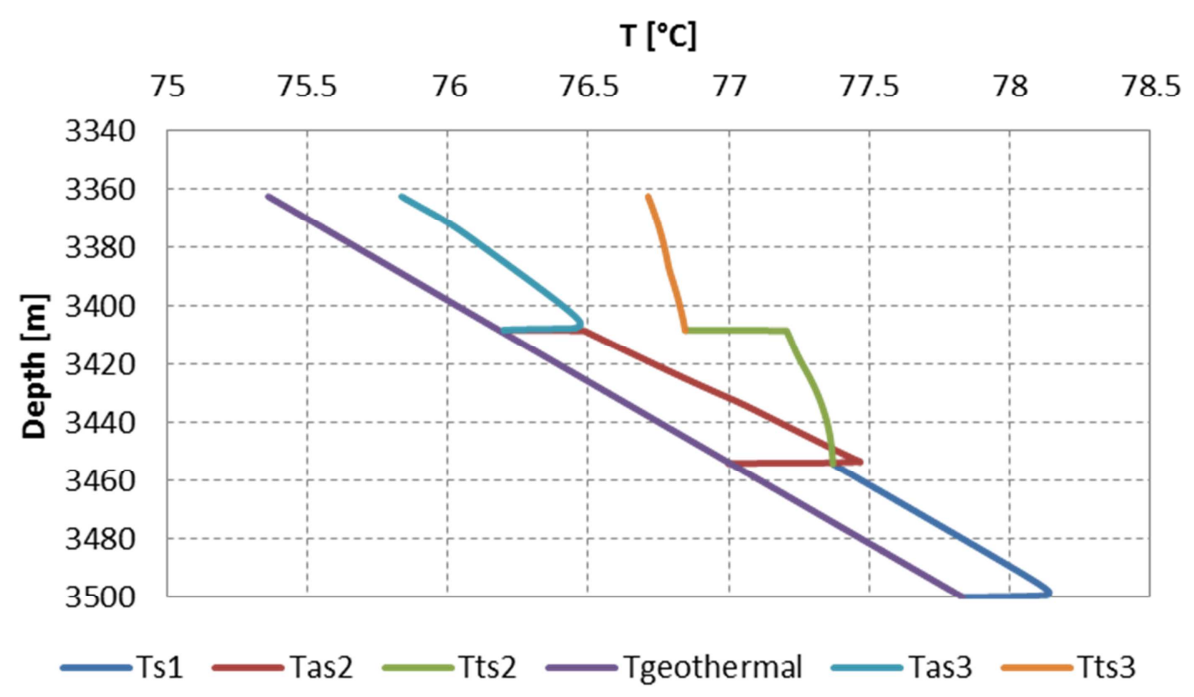

Figure 5.17 - Well temperature: $\mathrm{T}(\mathrm{z}, \mathrm{t}=24 \mathrm{~h})$.

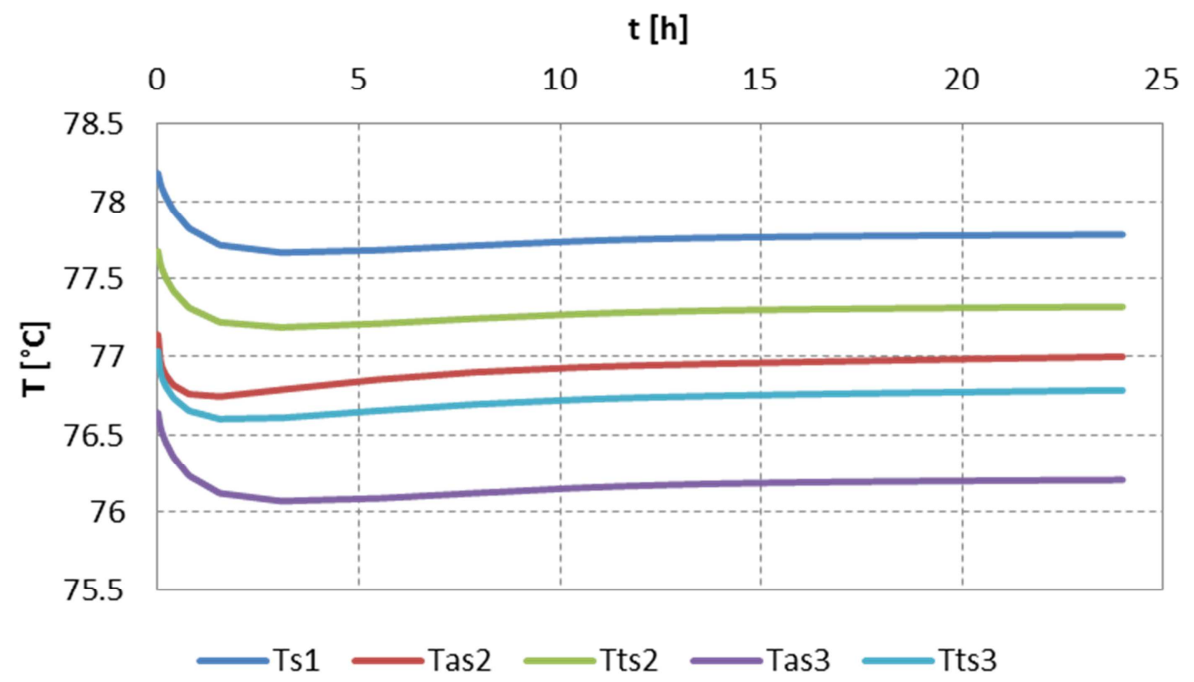

Figure 5.18 - Well temperature: $\mathrm{T}(\mathrm{z}=$ half segment, $\mathrm{t})$.

\subsubsection{4}

\section{Zonal Transient}

The last case study has a total flowrate of 5000 bpd which is kept constant during the experiment. Upper and lower zones are producing $1000 \mathrm{bpd}$ and the middle one $3000 \mathrm{bpd}$ of oil. A zonal transient of $2000 \mathrm{bpd}$ is created at the middle zone by changing the ICV flow area. The temperature profiles and transient reproduce the behavior found in a real well (Valiullin, Ramazanov et al. 2009), as presented in Figure 4.2. The temperature transient becomes weaker as the upper 
layers are approached and this effect is even stronger for vertical wells (Figure 5.19 to 5.22$)$.

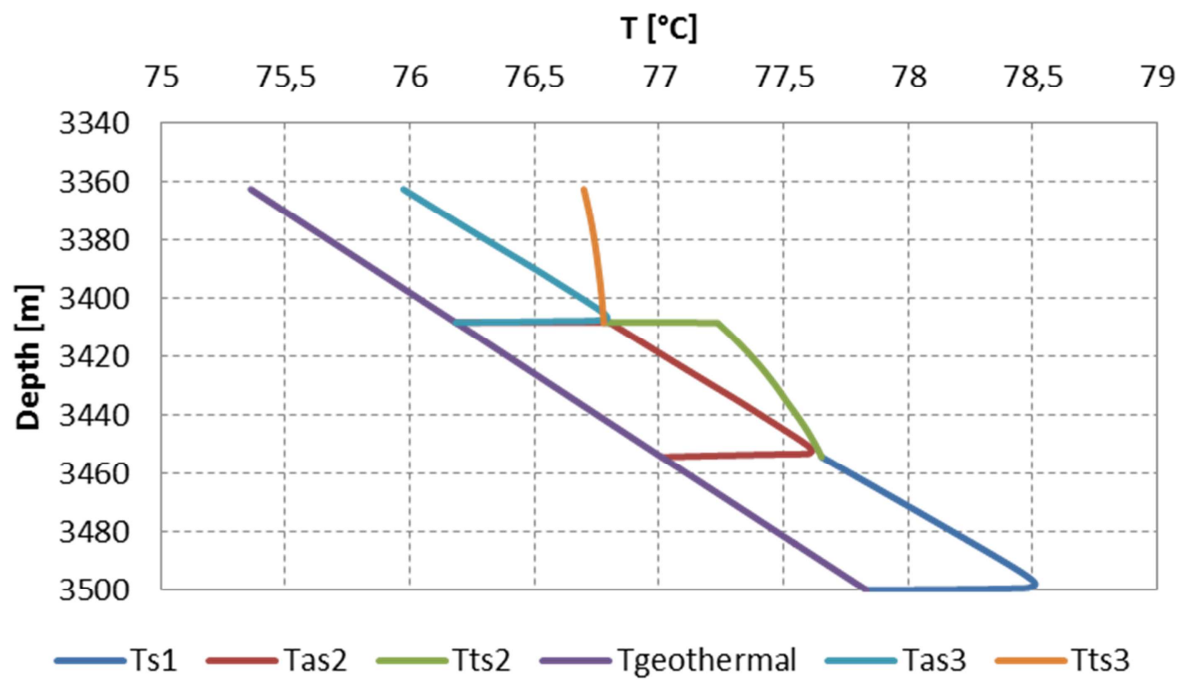

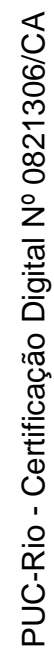

Figure 5.19 - Well temperature:T(z, t=0h).

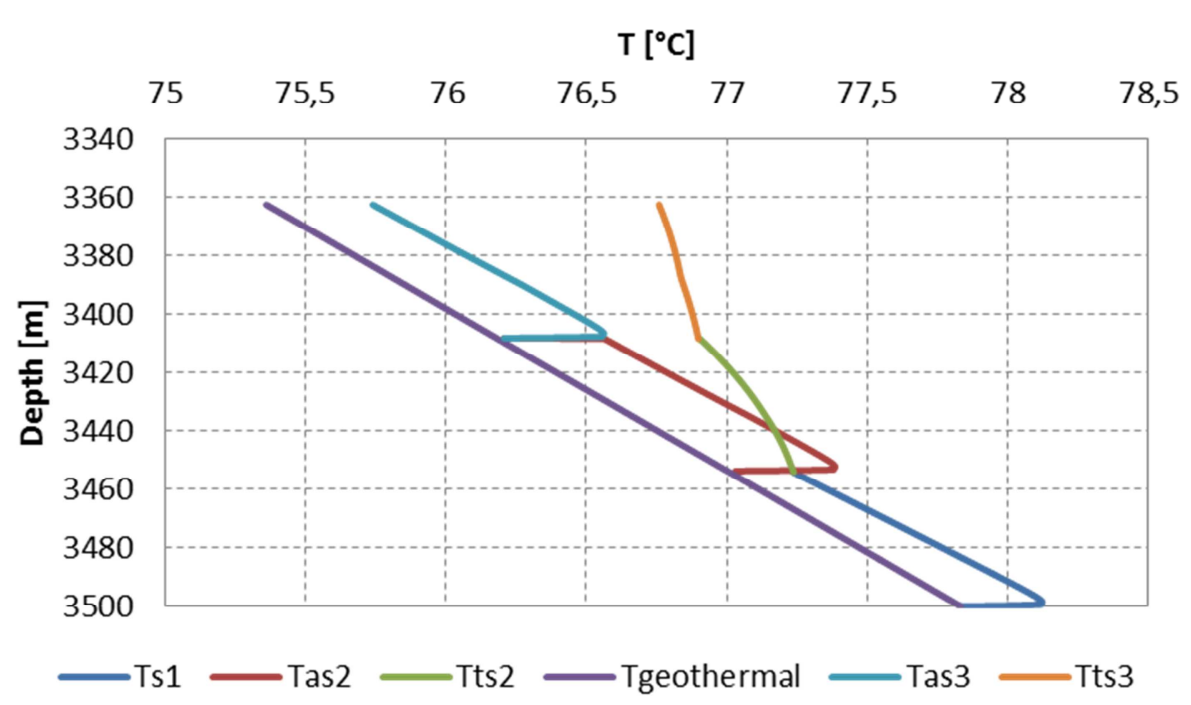

Figure 5.20 - Well temperature:T(z, t=3h). 


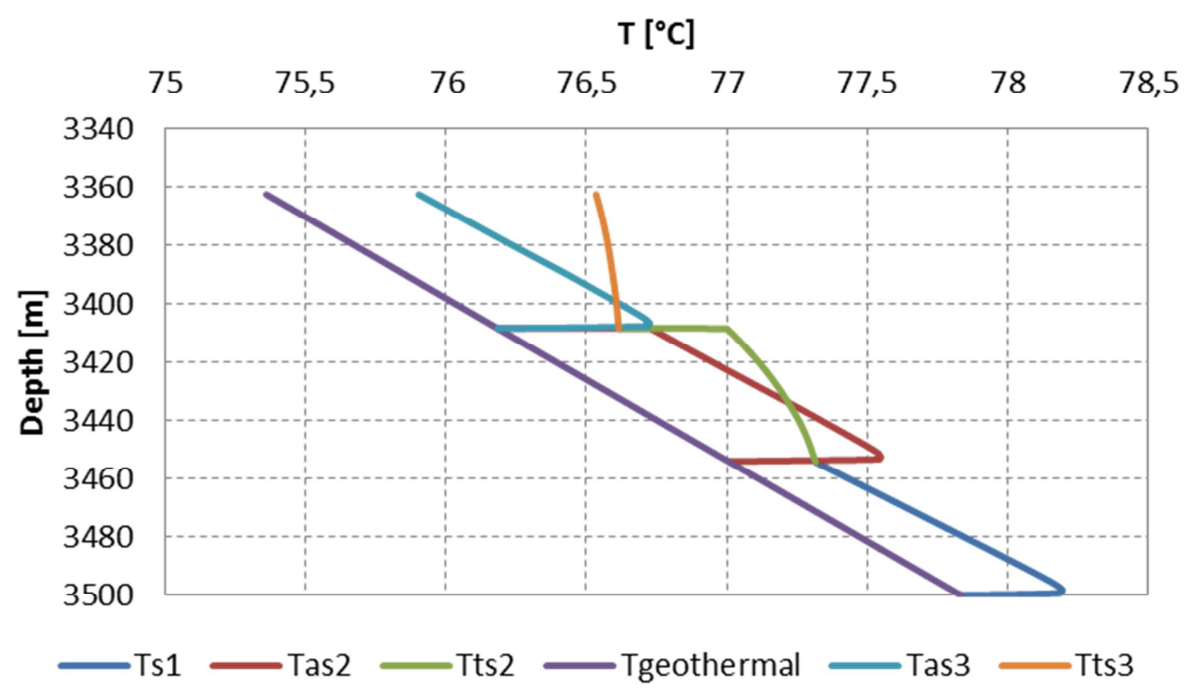

Figure 5.21 - Well temperature: $\mathrm{T}(\mathrm{z}, \mathrm{t}=24 \mathrm{~h})$.

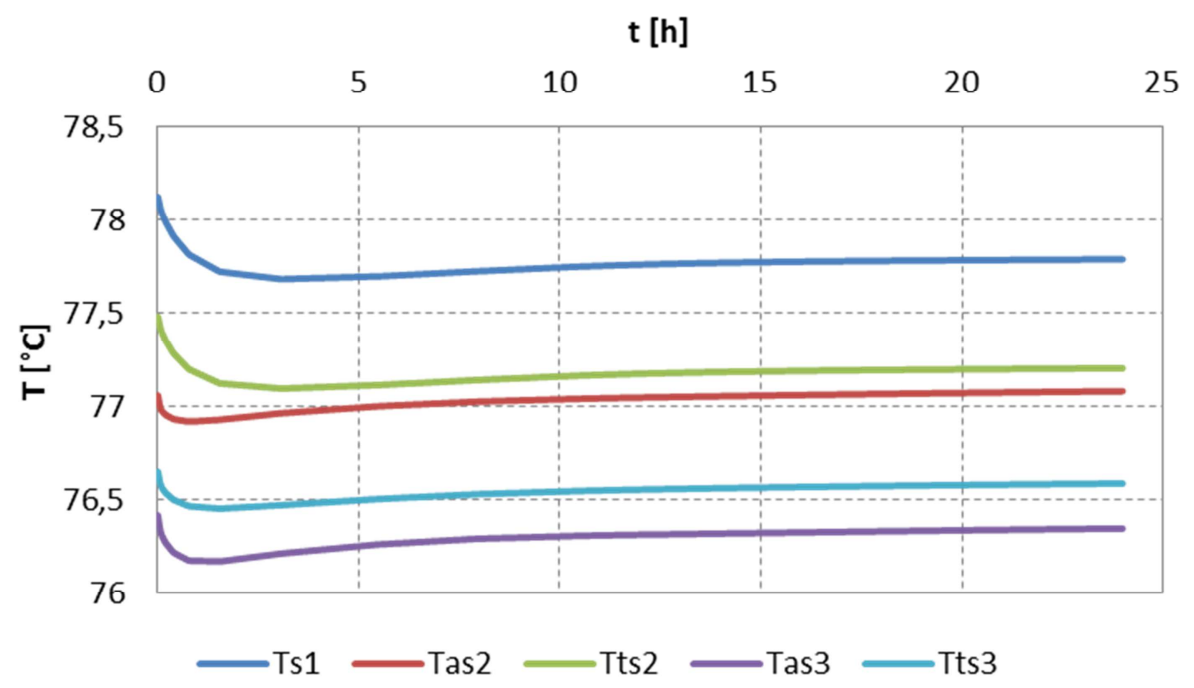

Figure 5.22 - Well temperature: $\mathrm{T}(\mathrm{z}=$ half segment, $\mathrm{t})$. 


\begin{tabular}{|c|c|c|c|c|}
\hline Oil data & Water data & Well data & Formation data & Other data \\
\hline$C_{p o}=0.64\left[\frac{B T U}{l b^{\circ} \mathrm{F}}\right]$ & $C_{p w}=0.95\left[\frac{B T U}{l b^{\circ} F}\right]$ & $R_{w b}=6[i n]$ & $K_{h}=50[\mathrm{mD}]$ & $K_{c e}=1\left[\frac{B T U}{h f t^{\circ} F}\right]$ \\
\hline$K_{o}=0.08\left[\frac{B T U}{h f t^{\circ} F}\right]$ & $K_{w}=0.34\left[\frac{B T U}{h f t^{\circ} F}\right]$ & $R_{c o}=4.81[i n]$ & $K_{v}=5[m D]$ & $K_{c}=K_{t}=10\left[\frac{B T U}{h f t^{\circ} F}\right]$ \\
\hline$\mu_{o}=0.6[c P]$ & $\mu_{w}=0.34[c P]$ & $R_{c i}=4.27[i n]$ & $\phi=0.2$ & $e=0.045 e-3[\mathrm{~m}]$ \\
\hline$\rho_{o}=36\left[\frac{l b}{f t^{3}}\right]$ & $\rho_{w}=64\left[\frac{l b}{f t^{3}}\right]$ & $R_{t o}=2.75[i n]$ & $\begin{array}{l}c_{t}=c_{f}+S_{o} c_{0}+S_{w} c_{w}= \\
=2.2 e-5\left[\frac{1}{p s i}\right]\end{array}$ & $q=13.248\left[\frac{m^{3}}{h}\right]$ \\
\hline$\beta_{o}=0.0009\left[\frac{f t^{3}}{f t^{3 \circ} F}\right]$ & $\beta_{w}=0.0001\left[\frac{f t^{3}}{f t^{3}{ }^{\circ} F}\right]$ & $R_{t i}=2.41[i n]$ & $D_{\text {ref }}=11480[\mathrm{ft}]$ & $g=9.80665\left[\mathrm{~m} / \mathrm{s}^{2}\right.$ \\
\hline$c_{0}=0.9 e-5\left[\frac{1}{p s i}\right]$ & $c_{w}=0.1 e-5\left[\frac{1}{p s i}\right]$ & $\gamma=0.9$ & $P_{\text {ref }} @ D_{\text {ref }}=5920[p s i]$ & $C_{d}=0.5$ \\
\hline$S_{0}=0.75$ & $S_{w}=0.25$ & & $T_{\text {ref }}=136 @ D_{\text {ref }}\left[{ }^{\circ} \mathrm{F}\right]$ & $A_{v}=6.27\left[\mathrm{in}^{2}\right]$ \\
\hline \multirow[t]{6}{*}{$\begin{array}{l}\lambda_{o}=\frac{K_{r o}}{\mu_{o}}=\frac{S_{0}^{2}}{\mu_{o}} \\
=0.94\left[\frac{1}{c P}\right]\end{array}$} & $\begin{array}{l}\lambda_{w}=\frac{K_{r w}}{\mu_{w}}=\frac{S_{w}{ }^{2}}{\mu_{w}} \\
=0.18\left[\frac{1}{c P}\right]\end{array}$ & & $C_{p f}=0.3\left[\frac{B T U}{l b^{\circ} F}\right]$ & $\begin{array}{l}\text { Choke profile: } 100,12,9,6,3, \\
2,1,0 \text { (\% Open) }\end{array}$ \\
\hline & & & $K_{f}=1.75\left[\frac{B T U}{h f t^{\circ} F}\right]$ & $\lambda_{t}=\lambda_{o}+\lambda_{w}=1.12\left[\frac{1}{c P}\right]$ \\
\hline & & & $\rho_{f}=135\left[\frac{l b}{f t^{3}}\right]$ & $\rho_{t}=\rho_{o} \frac{\lambda_{o}}{\lambda_{t}}+\rho_{w} \frac{\lambda_{w}}{\lambda_{t}}=40.5\left[\frac{l b}{f t^{3}}\right]$ \\
\hline & & & $h_{f}=300[f t]$ & \\
\hline & & & $g_{t}=0.0167\left[\frac{0}{f t}\right]$ & \\
\hline & & & $c_{f}=1.5 e-5\left[\frac{1}{p s i}\right]$ & \\
\hline
\end{tabular}

Table 5.1 - Data used in simulation of the cases 1 and 2. 


\section{6 \\ Data Analysis}

A decade after the first IW had been installed, an integrated modeling and optimization framework, shown in Figure 6.1, was proposed by Brouwer (Brouwer, Nædal et al. 2004). This paper describes how established concepts from control engineering and meteorology can be used for production and reservoir management. Since then, research and development were initiated in areas of reduced order modeling, optimization, monitoring systems, data analysis and assimilation.

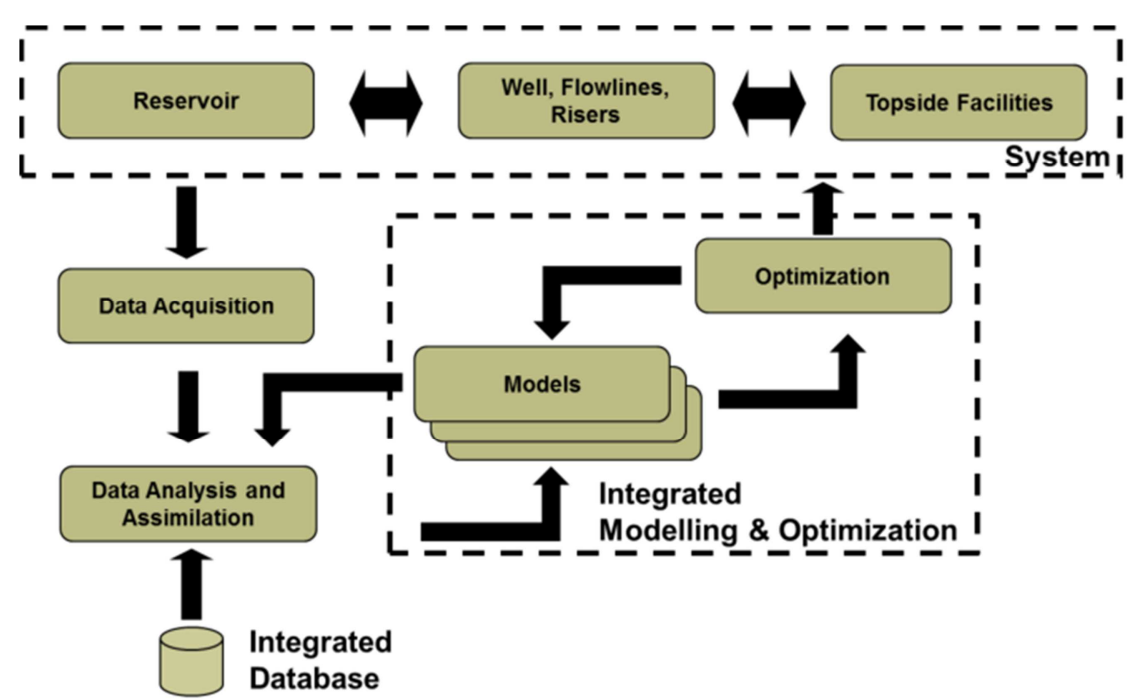

Figure 6.1 - Integrated modeling and optimization framework.

Data analysis can be defined as the procedure used to transform data into knowledge along the value chain path for making decisions as shown in the pyramid chart of the Figure 6.2. Data analysis and assimilation aims to provide the necessary information for model calibration and uncertainty minimization to avoid unreliable decision making. Typically software tools commercially available support all data analysis procedures: data acquisition, pre-processing, post-processing and interpretation without integrated modeling and optimization in mind. 


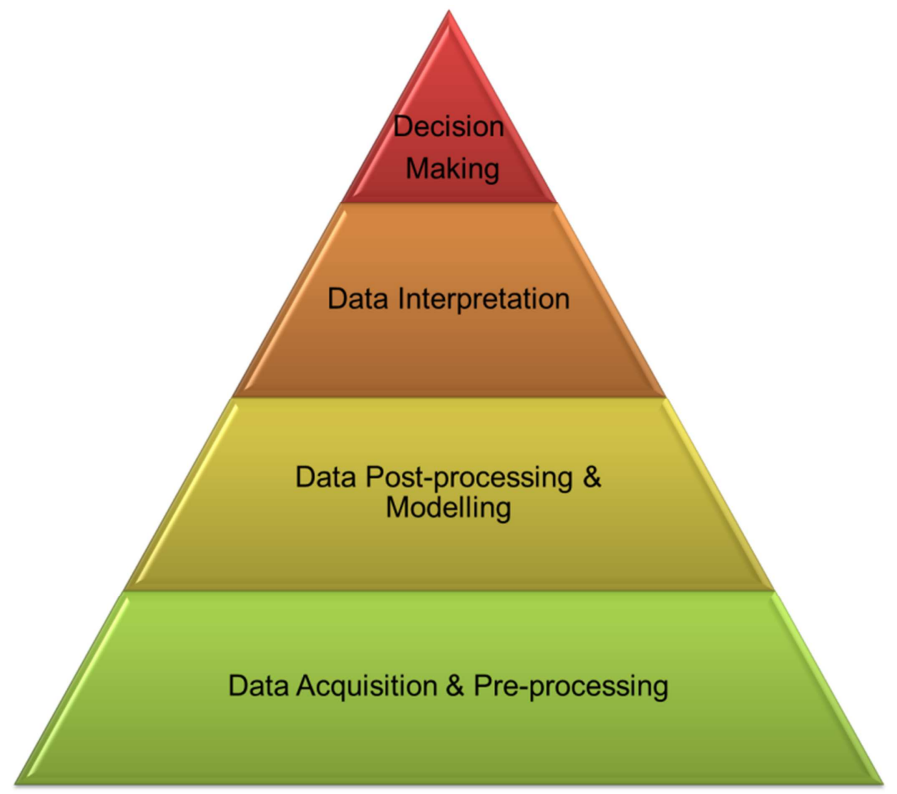

Figure 6.2 - Value chain pyramid chart.

Data acquisition and pre-processing deals with issues such as: sampling, denoising, outlier removal, compression and time synchronization. As mentioned earlier, pre-processing is done by application, e.g. gas lift optimization and IW have its own application tools. The integrated modeling and optimization framework aims to use appropriate pre-processing to improve the quality of the data analysis and assimilation procedure. One way of doing so is to perform in one place the pre-processing of the real-time data that is used by the different applications. This implies that the data from the production system are viewed as a single data set. Denoising, outlier removal and missing data can then be treated in a traceable manner, assuring the same level of quality control to all measurements. Further, time synchronization of the measured values will be more robust. This can be used to improve predictions of mutual influences of processes with different time scales rather than today's practice of only using it to adjust the correct sample rate for each process (decimation) which is essential for field scale analysis (Aggrey, Davies et al. 2007). As can be seen in Figure 6.3 the centralized pre-processing can potentially improve the quality of the post-processing predictions. Although this approach has not yet been fully explored, a successful application of it can be found in offshore operations and maintenance (Friedemann, Varma et al. 2008). 


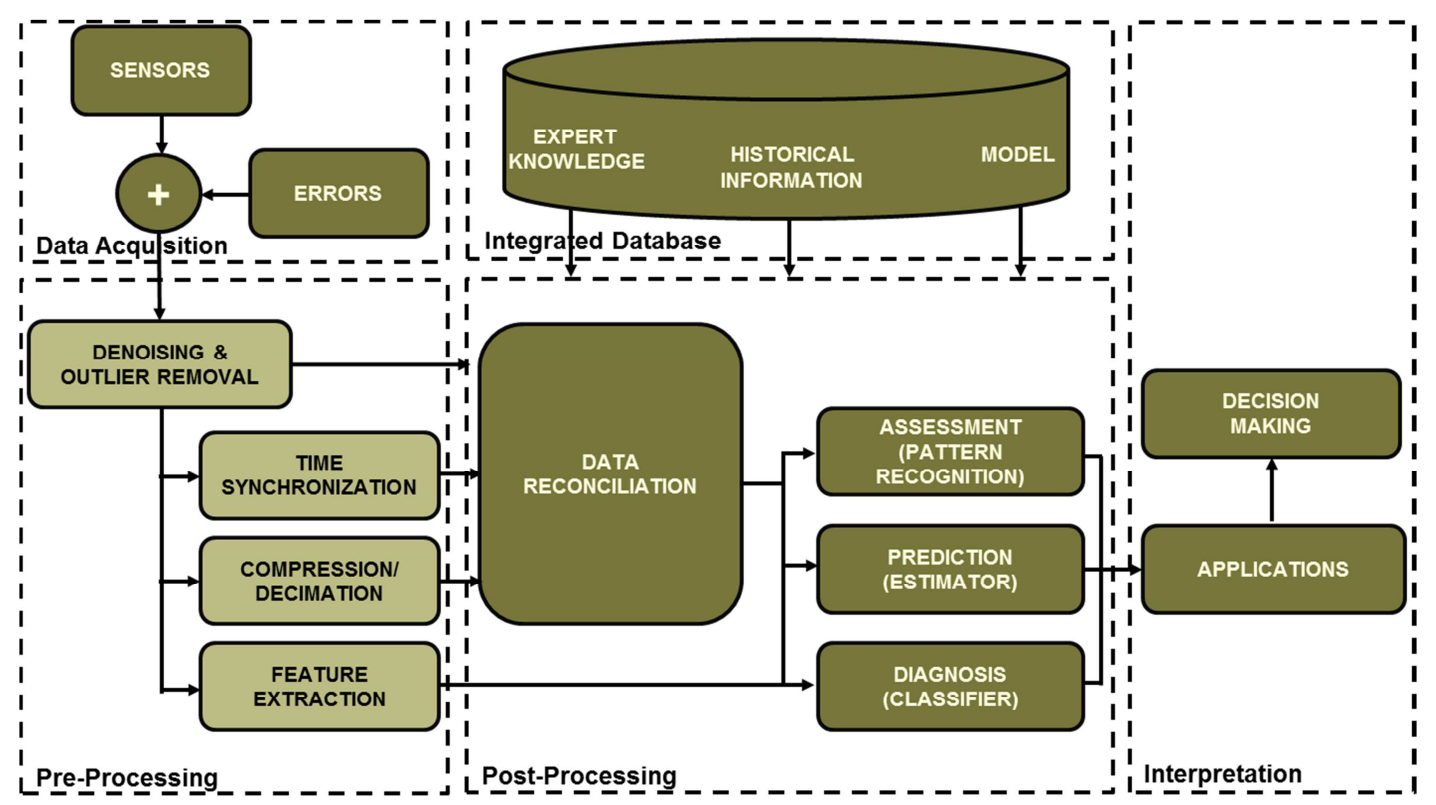

Figure 6.3 - Data analysis framework with centralized pre-processing.

A comprehensively architecture that relates the activities from the subsurface sensor to the engineer's office is illustrated in Figure 6.4. Bandwidth, noise, outliers, missing data, time synchronization, limited storage capacity, and data quality control are examples of common issues which need to be properly managed to avoid getting incorrect results during data analysis; not to mention issues such as sensor placement (important in transient analysis) and mechanical coupling (important in downhole seismic and acoustic sensors). Furthermore, downhole sensors cannot be calibrated without a well intervention. Nothing can be done when their measurement error changes. Only when the errors are large enough to be found using physical constraints it is possible to discard the incorrect measurements adequately. Published literature usually contains little general information on installation details or data acquisition issues, unless they refer to a specific application. 


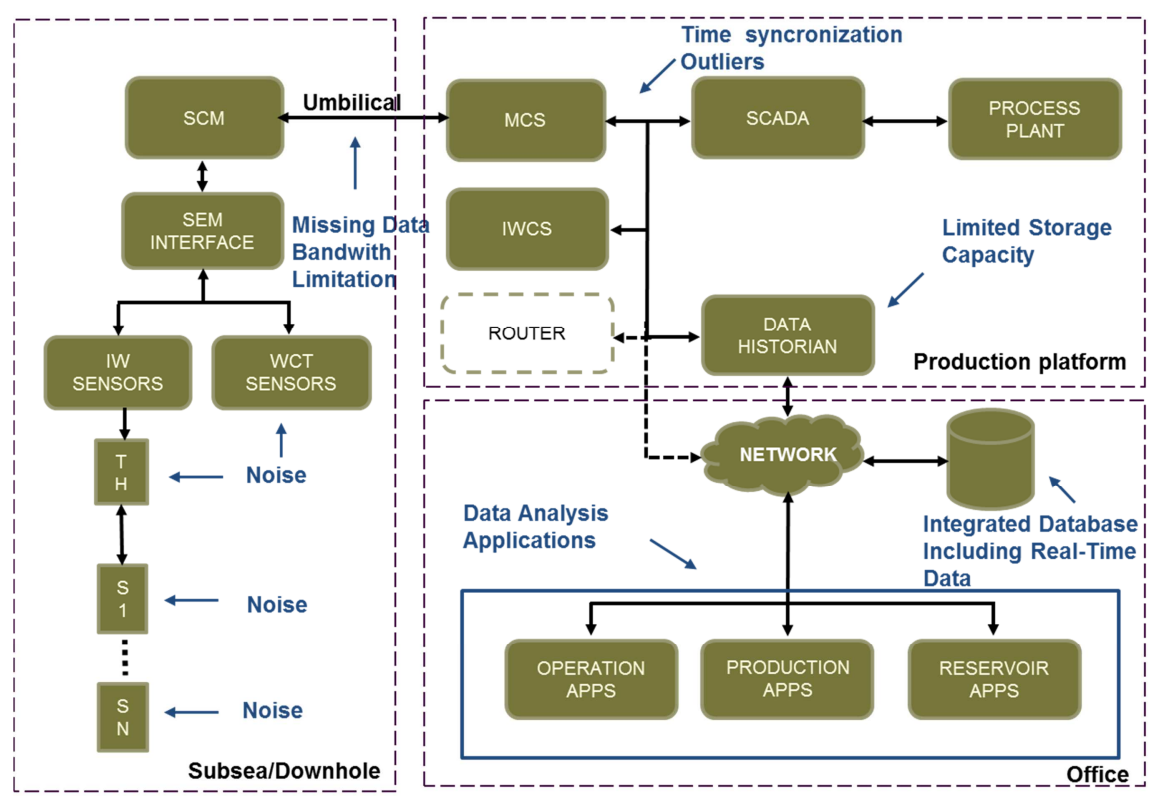

Figure 6.4 - Integrated IW architecture.

Applications employed for post-processing and data interpretation, data reconciliation and assimilation include reservoir management, advanced completions, well performance, flow assurance, well stimulation and condition monitoring. There are a variety of robust, commercial and in-house tools available to the operator for those applications individually. The challenge is to compare and integrate the results from these tools in a way that a reliable decision can be made. The integrated approach calls for the operator's chosen models to be fully integrated (from reservoir, wells/flowlines/risers to topside facilities). There is no off-the-shelf solution which efficiently mimics all the physical systems described above. Neither is there a set of applications which honors the needs of the different disciplines without important simplifications that result in losing the incremental value of the integrated framework (Hudson, Alves et al. 2011).

IW monitoring system has benefited in recent years from the measurement of new physical quantities combined with new data analysis methodologies. Instrumentation and other hardware advances have allowed more flexibility at the design and installation stage which have been enabling a variety of new applications. A basic IW monitoring system design framework is presented in Chapter 3 aiming to choose a fit for purpose IW monitoring system based on the available interpretation tools. The challenge for the integrated modeling and optimization framework is how to capture appropriately the mutual influence among processes in order to improve the decision making. 
Due to the integrated production and optimization framework real-time data is growing towards unmanageable amounts. Current available technologies are able to collect and transmit data from downhole, subsea and surface production units to wherever they are needed. Even though real deployments are still facing limitations as bandwidth, time synchronization, noise, missing data as well as standard criteria to data quality control and accessibility (Mathis and Thonhouser 2007). Some of those limitations are treated individually in the data analysis preprocessing at the application level and others not at all making traceability and effectiveness of the pre-processing in this scenario doubtful.

A step forward for data analysis at least for IW applications is centralized pre-processing as can be seen in Figure 6.5. It is not the intention to replace the existing post-processing and interpretation tools available but to improve them through a better framework. It is worth mentioning that criteria for data standardization and feature extraction are enablers for the success of this new framework.

Data analysis pre-processing as proposed in this thesis performs basically four procedures:

- Denoising/missing data interpolation/outlier removal;

- Compression/decimation;

- Time synchronization;

- Feature extraction.

They are used due to necessity of reliable data, free of outliers, missing data and measurement noise to improve the accuracy and effectiveness of the postprocessing and interpretation procedures. The additional procedures included refer to the mentioned issues, especially in subsea applications, as bandwidth limitation, time synchronization, data overload, and spatially distributed sensing. Feature extraction was also included to capture influences among processes.

Denoising/missing data interpolation/outlier removal procedure is studied by using RPCA (Robust Principal Component Analysis) via PCP (Principal Component Pursuit) to approximately restore and separate the signal from noise and outliers which is assumed sparse (Lin, Chen et al. 2010). Missing data are allowed and the procedure is done simultaneously through a surrogate convex optimization approach. 
Compression and time synchronization have been studied recently from Olsen (Olsen et al, 2011) and Aggrey (Aggrey, Davies et al. 2006) for transient and steady state signals. They are treated as future work in this thesis.

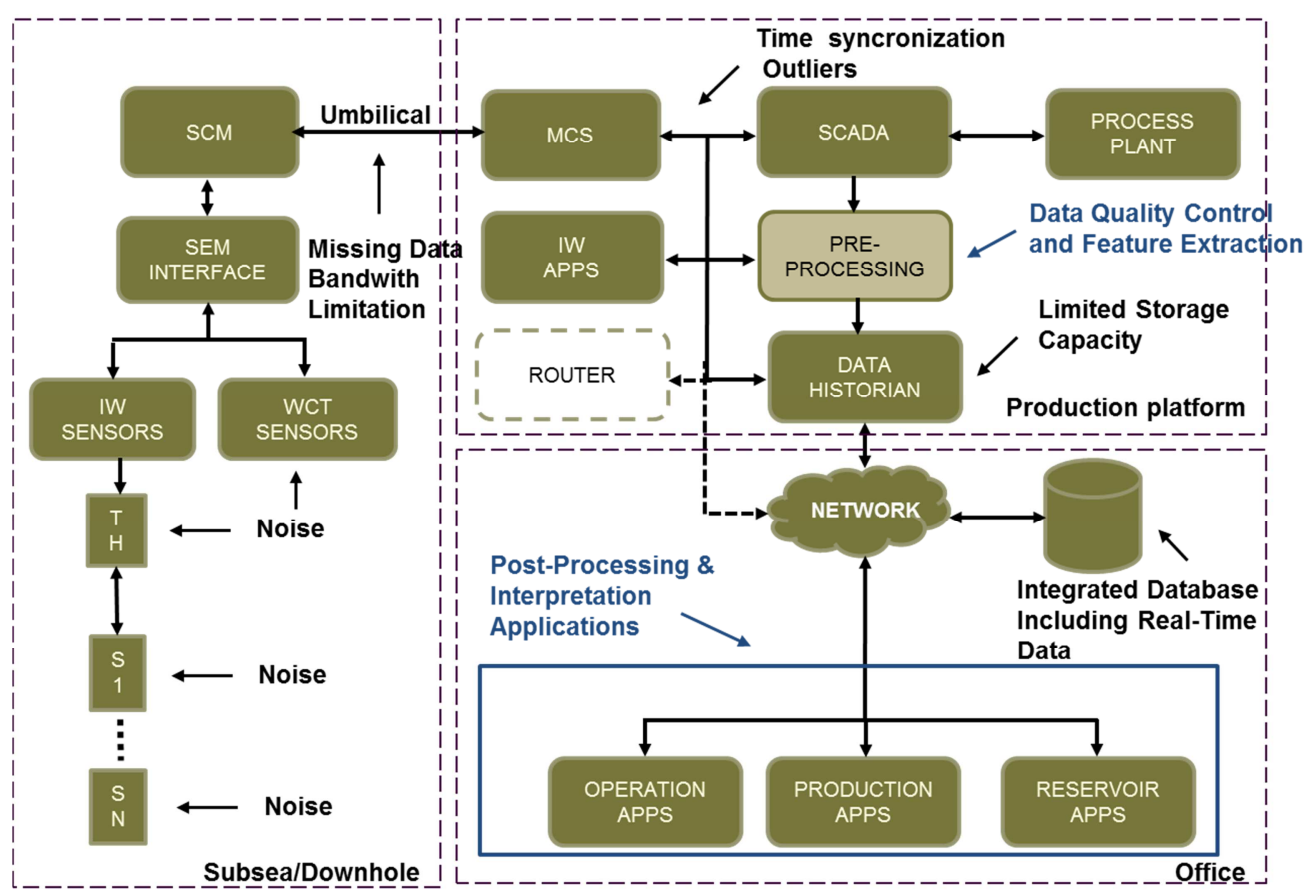

Figure 6.5 - Integrated IW application with centralized pre-processing.

Feature extraction is introduced by using DM (Coifman and Lafon 2006) which allows identification, e. g., of an abnormal behavior and can be used to improve pattern recognition, classification and estimation procedures of the data analysis post-processing and interpretation. In Chapter 7 and 8 we describe the pre-processing theory, validating and applying the new framework through the use of the non-isothermal dynamic well-reservoir model to generate synthetic "clean" data. Artificial noise, multiple outliers and missing data are introduced to corrupt the original "clean" data and then used as input to the pre-processing framework. The main goal is to automatically identify pressure and temperature transients. The cleansing and feature extraction procedures performances are compared against a state of the art one (Olsen 2011). The main contribution of this new framework is not only completing all cleansing tasks simultaneously with less sensitivity to outliers and missing data but also making possible the use multiple signals, including different ones, surveying the same phenomena. 


\section{7}

\section{Pre-processing Theory Background}

Real monitoring systems suffer of various types of data corruption. They might be caused by installation, telemetry, sensors and aging, being classified as: random, systematic, and gross errors. Specifically for downhole monitoring we emphasize connection, coupling, and placement induced errors as the ones that nothing can be done after installation without a costly intervention.

In this chapter, first we describe random, systematic, and gross errors, with some real examples, and afterwards how to model them. Second, we present common techniques used in downhole monitoring system to treat noise and outliers introducing RPCA via PCP (Candès, Li et al. 2011) which is, roughly speaking, a signal separation problem, as one of the pieces of our framework. Finally, we present a feature extraction technique based on DM (Coifman and Lafon 2006) and our full pre-processing framework.

\section{1}

\section{Corrupted Measurement Data}

Random, systematic and gross errors are the major types of errors in measurement systems. As the name states the random errors are characterized by unknown and unpredictable changes. They are assumed to be additive and modeled using the equation 7.1:

$$
y_{t, n o i s e}=y_{t}+r
$$

Where:

$$
r \square N\left(0, \sigma^{2}\right)
$$

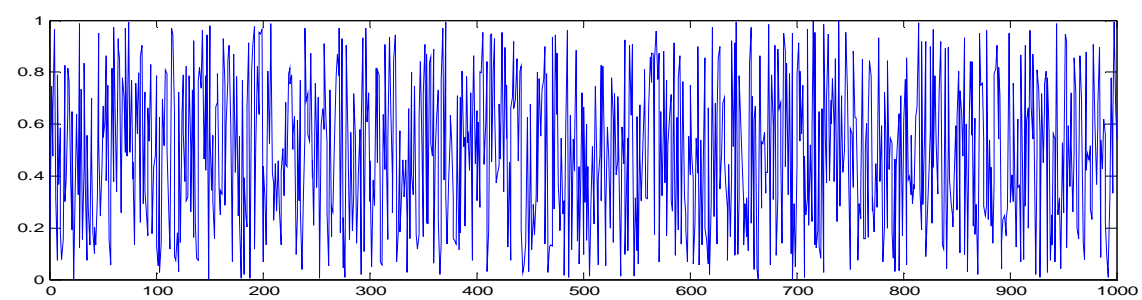

Figure 7.1 - Random noise. 
The systematic errors occur mainly due to a flaw in the equipment. Normally they can be minimized or even eliminated knowing the equipment aging and environment impact (signal trend model). They can be arranged in three minor groups classified as: bias, drift and degradation (Figure 7.2). Bias consists basically in a constant variation over time, drift is deviations occurring after sensor calibration, and degradation is basically the amount of noise getting bigger due to the aging of the monitoring system. All those are affected by an incorrect equipment specification or an inappropriate environment. The latter is common in downhole monitoring systems due to the uncertainty associated to the environment.
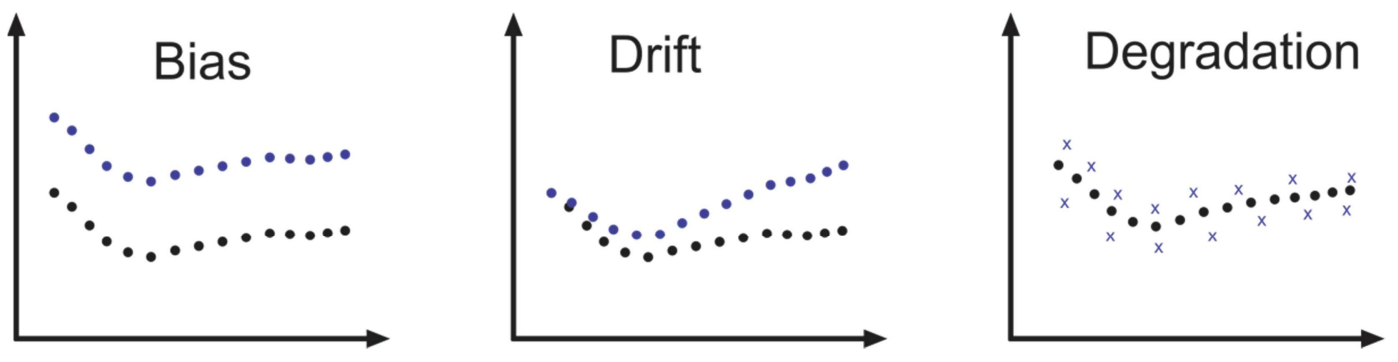

Figure 7.2 - Systematic errors.

The descriptions above allow us to illustrate a real signal behavior which is normally a mixture of them. An example of degradation and drift can be seen in Figure 7.3. The signal was gathered from a PDG installed at $2905.42 \mathrm{~m}$ (TVD True Vertical Depth) in a water injector horizontal well. The "clean" signal, without the effect of drift and degradation, is the green one and as the sensor is getting old degradation and drift is becoming larger. It is important to observe that these types of errors are normally identified by chance or when the measurement is clearly unfeasible. This example was made measuring noise and drift right after installation and right after retrieval. 


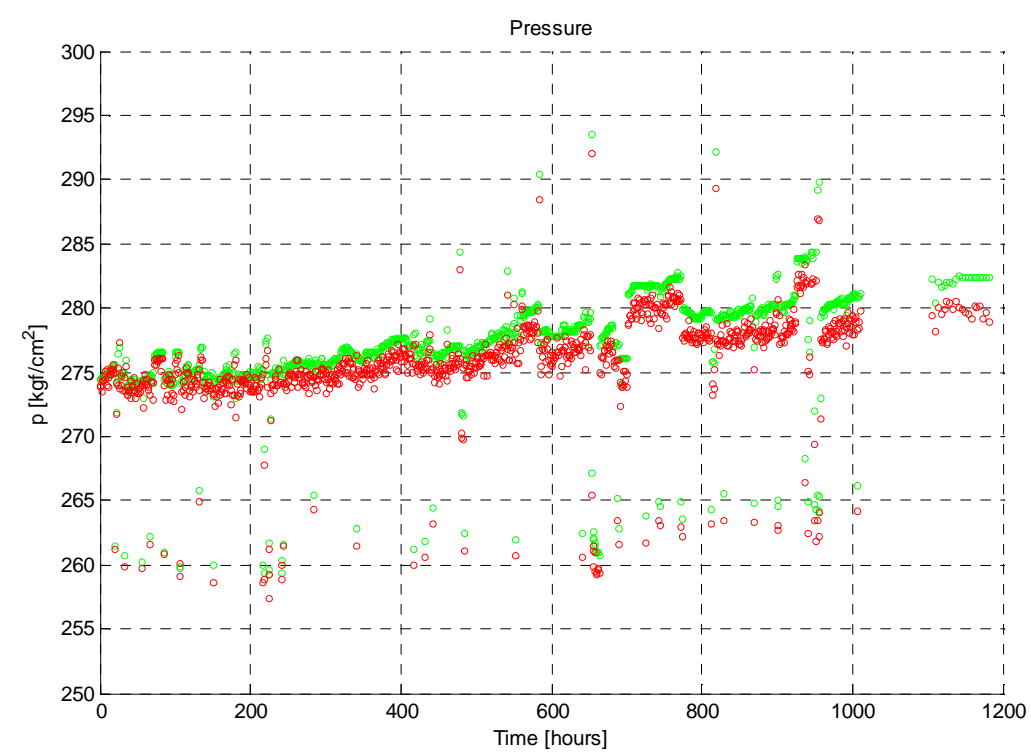

Figure 7.3 - Noise and drift of a real PDG (the green signal is the "clean" one).

An example of drift due to reading unit noise in a distributed temperature sensing is presented using a published noise model on anti-stokes/stokes measurements as function of signal intensity (Hadley and Kimish 2008).

The measured temperature in a distributed temperature sensing system is calculated by the equation 7.2 as function of reference measurement, differential loss and anti-stokes/stokes ratio:

$$
\frac{1}{T}=\frac{1}{T_{0}}-\frac{1}{D L} \ln \left(\frac{I}{I_{0}}\right)
$$

Where:

$D L$ - Differential Loss

$I$ - anti-stokes and stokes intensity ratio

$I_{0}-$ anti-stokes and stokes intensity ratio at reference temperature $\left(T_{0}\right)$

Let's assume that reference temperature and differential loss have a negligible impact on the temperature uncertainty. From the reference paper, the noise of stokes and anti-stokes signals are modeled by the equation 7.3:

$$
\sigma=\sqrt{c_{1}^{2}+I_{\text {signal }} c_{2}^{2}}
$$

Where:

$c_{1}, c_{2}-$ constants;

$I_{\text {signal }}$ - signal intensity. 
Applying equation 7.3 to calculate the uncertainty of the temperature gives as result the equation 7.4 assuming independent and identically distributed measurements distributions:

$$
\sigma^{2}=\left(\frac{1}{\bar{I}_{s}}\right)^{2} \sigma_{A S}^{2}+\left(\frac{\bar{I}_{A S}}{\bar{I}_{S}^{2}}\right)^{2} \sigma_{S}^{2}
$$

The equation 7.4 shows that the temperature uncertainty varies with signal intensity which is function of the position. As interpretation methods used for distributed temperature sensing depend on temperature gradient they are directly impacted. Therefore this systematic error should be taken into account to minimize misinterpretations. It is worth mentioning that signal processing as trace averaging and moving averaging within trace normally used to minimize noise is not able to remove this kind of effect and it should be removed before any signal processing be done. An example of this effect in a real distributed temperature measurement can be seen in Figure 7.4. The temperature signal was gathered from a DTS installed in a producer horizontal well (onshore) where we are interested in the $505 \mathrm{~m}$ of the horizontal length starting at $854 \mathrm{~m}$ (MD). The lines black and blue corresponds to the temperature signals after signal processing with and without systematic error correction due to noise dependence with position.

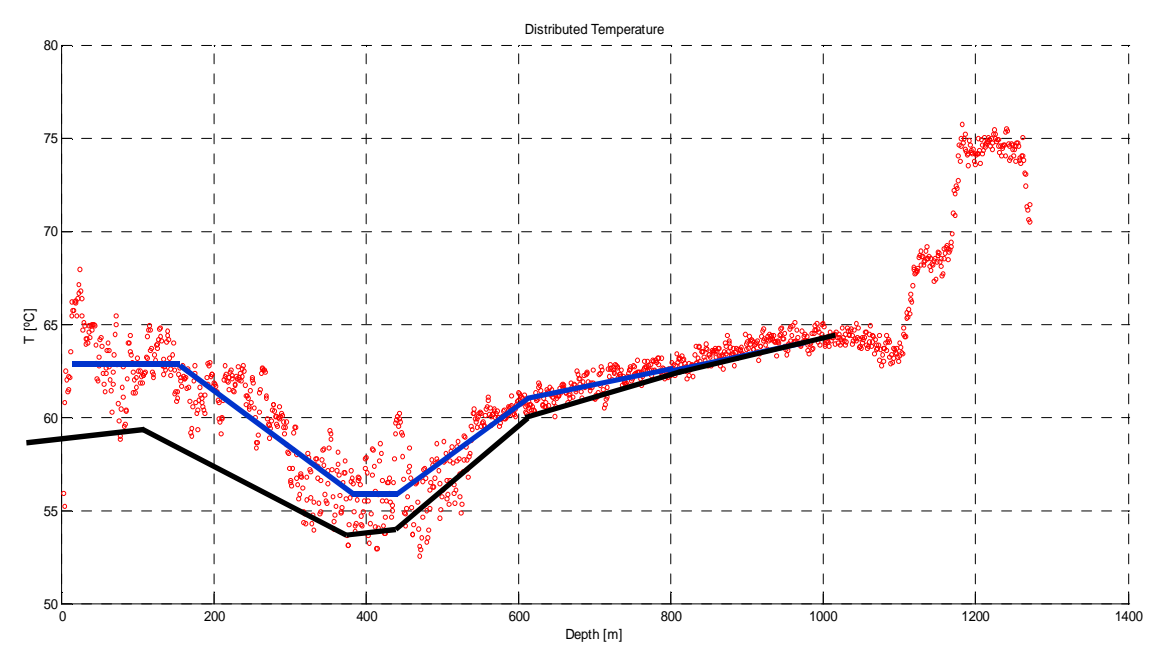

Figure 7.4 - Temperature gradient profile error due to reading unit noise model in a real temperature measurement (blue line without correction).

The last major type of error is the gross errors. They occur when the instrument goes on complete failure and they are understood as errors associated 
to the production process. Typically they are modeled as a product of a switching distribution times a Gaussian distribution but Heavy-Tailed Distributions or a linear combination of Gaussian Distributions may also be used:

$$
y_{t, \text { outlier }}=y_{t, \text { switching }} \cdot y_{t, \text { corrupting }}
$$

It should be noted that the outlier is assumed to be an additive error over the original signal and the switching distribution is typically Bernoulli or Markov.

\section{2}

\section{Denoising and Outlier Removal}

All major types of measurement errors described in the last section can be minimized through the use of filters. There are only 5 of them heavily used in downhole monitoring systems signals: mean filter, median filter, maximumminimum filter, moving average filter and wavelet filter. In addition to them we introduced a non-conventional RPCA via PCP as a new type of filter for simultaneous denoising and multiple outlier removal technique.

\subsection{1}

\section{Conventional Filters}

In this section we describe the most used filters in downhole monitoring systems especially for transient analysis applications. As mentioned earlier they consist of 5 filters:

The mean filter is the simplest one and consists in smoothing the signal by taking the average value of the last $w$ measurements. This filter can be modeled by the equation 7.6:

$$
y_{t, \text { mean }}=\frac{1}{w+1}\left(y_{t,-w}+\ldots+y_{t}\right)
$$

To illustrate the mean filter performance we have chosen a synthetic signal built using piece-wise regular polynomials as a reference clean signal. This signal is available in the Wavelab 850 MatLab $^{\mathrm{TM}}$ Toolbox from Stanford University. A random noise normally distributed with mean 0 and standard deviation 10 is added to the clean signal and filtered (with $w=30$ ) afterwards (Figure 7.5). The 
result is then compared with the clean signal through $L 2$ norm of the relative error giving $6.48 \times 10^{-2}$.

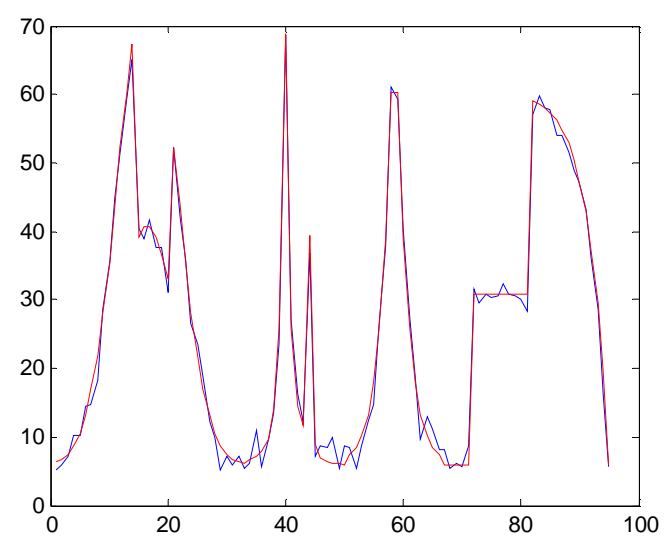

Figure 7.5 - Mean filter performance (red is the clean signal).

The median filter works in a similar way of the mean one but instead of taking the mean of the last $w$ values it takes the median of them. This filter can be modeled by the equation 7.7 :

$$
y_{t, \text { median }}=\left\{\begin{array}{l}
\left(y_{t,-w}<\ldots y_{t,-w / 2} \ldots<y_{t, 0}\right), \forall w-\text { even } \\
\left(y_{t,-w}<\ldots 1 / 2\left(y_{t,-(w+1) / 2}+y_{t,-(w-1) / 2}\right) \ldots<y_{t, 0}\right), \forall w-o d d
\end{array}\right.
$$

As before using the synthetic signal and noise, and with the same $w$, we evaluate the performance of the median filter making a comparison between the filtered and the clean signals. The result gives $1.84 \times 10^{-1}$ ( $L 2$ norm of the relative error).

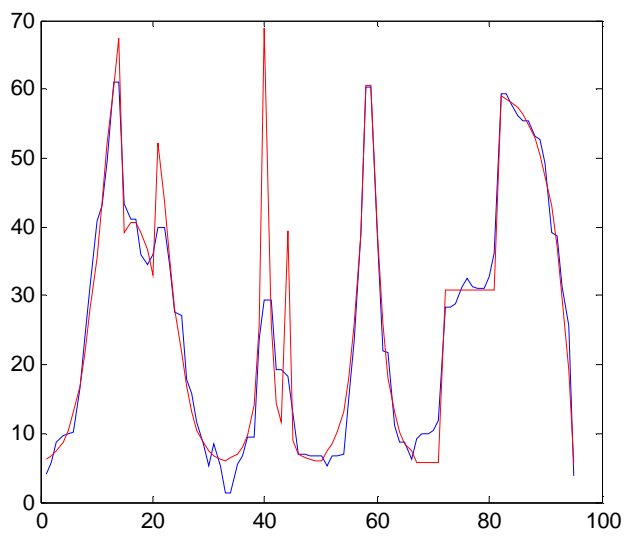

Figure 7.6 - Median filter performance (red is the clean signal). 
The maximum-minimum filter consists in taking a measurement and replacing it when the value is lower than a minimum or higher than a maximum value. This filter can be modeled by the equation 7.8 :

$$
y_{t, \text { max }-\min }=\left\{\begin{array}{ccc}
y_{t} & \forall & y_{t} \in\left[y_{t, \text { min }}, y_{t, \text { max }}\right] \\
y_{t, \text { min }} & \forall y_{t}<y_{t, \text { min }} \\
y_{t, \text { max }} & \forall y_{t}>y_{t, \text { max }}
\end{array}\right.
$$

The performance of this maximum-minimum filter doing the same comparison as before gives as result $6.96 \times 10^{-2}$ ( $L 2$ norm of the relative error).

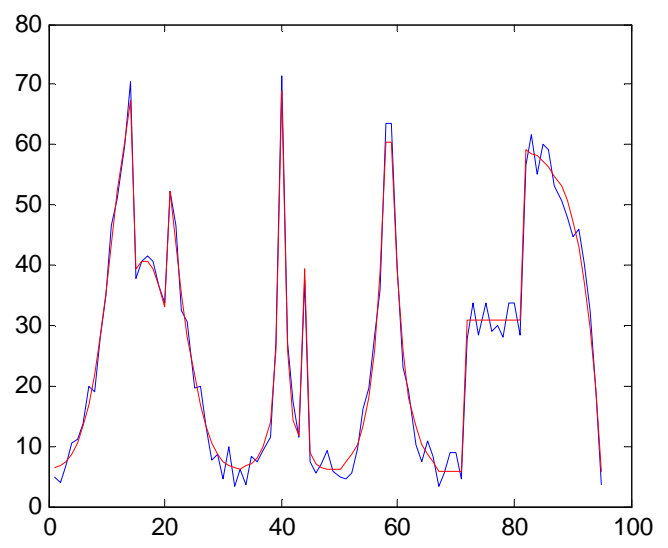

Figure 7.7 - Maximum-minimum filter performance (red is the clean signal).

The moving-average filter works taking a number of measurements at a specific time and producing an average value of the taken values. The number of measurement defines a window in time which can be asymmetric or symmetric regarding this specific time. It should be noted that for the asymmetric case it is expected phase problems. Depending on the signal this can be a problem. This filter can be modeled by the equation 7.9:

$$
y_{t, m v a}=\frac{1}{w+1}\left(y_{t-w}+\ldots+y_{t}\right)
$$




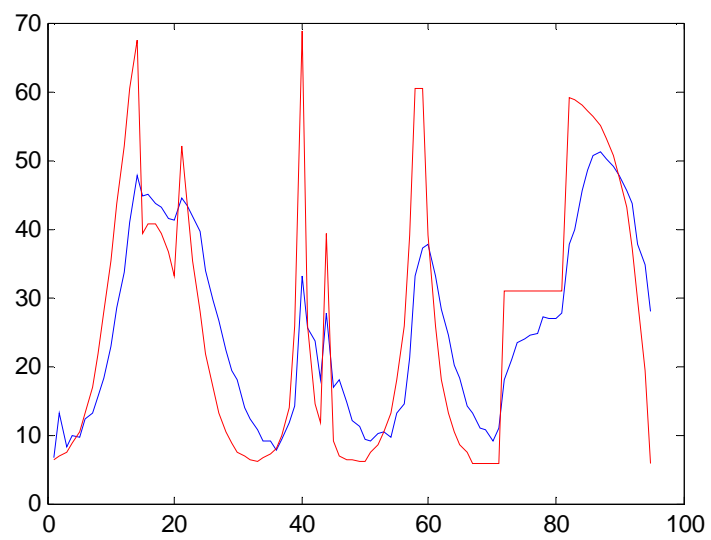

Figure 7.8 - Moving-average filter performance (red is the clean signal).

The performance of this moving-average filter doing the same comparison as before gives as result $3.13 \times 10^{-1}$ ( $L 2$ norm of the relative error).

The wavelet filter has proven to be valuable for denoising in particular the wavelet shrinkage method proposed by (Donoho and Johnstone 1994) which provides min-max optimal solution. Today many wavelets threshold techniques are available but we focus on those studied by (Olsen 2011) which is aligned with this thesis goals.

Waveshrink method is based on the principle that noise has smaller wavelet coefficients than the underlying signal. Therefore if we apply a threshold function to penalize those coefficients, while preserving the signal, we eliminate the noise. Basically it has three steps: wavelet transform the noisy signal, apply a shrinkage operator to the resulting coefficients, and apply the inverse of the wavelet transform to restore the signal. Mathematically it can be expressed as:

$$
\hat{y}_{t}=W^{-1}\left(D\left(W\left(y_{t, n o i s e}\right), \lambda\right)\right)
$$

Where:

$W$ - wavelet transform

$D$ - shrinkage operator

$\lambda$ - Threshold

$W^{1}-$ inverse wavelet transform

The main requirement of the Waveshrink method is an equally spaced signal and an appropriate primary resolution level. Depending on the application of the 
correct choice of the wavelet family and scaling function, the shrinkage operator which can be hard, soft, or a function (linear, Firm, Garrote, SCAD - Smoothly Clipped Absolute Deviation, etc.), the threshold estimator, and the noise estimator can be completely different. An extensive work by (Olsen 2011) indicated Spline39 wavelet, SCAD shrinkage rule, RED (Regression Estimated Decline) threshold estimator, and RMAD (Robust Median Absolute Deviation) noise estimator as recommended choices for pressure transient analysis.

The performance of the Waveshrink method is illustrated using the same signal and noise as before for the hard and soft shrinkage operator (Figure 7.9) which can be mathematically expressed as:

$$
\begin{aligned}
& D\left(W\left(y_{t, \text { noise }}\right), \lambda\right)=W\left(y_{t, \text { noise }}\right) \cdot \mathbf{1}_{\left|W\left(y_{t, \text { noise }}\right)\right|>\lambda} \\
& D\left(W\left(y_{t, \text { noise }}\right), \lambda\right)=\operatorname{sign}\left(W\left(y_{t, \text { noise }}\right)\right) \max \left(0,\left|W\left(y_{t, \text { noise }}\right)\right|-\lambda\right)
\end{aligned}
$$
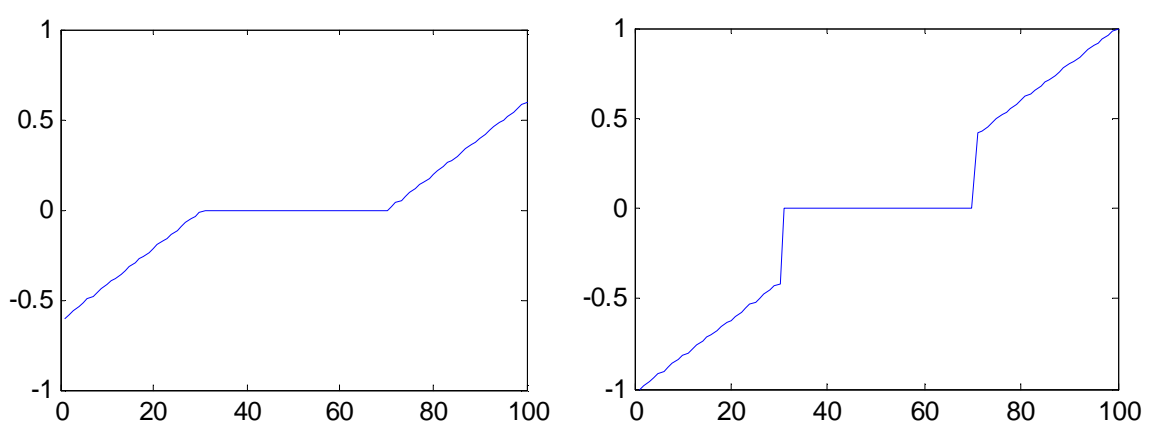

Figure 7.9 - Soft and hard shrinkage operator, respectively.

To accomplish the comparison we choose Symlet 4 (level 3) as the wavelet family and threshold of 3.16 . The results were $9.49 \times 10^{-2}$ and $9.01 \times 10^{-2}$ ( $L 2$ norm of the relative error) for the hard and soft threshold respectively as can be seen in Figure 7.10 and Figure 7.11. 


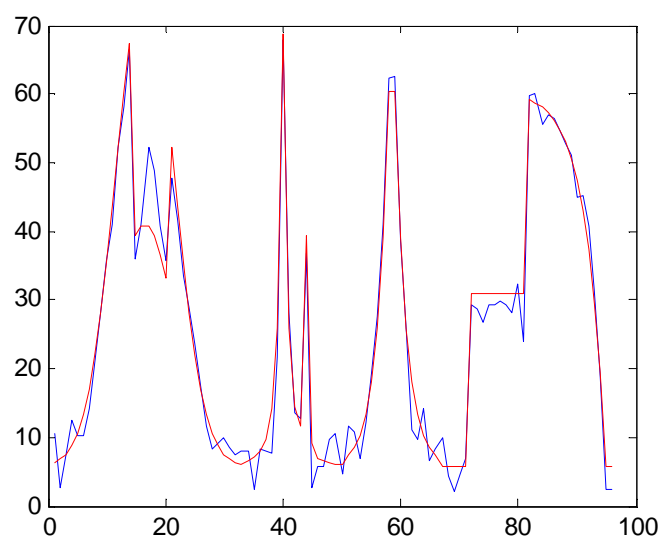

Figure 7.10 - Wavelet filter performance for the hard threshold (red is the clean signal).

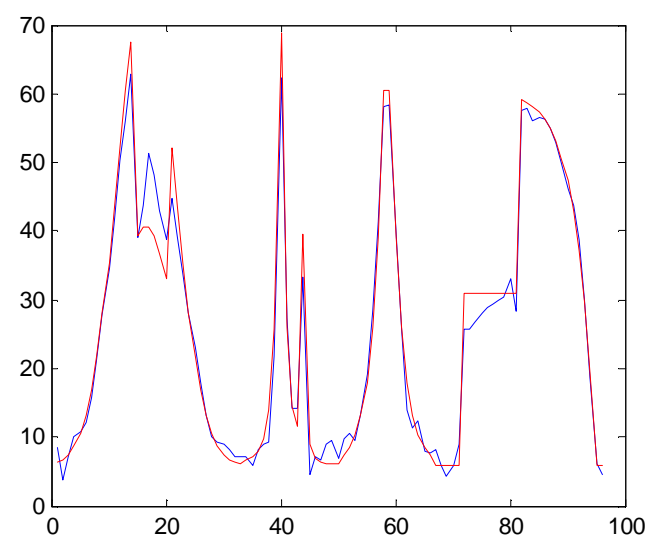

Figure 7.11 - Wavelet filter performance for the soft threshold (red is the clean signal).

\subsection{2}

\section{Robust PCA via Principal Component Pursuit}

In order to understand this robust PCA (Principal Component Analysis) algorithm, we firstly describe the PCA problem as a low rank minimization problem: given a matrix $D$, where columns are the observation vectors corrupted by Gaussian noise, find the optimal low-rank approximation in $l 2$-sense. Mathematically if we know $r$ we can formulate the problem as:

$$
\min _{L, N}\|D-L\|_{F}^{2} \quad \text { s.t. } \quad \operatorname{rank}(L) \leq r
$$

If we do not, as:

$$
\min _{L, N} \operatorname{rank}(A)+\frac{\alpha}{2}\|D-L\|_{F}^{2}
$$


Where

$D=L+N$;

$L$ - low rank signal;

$N$ - Gaussian noise.

The optimal solution for the former is given by: $L=U H(\Sigma) V^{T}$ where $H(\Sigma)=\Sigma \mathbf{1}_{|\Sigma|>\alpha}$ is the hard-threshold operator. In other words, the SVD (Singular Value Decomposition) of $L$ choosing the singular values higher than a positive $\alpha$. As the latter problem is NP (Non-Polynomial Time) hard normally the rank of $L$ is replaced by the sum of the singular values of $L$, i.e., its nuclear norm $\|L\|_{*}$ which leads to the following convex optimization problem (Recht, Fazel et al. 2010):

$$
\min _{L, N}\|L\|_{*}+\frac{\alpha}{2}\|D-L\|_{F}^{2}
$$

This new problem optimal solution is given by: $L=U S_{1 / \alpha}(\Sigma) V^{T}$ where $S_{1 / \alpha}(\Sigma)=\left\{\begin{array}{c}\Sigma-1 / \alpha, \Sigma>1 / \alpha \\ \Sigma+1 / \alpha, \Sigma<-1 / \alpha \\ 0\end{array} \quad\right.$ is the soft-threshold operator.

The well-known problem with the above methods is sensitivity to outliers (Figure 7.12). There are a lot of works on robustifying these kinds of methods, most of which consisting in first detecting and removing, and afterwards filling them through some heuristics.
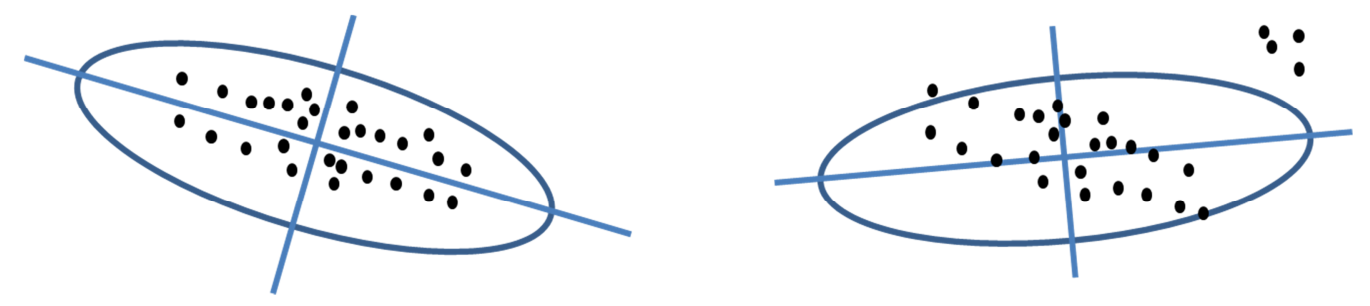

Figure 7.12 - Outliers problem.

A recent work by (Candès, Li et al. 2011) showed that if we assume lowrank signal and sparse outliers, an exact recovery of the signal is expected with high probability from arbitrary and completely unknown outliers corruption patterns if a convex optimization problem called PCP (Principal Component Pursuit) is solved. It is worth mentioning that this problem is different from the 
matrix completion problem introduced by (Candès and Recht 2009) which simultaneously detects the corrupted entries, and fits appropriately the low-rank component to the remaining reliable entries. Mathematically, an observation matrix $D \in \square^{m x n}$ may be decomposed as low-rank signal matrix $L$ and sparse outliers $O$ solving the following optimization problem:

$$
\min _{L, N}\|L\|_{*}+\lambda\|O\|_{1} \text { s.t. } D=L+O
$$

Where

$$
\begin{aligned}
& \lambda=\frac{1}{\sqrt{\max (m, n)}} \\
& L-\text { low rank signal; } \\
& O \text { - sparse outliers. }
\end{aligned}
$$

The exact recovery is achieved if the following bounds are provided for $L$ and $\mathrm{O}$ (Candès, Li et al. 2011):

$$
\operatorname{rank}(L) \leq \frac{\rho_{r} \min (m, n)}{\mu(\log (\max (m, n)))^{2}},\|O\|_{0} \leq \rho_{o} m n
$$

Where

$$
\begin{aligned}
& \mu \text { - coherence of D }(\mu \geq 1) ; \\
& \rho_{o}, \rho_{r}-\text { positive constants. }
\end{aligned}
$$

Notice that $\lambda=\frac{1}{\sqrt{\max (m, n)}}$ is not a tuning parameter which means that under the assumptions works with high probability for recovering any low-rank, incoherent matrix (Candès, Li et al. 2011).

Before we go further we present the relaxed version of the problem 7.13 (Zhou, Li et al. 2010) which includes noise and can also deal with missing data:

$$
\min _{L, O}\|L\|_{*}+\lambda\|O\|_{1} \text { s.t. }\|D-(L+O)\|_{F} \leq \delta
$$


The relaxed version of the problem can be seen as a denoising technique. Given a data matrix, it separates the signal and the noise in two other matrices: one low rank (signal) and one sparse (outiliers/noise) as presented in Figure 7.13.
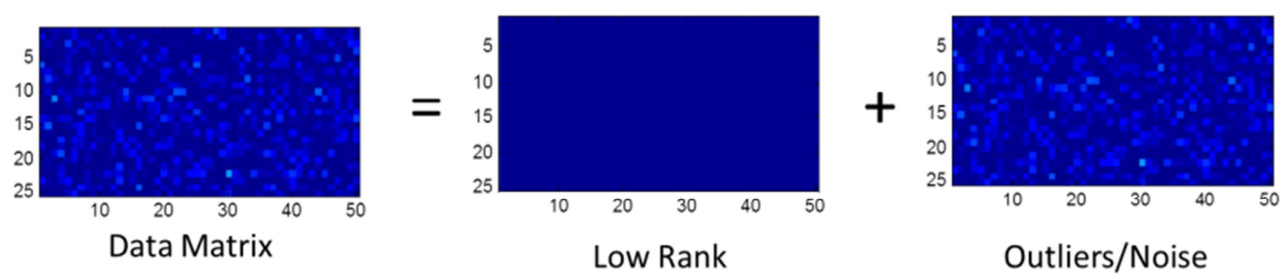

Figure 7.13 - PCP as a denoising technique.

A closed form solution for this problem is not known and numerous approaches are available to find the minimizer. An extensive comparison of the solvers: Iterative Threshold, Accelerated Proximal Gradient, Augmented Lagrange Multiplier, and Inexact Augmented Lagrange Multiplier can be found in (Lin, Chen et al. 2010), leading to Inexact Augmented Lagrange Multiplier as the faster solver for the same accuracy to the RPCA problem. The latter was selected and used throughout this thesis. It worth mentioning an alternative approach presented by (Becker, Candès et al. 2011) for this problem using TFOCS, a general purpose first-order conic solver, which produces a comparable solution regarding relative error and can be used to prototype other algorithms.

The augmented Lagrange multiplier method when applied to the problem 7.14 gives the following optimization problem:

$$
\min _{L, O}\|L\|_{*}+\lambda\|O\|_{1}+\langle Y, D-(L+O)\rangle+\frac{\alpha}{2}\|D-(L+O)\|_{F}^{2}
$$

Where the third term enforces the relaxed constraint via the matrix of Lagrange multipliers $Y$ and fourth term makes the objective function strictly convex, improving the convergence.

The inexact augmented Lagrange multiplier algorithm (Lin, Chen et al. 2010) which solves 7.16 is presented below: 


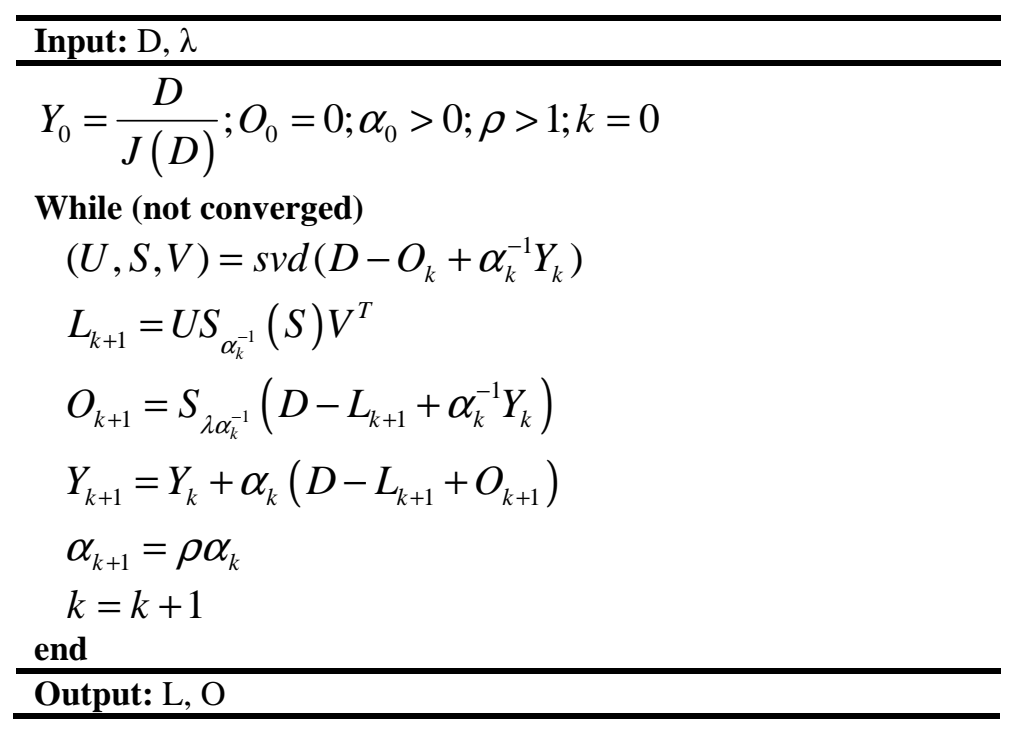

It worth mentioning that in the implementation of this algorithm the parameter $\alpha$ and $\rho$ might need to be tuned.

A synthetic example using a data matrix $\mathrm{D}(100 \times 100)$ formed by a normally distributed low-rank signal L and a uniformly distributed sparse outliers illustrates the presented algorithm working.
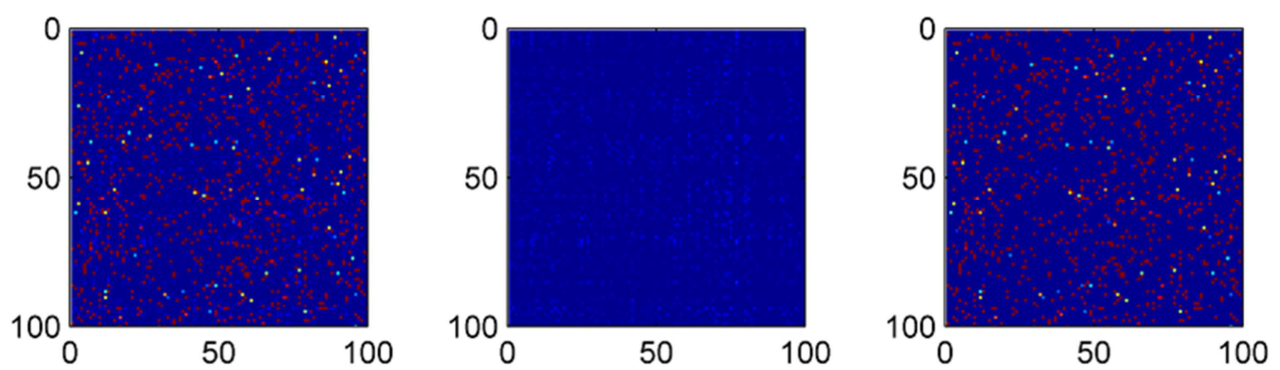

Figure 7.14 - Synthetic example of the inexact augmented Lagrange multiplier RPCA algorithm.

The bounds provided for this example $\operatorname{are} \operatorname{rank}(\mathrm{L})=5$ and $\operatorname{card}(\mathrm{O})=20 \%$. As can be seen in Figure 7.15 at less than 30 iterations it has been converged to the specified tolerance. 

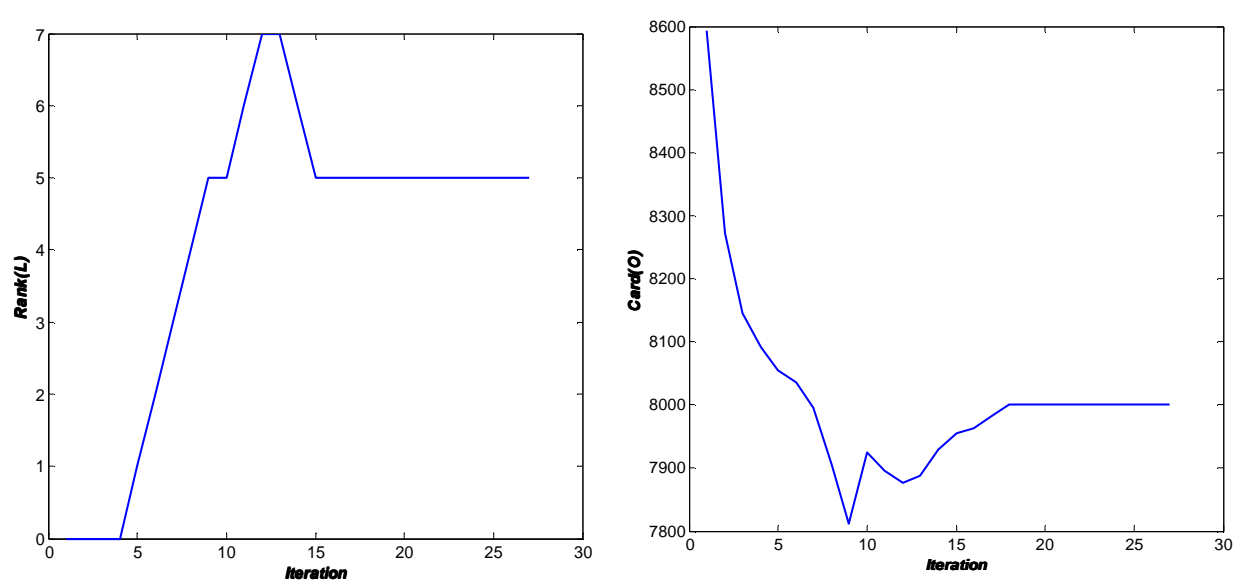

Figure 7.15 - Rank $(\mathrm{L})$ and $\mathrm{Card}(\mathrm{O})$ as a function of iteration.

The relative error as a function of iteration is shown in Figure 7.16. It confirms the convergence of the algorithm in 28 iterations. Notice that the computational cost for this algorithm is higher than the classical PCA but the difference is not expressive. This example in the same computer using MatLab ${ }^{\mathrm{TM}}$ took $0.6 \mathrm{~s}$ against $0.08 \mathrm{~s}$ to run.

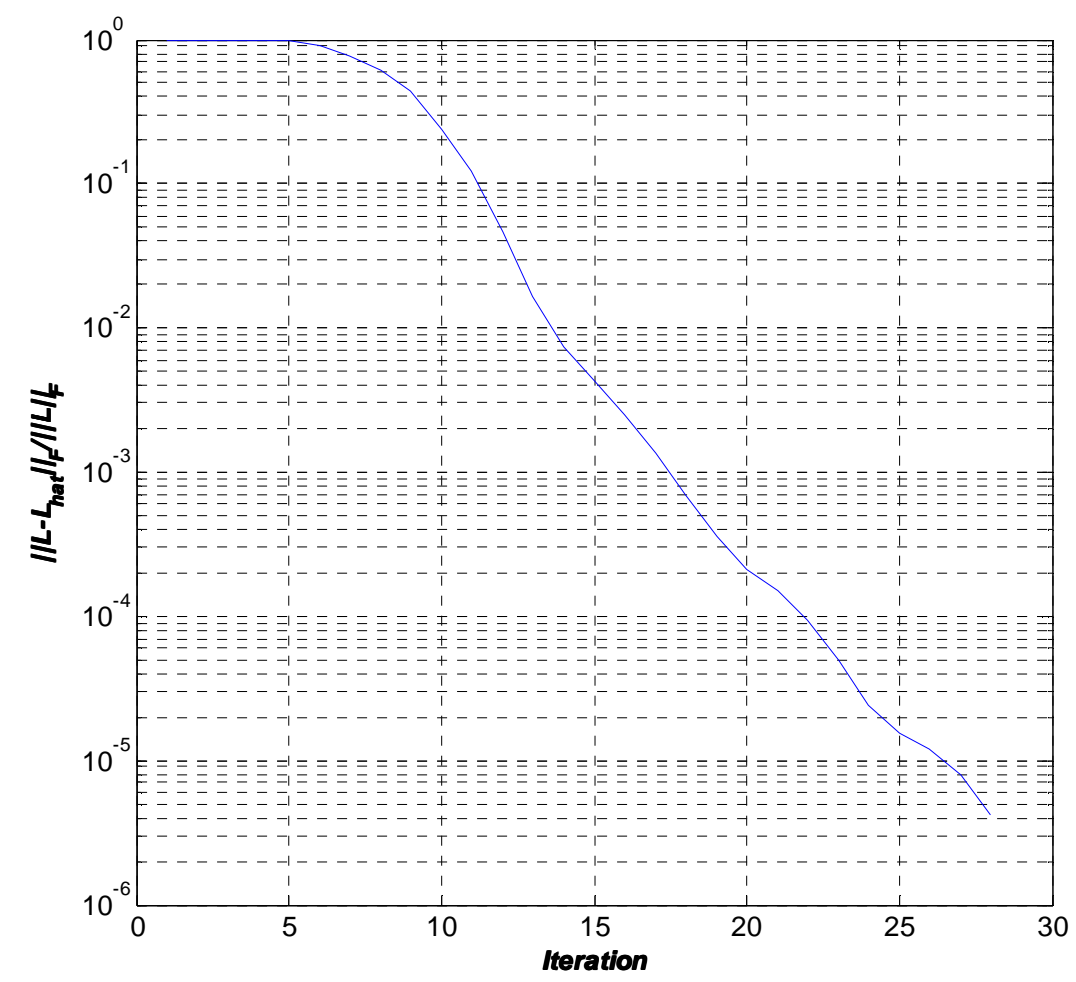

Figure 7.16 - Relative error as function of the iteration. 
In the context of the centralized pre-processing framework the inexact augmented Lagrange multiplier algorithm RPCA is used for the missing data, denoising, and outlier removal procedure. As we are solving the relaxed problem 7.16 a noise level estimator is necessary to correctly define the algorithm tolerance. The robust median noise estimator as defined in (Olsen 2011) is used to solve this problem.

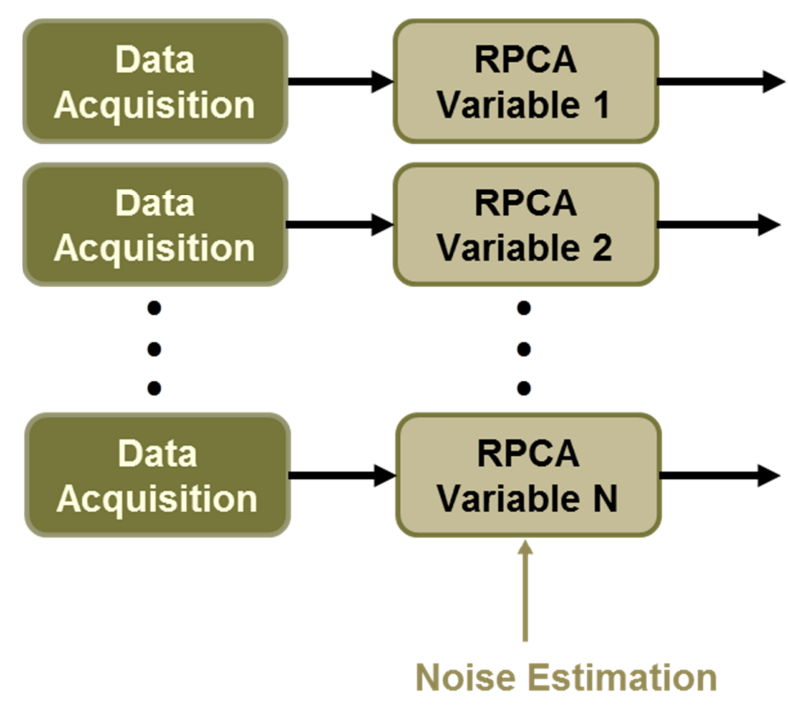

Figure 7.17 - Denoising procedure in the pre-processing framework

\section{3}

\section{Feature Extraction}

A general multi-sensor feature extraction framework based on Diffusion Maps (Coifman and Lafon 2006) is presented in this section. Two important aspects of this method which were extensively studied in (Lafon 2004) are used: density-invariant embedding and extension of a given embedding. In the approach we assume that all sensors are measuring different features of the same physical phenomenon. Since different features might have different characteristic scales, they are chosen empirically during the training stage (training signal embedding). New signal embedding extension is calculated and classified accordingly using KNN (k-nearest neighbor classifier). This completes the general idea of the framework which is explained in the next two sub-sections. 


\subsection{1}

\section{Diffusion Maps}

DM (Coifman and Lafon 2006) is a non-linear dimensionality reduction and clustering technique suitable for non-uniformly distributed datasets. The technique unifies the analysis of spectral clustering and spectral embedding algorithms through a probabilistic framework. The general idea is introduced by a notion of diffusion distance which is the distance of a random walk between two nodes on a graph. Notice that this definition can reveal different local structures at different times. The graph nodes are then non-linearly embedded on the eigenvector coordinates of a normalized graph Laplacian converting the diffusion distance into Euclidean distance in the embedded space. Therefore it gives a probabilistic meaning for this distance which depends on the dataset density and geometry. DM when compared with other techniques is robust to noise and computationally inexpensive for small and medium datasets.

\subsubsection{1 \\ Diffusion Maps Algorithm}

Given a data set $X=\left\{x_{i}\right\}_{i=1}^{n}, x_{i} \in \square^{d}$ we construct a weighted symmetric graph using $x_{i}$ as nodes. A distance between elements $x_{i}$ and $x_{j}$ of the dataset is defined and used to weight the graph edges. A usual choice for the weight function is the Gaussian kernel:

$$
W_{\varepsilon}\left(x_{i}, x_{j}\right)=e^{-\frac{D\left(x_{i}, x_{j}\right)^{2}}{\varepsilon}}
$$

Where

$D$ - distance defined for the dataset, e.g., Euclidean distance;

$\varepsilon$ - characteristic scale (kernel bandwidth).

Afterwards a random walk (diffusion process) is induced by computing a row-normalized Markov matrix (transition probability matrix) taking into account the influence of the density which gives the new kernel:

$$
W_{\varepsilon, \alpha}\left(x_{i}, x_{j}\right)=\frac{W_{\varepsilon}\left(x_{i}, x_{j}\right)}{q_{\varepsilon}\left(x_{i}\right)^{\alpha} q_{\varepsilon}\left(x_{j}\right)^{\alpha}}
$$

Where

$$
q_{\varepsilon}\left(x_{i}\right)=\sum_{j} W_{\varepsilon}\left(x_{i}, x_{j}\right)
$$


$\alpha=0$ means maximal density influence (graph Laplacian operator)

$\alpha=1$ means minimal density influence (Laplace-Beltrami operator)

A symmetric normalization is applied to the new kernel preparing for spectral decomposition which enables the geometric structure of the dataset to be studied in a compact feature space.

$$
W_{s y m}\left(x_{i}, x_{j}\right)=\frac{W_{\varepsilon, \alpha}\left(x_{i}, x_{j}\right)}{v\left(x_{i}\right)^{0.5} v\left(x_{j}\right)^{0.5}}
$$

Where

$$
v_{\varepsilon, \alpha}\left(x_{i}\right)=\sum_{j} W_{\varepsilon, \alpha}\left(x_{i}, x_{j}\right)
$$

A complete sequence of eigenvectors and eigenvalues of $W_{\text {sym }}$ are available, satisfying:

$$
\left\{\begin{array}{c}
W_{s y m} \psi_{l(t)}(x)=\lambda_{l(t)}^{t} \psi_{l(t)}(x) \\
1=\lambda_{0}>\left|\lambda_{1}\right| \geq \ldots \geq\left|\lambda_{l(t)}\right|
\end{array}\right.
$$

Where $l$ (new space dimensionality) is chosen based on a specified tolerance $\delta$

$$
\frac{\left|\lambda_{l}^{t}\right|}{\left|\lambda_{1}^{t}\right|}>\delta
$$

The diffusion distance approximation can be interpreted as the Euclidean distance (Coifman and Lafon 2006):

$$
D_{t}^{2}\left(x_{i}, x_{j}\right) \square \sum_{k=1}^{l(t)} \lambda_{k}^{2 t}\left(\psi_{k}\left(x_{i}\right)-\psi_{k}\left(x_{j}\right)\right)^{2}=\left\|\Psi_{t}\left(x_{i}\right)-\Psi_{t}\left(x_{j}\right)\right\|^{2}
$$

Where

$$
\Psi_{t}=\left[\lambda_{1}^{t} \psi_{1}\left(x_{i}\right), \ldots, \lambda_{l}^{t} \psi_{l}\left(x_{i}\right)\right]^{T} \text { for some } t>0 \text { is the DM of the dataset } x_{i}
$$

into the Euclidean space $\square^{l}$.

\subsubsection{2}

\section{Toy Example}

A Trefoil Knot with Gaussian noise is used as a representative example of the potential of the DM as a clustering algorithm. The data set $\mathrm{X}$ is threedimensional with 400 data points (Figure 7.18): 


$$
X=\left\{\begin{array}{c}
x=\sin (3 t)+r \\
y=\sin (t)+2 \sin (2 t)+r \\
z=\cos (t)-2 \cos (2 t)+r
\end{array}\right.
$$

Where

$$
0 \leq t \leq 2 \pi
$$

$r \square N(0,0.05)$

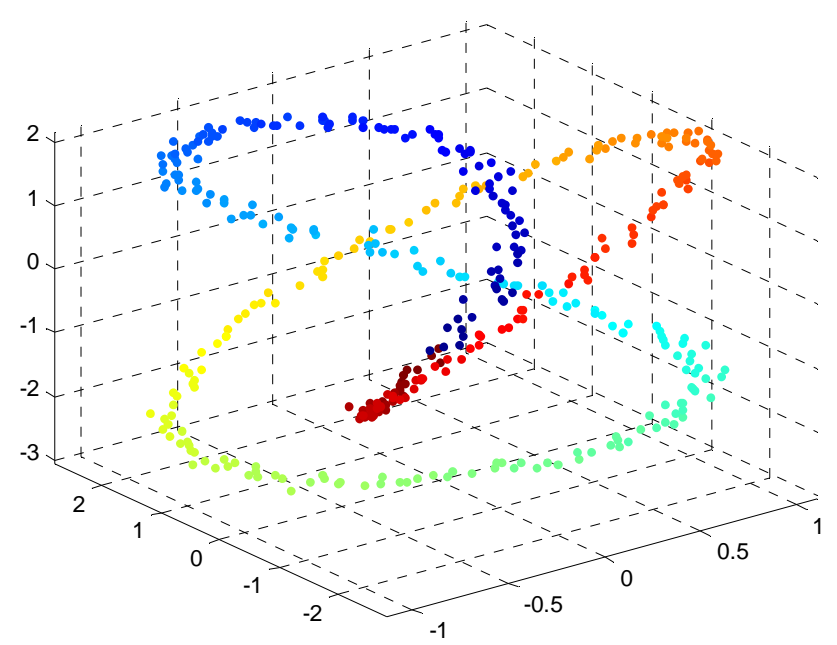

Figure 7.18 - Trefoil knot with Gaussian noise.

The Gaussian Kernel is assembled using Euclidean distances to every point in the data set. Color is only used to indicate different data which means that an additional dimension for color is not used. The resulting DMs for Trefoil using the first two eigenvalues $\left(\lambda_{1}\right.$ and $\left.\lambda_{2}\right)$ and kernel bandwidth of 0.35 can be seen in Figure 7.19. It shows that in the diffusion space the Trefoil was completely untied, meaning all similar data is represented together. In addition, the algorithm on an $h p$ notebook equipped with an i5 processor and using MatLab ${ }^{\mathrm{TM}}$ took 0.266 $\mathrm{s}$ to run. 


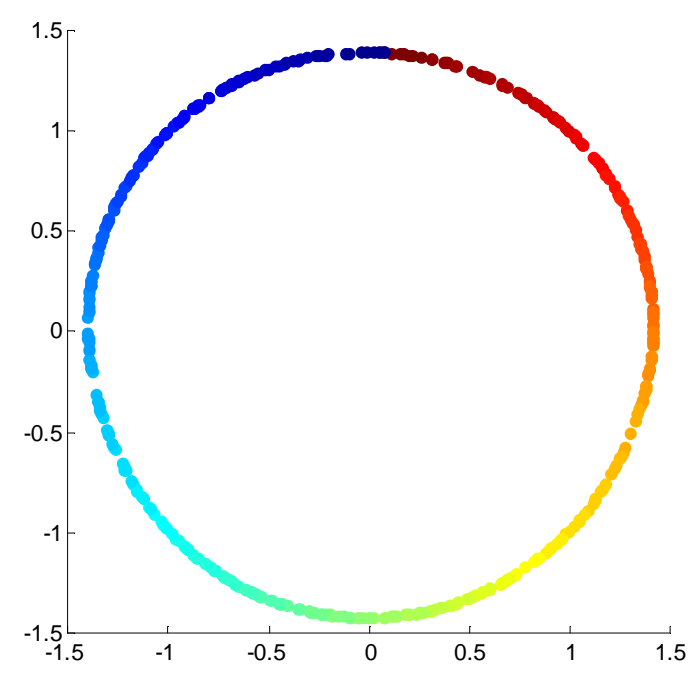

Figure 7.19 - Trefoil knot with Gaussian noise.

\subsection{2}

\section{Feature Extraction Framework}

The aim of our feature extraction framework is to capture the underlying and mutual influences of the production processes in the context of integrated production framework. The multi-sensor is based on the spectral and density invariant embedding of the DMs framework using Mahalanobis distance. It is appropriate to multivariate analysis for the training stage and allows the DMs to be computed simultaneously for the different signals. Each scale parameter $\varepsilon_{i}$ for each required feature $i$ of the signal is chosen based on the Singer Measure (Coifman and Lafon 2006):

$$
S M\left(\varepsilon_{i}\right)=\sum_{j} e^{-\frac{D^{2}\left(x_{i}-x_{j}\right)}{\varepsilon_{i}}}
$$

This measure can be interpreted as an $s$ shape curve where the extremities represent a non-connected graph and a Markov chain at steady state. As recommended by (Coifman and Lafon 2006) the optimal value of the scale parameter is chosen as the midpoint of the linear part of the $s$ shape curve.

Nyström extension as proposed by (Lafon 2004) is used to compute the embedding coordinates for the new signal. The result is then classified by kNearest Neighbors (K-NN). The framework is presented in Figure 7.19. 


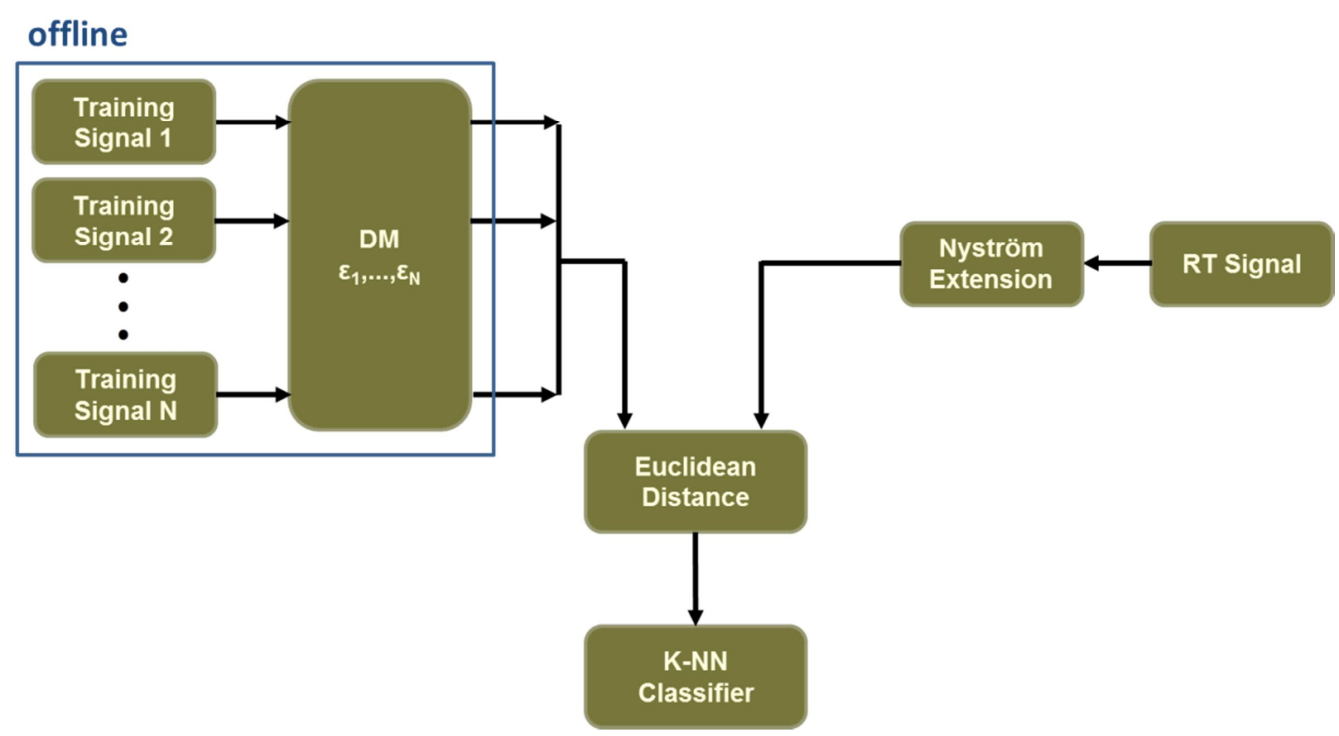

Figure 7.20 - Trefoil knot with Gaussian noise.

A multi-sensor framework which was introduced and tested by (Keller, Coifman et al. 2010) on an audio-visual speech recognition application might be also explored for real-time production signals. It uses similar ideas of the work by (Lafon 2004) and seems to be more general than our framework. In this work each signal is embedded separately and then appended to the same coordinates system. Different distances might be used with the weight functions for each different signal by using SHD (Symetric Hausdorff Distance) at the embedded space which deals with the matching and alignment requirements that may occur. Once again a simple classifier as k-NN is used. The evaluation of this technique is considered a future work in this thesis.

\section{4 \\ Pre-processing Framework}

The integrated production and optimization framework calls for multisensor data analysis and fusion to recover underlying processes and mutual influences through a more informative and less application dependent data analysis approach. In this context a centralized pre-processing (Figure 7.17) less sensitive to noise, outliers, missing data, and able to deal with time synchronization, compression/decimation/data reduction, and feature extraction is a goal to pursue as presented in Chapter 6 . We propose to use the algorithm presented in section 7.2 to deal simultaneously with noise, missing data, and outliers, and the algorithm presented in section 7.3 to deal with feature extraction. 
Time synchronization and compression/decimation were not included in the centralized pre-processing framework proposed in this thesis. They are considered a future work.

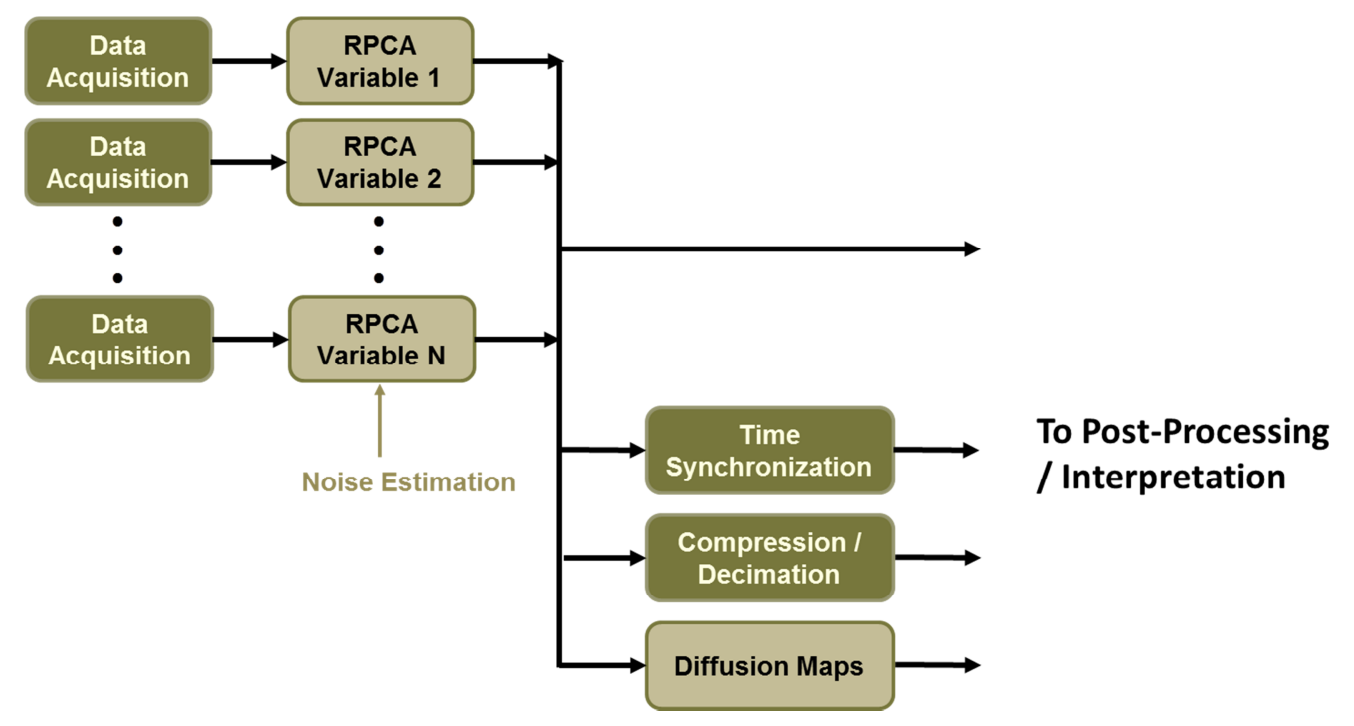

Figure 7.21 - Centralized pre-processing framework.

Time synchronization has to deal with time lags as a consequence of the different low level time references and networked time synchronization issues. Efforts to solve the time synchronization problem using open standards can be found in JIPs as SIIS and IWIS but they still far from a solution. At the network level a recent standard IEEE-1588 (Standard Precision Time Protocol) which is a replacement for the NTP (Network Time Protocol) is an important advance for distributed systems in automation technology. Distributed clocks can be synchronized, e.g., with an accuracy of less than 1 microsecond via Ethernet networks with low demands on the local clocks and the network and computing capacity. From the data analysis point of view time synchronization impact on the multivariate advanced correlation and analysis. Time series analysis has been successfully used by (Aggrey 2007) to solve data alignment problem in multiple IW data. We see this problem as part of the centralized pre-processing framework using clustering algorithms to deal with the data alignment problem. It is worth mentioning that it is not a replacement for the low level time synchronization. Sparse subspace clustering (Elhamifar and Vidal 2009) might be an option for a clustering algorithm to deal with this problem.

Compression/decimation/data reduction is always an issue for monitoring systems in subsea production environment due to limited bandwidth, storage, and 
computing capacity. In addition, the data overload due to the increasing number of sensors make this problem even worse as mentioned in Chapter 6. On the other hand, data analysis might be impacted by these algorithms especially by the lossy ones. The most frequent approach used is taking less number of measurements by removing the ones with no significant information content. It is accomplished by discarding measurements with assumed small variations from the time intervals. This task is completed through a specified threshold and it is signal dependent. Notice that data analysis algorithms which need equally spaced measurements as, e.g., PCA and Waveshrink are impacted by this approach. We see this problem as a change in the data acquisition by using compressive sensing (Candès, Romberg et al. 2006) where the signal is acquired compressed and can be reconstructed lossly, solving the problem. It is worth mentioning that in this case the denoising and outlier removal proposed in this text should be different.

The following chapter describes the work by (Olsen 2011) regarding preprocessing techniques which is used as a reference work for validation purposes. Pressure and temperature data generated by the non-isothermal dynamic IWreservoir model developed are corrupted by noise and outliers and used as the input for both approaches. Only pressure is really compared for denoising/outlier removal and transient identification. Transient temperature data is not available in the literature or in Olsen's work. Case studies complete the chapter showing the results. 


\section{8 \\ Pre-processing Framework Validation and Case Studies}

The Chapter 8 is divided in: signal generation, validation and case studies. Signal generation presents the synthetic pressure and temperature signals generated from the noise/outlier model used and the one developed in Chapter 4. Validation is accomplished through the comparison with the results from the work by (Olsen 2011) which has an extensive study of automatic filtering and transient identification on real-time transient pressure signals. Our feature extraction approach was applied for transient identification but as described earlier it is general and can be used for different applications. Finally, case studies evaluate denoising/outlier removal and transient identification on transient temperature signals, and transient identification on both. It is worth mentioning that temperature transient analysis is limited and considered in early stage of development (Muradov and Davies 2011). In addition, there are almost none real data available in the literature for IW applications.

\section{1 \\ Noise, Outlier, and Signals}

Transient signals in real wells might be generated due to shut-ins or zonal shut-ins in case of IWs. They can be used in context real-time production monitoring as an opportunity well-test. Automatic filtering and transient detection for this application provide the appropriate pre-processing to be used for well-test analysis enabling more frequent tests and consequently more information to optimize reservoir management.

Real pressure and temperature signals in a dual zone injector IW and its producer pair can be seen in the Figures 8.1 and 8.2 respectively. Notice that only the lower zone was closed creating a transient. Even with the graph scale used it is possible to see the noise and the outliers. It is a subsea well with a water depth of $1168 \mathrm{~m}$ and downhole sensors installed at $2644.1 \mathrm{~m}$ and $2656.5 \mathrm{~m}$ (TVD). 


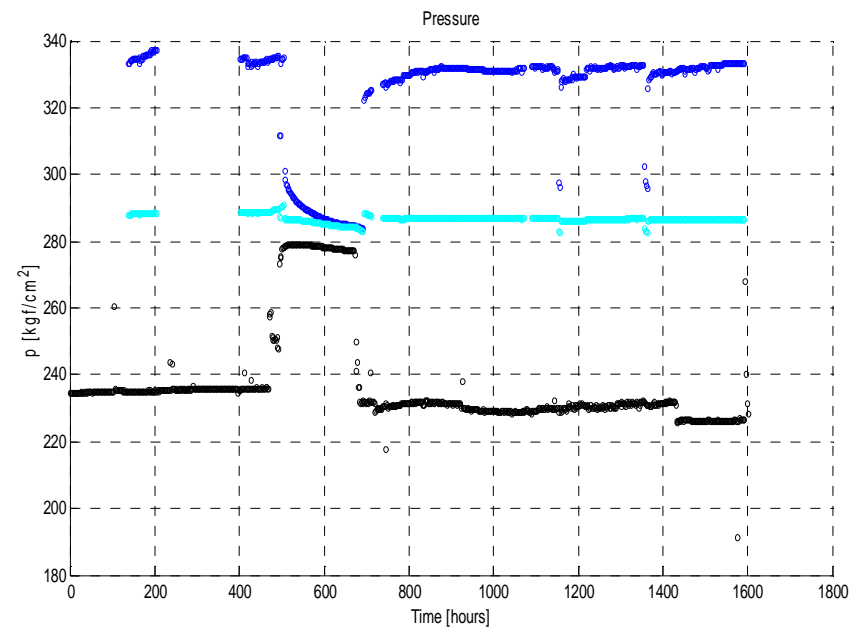

Figure 8.1 - Injector-producer pair pressure signal (dark blue and light blue are lower and upper zones respectively; black producer well).

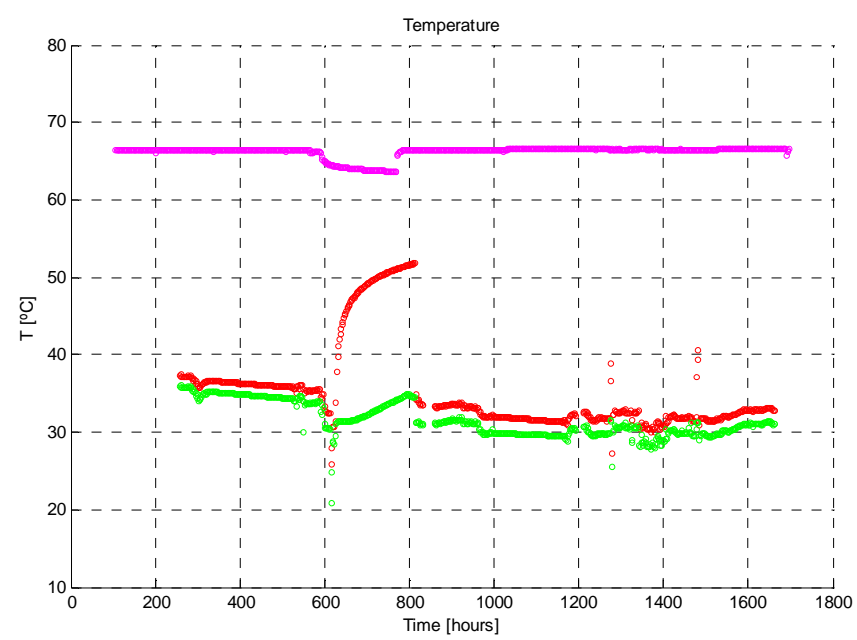

Figure 8.2 - Injector-producer pair temperature signal (red and green are lower and upper zones respectively; pink producer well).

The pressure and temperature signals used in the validation and case studies in this thesis were generated synthetically from the Chapter 4 model. It is assumed that those signals are representative of the ones measured in an IW. We choose to generate synthetic signal for two main reasons: the lack of transient pressure and temperature signals available in the literature for IWs and the complete control over the true noise level used in these signals. To be fair with the comparisons made against Olsen's work we do not generate rate changes with less than 10 measurements, the time interval is kept under 3-360 seconds, noise distribution is 
normal with mean zero and variance within 1 to 10 , and the outliers distribution is uniform with level within 5 to 100 times the noise level.

Three different synthetic sets of pressure and temperature signals were generated as representative signals from real IWs. They are corrupted by noise and outliers and the same amount of both are added in each set of pressure and temperature signals as they come from the same sensor. All three sets were generated by 10 rate changes at random within 2000 to $500 \mathrm{bpd}$. Each signal has sample rate of 6 minutes and 27000 measurements. Outliers were chosen from uniform distribution with level varying from 10 to 100 times of the noise level and number within $0.3 \%$ to $5 \%$ of the total number of measurements and noise is assumed to be additive white Gaussian noise with variance within 1 to 6 . Partial pressure and temperature of signals 1,2 and 3 can be seen in Figure 8.3.
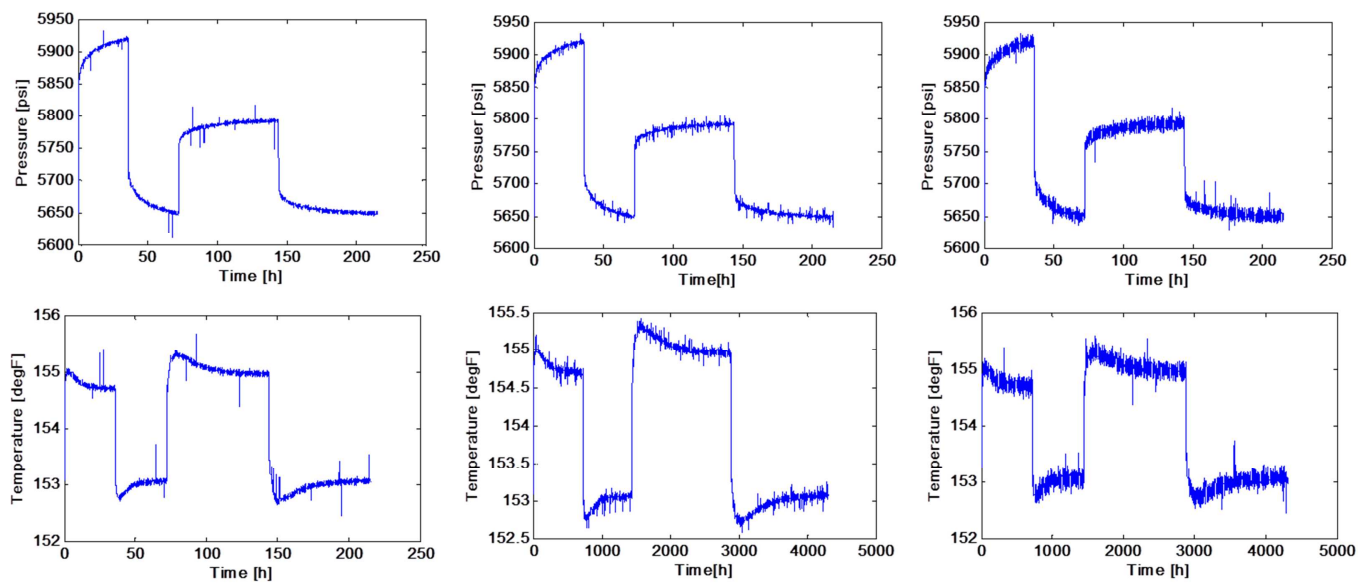

Figure 8.3 - Pressure and temperature signals 1,2 and 3 .

The quality of the filtering is measured by the RMSE (Root Mean Squared Error) divided by the noise level estimate (standard deviation). This metric was introduced by (Olsen 2011) to compare different de-noised signals with different noise levels. Outlier removal and transient identification performance are measured by the percentage of success in the removal and in the identification after the outlier removal/denoising procedures. It is considered an error when the identification occurs after three measurements from where it actually happens.

$$
R M S E_{\sigma}=\frac{1}{\sigma} \sqrt{\frac{1}{N} \sum_{t}\left(y_{t}-y_{t, \text { clean }}\right)^{2}}
$$


Where

$\sigma-$ noise standard deviation.

\section{2}

\section{Denoising/Outlier Removal and Transient Identification}

The wavelet analysis to denoise pressure signals and detect transients was first introduced by (Kikani and He 1998). Since then, a lot of work have been done such as, e.g., the work by (Athichanagorn, Horne et al. 1999) which introduced the seven step procedure for processing data from downhole sensors, the work by (Ribeiro, Pires et al. 2008) which showed that there is no universal wavelet transform to be applied in any dataset due to strong dependence on decomposition level and wavelet type, and the work by (Olsen 2011) who did an extensive study on automatic outlier removal, denoising, data reduction, and transient identification on pressure and rate signal for well test analysis. The latter addressed the problem presented by (Ribeiro, Pires et al. 2008) and was used as reference work to ours for validation purposes.

\subsection{1}

\section{Denoising/Outlier Removal}

The work by (Olsen 2011) developed a method to detect and remove multiple outliers without requirements regarding the signal considered. The outliers are detected and removed by a median filter with noise estimation from the Waveshrink method. The latter is used afterwards for conventional denoising purposes. In the opinion of the author, this method addresses the two main drawbacks found in wavelet analysis in the petroleum industry literature: single outlier removal and dependence upon the signal considered. The idea is schematically illustrated in Figure 8.5.

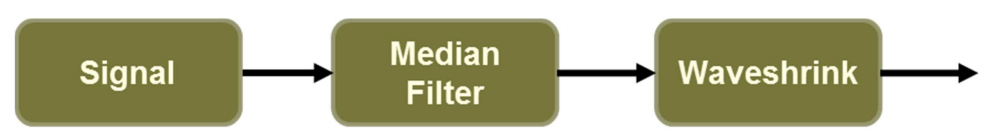

Figure 8.4 - Outlier removal and denoising in Olsen's Work.

The median filter considered is a moving window filter with a window size of $n$ measurements which is usually small compared to signal measurements. If 
the value in the middle of the window is higher than the median plus a given threshold, it will be considered an outlier and therefore removed. The threshold is calculated using the noise estimator proposed in (Donoho and Johnstone 1994) as being the MAD (Median Absolute Deviation) divided by 0.6745 which is evaluated at the first decomposition level in the wavelet transform but using only the absolute values of the non-zero detail coefficients. In Olsen's work it is called a RMAD divided by 0.6745 to make it different from the original. Mathematically, the median filter can be defined as:

$$
y_{t, \text { median }}= \begin{cases}y_{t} & \left|\operatorname{median}(w)-y_{t}\right| \leq 3 \sigma \\ - & \left|\operatorname{median}(w)-y_{t}\right|>3 \sigma\end{cases}
$$

Where:

$$
\begin{aligned}
& \sigma=\mathrm{RMAD} / 0.6745 \\
& w \text { - window size. }
\end{aligned}
$$

Notice that the maximum window size recommended is 19 measurements from which 9 outliers can be removed $\left(\frac{w-1}{2}\right)$ and it is empirically defined in the scope of the well-test analysis (Olsen 2011).

The Waveshrink method was selected as the denoising filter for pressure and rate signals since wavelet analysis was introduced to well-test analysis by (Kikani and He 1998). However, only with the work by (Pico, Aguiar et al. 2009) and (Olsen 2011) an extensive study was done regarding wavelet selection, shrinkage rule, threshold estimator, noise estimator, and primary resolution level to choose. The best selection for the Waveshrink method in Olsen's work were based on average results of the smallest error in the root-mean-squared sense between the clean signal and the noisy signal for more than 500 synthetic pressure or rate signals. The characteristics of those signals were chosen as representative ones from real-time pressure and rate signals gathered from North Sea wells.

The Waveshrink method (Donoho and Johnstone 1994) is based on the principle of shrinking the wavelet coefficients towards zero in order to remove noise. The main idea is to transform signal $y_{t}$ to the wavelet domain, shrink its empirical wavelet coefficients towards zero, and transform the shrunk coefficients back to the signal domain. Mathematically, it can be stated as: 
$\hat{y}_{t}=W^{-1}\left(D\left(W\left(y_{t, n o i s e}\right), \lambda\right)\right)$

Where:

$W$ - wavelet transform;

$D$ - shrinkage operator;

$\lambda$ - threshold function;

$W^{1}-$ inverse wavelet transform.

The shrinking operator might be hard, soft or a function and operates on the wavelet coefficients through a threshold estimator $\lambda$ which is function of the noise and the resolution level considered. In Olsen's work the best options for pressure and rate signals in the scope of the well test analysis are:

Bi-orthogonal spline39 wavelet for pressure (Figure 8.6 and Table 8.1):
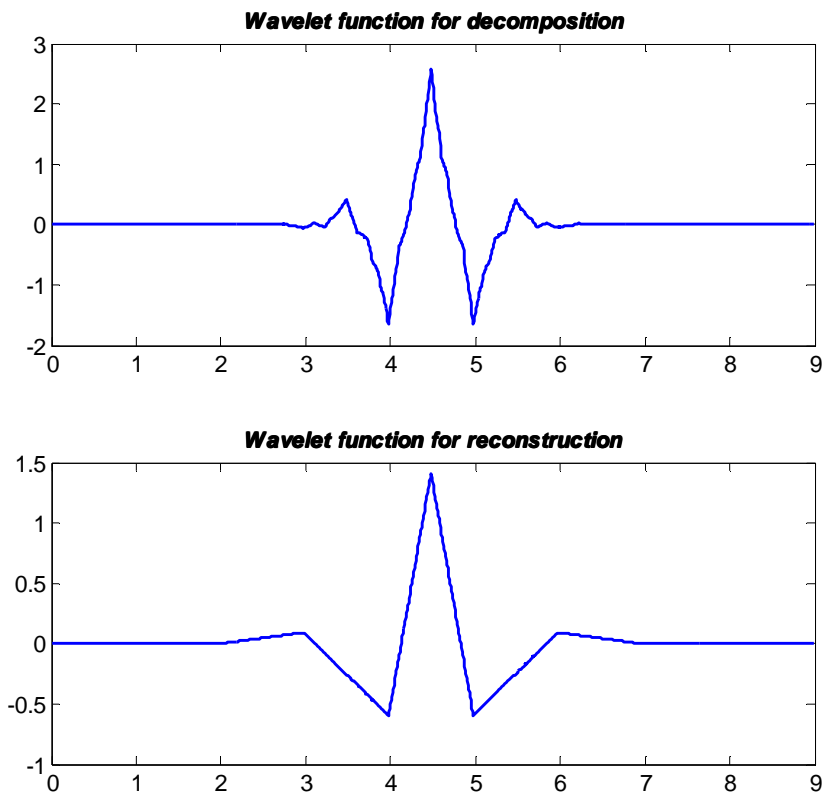

Figure 8.5 - Bi-orthogonal Spline39 wavelet functions.

\begin{tabular}{|l|l|l|l|l|l|l|l|l|l|}
\hline & 0 & 1 & 2 & 3 & 4 & 5 & 6 & 7 & 8 \\
\hline$h(N)$ & $\frac{3}{128}$ & $\frac{-3}{64}$ & $\frac{-1}{8}$ & $\frac{19}{64}$ & $\frac{45}{64}$ & $\frac{19}{64}$ & $\frac{-1}{8}$ & $\frac{-3}{64}$ & $\frac{3}{128}$ \\
\hline$\tilde{h}(N)$ & & & & $\frac{1}{4}$ & $\frac{1}{2}$ & $\frac{1}{4}$ & & & \\
\hline
\end{tabular}

Table 8.1 - Bi-orthogonal Spline39 scaling filters. 
Non-orthogonal spline4246 wavelet for rate (Table 8.2):

\begin{tabular}{|l|l|l|l|l|l|l|}
\hline & 0 & 1 & 2 & 3 & 4 & 5 \\
\hline$h(N)$ & & $\frac{1}{80}$ & $\frac{3}{8}$ & $\frac{3}{8}$ & $\frac{1}{80}$ & \\
\hline$\tilde{h}(N)$ & & & $\frac{-1}{2}$ & $\frac{1}{2}$ & & \\
\hline$g(N)$ & $\frac{-1}{32}$ & $\frac{-7}{32}$ & $\frac{-11}{16}$ & $\frac{11}{16}$ & $\frac{7}{32}$ & $\frac{1}{32}$ \\
\hline$g(N)$ & & $\frac{1}{80}$ & $\frac{3}{8}$ & $\frac{3}{8}$ & $\frac{1}{80}$ & \\
\hline
\end{tabular}

Table 8.2 - Non-orthogonal Spline4246 scaling and wavelet filters.

Shrinkage operator: SCAD (Fan and Li 2001):

$$
D_{\lambda}=\left\{\begin{array}{lr}
\operatorname{sgn}\left(d_{j}\right) \max \left(\left|d_{j}\right|-\lambda, 0\right) & \left|d_{j}\right| \leq 2 \lambda \\
\frac{(3.7-1) d_{j}-3.7 \operatorname{sign}\left(d_{j}\right) \lambda}{1.7} & 2 \lambda<\left|d_{j}\right| \leq 3.7 \lambda \\
d_{j} & \left|d_{j}\right|>3.7 \lambda
\end{array}\right.
$$

Where

Threshold function: $\sigma(-0.51 j+3.25), j=1, \ldots, 6(j$ is the resolution level);

Noise estimation: $\sigma=\frac{R M A D}{0.6745}$

Minimum resolution level recommended: $j_{0}(N)=\log _{8}(N)-1(N$ is the number of measurements).

Unequally spaced signal measurements are normally required to be denoised in real pressure and rate measurements. Wavelet analysis assumes equally spaced signal measurement to achieve its full error performance. It is recommended in Olsen's work to assume equally spaced signal measurements even when they are not in the scope of the well test analysis. The main reason indicated is to avoid the interpolation of the signal measurements which tends to magnify noise structures present in the signal measurements. 


\section{2 .2}

\section{Transient Detection}

In the literature there are a variety of transient detection methods. The majority of them are based on pattern recognition, regression, threshold or wavelet methods. The transient detection presented in this section describes the approach used by (Olsen 2011) for automatic pressure transient detection in the scope of well-test analysis. It is based on a mask-matching pattern recognition approach performed right after outlier removal and denoising procedures (Figure 8.7).

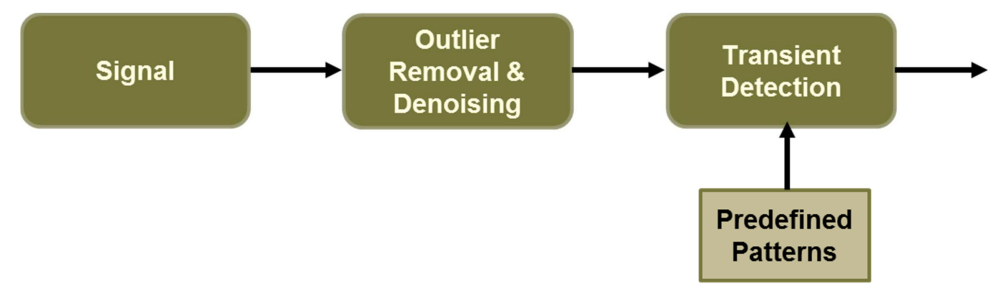

Figure 8.6 - Transient detection approach based on mask-matching pattern recognition.

A transient is detected if the signal window, which is supposed to represent a transient taken from the real-time measurements, fits entirely inside a predefined mask. Four masks representing a build-up with prior pressure increase and decrease and a drawdown with prior pressure increase and decrease patterns are available. They were generated by considering the synthetic and real pressure signal used in the work. The windows are 20 measurements wide (10 measurements before and 10 measurements after the beginning of the transient) and 20 pressure values height which means a vertical resolution of $P_{\max }-P_{\min } / 20$. In order to ensure the identification performance, the pressure difference should be at least three times the estimated noise level $(3 \sigma)$ with standard deviation of at least 0.1 of the entire signal standard deviation.

\section{3}

\section{Validation Using Only Pressure Signal}

The validation of the RPCA denoising/outlier removal technique is performed comparing the result with the median filter/Waveshrink technique for the three pressure signals presented in section 8.1. Notice that set 1 is an average signal, set 2 is worse regarding outliers (level and number), and set 3 is worse regarding noise (level). The parameters used for the later were: Spline39 wavelet, 
decomposition level 5, SCAD as shrinkage operator and threshold $0.7 \sigma_{j}(\mathrm{j}=1, \ldots, 5)$. Transient identification uses the same approach comparing the performance of DM technique with the mask matching technique for the three pressure signals. As discussed earlier it is considered an error for the DM technique when the identification occurs after three measurements from where it actually happens or when the identification do not fit entirely inside the predefined mask for the maskmatching technique. All results are summarized at Table 8.3.
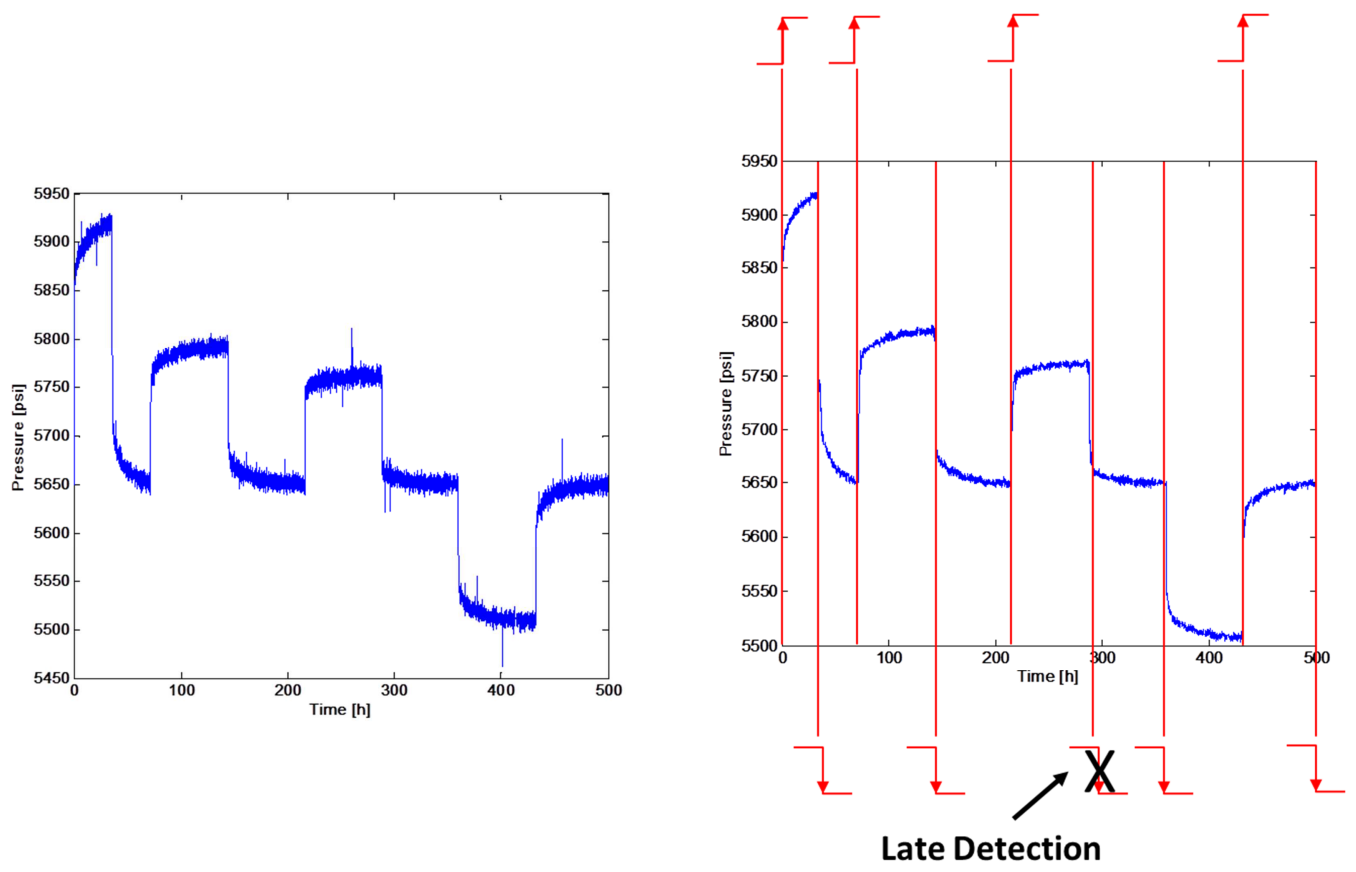

Figure 8.8 - Denoising/outlier removal and transient identification results using pressure signal.

\begin{tabular}{|c|c|c|c|c|}
\hline & RPCA & $\begin{array}{c}\text { Median } \\
+ \\
\text { Waveshrink }\end{array}$ & DM & $\begin{array}{c}\text { Mask } \\
\text { Matching }\end{array}$ \\
\hline Signal 1 & 0.229 & 0.389 & $80 \%$ & $75 \%$ \\
\hline Signal 2 & 0.157 & 0.418 & $90 \%$ & $70 \%$ \\
\hline Signal 3 & 0.481 & 0.621 & $75 \%$ & $70 \%$ \\
\hline
\end{tabular}

Table 8.3 - Denoising/outlier removal and transient identification performance results using pressure signal.

Notice that our approach only uses the noise estimate and the assumption of sparse outliers and low rank signal to denoise and outlier removal. Even for 1D signal it is possible adequately use the technique by using time slices of the signal 
as columns of the data matrix (low rank matrix by construction). For multidimensional signals the same approach is used for each dimension forming at least a block-sparse matrix. Missing data is not an issue and all of them are treated simultaneously. Transient identification has a drawback due to training process common to a pattern recognition algorithm.

\section{4}

\section{Case Studies}

We divided the case studies in two types. The first one shows that the temperature signal can be used in the same way as the pressure signal in transient detection. The other concludes that denoising/outlier removal and feature extraction methods are appropriate for multidimensional signals of multiple sensors as can be found in IW applications. Note that the great advantages of the proposed methods are the possibility of treating nonuniform data and the no need of a previous knowledge of the signal but the noise level. The computational performance of these methods is reasonable and very comparable to the reference ones. However, a more detailed study is needed, mainly for medium and high dimensions data.

\subsection{1}

\section{Denoising and Outlier Removal of Transient Temperature Signals}

The goal of this case study is showing that isolated temperature signal can be used in the correct transient detection. The temperature signals used are the same as the ones described in section 8.1. They are first filtered and afterwards used in the transient detection. The filter uses only the noise level estimator, the feature extraction method, which was trained with temperature transient signals, and the K-NN classifier that required order 6 . The collected results are shown in Table 8.4. 

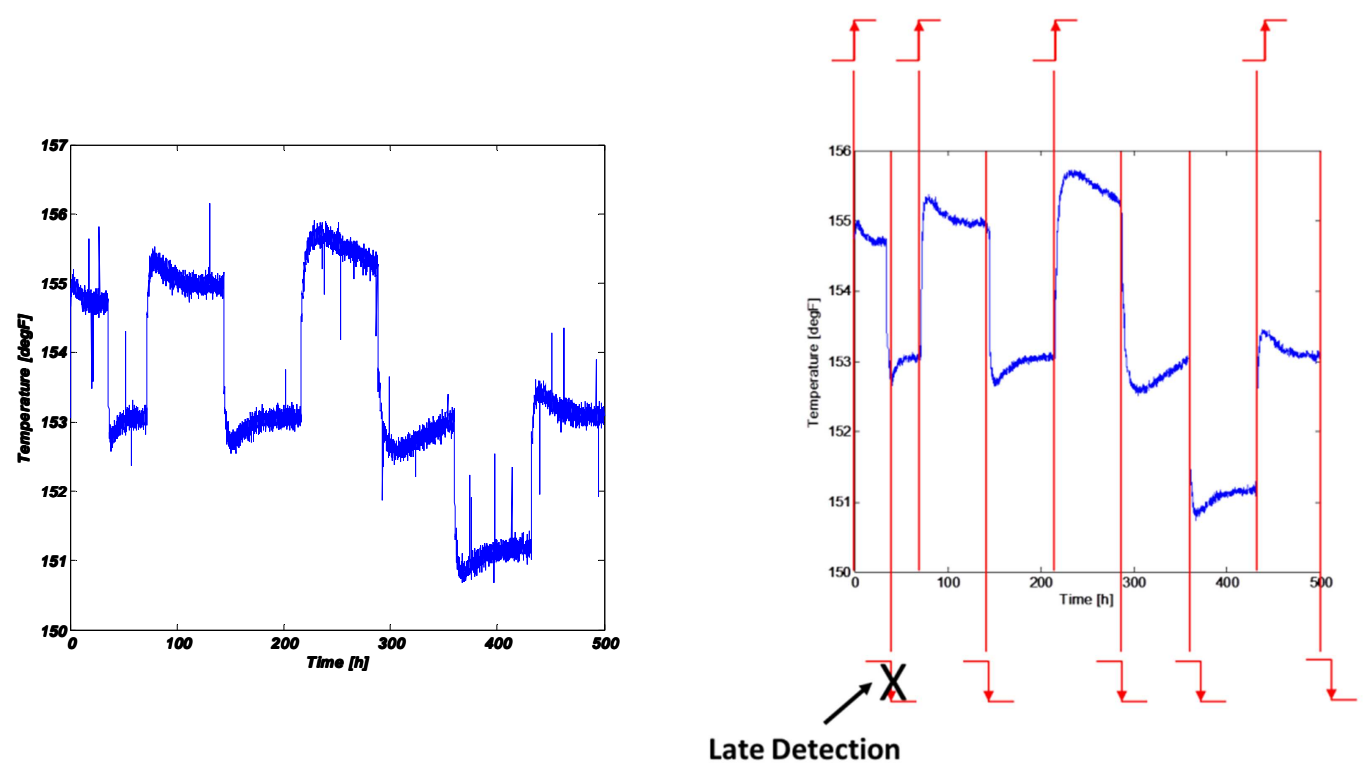

Figure 8.9 - Denoising/outlier removal and transient identification results using temperature signal.

\begin{tabular}{|l|l|l|}
\hline & RPCA & DM \\
\hline Signal 1 & 0.439 & $80 \%$ \\
\hline Signal 2 & 0.259 & $85 \%$ \\
\hline Signal 3 & 0.738 & $70 \%$ \\
\hline
\end{tabular}

Table 8.4 - Denoising/outlier removal and transient identification results using temperature signal.

\subsection{2}

\section{Transient Detection through Pressure and Temperature Signals}

Transient detection was tested with the three synthetic temperature signals described in section 8.1. They were filtered as in the previous study case and the results can be seen in Table 8.5. If we compare the results with the isolated pressure and temperature results we can see an improvement in the percentage of correct detection due to the additional dimension, in other words, the use of the pressure signal in the detection process. Note that we can include the spatial dimensional when distributed and quasi-distributed sensors are used. In this case, nonuniform spatial distribution of sensors and nonuniform sample rate are not a problem for the proposed algorithms. The end results for this case can be seen in Table 8.5. 


\begin{tabular}{|c|c|c|}
\hline & RPCA & DM \\
\hline Signal 1 & $0.229 / 0.439$ & $90 \%$ \\
\hline Signal 2 & $0.157 / 0.259$ & $95 \%$ \\
\hline Signal 3 & $0.481 / 0.738$ & $80 \%$ \\
\hline
\end{tabular}

Table 8.5 - Denoising/outlier removal and transient identification performance results using pressure and temperature signals. 


\section{9 \\ Conclusions and Future Works}

Integrated production optimization calls for revisiting the way we deal with the multiple pieces of the petroleum production puzzle. The system view perspective should lead to better production system behavior prediction and fast response to unexpected events. IW and monitoring systems in this perspective might improve both if an adequately use of the technology available is performed. In this thesis we focused on modeling and data analysis pre-processing for well test applications as one example IW monitoring systems in the context of integrated modeling and optimization framework. We address the problem of IW monitoring system design, a fully coupled non-isothermal dynamic IW-reservoir model, and partially the data analysis pre-processing framework through denoising/outlier removal and feature extraction techniques. In the following sections we summarize our achievements and the work which still to be done.

\section{1 \\ IW Monitoring Systems Design}

A method to design IW monitoring systems for the integrated modeling and optimization framework has been developed. It was applied to an IW application using reactive control strategy and artificial lift as a representative example. The main objective was to introduce interpretation model uncertainty as constrain to adequately specify a fit for purpose IW monitoring system. A detailed quantitative study for different applications is certainly a work to be done.

\section{2}

\section{Fully Coupled Non-Isothermal Dynamic IW-Reservoir Model}

A fully coupled dynamic non-isothermal two-phase well-reservoir numerical model for vertical IWs and multilayer reservoirs in the scope of well test analysis has been developed. The model was successfully applied to multizone temperature transient analysis of well total and zonal rate change. The numerical studies showed that transient temperature due to zonal rate change without closing is achievable in a multilayered reservoir. Thermal conduction and crossflow between layers were included in the model but their impact was not independently presented in case studies. Even though the heat transfer coefficients 
allow the use of gas-liquid mixtures in the drift-flux model it was not completely tested. Wellbore storage effect was neglected in our model since our study is limited to the reservoir drainage area but real data provided by (Valiullin, Ramazanov et al. 2009) showed that it can hide the early time effect of the transient temperature. Inclusion of time dependent equations for the IW will bring better understanding of fast transients but it is also a future work.

\section{3}

\section{Data Analysis Pre-Processing}

A new data analysis approach to IW applications with centralized preprocessing has been developed. Denoising and feature extraction techniques were applied to the transient pressure generated by the model developed in this thesis and compared with a reference study by (Olsen 2011). Transient temperature was introduced in this context showing similar results regarding denoising/outlier removal and transient identification. The techniques applied showed satisfactory results for multi-sensor applications. As general techniques they can be used with different applications to confirm their applicability in the centralized preprocessing approach. Data reduction/compression and time synchronization preprocessing procedures were presented but not evaluated. In addition, computational load should be evaluated for different time scale applications and data sizes but they are all considered a future work. 


\section{Bibliography}

Aggrey, G. (2007). A Study of Intelligent Oil and Gas Fields' Real Time Optimisation and its Value Quantification. PhD, Heriot-Watt University.

Aggrey, G. H., D. R. Davies, et al. (2006). Data Richness and Reliability in Smart-Field Management - Is There Value? SPE Annual Technical Conference and Exhibition. San Antonio, Texas, USA, Society of Petroleum Engineers.

Aggrey, G. H., D. R. Davies, et al. (2007). A Novel Approach of Detecting Water Influx Time in Multi-Zone and MultilateralCompletions using Real-Time Downhole Pressure Data. SPE Middle East Oil and Gas Show and Conference. Kingdom of Bahrain, Society of Petroleum Engineers.

Ajayi, A. A., D. Mathieson, et al. (2005). An Innovative Way of Integrating Reliability of Intelligent Well Completion System with Reservoir Modelling. Offshore Europe. Aberdeen, United Kingdom, Society of Petroleum Engineers.

Al-Hadhrami, A. K., L. Elliot, et al. (2003). "A New Model for Viscous Dissipation in Porous Media Across a Range of Permeability Values." Transport in Porous Media 53: 6.

Al-Khalifah, A.-J. A., K. Aziz, et al. (1987). A New Approach to Multiphase Well Test Analysis. SPE Annual Technical Conference and Exhibition. Dallas, Texas, 1987 Copyright 1987, Society of Petroleum Engineers.

Alberts, G., S. Belfroid, et al. (2007). An Investigation Into the Need of a Dynamic Coupled Well-Reservoir Simulator. SPE Annual Technical Conference and Exhibition. Anaheim, California, U.S.A., Society of Petroleum Engineers.

Alves, I. N., F. J. S. Alhanati, et al. (1992). "A Unified Model for Predicting Flowing Temperature Distribution. in Wellbores and Pipelines." SPE Production Engineering 7(4): 363-367.

Athichanagorn, S., R. N. Horne, et al. (1999). Processing and Interpretation of Long-term Data from Permanent Downhole Pressure Gauges. SPE Annual Technical Conference and Exhibition. Houston, Texas, Society of Petroleum Engineers, Inc.

Bear, J. (1988). Dynamics of fluids in porous media, Courier Dover Publications.

Becker, S. R., E. J. Candès, et al. (2011). "Templates for convex cone problems with applications to sparse signal recovery." Mathematical Programming Computation 3(3): 165-218.

Birchenko, V. M., V. Demyanov, et al. (2008). Impact of Reservoir Uncertainty on Selection of Advanced Completion Type. SPE Annual Technical Conference and Exhibition. Denver, Colorado, USA, Society of Petroleum Engineers.

Bird, J. M. and N. Frost (1966). "Formation Productivity Evaluation From Temperature Logs." SPE Journal of Petroleum Technology 18(3): 301305.

Brezzi, F. and M. Fortin (1991). Mixed and Hydrid Finite Element Methods, Springer-Verlag Berlin and Heidelberg GmbH \& Co. K.

Brouwer, D. R., G. Nædal, et al. (2004). Improved Reservoir Management Through Optimal Control and Continuous Model Updating. SPE Annual 
Technical Conference and Exhibition. Houston, Texas, Society of Petroleum Engineers.

Candès, E. J., X. Li, et al. (2011). "Robust Principal Component Analysis?" Journal of the ACM 58 (3): 37.

Candès, E. J. and B. Recht (2009). "Exact Matrix Completion via Convex Optimization." Found. Comput. Math. 9(6): 717-772.

Candès, E. J., J. K. Romberg, et al. (2006). "Stable signal recovery from incomplete and inaccurate measurements." Communications on Pure and Applied Mathematics 59(8): 1207-1223.

Churchill, S. W. and H. H. S. Chu (1975). "Correlating equations for laminar and turbulent free convection from a horizontal cylinder." International Journal of Heat and Mass Transfer 18(9): 5.

Churchill, S. W. and H. H. S. Chu (1975). "Correlating equations for laminar and turbulent free convection from a vertical plate." International Journal of Heat and Mass Transfer 18(11): 6.

Coifman, R. R. and S. Lafon (2006). "Diffusion maps." Applied and Computational Harmonic Analysis: 5-30.

Culshaw, B. and A. Kersey (2008). "Fiber-Optic Sensing: A Historical Perspective." Journal of Lightwave Technology 26(9): 15.

Donoho, D. L. and J. M. Johnstone (1994). "Ideal spatial adaptation by wavelet shrinkage." Biometrika 81(3): 425-455.

Dropkin, D. and E. Somerscales (1965). "Heat Transfer by Natural Convection in Liquids Confined by two Parallel Plates Which are Inclined at Various Angles with Respect to the Horizon." Journal of Heat Transfer(87): 77-84.

Duru, O. O. and R. N. Horne (2010). "Modeling Reservoir Temperature Transients and Reservoir-Parameter Estimation Constrained to the Model." SPE Reservoir Evaluation \& Engineering 13(6).

Eck, J. (1999/2000). Downhole Monitoring: The Story So Far, Oilfield Review. Elhamifar, E. and R. Vidal (2009). Sparse subspace clustering. IEEE CVPR.

Elshahawi, H., M. S. Osman, et al. (1999). Use Of Temperature Data in Gas Well Tests. SPE Annual Technical Conference and Exhibition. Houston, Texas, Society of Petroleum Engineers.

Fan, J. and R. Li (2001). "Variable Selection via Nonconcave Penalized Likelihood and its Oracle Properties." Journal of the American Statistical Association 96(456): 1348-1360.

Friedemann, J. D., A. Varma, et al. (2008). Subsea Condition Monitoring: A Path to Increased Availability and Increased Recovery. Intelligent Energy Conference and Exhibition. Amsterdam, The Netherlands, Society of Petroleum Engineers.

Gambhir, H. S., A. Shrivastav, et al. (2008). Sensor Architecture for Open Hole Gravel Pack Completions. SPE Annual Technical Conference and Exhibition. Denver, Colorado, USA, Society of Petroleum Engineers.

Ghajar, A. J. and C. C. Tang (2010). Void Fraction and Flow Patterns of Twophase Gas-Liquid Flow in Various Pipe Inclinations. 7th International Conference on Heat Transfer, Fluid Mechanics, and Thermodynamics. Antalya, Turkey: 115-129.

Grebenkin, I. and D. R. Davies (2010). Analysis of the Impact of an Intelligent Well Completion on the Oil Production Uncertainty. SPE Russian Oil and Gas Conference and Exhibition. Moscow, Russia, Society of Petroleum Engineers. 
Grubert, M. A., J. Wan, et al. (2009). Coupled Completion and Reservoir Simulation Technology for Well Performance Optimization. SPE Annual Technical Conference and Exhibition. New Orleans, Louisiana, Society of Petroleum Engineers.

Gysling, D. L. and D. H. Loose (2003). SONAR-Based, Clamp-on Flowmeter for gas and liquid applications. ISA EXPO. Houston, USA. BI0036 Rev. B.

Hadley, M. and R. Kimish (2008). Distributed Temperature Sensor Measures Temperature Resolution in Real Time. SPE Annual Technical Conference and Exhibition. Denver, Colorado, USA, Society of Petroleum Engineers.

Hasan, A. R. and C. S. Kabir (2002). Fluid Flow and Heat Transfer In Wellbores, SPE.

Heaton, H. S., W. C. Reynolds, et al. (1964). "Heat Transfer in Annular Passages, Simultaneous Development of Velocity and Temperature Fields in Laminar Flow." International Journal of Heat and Mass Transfer 7: 19.

Hudson, J. D., I. N. Alves, et al. (2011). Formalization and Standardization of the Smart Well Valuation Workflow. SPE Annual Technical Conference and Exhibition. Denver, Colorado, USA, Society of Petroleum Engineers.

ISO/IEC (2007). ISO/IEC Guide 99:2007. International vocabulary of metrology - Basic and general concepts and associated terms (VIM), ISO/IEC.

ISO/IEC (2008). ISO/IEC Guide 98-3:2008. Uncertainty of measurement -- Part 3: Guide to the expression of uncertainty in measurement (GUM:1995), ISO/IEC.

Jaafar, M. Z., M. D. Jackson, et al. (2009). Measurements of Streaming Potential for Downhole Monitoring in Intelligent Wells. SPE Middle East Oil and Gas Show and Conference. Bahrain, Bahrain, Society of Petroleum Engineers.

Jackson, M. D., J. Vinogradov, et al. (2011). "Laboratory Measurements and Numerical Modeling of Streaming Potential for Downhole Monitoring in Intelligent Wells." SPE Journal(09).

Keller, Y., R. R. Coifman, et al. (2010). "Audio-Visual Group Recognition Using Diffusion Maps " IEEE Transactions on Signal Processing 58(1): 403-413.

Kelley, C. T. (2003). Solving Nonlinear Equations with Newton's Method, SIAM.

Kikani, J. and M. He (1998). Multi-resolution Analysis of Long-Term Pressure Transient Data Using Wavelet Methods. SPE Annual Technical Conference and Exhibition. New Orleans, Louisiana, 1998 Copyright 1998, Society of Petroleum Engineers Inc.

Kim, J.-y. and A. J. Ghajar (2006). "A general heat transfer correlation for nonboiling gas-liquid flow with different flow patterns in horizontal pipes." International Journal of Multiphase Flow 32(4): 447-465.

Kiryushkina, T., A. S. Sikandar, et al. (2011). Implementation of Customized Algorithms Extends Downhole Flowmeter Application in Two-Phase Oil/Gas Flow: A Subsea North Sea Case Study. Brasil Offshore. Maca $\cong$, Brazil, Society of Petroleum Engineers.

Kluth, E. L. E., M. P. Varnham, et al. (2000). Advanced Sensor Infrastructure for Real Time Reservoir Monitoring. SPE European Petroleum Conference. Paris, France, Copyright 2000, Society of Petroleum Engineers Inc.

Knudsen, S., G. B. Havsgard, et al. (2003). Permanently Installed High Resolution Fiber-Optic 3C/4D Seismic Sensor Systems for In-Well Imaging and Monitoring Applications. Sixth Pacific Northwest Fiber Optic Sensor Workshop, SPIE. 
Konopczynski, M. and A. Ajayi (2004). Design of Intelligent Well Downhole Valves for Adjustable Flow Control. SPE Annual Technical Conference and Exhibition. Houston, Texas, Society of Petroleum Engineers.

Lafon, S. (2004). Diffusion maps and geometric harmonics. Ph.D., Yale University.

Lau, H.-C. (2008). Good Practices in Progressing a Smart Well Portfolio. International Petroleum Technology Conference. Kuala Lumpur, Malaysia, International Petroleum Technology Conference.

Leskens, M., J. P. M. Smeulers, et al. (2008). Downhole Multiphase Metering In Wells By Means Of Soft-sensing. Intelligent Energy Conference and Exhibition. Amsterdam, The Netherlands, Society of Petroleum Engineers.

Lin, Z., M. Chen, et al. (2010). The Augmented Lagrange Multiplier Method for Exact Recovery of Corrupted Low-Rank Matrices, arXiv:1009.5055v2 [math.OC].

Mathieson, D., J. Rogers, et al. (2003). Reliability assurance, managing the growth of Intelligent Completion technology. SPE Annual Technical Conference and Exhibition. Denver, Colorado, Society of Petroleum Engineers.

Mathis, W. and G. Thonhouser (2007). Mastering Real-Time Data Quality Control - How to Measure and Manage the Quality of (Rig) Sensor Data. 11th International Conference on Petroleum Data Integration, Information and Data Management. Amsterdam.

McKinley, R. M. and F. M. Bower (1979). "Specialized Applications of Noise Logging." SPE Journal of Petroleum Technology(11).

Mestayer, J., B. Cox, et al. (2011). "Field trials of distributed acoustic sensing for geophysical monitoring." SEG Technical Program Expanded Abstracts 30(1): 4253-4257.

Molenaar, M. M., D. Hill, et al. (2011). First Downhole Application of Distributed Acoustic Sensing (DAS) for Hydraulic Fracturing Monitoring and Diagnostics. SPE Hydraulic Fracturing Technology Conference. The Woodlands, Texas, USA, Society of Petroleum Engineers.

Mougenot, D., A. Cherepovskiy, et al. (2011). MEMS-based accelerometers: expectations and practical achievements. First Break 29.

Muradov, K. M. (2010). Temperature Modelling and Real-time Flow Rate Allocation in Wells with Advanced Completion. PhD, Heriot-Watt University.

Muradov, K. M. and D. R. Davies (2008). Prediction of Temperature Distribution in Intelligent Wells. SPE Russian Oil and Gas Technical Conference and Exhibition. Moscow, Russia, Society of Petroleum Engineers.

Muradov, K. M. and D. R. Davies (2009). Temperature Modelling and Analysis of Wells with Advanced Completion. EUROPEC/EAGE Conference and Exhibition. Amsterdam, The Netherlands, Society of Petroleum Engineers.

Muradov, K. M. and D. R. Davies (2010). Novel Analytical Methods of Temperature Interpretation in Horizontal Wells. SPE EUROPEC/EAGE Annual Conference and Exhibition. Barcelona, Spain, Society of Petroleum Engineers.

Muradov, K. M. and D. R. Davies (2011). "Novel Analytical Methods of Temperature Interpretation in Horizontal Wells." SPE Journal(09). 
Muradov, K. M. and D. R. Davies (2012). Temperature Transient Analysis in a Horizontal, Multi-zone, Intelligent Well. SPE Intelligent Energy International. Utrecht, The Netherlands.

Naevdal, G., E. Vefring, et al. (2001). "A New Methodology for the Optimization of the Placement of Downhole Production-Monitoring Sensors." SPE Journal(03).

Nyhavn, F., F. Vassenden, et al. (2000). Reservoir Drainage with Downhole Permanent Monitoring and Control Systems. Real-Time Integration of Dynamic Reservoir Performance Data and Static Reservoir Model Improves Control Decisions. SPE Annual Technical Conference and Exhibition. Dallas, Texas, 2000,. Society of Petroleum Engineers Inc.

Oliveira, J. L. G., J. C. Passos, et al. (2009). "Mass flow rate measurements in gas-liquid flows by means of a venturi or orifice plate coupled to a void fraction sensor." Experimental Thermal and Fluid Science 33(2): 253-260.

Olsen, S. (2011). On Automatic Data Processing and Well-Test Analysis in Realtime Reservoir Management Applications. PhD, University of Bergen.

Olsen, S. and J.-E. Nordtvedt (2006). Experience From the Use of Automatic Well-Test Analysis. SPE Annual Technical Conference and Exhibition. San Antonio, Texas, USA, Society of Petroleum Engineers.

Ong, J. T., M. Aymond, et al. (2007). Inverted Venturi: Optimising Recovery Through Flow Measurement. SPE Annual Technical Conference and Exhibition. Anaheim, California, U.S.A., Society of Petroleum Engineers.

Ouyang, L.-B. and K. Aziz (1998). A Simplified Approach to Couple Wellbore Flow and Reservoir Inflow for Arbitrary Well Configurations. SPE Annual Technical Conference and Exhibition. New Orleans, Louisiana, 1998 Copyright 1998, Society of Petroleum Engineers Inc.

Pari, M. N., A. H. Kabir, et al. (2009). Smart well- Benefits, Types of Sensors, Challenges, Economic Consideration, and Application in Fractured Reservoir. SPE Saudi Arabia Section Technical Symposium. AlKhobar, Saudi Arabia, Society of Petroleum Engineers.

Pico, C., R. Aguiar, et al. (2009). Wavelet Filtering of Permanent Downhole Gauge Data. Latin American and Caribbean Petroleum Engineering Conference. Cartagena de Indias, Colombia, Society of Petroleum Engineers.

Rambow, F. H. K., D. E. Dria, et al. (2010). "Real-Time Fiber-Optic Casing Imager." SPE Journal(12).

Ramey Jr., H. J. (1962). "Wellbore Heat Transmission." SPE Journal of Petroleum Technology 14(4): 427-435.

Rausand, M. and A. Høyland (2004). System Reliability Theory: Models, Statistical methods, and Applications, Wiley.

Recht, B., M. Fazel, et al. (2010). "Guaranteed Minimum-Rank Solutions of Linear Matrix Equations via Nuclear Norm Minimization." Siam Review 52(3): 471-501.

Ribeiro, P. M., A. P. Pires, et al. (2008). "Use of Wavelet Transform in PressureData Treatment." SPE Production \& Operations 23(1): pp. 24-31.

Sieder, E. N. and G. E. Tate (1936). "Heat Transfer and Pressure Drop of Liquids in Tubes." Industrial \& Engineering Chemistry 28(12): 7.

Silva, M. F. D., K. M. Muradov, et al. (2012). Modeling and Analysis of Temperature Transients Caused by Step-Like Change of Downhole Flow Control Device Flow Area. SPE Latin America and Caribbean Petroleum 
Engineering Conference. Mexico City, Mexico, Society of Petroleum Engineers.

Silva, M. F. D., K. M. Muradov, et al. (2012). Review, Analysis and Comparison of Intelligent Well Monitoring Systems. SPE Intelligent Energy International. Utrecht, The Netherlands, Society of Petroleum Engineers.

Stephan, K. (1962). "Heat transfer in turbulent and laminar flow in annular slits." Chem. Ing. Tech. 34: 207-212.

Strack, K. (2010). Advances in Electromagnetics for Reservoir Monitoring. 8th International Conference \& Exposition on Petroleum Geophysics, Hyderabad.

Sui, W., C. A. Ehlig-Economides, et al. (2010). Determining Multilayer Formation Properties from Transient Temperature and Pressure Measurements in Comingled Gas Wells. International Oil and Gas Conference and Exhibition in China. Beijing, China, Society of Petroleum Engineers.

Sui, W., D. Zhu, et al. (2008). Model for Transient Temperature and Pressure Behavior in Commingled Vertical Wells. SPE Russian Oil and Gas Technical Conference and Exhibition. Moscow, Russia, Society of Petroleum Engineers.

Unalmis, O. H., E. S. Johansen, et al. (2010). Evolution in Optical Downhole Multiphase Flow Measurement: Experience Translates into Enhanced Design. SPE Intelligent Energy Conference and Exhibition. Utrecht, The Netherlands, Society of Petroleum Engineers.

Valiullin, R. A., A. Ramazanov, et al. (2009). Temperature Logging in Russia: Development History of Theory, Technology of Measurements and Interpretation Techniques. Kuwait International Petroleum Conference and Exhibition. Kuwait City, Kuwait, Society of Petroleum Engineers.

Vicente, R., C. Sarica, et al. (2001). A Two-Phase Model Coupling Reservoir and Horizontal Well Flow Dynamics. SPE Latin American and Caribbean Petroleum Engineering Conference. Buenos Aires, Argentina, Copyright 2001, Society of Petroleum Engineers Inc.

Wilson, S., G. L. Floch, et al. (2008). Emerging Geophysical Technologies: Downhole Microseismic Reservoir Monitoring (MRM): A New Instrumentation Technology for Monitoring the Digital Oilfield.

Yoshioka, K., D. Zhu, et al. (2005). A Comprehensive Model of Temperature Behavior in a Horizontal Well. SPE Annual Technical Conference and Exhibition. Dallas, Texas, Society of Petroleum Engineers.

Zhang, H. J., W. T. Yue, et al. (2005). "Investigation of oil-air two-phase mass flow rate measurement using venturi and void fraction sensor." Journal of Zhejiang University Science 6A(6): 601-606.

Zhou, Q., D. Julander, et al. (2002). Experiences With Casedhole Resistivity Logging For Reservoir Monitoring. SPWLA 43rd Annual Logging Symposium, Society of Petrophysicists \& Well Log Analysts.

Zhou, Z., X. Li, et al. (2010). Stable Principal Component Pursuit, arXiv:1001.2363v1 [cs.IT].

Zisk, E. J. (2005). Optical In-Well Permanent Monitoring-Initial Promise Now A Reality?

Zuber, N. and J. Findley (1965). "Average Volumetric Concentration in TwoPhase Systems." Journal of Heat Transfer 87. 


\section{Appendix A}

\section{Fanning Friction Factor}

Fanning friction factor is a dimensionless number that represents a dimensionless shear stress at the pipe wall (Figure A.1) and it represents onefourth of the Darcy friction factor:

$$
f_{F}=\frac{\tau_{\text {wall }}}{\frac{1}{2} \rho u^{2}}
$$

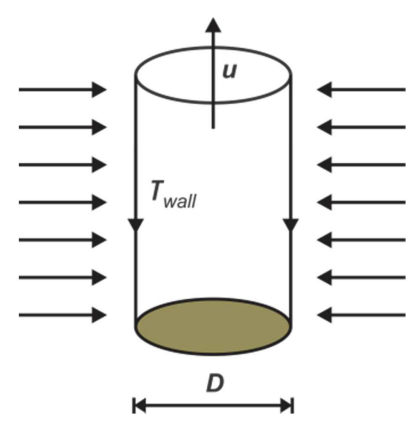

Figure A.1 - Shear stress at the pipe wall.

Various friction factors correlations are available but Chen correlation is used as recommended by Hasan and Kabir (Hasan and Kabir 2002). Even the friction factor effect is not a dominant effect for vertical wells it is considered to take into account for high flow rate wells.

Simplified multiphase flow models use the same single phase approach averaging the phases over a volume. Dispersed and continuous phases are combined and modeled as a new continuous mixture phase. Hence, the results from single phase can be applied:

Laminar flow:

$\mathrm{f}_{\mathrm{F}}=\frac{16}{\mathrm{R}_{\mathrm{m}}}$ $\mathrm{R}_{\mathrm{m}} \leq 2300$

Transitional flow:

$$
\mathrm{f}_{\mathrm{F}}=\mathrm{f}_{\mathrm{FL}}+\frac{\left(\mathrm{R}_{\mathrm{m}}-\mathrm{R}_{\mathrm{mL}}\right)}{\left(\mathrm{R}_{\mathrm{mT}}-\mathrm{R}_{\mathrm{mL}}\right)}\left(\mathrm{f}_{\mathrm{FT}}-\mathrm{f}_{\mathrm{FL}}\right) \quad 2300<\mathrm{R}_{\mathrm{m}}<4000
$$

Turbulent flow (Chen correlation):

$$
\frac{1}{\sqrt{\mathrm{f}_{\mathrm{F}}}}=-4 \log \left[\frac{\varepsilon}{3.7065 \mathrm{D}}-\frac{5.0452}{\mathrm{R}_{\mathrm{m}}} \log \left(\frac{\varepsilon^{1.1098}}{2.8257}+\frac{5.8506}{\mathrm{R}_{\mathrm{m}}^{0.8981}}\right)\right] \quad \mathrm{R}_{\mathrm{m}} \geq 4000
$$


Where:

$$
\begin{aligned}
& \mathrm{R}_{\mathrm{m}}=\frac{\rho_{\mathrm{m}} \mathrm{u}_{\mathrm{m}} \mathrm{D}}{\mu_{\mathrm{m}}} \\
& \mu_{\mathrm{m}}=\sum_{\mathrm{j}} \mu_{\mathrm{j}} \mathrm{y}_{\mathrm{j}}
\end{aligned}
$$

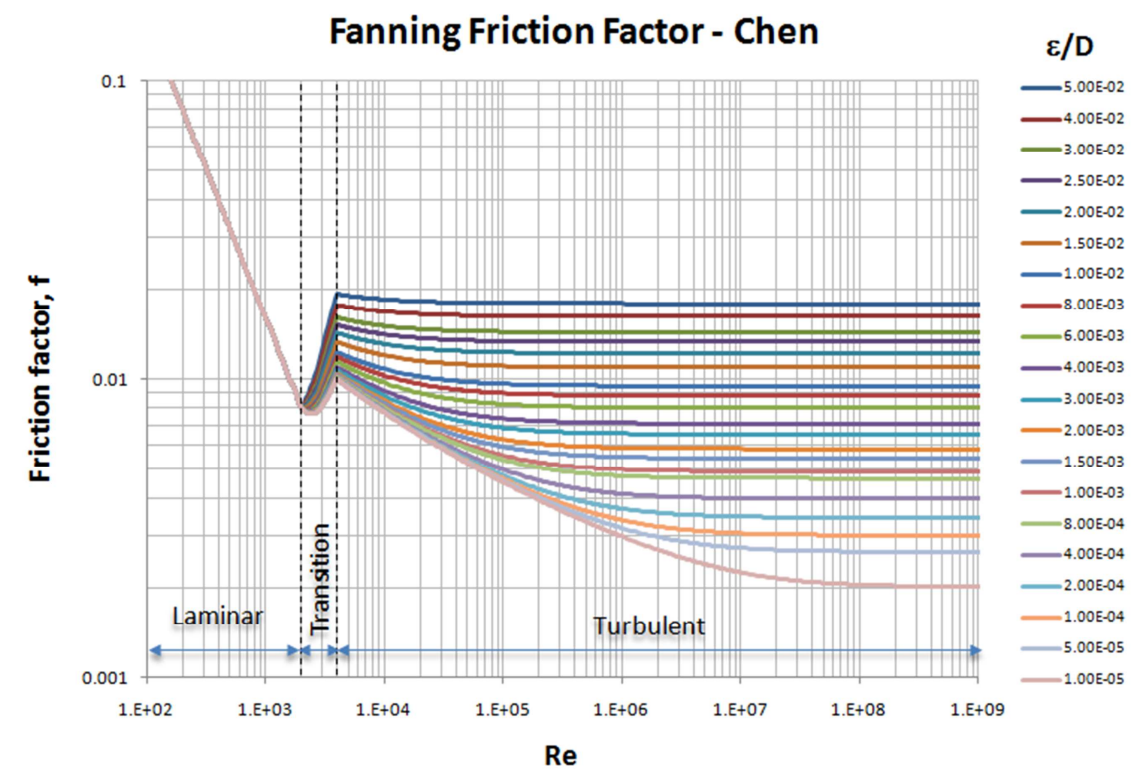

Figure A.2 - Chen (1979) - Fanning friction factor.

It should be noted that for the annulus the hydraulic diameter is used to calculate Reynolds number:

$$
\mathrm{D}_{\mathrm{h}}=\frac{4 \text { cross sectional area }}{\text { wetted perimeter }}
$$

Correction factor due to influx in production wells (porous pipe) is also applied (Ouyang and Aziz 1998):

Laminar flow:

$$
\mathrm{f}_{\mathrm{F}}=\frac{16}{\mathrm{R}_{\mathrm{m}}}\left(1+0,04304 \mathrm{R}_{\mathrm{e}}^{0.6142}\right)
$$

Turbulent flow:

$$
\mathrm{f}_{\mathrm{F}}=\mathrm{f}_{\mathrm{F} 0}\left(1-0.0153 \mathrm{R}_{\mathrm{e}}^{0.3978}\right)
$$

Where:

$\mathrm{R}_{\mathrm{e}}=\frac{\rho_{\mathrm{I}} \mathrm{u}_{\mathrm{I}} \mathrm{D}}{\mu_{\mathrm{I}}}$

I - Index for radial inflow mixture. 


\section{Appendix B}

\section{Drift-flux model}

The drift-flux multiphase model was introduced by Zuber and Findlay for two-phase flow in 1965 (Zuber and Findley 1965). For a gas-liquid flow in a vertical pipe the superficial gas velocity (cross sectional area averaged velocity) is expressed as a sum of the distribution coefficient $\left(C_{o}\right)$ and the gas drift velocity $\left(u_{g m}\right)$. It captures not only the mechanisms of higher concentration of gas near the center of the pipe $\left(C_{o} u_{m}\right)$ but also the tendency of buoyancy $\left(u_{\mathrm{gm}}\right)$. This explains why gas inside a vertical pipe moves faster than liquid:

$$
u_{g}=C_{o} u_{m}+u_{g m}
$$

The average mixture velocity is defined as the sum of the superficial velocities:

$$
\begin{aligned}
& u_{g}=\frac{u_{s g}}{x_{g}} \\
& u_{l}=\frac{u_{s l}}{1-x_{g}}
\end{aligned}
$$

Where:

$$
x_{g}=\frac{A_{g}}{A_{g}+A_{l}}-\text { gas void fraction }
$$

Drift-flux model is used in this thesis through correlations (Ghajar and Tang 2010):

$$
\begin{aligned}
& x_{g}=\frac{u_{s g}}{C_{o}\left(u_{s g}+u_{s l}\right)+u_{g m}} \\
& C_{o}=\frac{u_{s g}}{u_{s g}+u_{s l}}\left(1+\left(\frac{u_{s l}}{u_{s g}}\right)^{\left(\frac{\rho_{g}}{\rho_{l}}\right)^{0.1}}\right) \\
& u_{g m}=2.9(1.22+1.22 \sin \theta)^{\frac{p_{a t m}}{p_{s j s}}}\left(g D \sigma(1+\cos \theta)\left(\rho_{l}-\rho_{g}\right) / \rho_{l}^{2}\right)^{0.25}
\end{aligned}
$$

Where:

$\sigma$ - Superficial tension;

$\theta-$ Angle with vertical axis. 
A general two-phase heat transfer correlation (Kim and Ghajar 2006) for estimating the heat transfer coefficients regardless of flow pattern, gas-liquid combination and pipe inclination angle is also used. It is valid for liquid and permanent gas without taking phase changing into account.

The general heat transfer correlation is described by:

$$
h_{t p}=F_{p} h_{l}\left(1+0.55\left(\frac{\chi}{1-\chi}\right)^{0.1}\left(\frac{1-F_{p}}{F_{p}}\right)^{0.4}\left(\frac{P r_{g}}{P r_{l}}\right)^{0.25}\left(\frac{\mu_{l}}{\mu_{g}}\right)^{0.25}\left(I^{*}\right)^{0.25}\right)
$$

Where:

Gas quality $(\chi)$ :

$$
\begin{gathered}
\chi=\frac{\dot{m}_{g}}{\dot{m}_{g}-\dot{m}_{l}} \\
\dot{m}_{g}=\rho_{g} u_{s g} A \\
\dot{m}_{l}=\rho_{l} u_{s l} A
\end{gathered}
$$

Flow pattern factor $\left(F_{p}\right)$ and inclination factor $\left(I^{*}\right)$ :

$$
\begin{aligned}
& F_{p}=\left(1-x_{g}\right)+x_{g}\left(\frac{2}{\pi} \tan ^{-1}\left(\sqrt{\frac{\rho_{g}\left(u_{g}-u_{l}\right)^{2}}{g D\left(\rho_{l}-\rho_{g}\right)}}\right)\right)^{2} \\
& I^{*}=1+\frac{g D^{2}\left(\rho_{l}-\rho_{g}\right)}{\sigma}|\sin \theta|
\end{aligned}
$$

Liquid phase heat transfer correlation (Sieder and Tate 1936):

$$
\begin{aligned}
& h_{l}=\frac{0.027 \operatorname{Re}_{l}^{0.8} \operatorname{Pr}_{l}^{1 / 3} K_{l}}{D} \\
& \operatorname{Re}_{l}=\frac{\rho_{l} u_{l} D}{\mu_{l}\left(1-x_{g}\right)^{0.5}}
\end{aligned}
$$

In the heat transfer coefficient of the liquid phase the correction due to the viscosity term was considered negligible. The 1D model used to describe the behavior of the annulus and tubing cannot capture this effect because it is averaged within $T\left(r=r_{w}\right)$ to $T(r=0)$ and therefore it is meaningless.

The drift-flux multiphase model is used for application where there is gas/liquid and it is used only in the well segment (annulus and tubing). 


\section{Appendix C}

\section{Heat Transfer Coefficients}

A reservoir layer/well segment is shown in Figure C.1 with its radial layers: tubing, annulus, cement and formation. The heat transfer mechanisms among them can be described by conduction, convection and radiation (neglected here). The overall heat transfer coefficients used in the temperature model is taken into account the individual conductive and convective barriers to transfer heat within the wellbore. Heat transfer coefficients were used in the development of this thesis assuming that well transient is faster than reservoir transient therefore it is possible to reach thermal equilibrium allowing heat transfer coefficient to be defined.

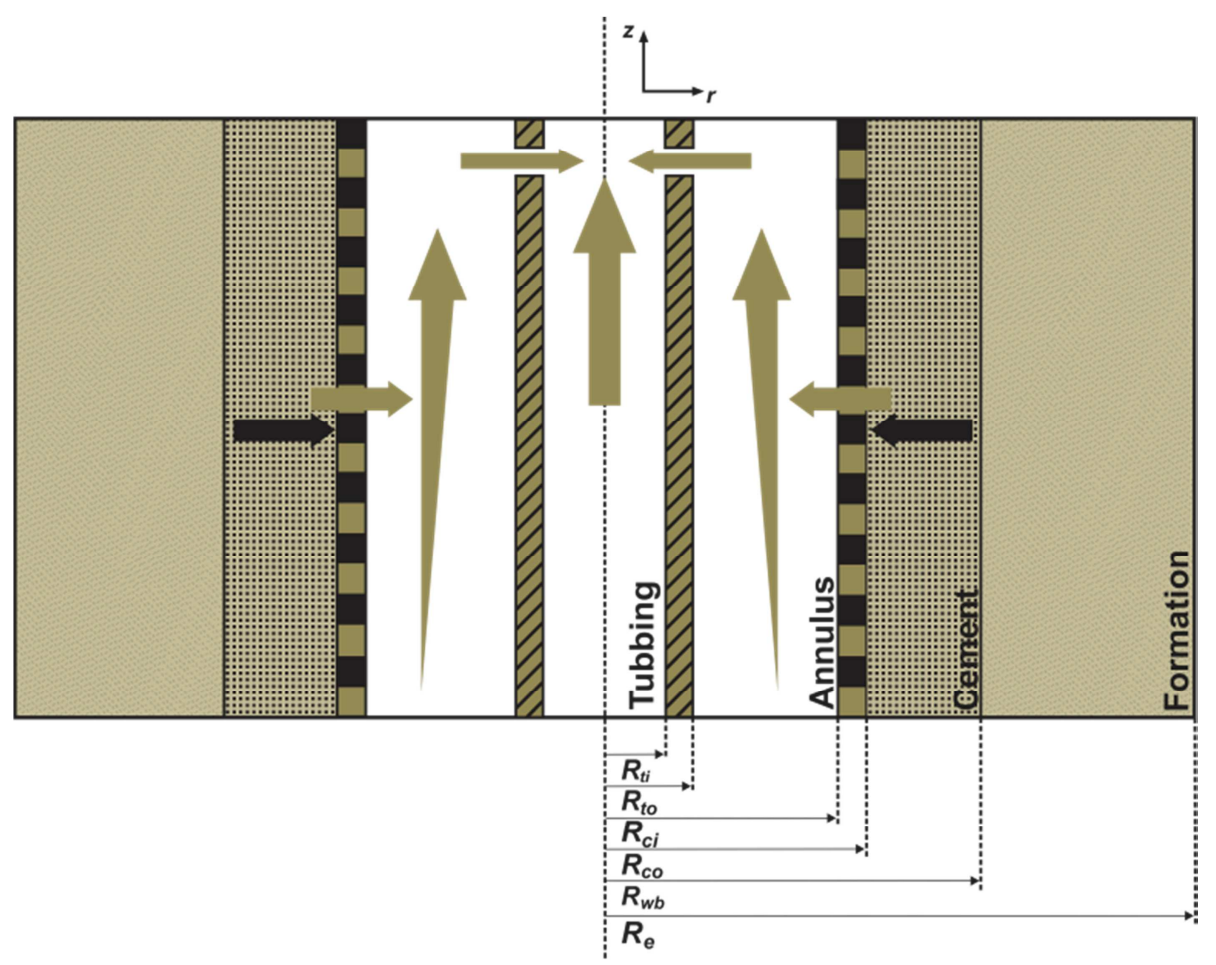

Figure C.1 - Heat transfer within wellbore.

\section{$h_{t}$ - Heat transfer coefficient between tubing to annulus}

The convection and conduction due to the fluids in the annulus and tubing as well as the conduction through the tubing wall are taken into account:

$$
U_{t a}=\frac{1}{\frac{1}{h_{t}}+\frac{R_{t i}}{K_{t}} \ln \left(\frac{R_{t o}}{R_{t i}}\right)+\frac{R_{t i}}{R_{t o} h_{a}}}
$$


The convection coefficients $h_{t}$ and $h_{a}$ are based in correlations. The appropriate coefficient for the annulus is chosen based on Reynolds number. The tubing and annulus can be experiencing stagnant, laminar, and transitional or turbulent flow. We use the fact that superposition is valid for laminar flow with constant heat flux and simultaneously developing velocity and temperature profiles (Heaton, Reynolds et al. 1964).

Forced convection $(R e>2300)$ :

Turbulent or transitional flow (Sieder and Tate 1936):

For tubing:

$$
h_{t}=\frac{0.027 \operatorname{Re}_{t}^{0.8} \operatorname{Pr}_{t}^{1 / 3} K_{t}}{2 R_{t i}}
$$

For annulus:

$$
h_{a}=\frac{0.027 \operatorname{Re}_{a}^{0.8} \operatorname{Pr}_{a}^{1 / 3} K_{a}}{2\left(R_{c i}-R_{t o}\right)}
$$

Natural convection $(R e<2300)$ :

Inner wall (Sieder and Tate 1936):

For tubing:

$$
h_{t}=1.84 \frac{2 R_{t i}}{K_{t}}\left(R a_{t}\right)^{1 / 3} \text { or } h_{t}=3.66 \text { for } 2 R_{t i} \square L
$$

For annulus:

In this case we use superposition (Heaton, Reynolds et al. 1964) and divide the problem in two:

- A pipe experiencing inner convection with outer insulated:

$$
h_{\text {ainner }}=1.84 \frac{2\left(R_{c i}-R_{t o}\right)}{K_{a}}\left(R a_{a}\right)^{1 / 3} \text { or } h_{a_{\text {inner }}}=3.66 \text { for } 2\left(R_{c i}-R_{t o}\right) \square L
$$

Where

- $\mathrm{L}$ is the length of the well segment.

- A pipe experiencing outer convection with inner insulated. In this case the orientation is important:

Vertical outer wall (Churchill and Chu 1975): 


$$
h_{\text {aouter } 1}=\left(0.825+\frac{0.387 R a_{a}^{1 / 6}}{\left(1+\left(\frac{0.492}{\operatorname{Pr}_{a}}\right)^{9 / 16}\right)^{8 / 27}}\right)^{2} \frac{2\left(R_{c i}-R_{\text {to }}\right)}{K_{a}}
$$

It is used for inclinations of $45^{\circ}$ and above.

Horizontal outer wall (Churchill and Chu 1975):

$$
h_{\text {aouter } 2}=\left(0.6+\frac{0.387 R a_{a}^{1 / 6}}{\left(1+\left(\frac{0.559}{\operatorname{Pr}_{a}}\right)^{9 / 16}\right)^{8 / 27}}\right)^{2} \frac{2\left(R_{c i}-R_{t o}\right)}{K_{a}}
$$

It is used for inclinations of $45^{\circ}$ and below.

Therefore, $h_{\mathrm{a}}$ is:

$h_{a}=h_{\text {ainner }}+h_{\text {aouter }}$

Stagnant fluid or pure natural convection (Dropkin and Somerscales 1965):

In this case we assume that we have stagnant fluid only in the annulus, hence:

$$
h_{a}=\frac{0.049 R a_{a}^{1 / 3} \operatorname{Pr}_{a}^{0.074} K_{a}}{R_{t o} \ln \left(\frac{R_{c i}}{R_{t o}}\right)}
$$

It should be noted that strong buoyancy effect is expected in the flow which $\frac{G r}{\mathrm{Re}^{2}}$ is close to unity, otherwise it may be ignored. In pure natural convection, the strength of the buoyancy-induced flow is measured by the $R a$ where less than $10^{8}$ is laminar flow and above $10^{10}$ is turbulent flow.

The heat transfer coefficients $h_{t}$ and $h_{a}$ have to take into account the simultaneous flow inside the tubing and inside the annulus. Stephan correlation (Stephan 1962) is used for this purpose:

$$
h^{\prime}=h \frac{\left(R_{c i}-R_{t o}\right)}{R_{t i}}\left(\frac{0.86\left(\frac{R_{t i}}{R_{c i}}\right)^{0.84}+\left(1-0.14\left(\frac{R_{t i}}{R_{c i}}\right)^{0.6}\right)}{1+\frac{R_{t i}}{R_{c i}}}\right)
$$


In the above equations the dimensionless terms were described as:

Prandtl Number:

$$
\operatorname{Pr}=\frac{C_{p} \mu}{K}
$$

Grashof Number:

$$
\begin{aligned}
G r_{a} & =\frac{\left(R_{c i}-R_{t o}\right)^{3} g \rho_{a}^{2} \beta_{a} \Delta T_{a}}{\mu_{a}^{2}} \\
G r_{t} & =\frac{\left(R_{t i}\right)^{3} g \rho_{t}^{2} \beta_{t} \Delta T_{t}}{\mu_{t}^{2}}
\end{aligned}
$$

Raleigh Number:

$$
R a=G r P r
$$

Reynolds Number:

$$
\operatorname{Re}_{a}=\frac{\rho_{a} u_{a} 2\left(R_{c i}-R_{t o}\right)}{\mu_{a}}
$$

$$
\operatorname{Re}_{t}=\frac{\rho_{t} u_{t} 2 R_{t i}}{\mu_{a}}
$$

\section{$h_{a}$ - Heat transfer coefficient between annulus to formation}

It takes into account the conduction through the casing wall and cement as well as the convection and conduction through the formation.

$$
U_{a f}=\frac{1}{\frac{1}{h_{a}}+\frac{1}{U_{a w b}}}
$$

- Heat transfer between annulus and wellbore

It takes into account the conduction through the casing wall and cement.

$$
U_{a w b}=\frac{1}{\frac{R_{c i}}{K_{c a \sin g}} \ln \left(\frac{R_{c o}}{R_{c i}}\right)+\frac{R_{c i}}{K_{c e m e n t}} \ln \left(\frac{R_{w b}}{R_{c o}}\right)}
$$




\section{Appendix D}

\section{Cylindrical-Shape Reservoir Layer Equations}

Two phase formulation for an adiabatic cylindrical-shaped reservoir layer (Figure D.1) experiencing transient flow is derived in this appendix.

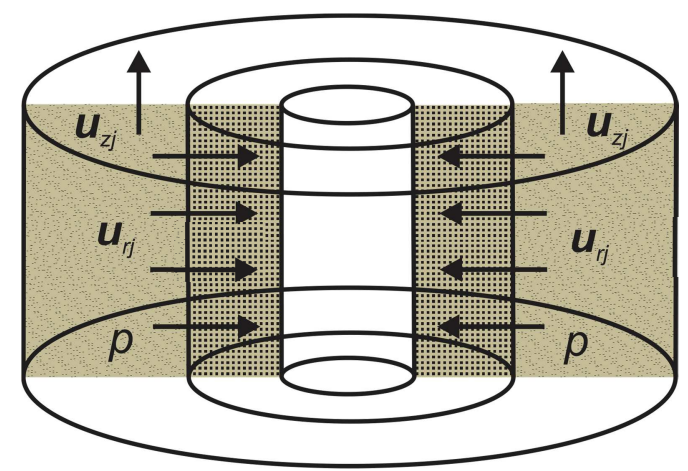

Figure D.1 - Cylindrical-shaped reservoir.

The following group of assumptions was used for the mass balance and flow equations for the control volume shown in Figure D.2:

- Rock and fluids are slightly compressible;

- Porous media is considered homogeneous;

- Capillarity pressure can be neglected $\left(p_{w}=p_{o}=p\right)$;

- Fluid saturations are constant during the simulation period;

- Total compressibility times pressure is much smaller than one $\left(c_{t} p \square 1\right)$;

- Immiscible fluids;

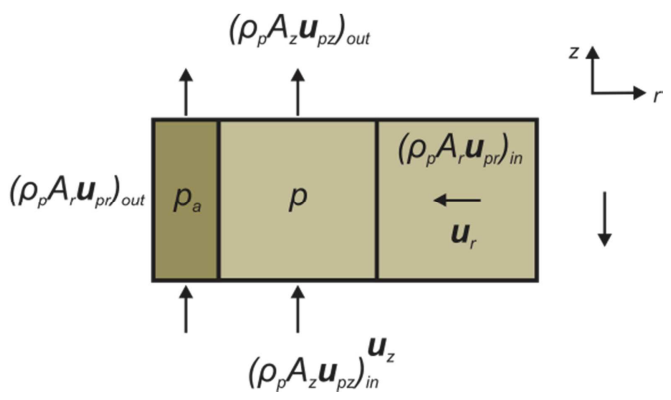

Figure D.2 - Control volume of the reservoir mass balance and flow equations. 
Mass Balance:

$$
\begin{aligned}
& \frac{\partial\left(\phi \rho_{p} S_{p}\right)}{\partial t}+\nabla \cdot\left(\rho_{p} \boldsymbol{u}_{p}\right)=0 \\
& \rho_{p} S_{p} \frac{\partial \phi}{\partial t}+\phi \frac{\partial\left(\rho_{p} S_{p}\right)}{\partial t}=-\rho_{p} \nabla \cdot \boldsymbol{u}_{p}-\boldsymbol{u}_{p} \cdot \nabla \rho_{p}
\end{aligned}
$$

Defining formation and fluids compressibility as:

$c_{f}=\frac{1}{\phi} \frac{\partial \phi}{\partial p}$ and $c_{p}=\frac{1}{\rho_{p}} \frac{\partial \rho_{p}}{\partial p}$

Making the necessary arrangements yields:

$\phi c_{t} \frac{\partial p}{\partial t}=-\nabla \cdot \boldsymbol{u}-\sum_{j} c_{j} \boldsymbol{u}_{j} \cdot \nabla p$

Where:

$c_{t}=c_{f}+\sum_{j} c_{j} S_{j}$

$\boldsymbol{u}=\sum_{j} \boldsymbol{u}_{j}$

\section{Darcy's Equation:}

$$
\boldsymbol{u}_{p}=-\frac{k_{r p}}{\mu_{p}} \boldsymbol{k} \cdot\left(\nabla \mathrm{p}-\rho_{p} \mathrm{~g} \nabla z\right)
$$

Defining relative mobility as:

$$
\lambda_{p}=\frac{k_{r p}}{\mu_{p}}
$$

Making the substitution in Darcy's equation:

$$
\boldsymbol{u}_{p}=-\lambda_{p} \boldsymbol{k} \cdot\left(\nabla \mathrm{p}-\rho_{p} \mathrm{~g} \nabla z\right)
$$

Combining with mass balance equation yields:

$$
\phi c_{t} \frac{\partial p}{\partial t}=\nabla \cdot\left(\sum_{j} \lambda_{j} \boldsymbol{k} \cdot\left(\nabla \mathrm{p}-\rho_{j} \mathrm{~g} \nabla z\right)\right)+\sum_{j} c_{j}\left(\nabla \mathrm{p}-\rho_{j} \mathrm{~g} \nabla z\right) \cdot \nabla p
$$

Making the assumption that reservoir layer height and $(\nabla p)^{2}$ are small implies hence the term $\sum_{j} c_{j}\left(\nabla \mathrm{p}-\rho_{j} \mathrm{~g} \nabla z\right) \cdot \nabla p$ can be dropped resulting in:

$$
\phi c_{t} \frac{\partial p}{\partial t}=\nabla \cdot\left(\lambda_{t} \boldsymbol{k} \cdot\left(\nabla p-\rho_{t} \mathrm{~g} \nabla z\right)\right)
$$

Where: 


$$
\begin{aligned}
& \lambda_{t}=\sum_{j} \lambda_{j} \\
& \rho_{t}=\left(\sum_{j} \rho_{j} \frac{\lambda_{j}}{\lambda_{t}}\right)
\end{aligned}
$$

It should be noted that the equation derived has the same mathematical form of the classic diffusivity equation from the well test analysis.

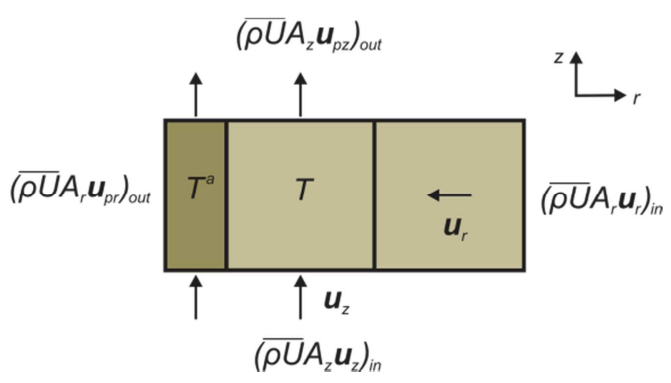

Figure D.3 - Volume control of the reservoir energy balance.

\section{Energy balance}

According to Bear (Bear 1988), the temperature behavior of the fluids in porous media is based on the basic mass and heat transfer principles. Therefore, it can be derived by the general energy balance equation (Bird and Frost 1966):

$\frac{\partial \overline{\rho U}}{\partial t}+\nabla \cdot(\overline{\rho U} \boldsymbol{u})=-p \nabla \cdot \boldsymbol{u}-\nabla \cdot \boldsymbol{q}_{T}-\boldsymbol{\tau}: \nabla \boldsymbol{u}$

Enthalpy definition:

$$
H=U+\frac{p}{\rho}
$$

Fourier law:

$$
\boldsymbol{q}_{T}=-\nabla \cdot\left(\boldsymbol{k}_{T} \nabla T\right)
$$

Total conductivity (formation and fluids):

$\boldsymbol{k}_{T}=\phi \boldsymbol{k}_{s}+(1-\phi) \boldsymbol{k}_{f}$

Bulk internal energy (formation and fluids):

$\overline{\rho U}=\phi \rho_{s} U_{s}+(1-\phi) \rho_{f} U_{f}$

According to Al-Hadhrami (Al-Hadhrami, Elliot et al. 2003) the term $-\boldsymbol{\tau}: \nabla \boldsymbol{u}$ can be replaced by $\boldsymbol{u} \cdot \nabla \mathrm{p}$, resulting:

$$
\frac{\partial}{\partial t}\left(\phi \rho H-\phi p+(1-\phi) \rho_{f} U_{f}\right)=-\nabla \cdot(\rho H \boldsymbol{u})+\nabla \cdot\left(\boldsymbol{k}_{T} \nabla \mathrm{T}\right)
$$


Assumptions:

The control volume (Figure D.3) is in local thermal equilibrium (quasisteady-state assumption);

Rock density is constant:

$d U_{f} \approx d H_{f}=C_{p f} d T_{f}$

The following approximation is valid:

$d H=C_{p} d T+\frac{1}{\rho}(1-\beta T) d p$

Defining:

$\overline{\rho C_{p}}=\phi \rho C_{p}+(1-\phi) \rho_{f} C_{p f}$

Making the assumptions and final arrangements, yields:

$\overline{\rho C_{p}} \frac{\partial T}{\partial t}-\phi \beta T \frac{\partial p}{\partial t}-\phi c_{f}\left(p+\rho_{f} C_{p f} T\right) \frac{\partial p}{\partial t}=\rho \boldsymbol{u} C_{p} \nabla \mathrm{T}+(1-\beta T) \boldsymbol{u} \cdot \nabla p+\nabla \cdot\left(\boldsymbol{k}_{T} \nabla \mathrm{T}\right)$

The multiphase formulation can be derived using the same approach resulting:

$$
\begin{aligned}
& \left(\phi \sum_{j} \rho_{j} S_{j} C_{p j}+(1-\phi) \rho_{f} C_{p f}\right) \frac{\partial T}{\partial t}-\phi\left(\sum_{j} S_{j} \beta_{j}\right) T \frac{\partial p}{\partial t}-\phi c_{f}\left(p+\rho_{f} C_{p f} T\right) \frac{\partial p}{\partial t}= \\
& \left(\sum_{j} \rho_{j} S_{j} C_{p j}\right) \boldsymbol{u} \cdot \nabla \mathrm{T}+\left(1-\left(\sum_{j} \beta_{j}\right) T\right) \boldsymbol{u} \cdot \nabla p+\nabla \cdot\left(\left(\phi \sum_{j} S_{j} \boldsymbol{k}_{j}+(1-\phi) \boldsymbol{k}_{f}\right) \nabla \mathrm{T}\right)
\end{aligned}
$$

The meaning of the equation terms can be summarized as:

LHS:

Term 1 - transient temperature variation;

Term 2 - transient formation and fluid expansion or compression;

RHS:

Term 3 - heat convection;

Term 4 - viscous dissipation and fluid expansion or compression;

Term 5 - heat conduction.

This equation is presented in chapter 4 as equation 4.2 . 